\title{
$\frac{327}{11.17-78}$ S DR, 742 \\ DOE/NASA CONTRACTOR REPORT

DOE/NASA CR-150788

\section{DESIGN PACKAGE FOR CONCENTRATING SOLAR COLLECTOR PANELS}

Prepared from documents furnished by

Northrup, Inc.

302 Nichols Drive

Hutchins, Texas 75141

Under Contract NAS8-32251 with

National Aeronautics and Space Administration

George C. Marshall Space Flight Center, Alabama 35812

For the U. S. Department of Energy

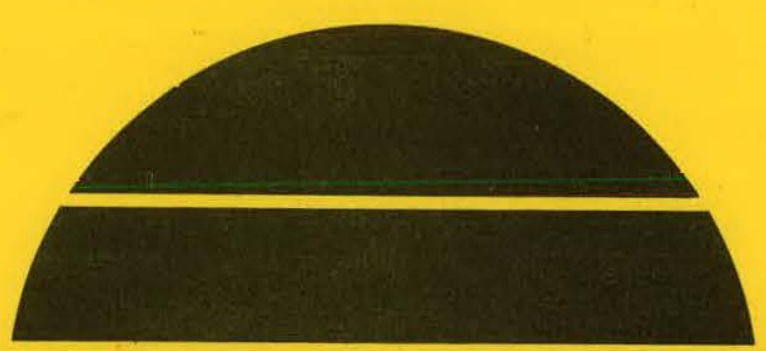

\section{U.S. Department of Energy}




\section{DISCLAIMER}

This report was prepared as an account of work sponsored by an agency of the United States Government. Neither the United States Government nor any agency Thereof, nor any of their employees, makes any warranty, express or implied, or assumes any legal liability or responsibility for the accuracy, completeness, or usefulness of any information, apparatus, product, or process disclosed, or represents that its use would not infringe privately owned rights. Reference herein to any specific commercial product, process, or service by trade name, trademark, manufacturer, or otherwise does not necessarily constitute or imply its endorsement, recommendation, or favoring by the United States Government or any agency thereof. The views and opinions of authors expressed herein do not necessarily state or reflect those of the United States Government or any agency thereof. 


\section{DISCLAIMER}

Portions of this document may be illegible in electronic image products. Images are produced from the best available original document. 
This report was prepared to document work sponsored by the United States Government. NeIther the Unfted States nor 1ts agents the United States Department of Energy, the United States National Aeronaut 1 cs and Space Administration, nor any federal employees, nor any of their contractors, subcontractors or the1r employees, make any warranty, express or implied, or assume any legal liab1lity or responsibility for the accuracy, completeness, or usefulness of any information, apparatus, product or process disclosed, or represent that its use would not Infringe privately owned rights. 
TECHNICAL REPORT ST ANDARD TITLE PAGE'

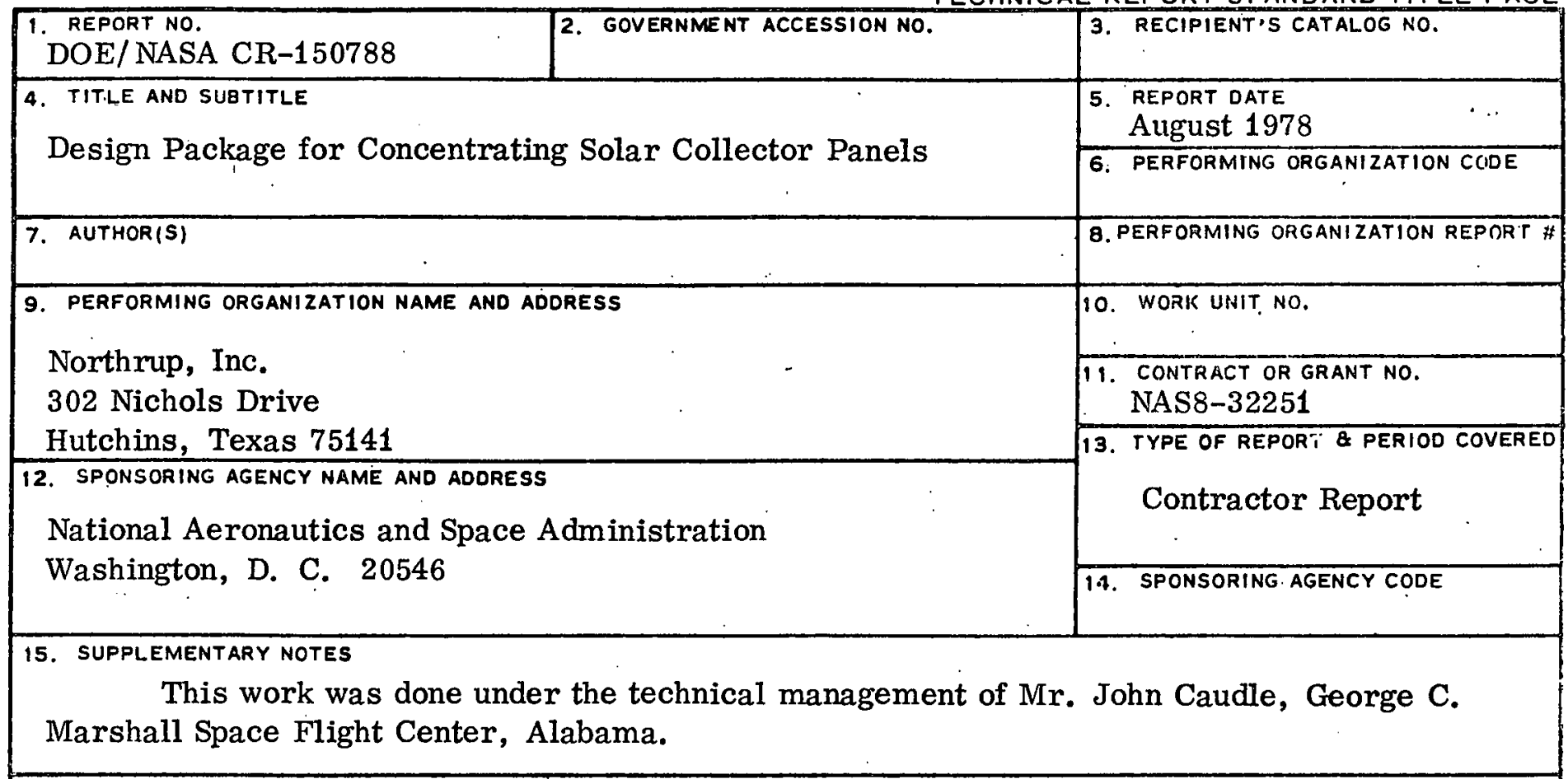

18. ABSTRACT

This report contains information used to evaluate the design of the Northrup concentrating collector. Included are the System Performance Specifications, the applications manual, and the detailed design drawings of the collector.

The Northrup concentrating colar collector is a water/glycol/working fluid type, dipped galvanized steel housing,transparent acrylic Fresnel lens cover, copper absorber tube, fiber glass insulation and weighs 98 pounds. The gross collector area is about $29.4^{2}$ per collector. A collector assembly includes four collector units within a tracking mount array.

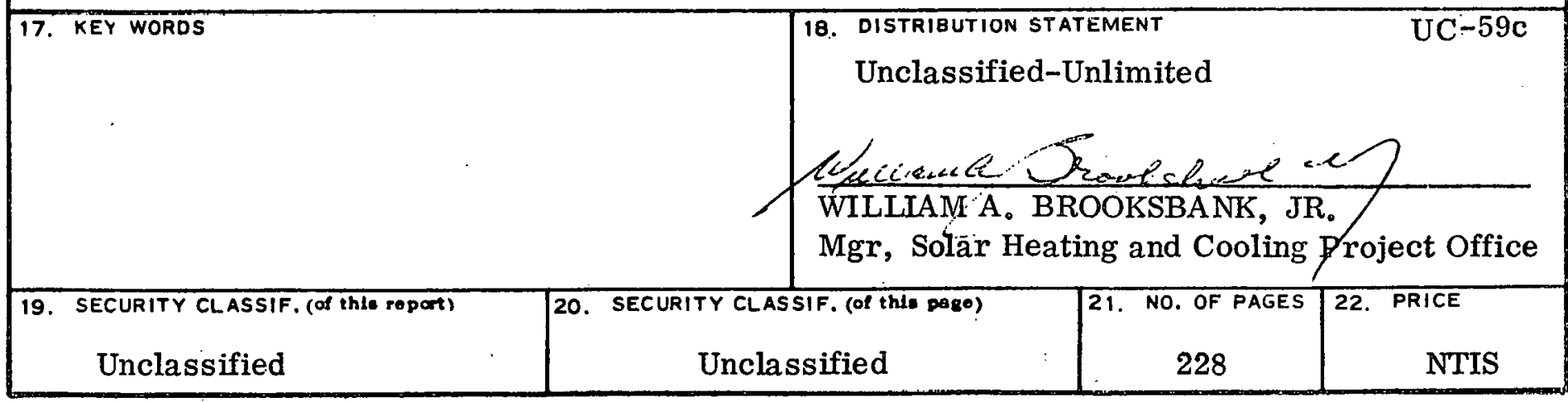


THIS PAGE

WAS INTENTIONALLY

LEFT BLANK 


\section{TABLE OF CONTENTS}

Application Manual

General Description $\quad: 2$

Application $\quad 3$

Model Numbers $\quad$ · $\quad 4$

$\begin{array}{ll}\text { Manifold Sizes } & 4\end{array}$

Flow Rate $\quad 4$

Freeze Protection . 4

Piping \& Fluid System $\quad 5$

System Temperature Control 5

Loads \& Reactions $\quad 7$

$\begin{array}{ll}\text { Subsystem Performance Specification } & 13\end{array}$

Drawing List

WOFICE MN ONLY.

MORTIOHS OF THIS REPORT ARE ILLEGIBLE. IS

has be6n ronpoduced from the best available copy to permit the broadest possible avails ablility.

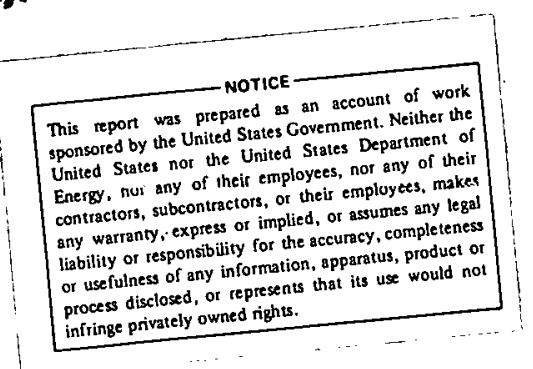


01-06-122 $\because$ Wrapper for Tracking Control Box 61

01-06-002 Electrical Comp. Board 62

01-06-003 Electrical Component Board Lip $\quad 63$

01-18-010 Power Screw \& Nut Trk Cont'1 Box $\quad 64$

01-07-023/24 Housing-Power Screw, Trk Contl Box 65

01-18-014 Cable Guide 66

01-06-133 Cover for Tracking Control Box 67

01-06-123/124 End Panel-N\&S Trk Ctr'1 Box '. 68

$01-06-125 \quad: \quad$ Bracket-Bearing,Trk'g Cont'r Box $\quad: \quad \therefore \quad: \quad: 69$

$\begin{array}{lll}01-17-118 & \text { Control Box Bearings } & 70\end{array}$

01-06-136 Motor Mtg Bracket for Tracking Motor 71

01-06-126/127 Cover-Limit SW Trk Control Box 72

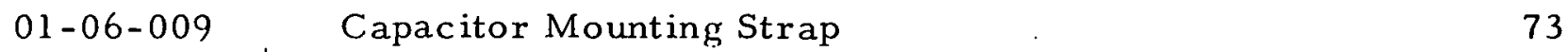

$\begin{array}{lll}01-17-120 & \text { Control Box Drive Sprocket } & 74\end{array}$

01-17-119 Drive Motor Sprocket . . 75

01-17-121 Control Box Drive Chain 76

01-10-004 Transformer Tracking Mechanism 77

01-10-008 Relay Socket Tracking Mechanism $\quad 78$

01-02-002 Shop Subassembly Electrical Component Board _. . 80

01-10-002 Tracking System P/C Board Assembly 81

01-10-009 PC Board Connector Tracking Mechanism 85 
$01-02-027$

Pulley 29" Dia. Shop Sub-As sembly

$01-06-132$

Pulley Cable Clamp Plate

$01-07-30$

Pulley Mount Angle

01-07-025

Pulley Rim

$01-07-026 / 27 /$ 088

Pulley Spoke

01-07-029

Pulley Hub

01-03-051

Lens End Closure Assembly

$01-06-112$

Lens End Closure Ënd Plate

100

$01-06-137$

Lens End Closure Weather Strip Plate

101

$01-20-001$

Collector Lens

102

$01-06-030$

Lens Retainer Top

104

$01-03-049$

Collector Housing Assembly

105

01-06-109

Insulation Cap Fastener

106

$01-06-051$

End Connector Trussed Torque Bar

107

$01-02-019 / 20$

Collector Truss Sub-Assembly

108

$01-06-029$

Lens Guide Col'tr Panel

$01-06-138$

Lens Guide Splice Collector Panel

110

$01-06-050$

Truss Connector Torque Bar-Bot.

$01-06-049$

Truss Connector Torque Bar-Top

112

01-02-012

Absorber Tube Sub-Assembly

113

01406-111

Absorber Tube Retainer

114 


\begin{tabular}{|c|c|c|}
\hline $01-08-023-27$ & Absorber Tube(s) Crossover and Riser & 115 \\
\hline $01-08-020 / 21$ & Absorber Tube Tubes \& Cap & 116 \\
\hline $01-06-108$ & High Limit Switch U-Fastener & 117 \\
\hline $01-10-019$ & High Limit Switch Bridge Fastener & 118 \\
\hline $01-06-048$ & Torque Bar Bottom & 119 \\
\hline $01-06-064 / 79$ & Bridging Connector to Torque Bar Collector Panel & 120 \\
\hline $01-02-009$ & Torque Tube Sub-Assembly & 121 \\
\hline $01-06-024$ & Web Member-Wide Collector Truss & 122 \\
\hline $01-06-025$ & Web Member-Narrow Collector Truss & 123 \\
\hline $01-06-026$ & Chord-Top \& Bottom Collector Truss & 124 \\
\hline $01-06-063$ & Bridging Vertical Collector Panel & 125 \\
\hline $01-06-027$ & End Strut Collector Truss & 126 \\
\hline $01-06-028$ & End Beam Collector Truss & 127 \\
\hline $01-02-007$ & End Beam Sub-Assembly & 128 \\
\hline $01-06-061$ & Bridging-Cross Collector Panel & 129 \\
\hline $01-06-062$ & Briding Top \& Bot. Collector Panel & 130 \\
\hline $01-06-040$ & Cover Sides \& Bot Collector Panel & 131 \\
\hline $01-18-005 / 6$ & Shaft-N\&S End Collector Panel & 132 \\
\hline $01-02-010 / 11$ & Flanged Shaft Assy N\&S End Collector Panel & 133 \\
\hline $01-07-017$ & Flange - Shaft Collector Panel & 134 \\
\hline $01-17-098$ & Wind Brace Lower & 136 \\
\hline $01-17-100$ & North and South (Upper) End Wind Brace & 137 \\
\hline \multirow[t]{2}{*}{$01-17-099$} & North (Lower) Upper Wind Bracing & 138 \\
\hline & vi & \\
\hline
\end{tabular}




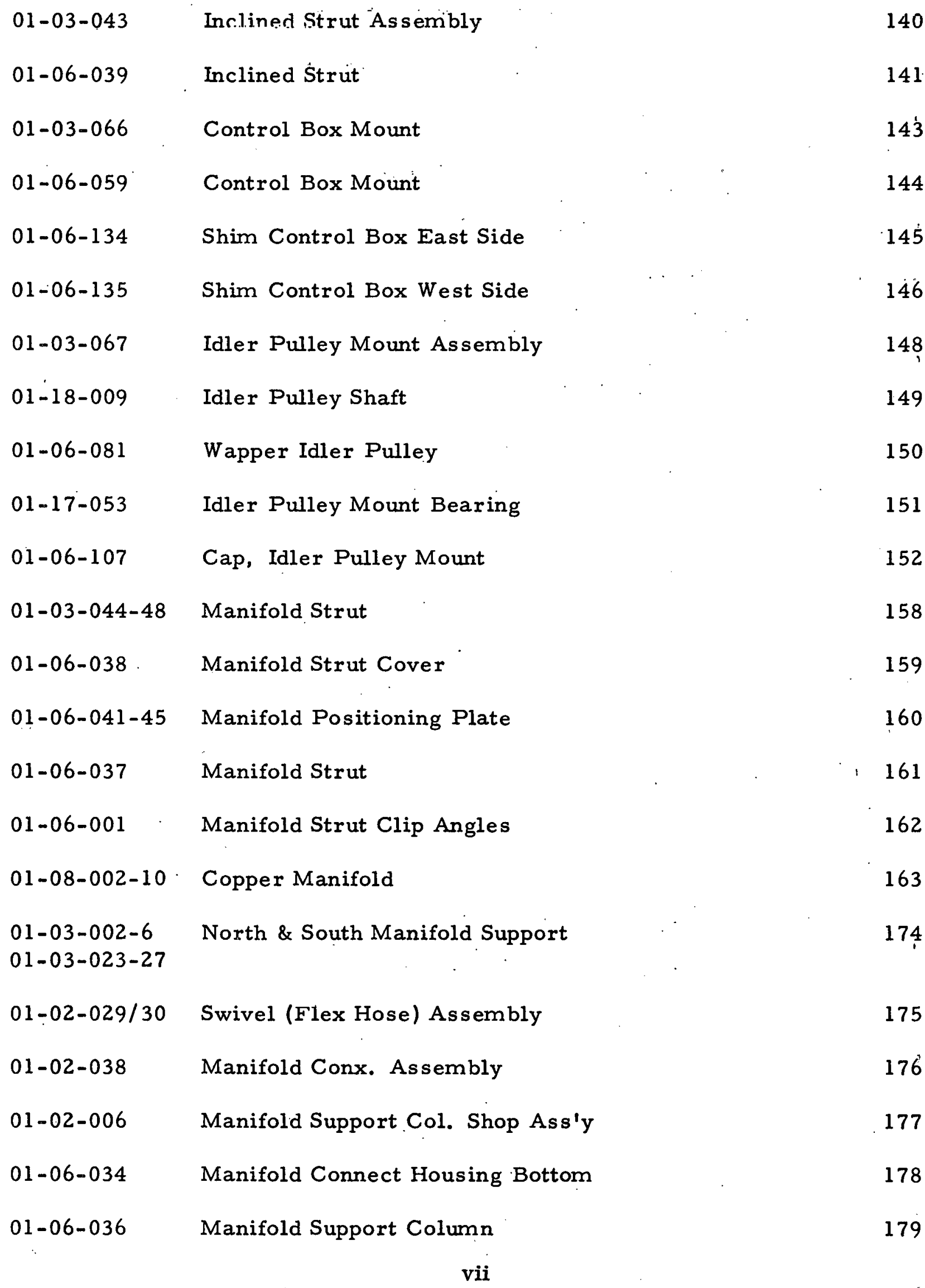




$\begin{array}{llr}01-06-053 & \text { Closure Plate Blank } & 211 \\ 01-03-082 & \text { Cable Guide Bracket Assembly } & 212 \\ 01-06-173 & \text { Bracket Cable Guide } & 213 \\ 01-03-053 & \text { Solar Sensor Sub-Assembly } & 215 \\ 01-06-110 & \text { Solar Sensor Mount } & 216 \\ 03-06-104 & \text { Sensor Positioning Bracket } & 217 \\ 01-20-004 & \text { Sensor Tube } & 218 \\ 01-10-015 & \text { Solar Discs Base } & 219 \\ 00-10-011 & \text { Solar Discs for TRD-3 } & 220 \\ 01-10-039 & \text { Solar Sensor Discs } & 221 \\ 01-20-002 & \text { Solar Sensor End Cap } & 222\end{array}$


APPLICATION MANUAL

\author{
FOR \\ NORTHRUP CONCENTRATING SOLAR COLLECTOR PANELS \\ MF-NSC-P "ML" SERIES \\ MODEL NSC-01-0732
}


GENERAL DESCRIPTION: "ML Series" Solar Collector Panels comprise a complete packaged array consisting of collector panels, supporting framework, insulated fluid manifolding and tracking drive system, shipped from the factory in unassembled components for field erection. Each collector panel has four lenses, each having a net aperture of $10.75 \mathrm{ft}^{2}$ making a total of $43 \mathrm{ft}^{2}$ for each collector panel. Overall size of each collector panel is $583 / 8 " \times 1335 / 8$ ". Panels are spaced 10'-0" on center. Various components are described as follows:

Lens: Modified, Fresnel, prismatic lens extruded from acrylic. Transmissivity of acrylic is 0.95 . Lenses have an 8 " outside radius and short focal length with precision manufacturing for high efficiency. Concentration is 10 to 1 .

Absorber Tube: Made of copper, formed to an eliptical shape for maximum collection for all seasons. Absorber tubes have selective coating having an absorbtivity of 0.95 and an emissivity of 0.12 .

Collector Panel Housing: Constructed of galvanized steel, consisting of light trusses, heavy torque bar, and heavy end beams, all designed to provide a rigid panel. This framework is covered with light gauge galvanized steel to form the complete housing.

Insulation: The collector panel is insulated with fiberglass having a "k" factor of 0.28 at $200^{\circ} \mathrm{F}$. Thickness of this insulation is $3^{\prime \prime}$ in bottom, 1 13/16" in ends and sides and 1 " at each side of absorber tube, extending up 8".

Framing Supports: Each collector panel is supported by a double "A" frame, as shown on DWG. 01-05-122, constructed of heavy gauge galvanized steel to withstaild loads as indicated on Figures 2 and 3 . Frames and collector panels are spaced 10'-0" 0.C. The frames are connected together with struts and braced by " $X$ " wind bracing. All fastening devices are zinc plated and are furnished complete for the entire array. Dead load, live load and wind load reactions, resulting from design loads are shown in Figures 2 and 3.

Anchor bolts are not furnished, since structures on which arrays are mounted vary with each installation. Anchor bolts must be designed for loads given. REF DWG. 01-05-123

Manifolds: All fluid manifolds are type "M" copper and connect each collector panel together. They are contained in the manifold strut which acts as a structural member as well as a weather tight housing to protect the insulation. These manifolds are completely assembled and insulated with 1 1/2" to 2 " of fiberglass having a "k" factor 0.30 at $200^{\circ} \mathrm{F}$. 
Expansion Compensation: Expansion in the manifold is compensated by flexible swivel connection in each manifold support to allow all differential expansion between copper lines and steel housing to be distributed to East and West ends of the array. Flexible expansion compensator as depicted in Figure 1 are field supplies and installed to compensate for this movement with system piping.

Tracking system: System is designed for east-west tracking, with axis of collectors mounted parallel to polar axis. Collector panels are linked together with a system of heavy, galvanized aircraft cable and p̈ulleys. This system is driven by a mechanism consisting of a two directional motor, chain drive, stainless steel (Acme) screw and a "Delvin" drive nut which does not require lubrication. The two directional motor is controlled by photovoltaic cells through an integrated circuit to constantly maintain collector panels in focus on the sun. In this circuit is a low light level which directs the collector to track to East during night time, and stop at the proper position for sunrise. Tracking angle is set for four hours before and four hours after solar noon which is the optimum for non-shading between collector panels.

APPLICATION: These series of collectors are applicable to any. system requiring fluid temperatures up to $240^{\circ}$. Water or a combination of. water and ethylene glycol. is recommended as the heat transfer fluid. (See Paragraph on Freeze Protection) Other solutions may be used, provided they are compatible with copper, ethylene propylene rubber, neoprene rubber and silicone rubber which are used in components of fluid system in the collector arrays.

These arrays are installed with the long axis of the lenses pointing to true north and tilted to face south at an angle to horizon (tilt angle) equal to the north latitude. In this manner the rotation. for east-west tracking is always parallel to the polar axis of the earth.

Arrays are available with a minimum of two collector panels and a maximum of eleven collector panels in one array. When more than eleven panels are required (473 $\mathrm{ft}^{2}$ net aperture), additional arrays are used, and installed with the same 10'-0" spacing between panels, but without connecting manifolds between arrays.

Standard models are avialable with tilt angles ranging from $10^{\circ}$ to $55^{\circ}$ in $5^{\circ}$ increments. Other tilt angles. between these are available on special order and a slight increase in price. 
MODEL NUMBERS: Arrays are ordered by model numbers and coding is explained as follows:

Northrup Solar Collector

"MU": Series

$$
\text { NSC }-01-0000
$$

Tilt Angle as indicated by last two digits of Model No.

Number of Collector panels in the Array

EXAMPLE: Model No. NSC-01-0732

\author{
"ML" Series \\ Seven Collector Panels \\ $32^{\circ}$ Tilt Angle \\ $7 \times 43=301$ Net. Sq. Ft. in the array
}

MANIFOLD SIZES: Manifold sizes are arranged in a reverse return, with inlet to array at the southwest end and outlet at the northeast end. Sizes are as follows for south manifold starting from the east and are in reverse order for north manifold:

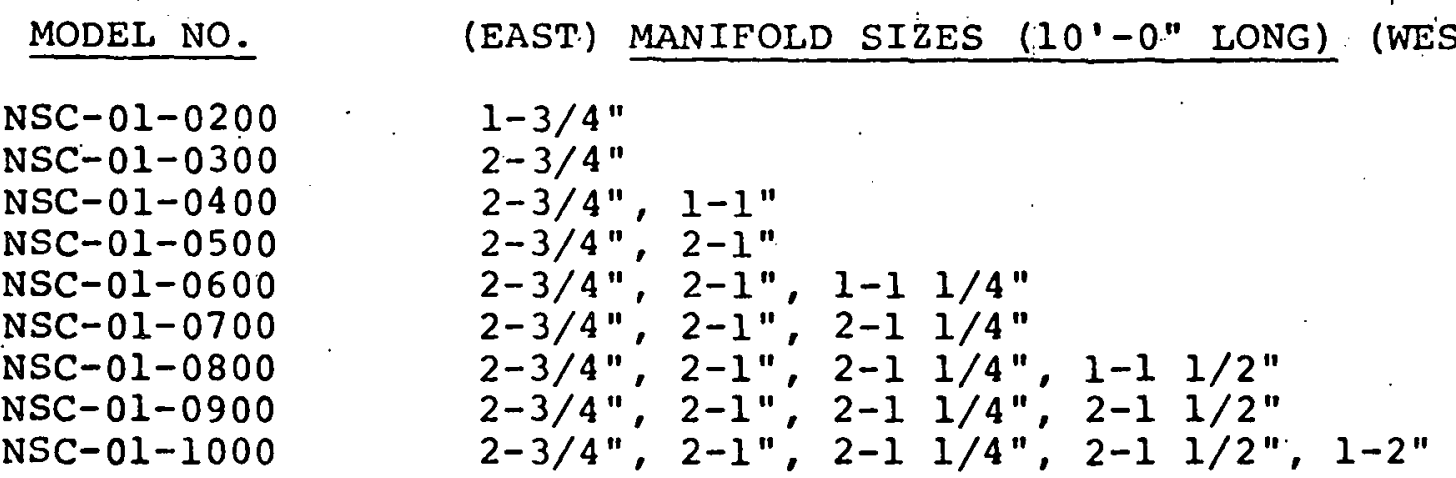

FLOW RATE: Recommended flow rate through each collector panel is 1.72 to 1.3 GPM. Figure 5 is actual pressure drop test through one collector panel at various flows. Pressure drop through manifolds can be determined by ASRAE standards. Manifold sizes listed above are selected to produce a pressure drop of not more than $3^{\prime}$ head of water for $100^{\circ}-0^{\prime \prime}$ equivalent feet of pipe a 1.72 GPM per collector panel. Where manifolds change size every 10'-0", a reducing tee connects these pipes, with a $1 / 2$ " connection to flexible swivel of the collector panel.

FREEZE PROTECTION: This series of collectors are such that when system is drained, a small amount of fluid will be retained in the south absorber tube risers and in the south flexible swivel

connections. Therefore a complete drain-down anti-freeze system is not applicable. In areas of infrequent days of freezing, warm water of the system may be circulated by means of an automatic low temperature sensor controlling pump of system during non-solar hours and/or below freezing periods. 
In areas of frequent freezing periods an anti-freeze solution is required. Ethylene glycol is recommended for protection at various temperatures as follows:

$\begin{array}{rr}\text { TEMP } & \circ \mathbf{F} \\ 16 & 8 \text { ETHYLEN } \\ 10 & 20 \\ 0 & 25 \\ -12 & 33 \\ -34 & 40 \\ -62 & 50 \\ & 60\end{array}$

Each collector panel has a total volume of 3 gallons: Volume of each 10'-0" copper manifold is as follows:

\begin{tabular}{cr} 
MANIFOLD SIZE & GALS PER $10^{\prime}$ \\
\cline { 2 - 2 } $3 / 4^{\prime \prime}$ & 0.27 \\
$1 "$ & 0.46 \\
$11 / 4^{\prime \prime}$ & 0.68 \\
$11 / 2^{n}$ & 0.95 \\
$2^{n}$ & 1.65
\end{tabular}

PIPING \& FLUID SYSTEM: The following recommendations are made in the interest of providing viable system compatible with these systems.

Filtration: A strainer or filter is recommended ahead of the pump of the system to maintain system free of debris. This should be maintained frequently at start-up of system.

Valves and Controls: Figure 1 represents optimum of control valves and sensing devices. These arrays are designed for an operating pressure of 100 PSI at $240^{\circ} \mathrm{F}$, with mechanical pressure relief at 125 PSI. Shut-off and balancing valves provide means to control the flow and to isolated array during repair. Temperature and pressure sensing wells provide means of balancing flow rate when more than one array is used. Drain valve provides a convenient method to make repairs to the array without affecting other system piping. An optional high limit control is available to defocus the collectors should temperature limit be exceeded.

SYSTEM TEMPERATURE CONTROL: Since function or application varies with each installation, temperature controls must be individually designed. It is recommended the manufacturer be consulted for recommendations regarding these controls. 

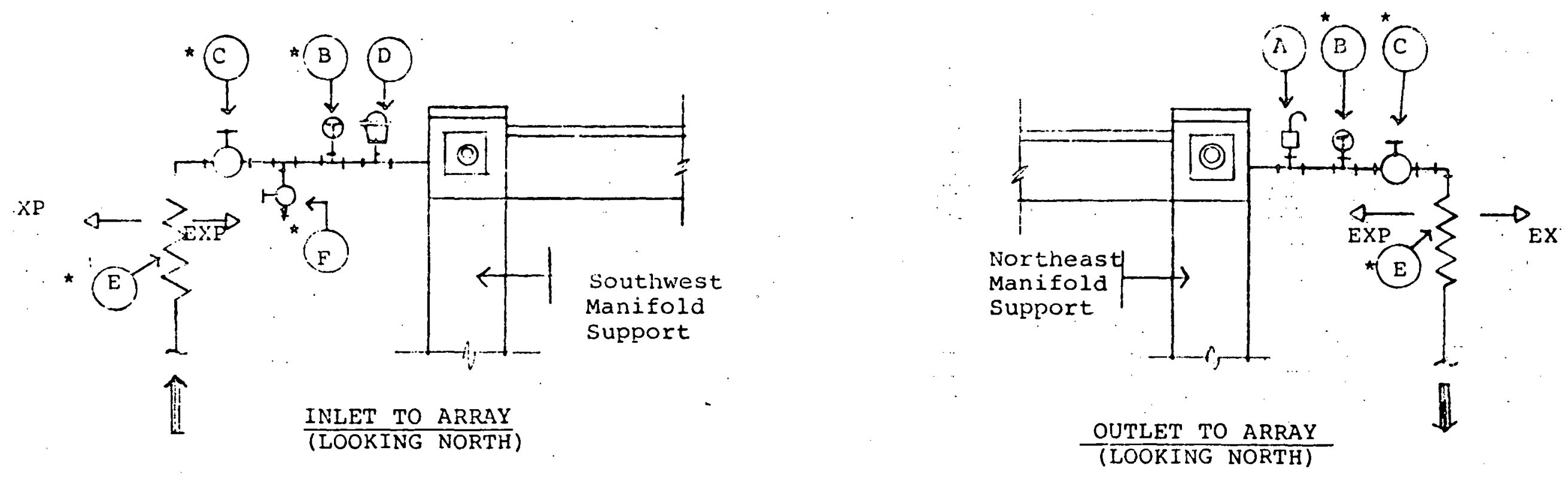

\section{SYMBOLS}

(A) Automatic air eliminator, furnished by manufacturer:

* B) Combination pressure and temperature measurement device. "Petes Plug" or equal.

*(C) Combination balancing and shut-off valve. Ball valve recommended.

(D) Pressure relief valve, furnished by manufacturer.

* E Flexible connection to provide East-West expansion of array piping, $\pm 0.1^{\prime \prime}$ per 10'-0" of manifold piping.

* (F) Drain cook with hose dibb connection.

* Not supplied by manufacturer

RECOMMENDED PIP $\$ N G$ ACCESSORIES TO COLLECTOR PANEL-ARRAY 


\section{DESIGN LOADS}

D. L. = Actual operating dead weight of structure including water as the flivid.

L. L. = One inch of ice on all surfaces of each member, 4.67 1bs/S.F. of member surface.

W. L. $=70 \mathrm{mph}$ wind, at $30^{\circ}$ above ground surface.

REACTIONS (1bs, at every 10'-0" O. C. in East-West direction)

SOUTH

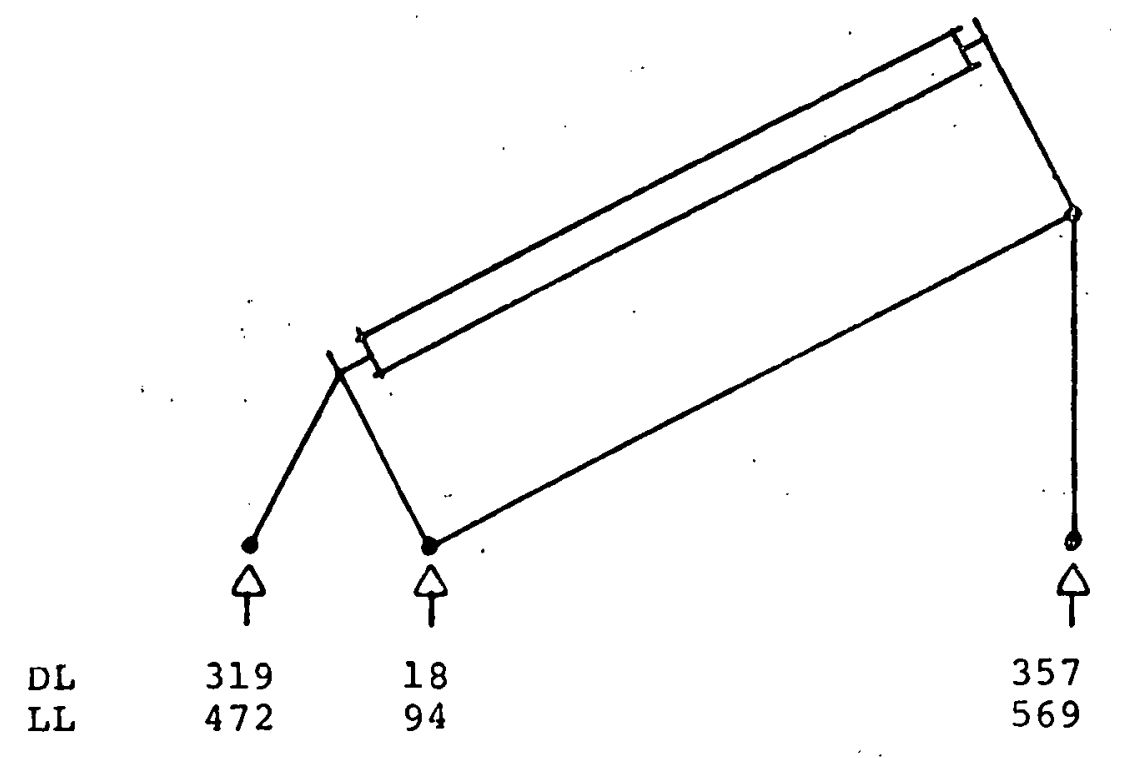

NORTH

MODEL NSC-01-0732 


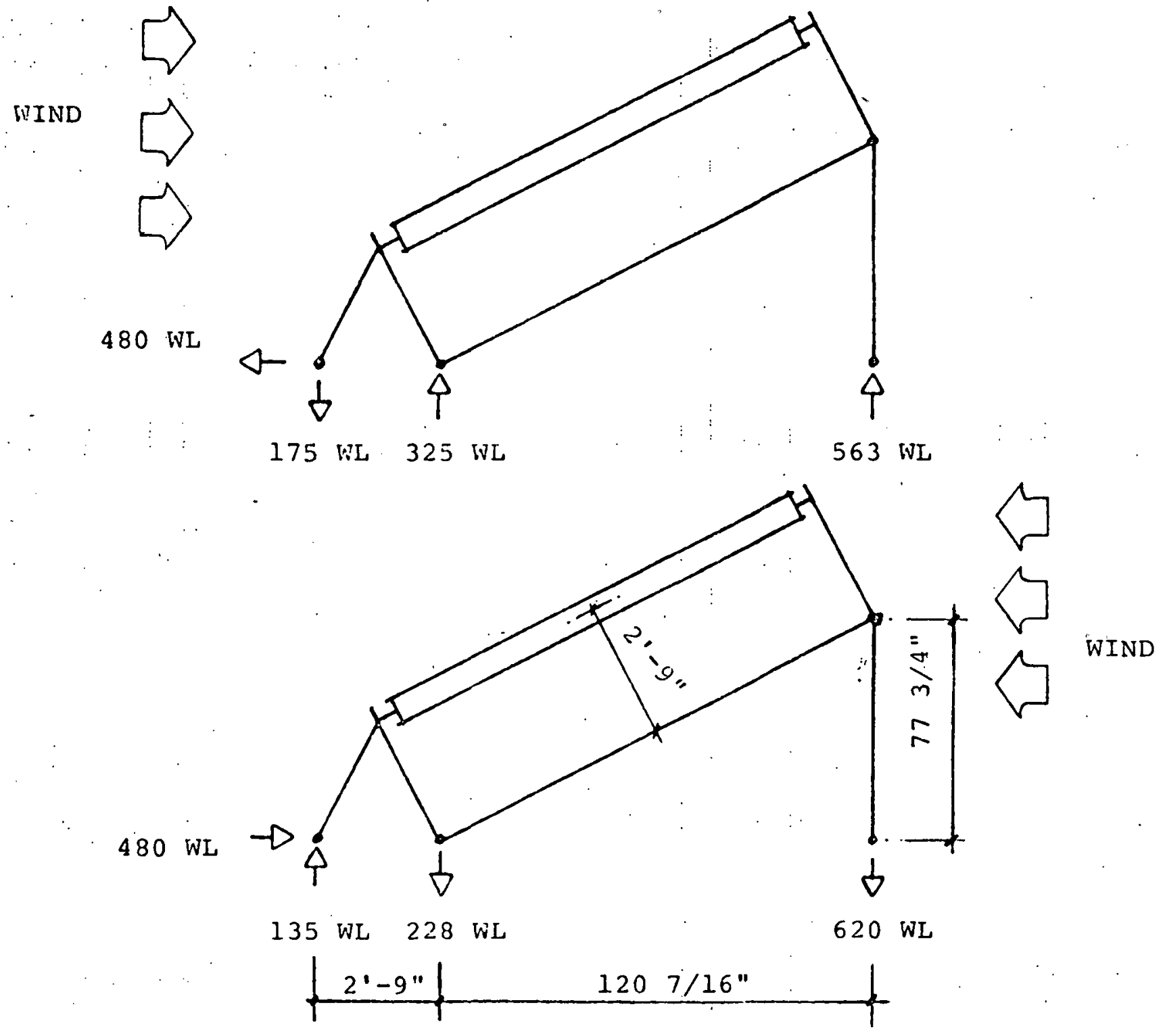

MODEL NSC-01-0732

Fig. 3 


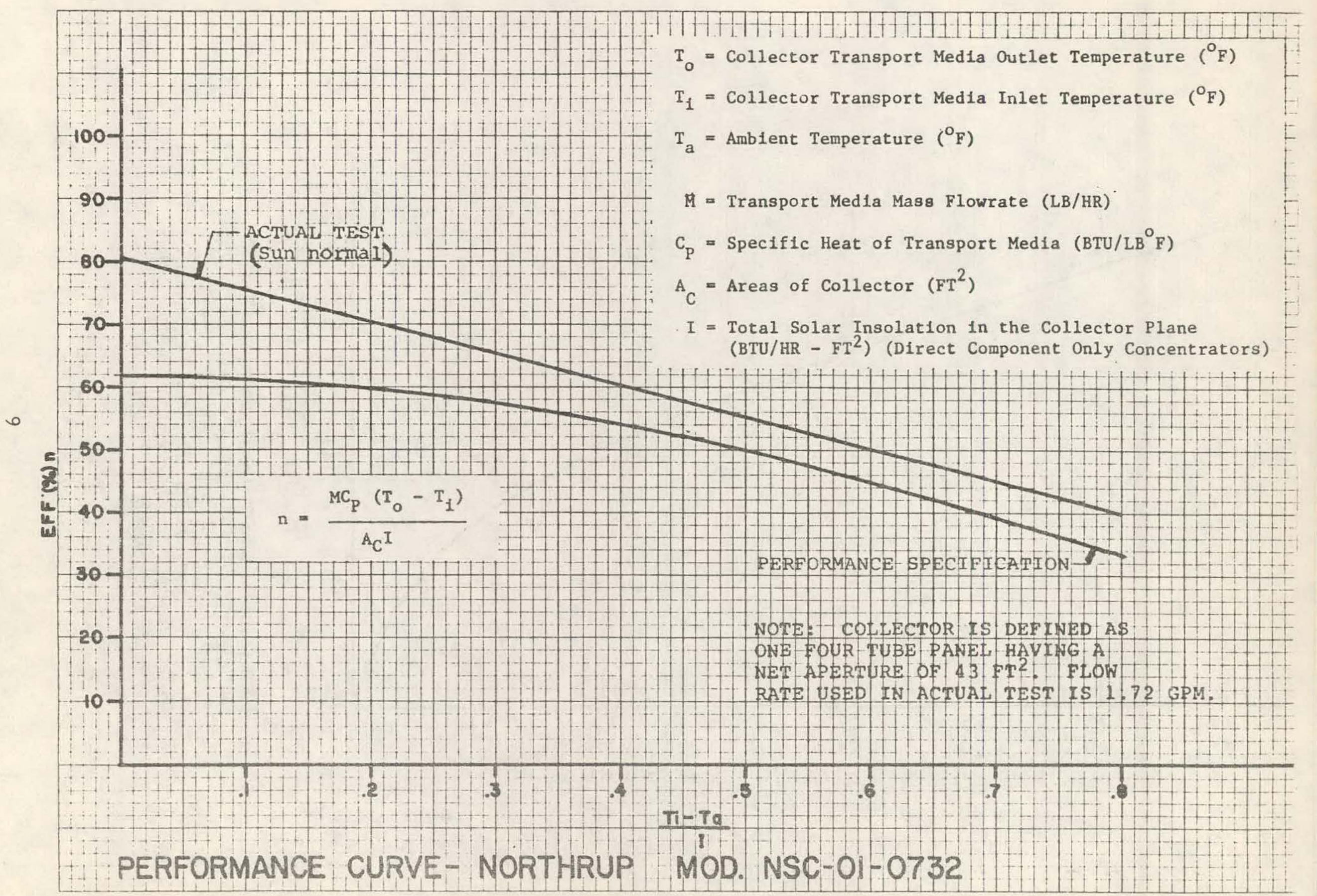




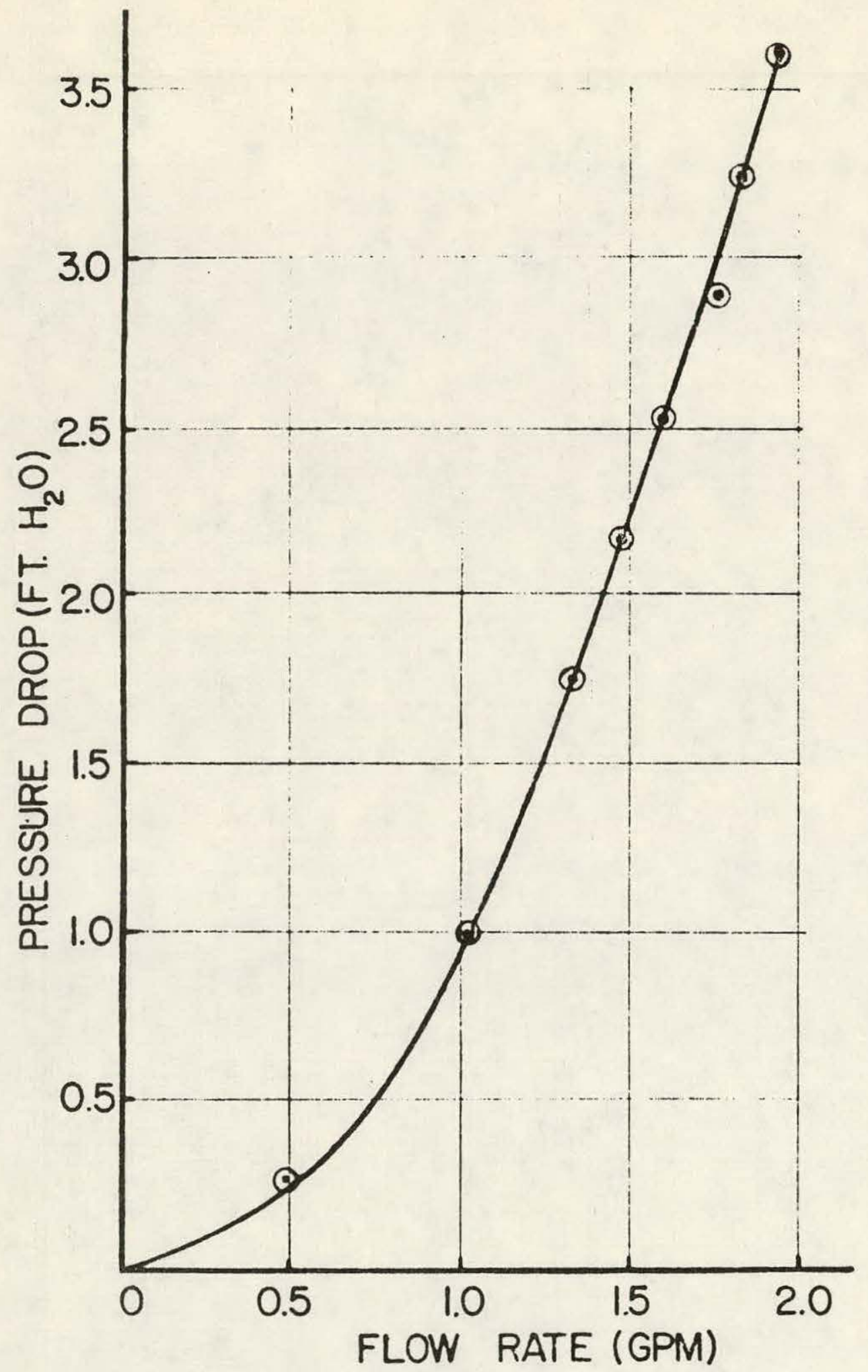

PRESSLRE DROP THRL OME, HORTHRUP CONGENTRATING SOLAR COLLECTOR PANEL ( 43 FT.2 NET APERTURE)

ME-NSC-P-ML SERIES (ONE PANEL CONSISTING OF 4 LENO AND 4 ABSOREER TUBES)

$$
\text { FIGURE 5 }
$$



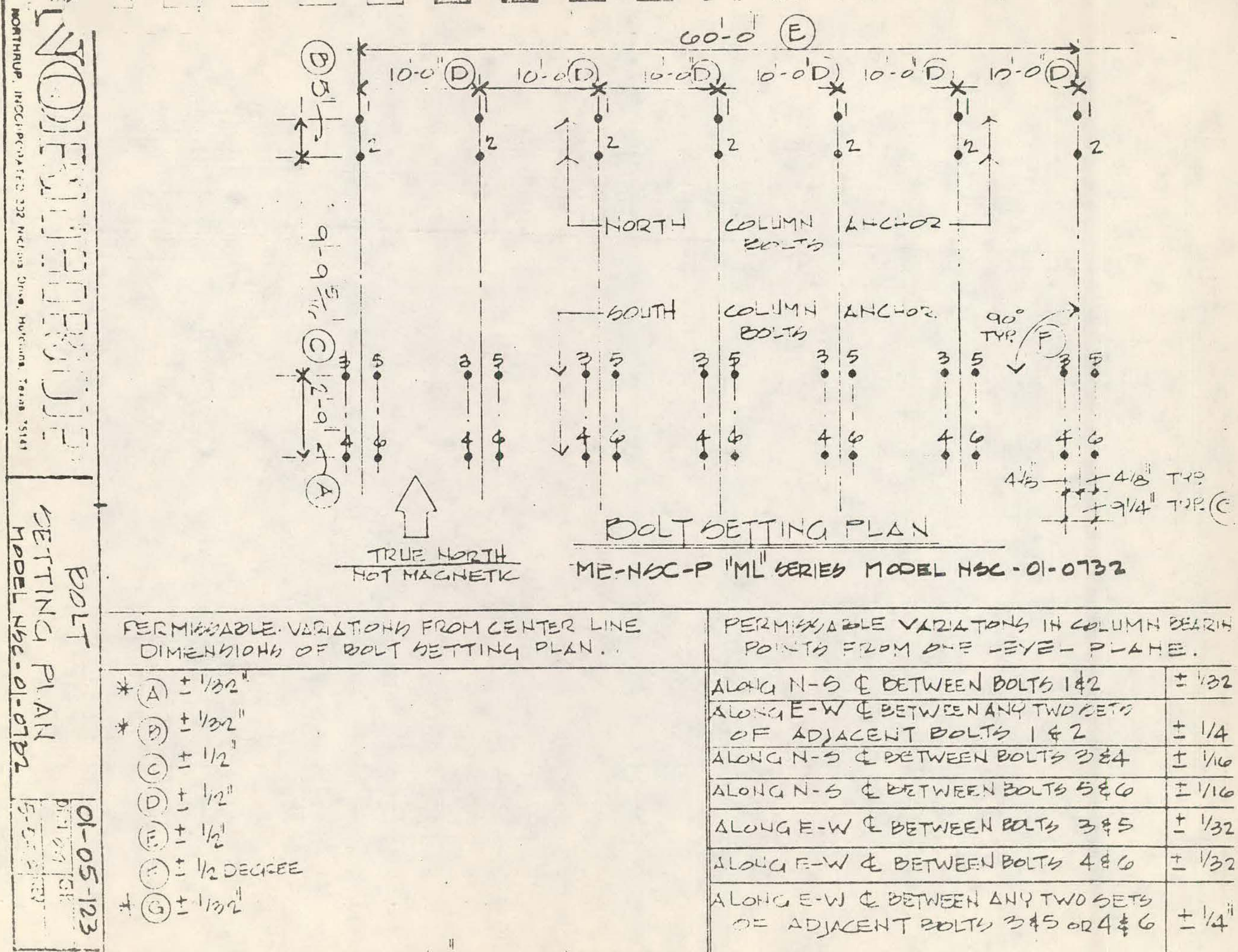

TRUE HORTH MOT MANETK ME-NSC-P I'ML' SERIES MODEL NSC-01.0732

FERMMHAOLE. VIZITTOH' FROM CENTER LINE DIMIENhIOHh OF BOLT hETTING DLAN.

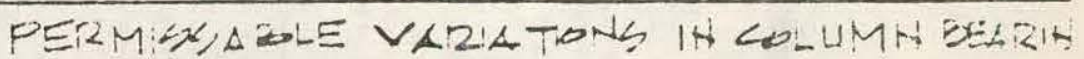
Pol-Th F 20:M DUE - EYE- D-AHE.

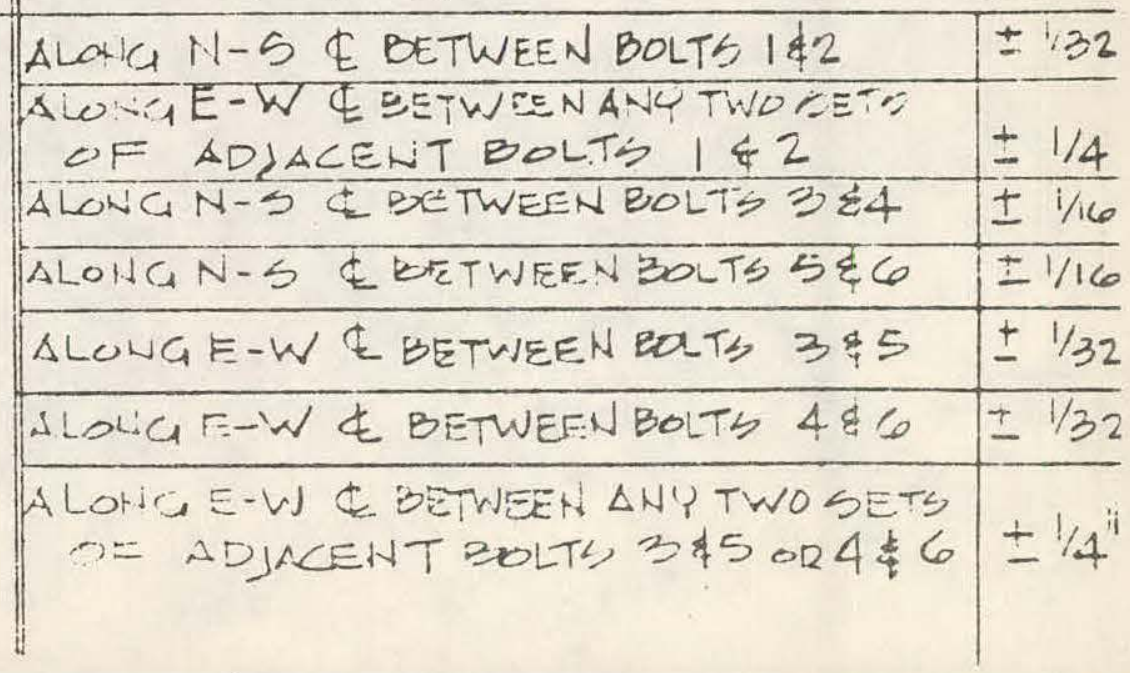




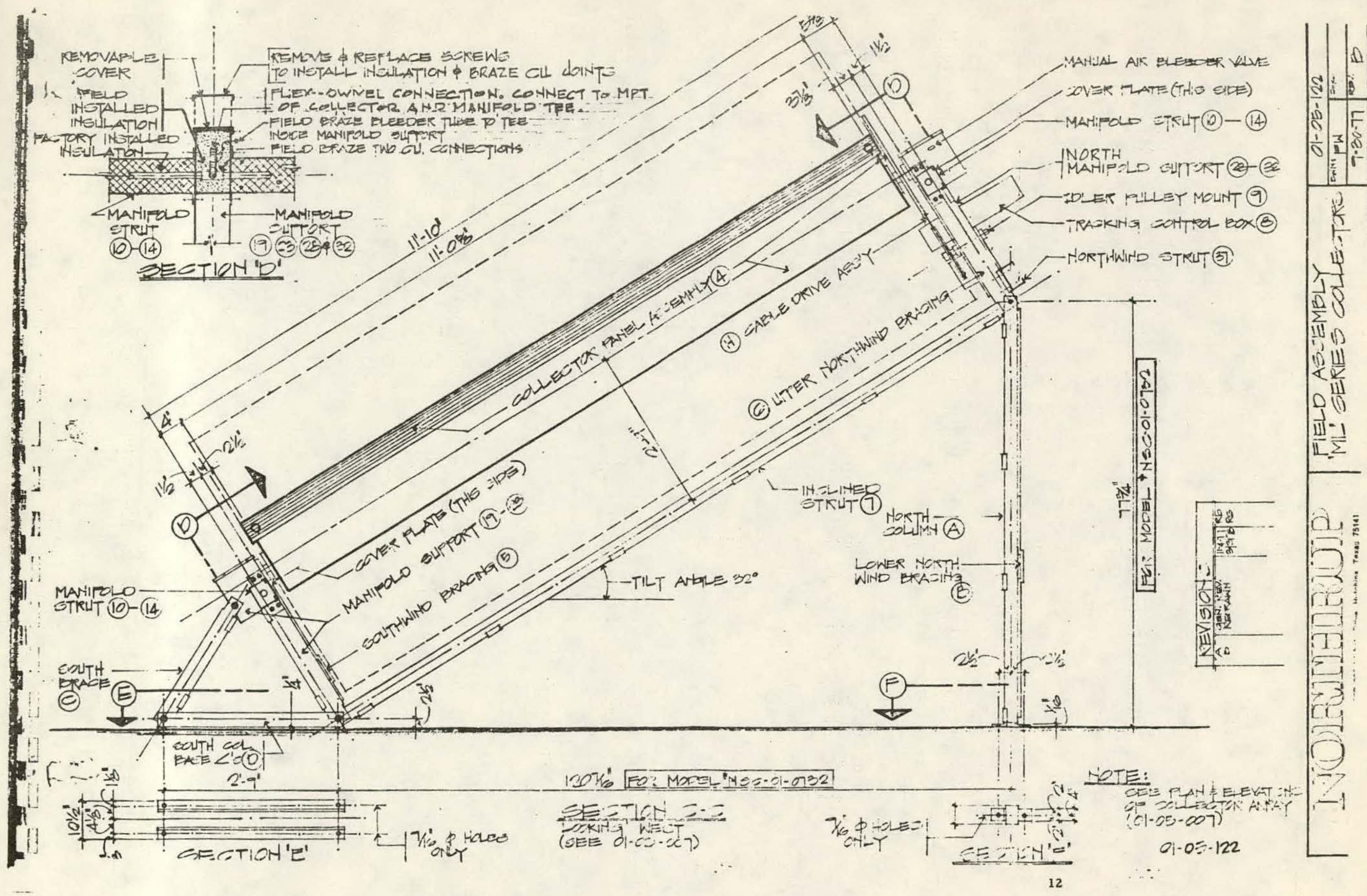


SUBSYSTEM PERFORMANCE SPECIFICATION

Nor thrup, Incorporated

Concentrating Collector

Basic Issue $10 / 1 / 76$ CCBD 301-76-0047

Revision 1 1/20/77

CCBD 301-77-0209

Revision $27 / 14 / 78$

CCBD 301-78-0354 


\section{SUBSYSTEM PERFORMANCE SPECIFICATION}

\section{$1 \because .1$. INTRODUC:TION}

"Thte Performince Spectfication establishes the requirements for the design and perfurmance of the subsystems for use with comblined henting and cooling systems. It designates the Interim Performance Criterla applicable to each type subsystem and defines the deviations. The appendices specify the performance for each subsystem."

\subsection{APPLICABLE DOCIMENT}

\subsection{Government Documents}

Interim Porformance Criterla for Solar Heating and Combined Heat ing Cooling Systems and Dwellings, January 1, 1975. U.S. Department of Housing and Urban Development.

Interlm Performance Criterta for Commerclal Solar Houslng and Comblned Heating/Cooling Systems and Fac1lities; Document No. 98M10001, Reviston Basic, dated February 28, 1975. George C. Marshall Space Flight Center, National Aeronautics and Space Administration.

\subsection{Contractor Documents}

2.2.n. Application Manual for Northrup Concentrating Solar Panels MF-NSC-P "ML" Series Model NSC-01-0732.

2.2.b. Installation Instructions for Northrup Concentrating Collector Panels with Integral Manifold - Frame and Sub-Frames, MF-NSC-P "MF". Series Model NSC-01-0732, Northrup, Inc. 
3.0 APPLICATION OF INTERIM PERFORMANCE CRITERIA BY TYPE OF SUBSYSTEM

"The application of each paragraph of the Interim Performanca Crlterla to each eype subsyatem 1s provided in the following $\operatorname{coble(a)}$

Table 1 - Rasidencial Subayatems

Table II - Comercial Subsystems

4.0 DEVIATIONS FROM INTERIM PERFORMANCE CRITERIA

\subsection{None}

5.0 GOVERMENT FURNISHED PROPERTY

\subsection{None}

6.0 COVERMMENT DIRECTED REQUIREMENTS

No specific requirements have been directed by the Contracting Officer.

\subsection{SUBSYSTEM APPENDICES}

Appendix A Subsystem Identification 
Specification

Revision

Date
SHC -3048

2

$7 \longdiv { 1 4 / 7 8 }$

\subsection{WARRANTY}

Eighteen months from date of shipment under normal usage. Materials and workmanship. 
TAELE I

spgcipication Ho. SHC-3048

aevision

OAPC $\frac{10-1-76}{20}$

RESIOENTIAL SUBSYSTEMS. INTERIM PERFORMANCE CRITER IA SUIMMARY

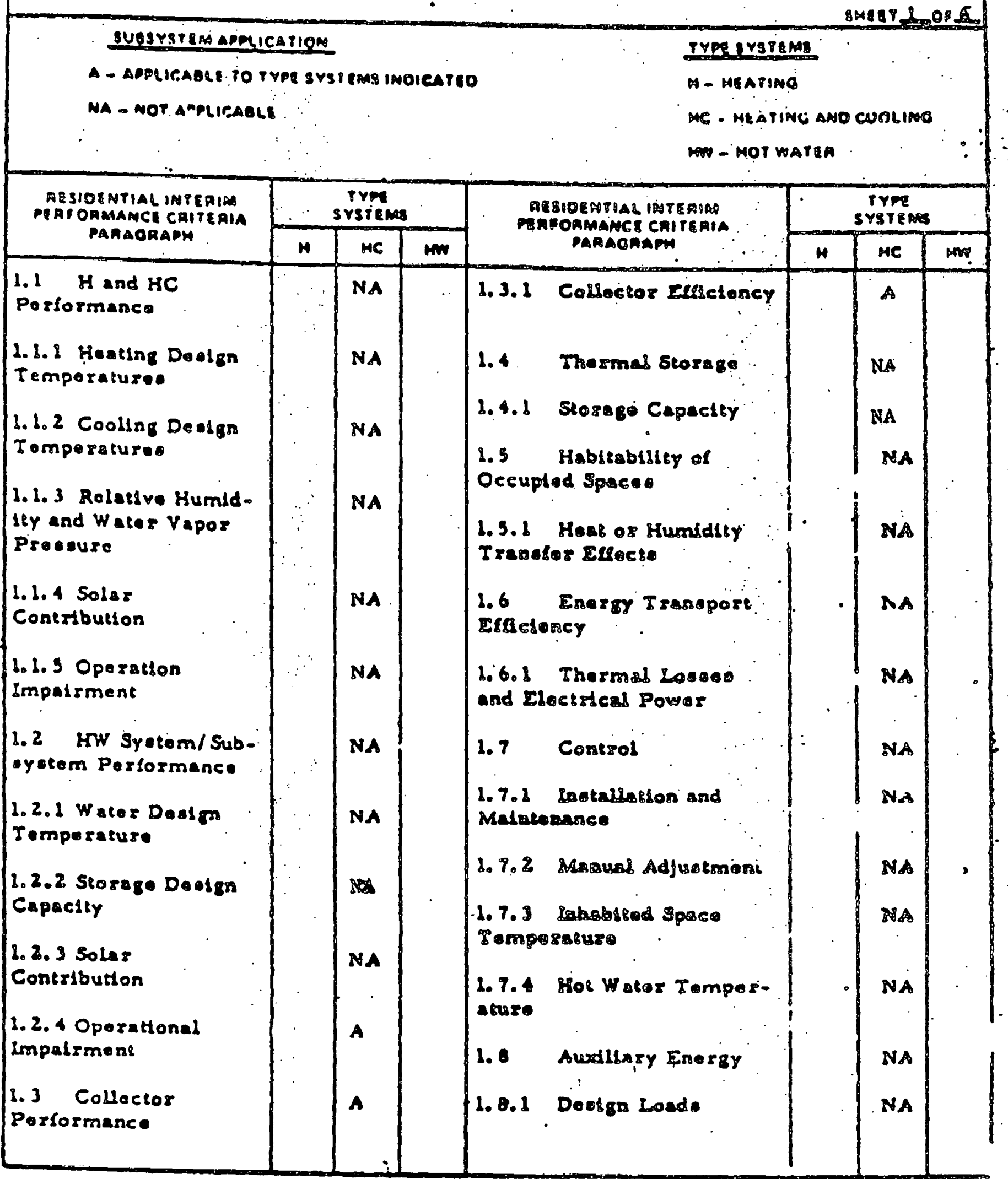


TAALE I

vale $\quad 7 / 14 / 78$

\section{RESIOENTIAI. SU8SYSTEMS, INTERIM PERFORMANCE CRITER IA SUMMARY}

sirli_zon 6

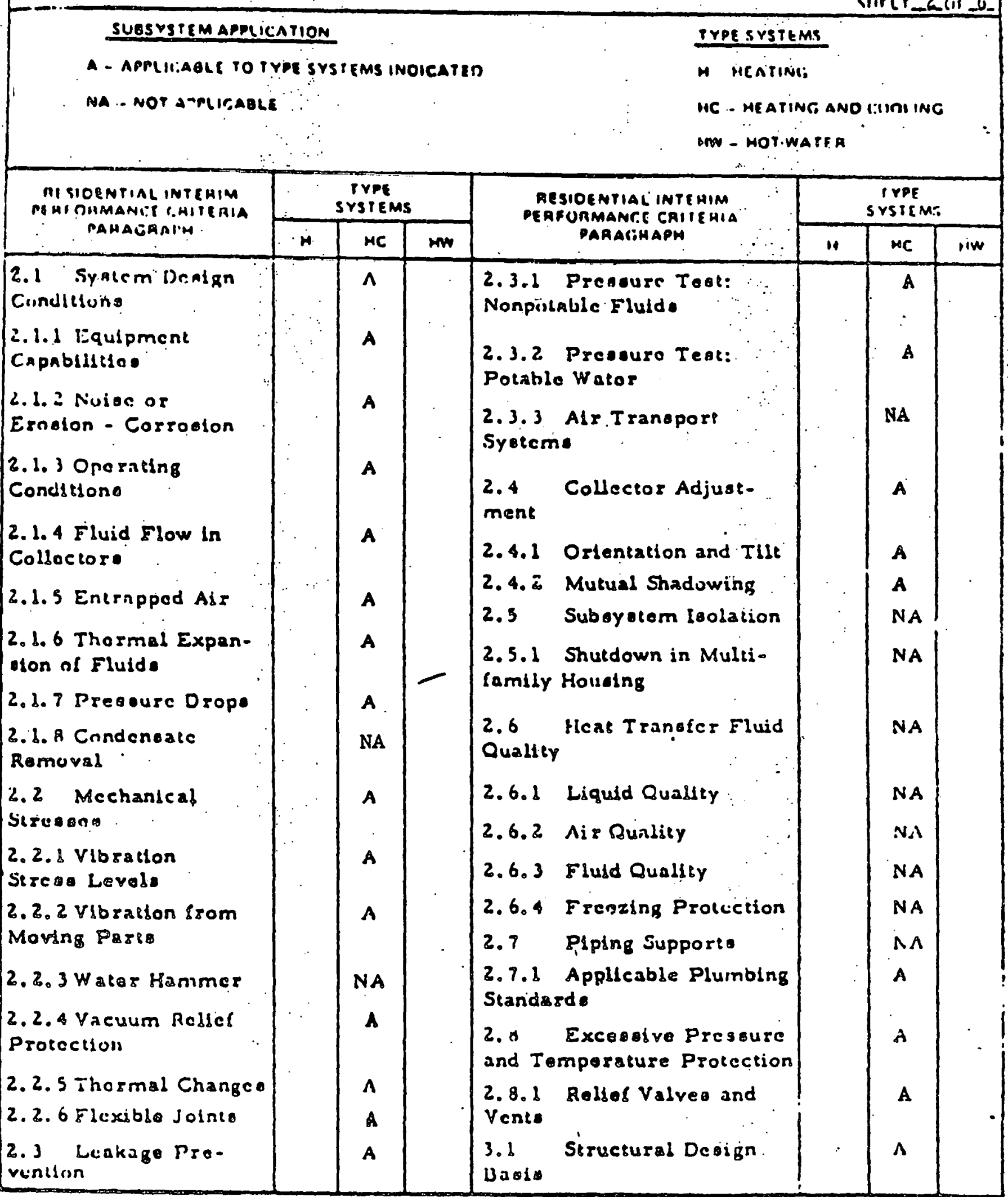


PABL!

irecificaitinn Mn. SHC-3048

ar vision

2.

OAPR $-7 / 14 / 78$

RESIDCNIIAL SURSYSIEMS. INTER IM PERFORMARICE CRITERIA SUMMARY

SUESYSILMAPDLICAIION

a - armlir.agle in type sustima indicaito

na - nOt anrliranLE

nir 6120.6

IYPE IYSTIMS

m meatini:

IIr. III AIINEI ANBS CIIIII INE

MW . MOI WATEA

\begin{tabular}{|c|c|c|c|c|c|c|c|}
\hline \multirow{2}{*}{ 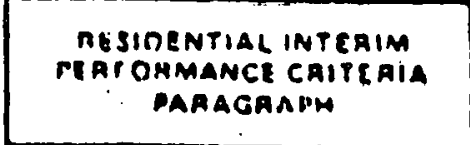 } & \multicolumn{3}{|c|}{$\begin{array}{l}\text { IVPE } \\
\cdots \text { SYYSIEMS }\end{array}$} & \multirow{2}{*}{ 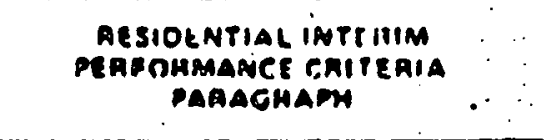 } & \multicolumn{3}{|c|}{$\begin{array}{l}\text { PYPE } \\
\text { SYSTEMS }\end{array}$} \\
\hline & st & MC & $m$ & & $M$ & HC & im \\
\hline 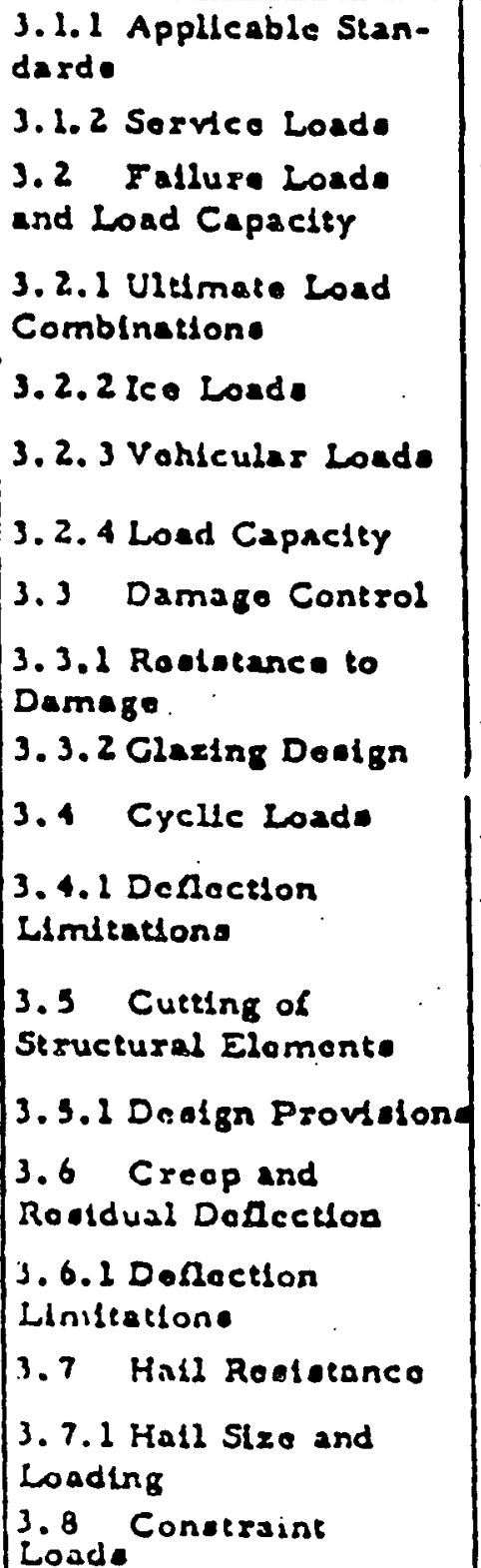 & & $\begin{array}{c}\mathbf{A} \\
\mathbf{N A} \\
\mathbf{A} \\
\mathbf{A} \\
\mathbf{A} \\
\mathbf{A} \\
\mathbf{A} \\
\mathbf{A}\end{array}$ & & 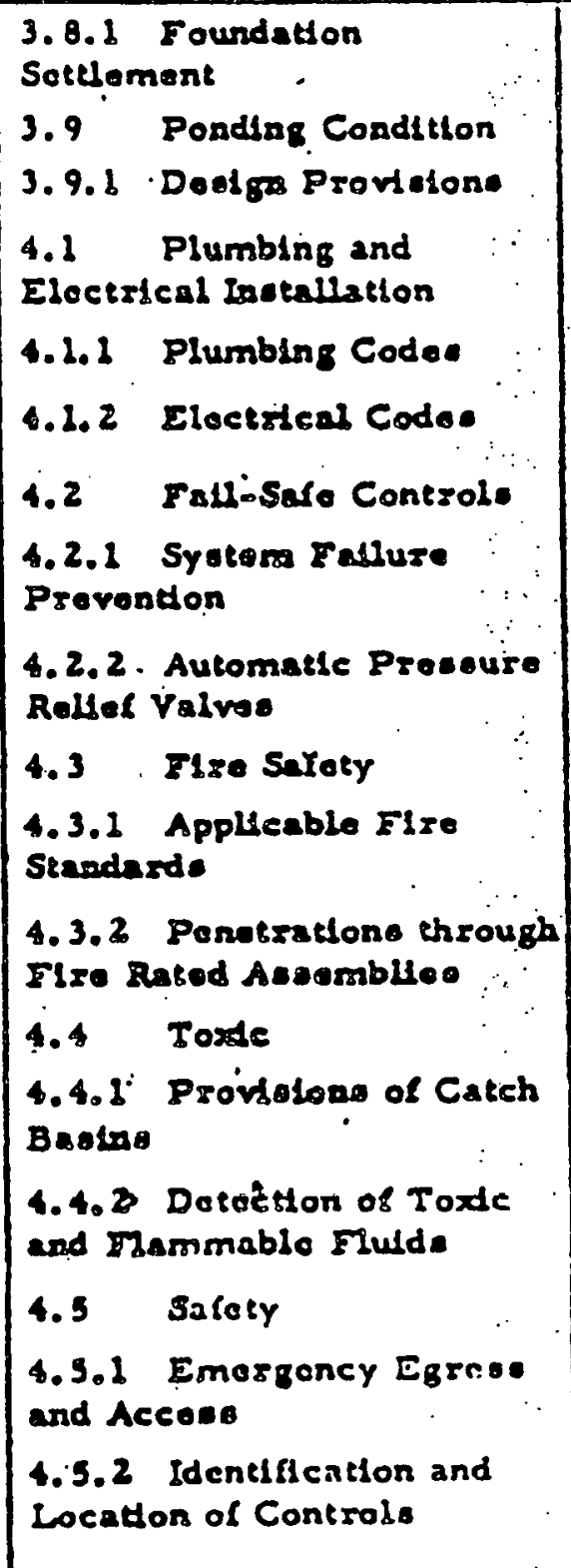 & . & $\begin{array}{c}A \\
A \\
N A \\
\text { NA } \\
\text { NA } \\
\text { NA } \\
\text { A } \\
\text { NA }\end{array}$ & 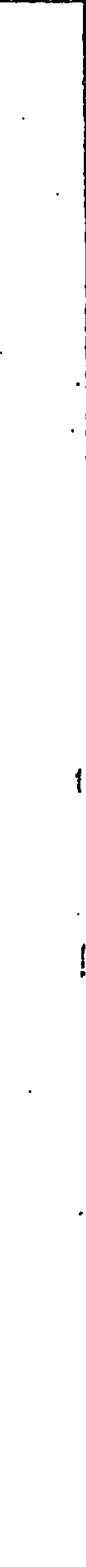 \\
\hline
\end{tabular}




\section{TAFLE 1}

sperisicaiono mon

SHC -3048

TAste. I

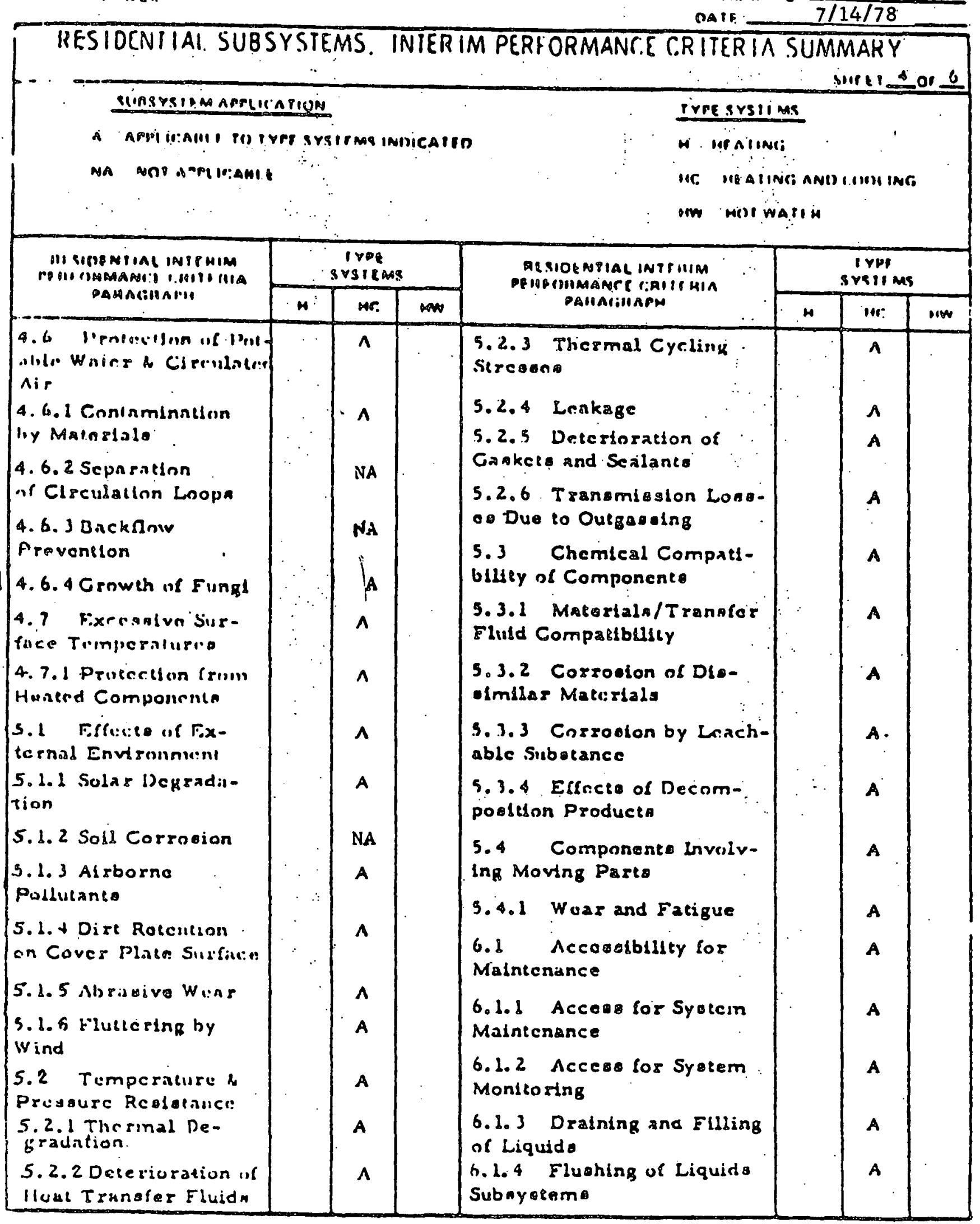


TABLP

sereriention mp. $\frac{\text { SHC }-3048}{2}$

nRVision

BAIt.

$7 / 14 / 78$

RLSIOENTIAL SIJBSYYSTEMS. INTERIM PERFORMANC.E CRITERRIA SUMMARY

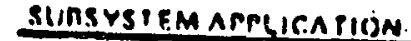

A - applicanie to irpe sysicas innicateo

MA - NOT AaPUr,anLE

$\operatorname{sincet} 5$ oi 6

IVUisYsiLMS

n meating

He - mEATING ANO CIIOLING

WW -. MOT WATEA

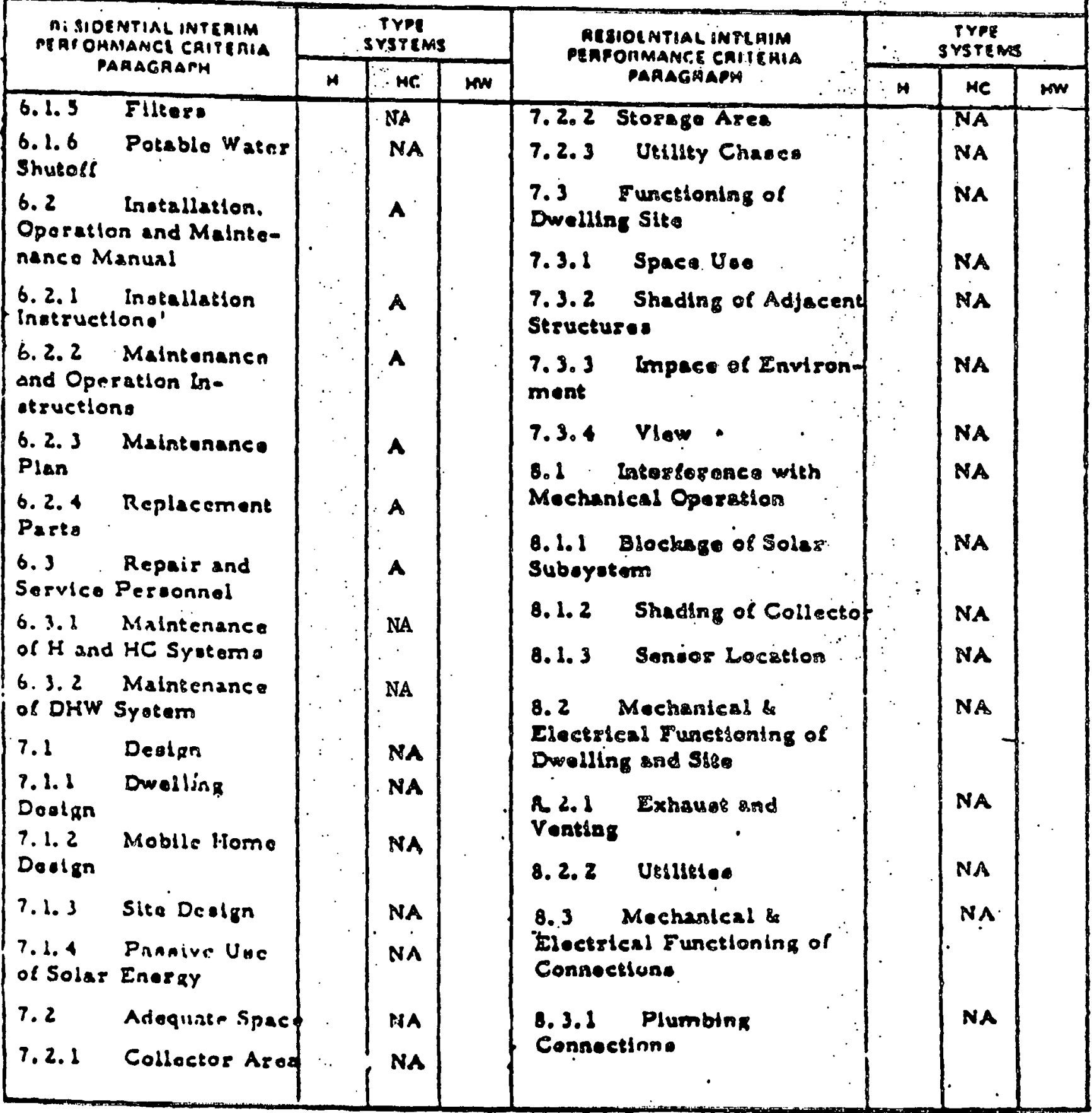


nait $\overline{10-1-76}$

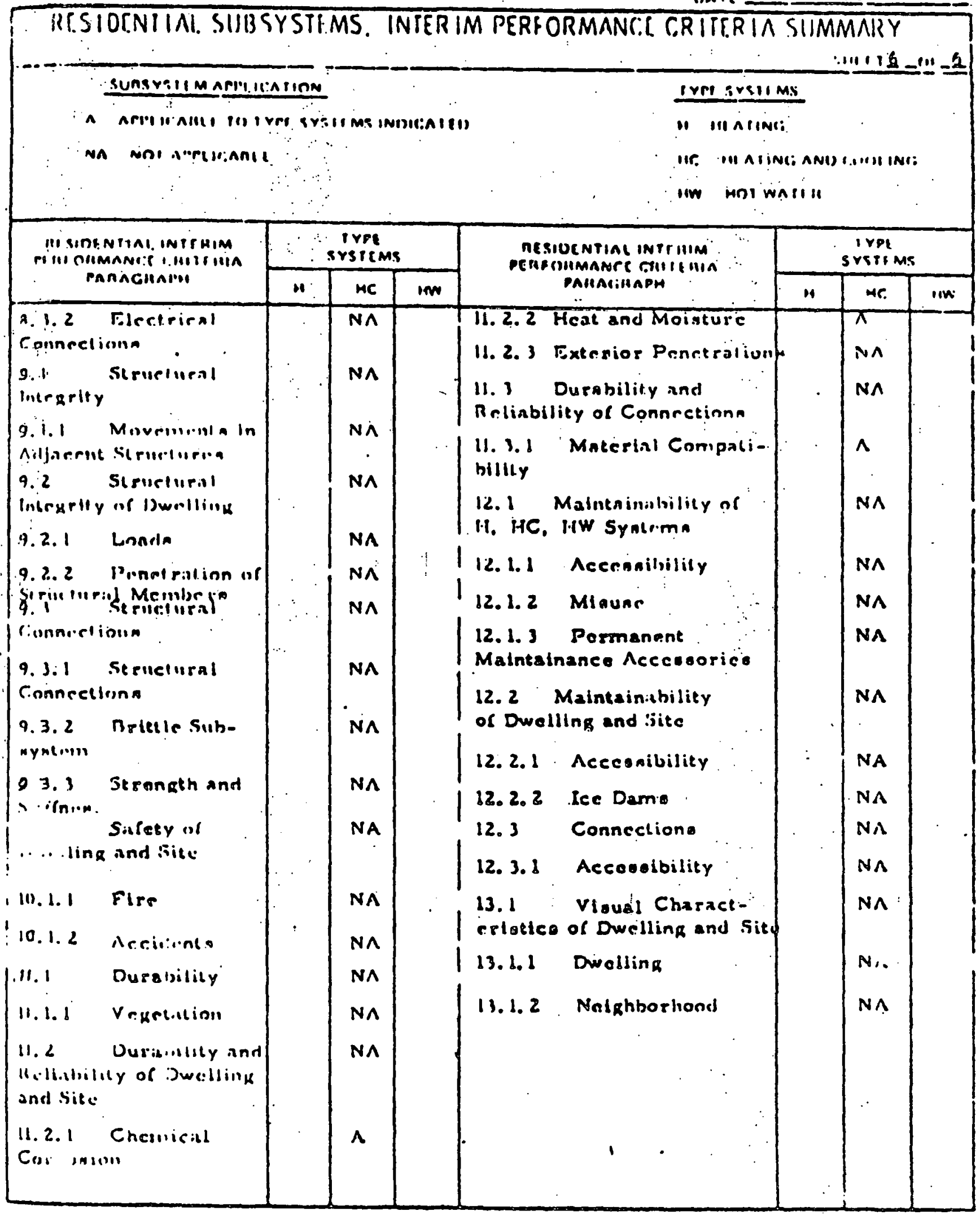


TAOLE "I

SPEeIrICA PION NO.

$54 c-3048$

AEvisinN

Dare $10-1076$

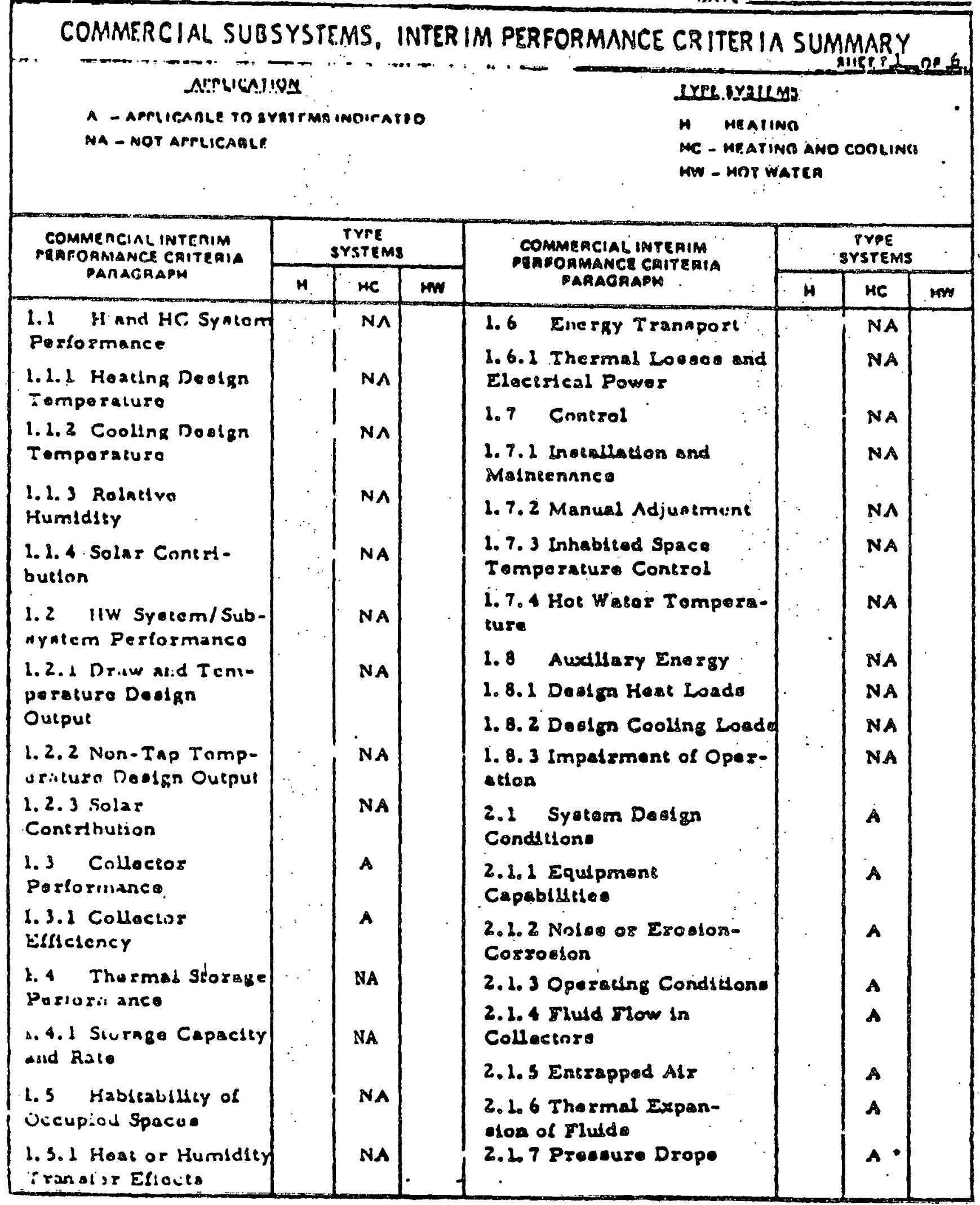




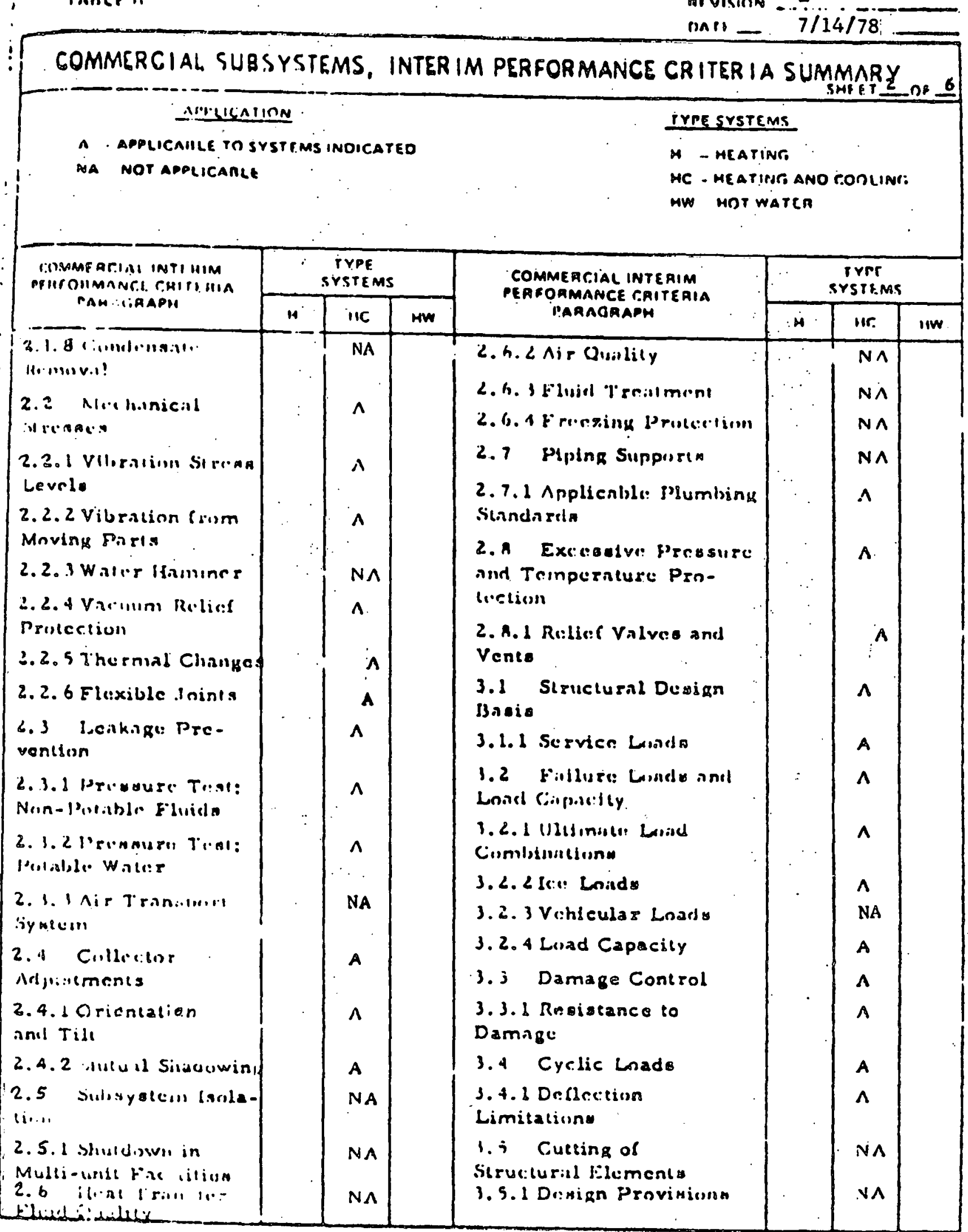


'AOLE II

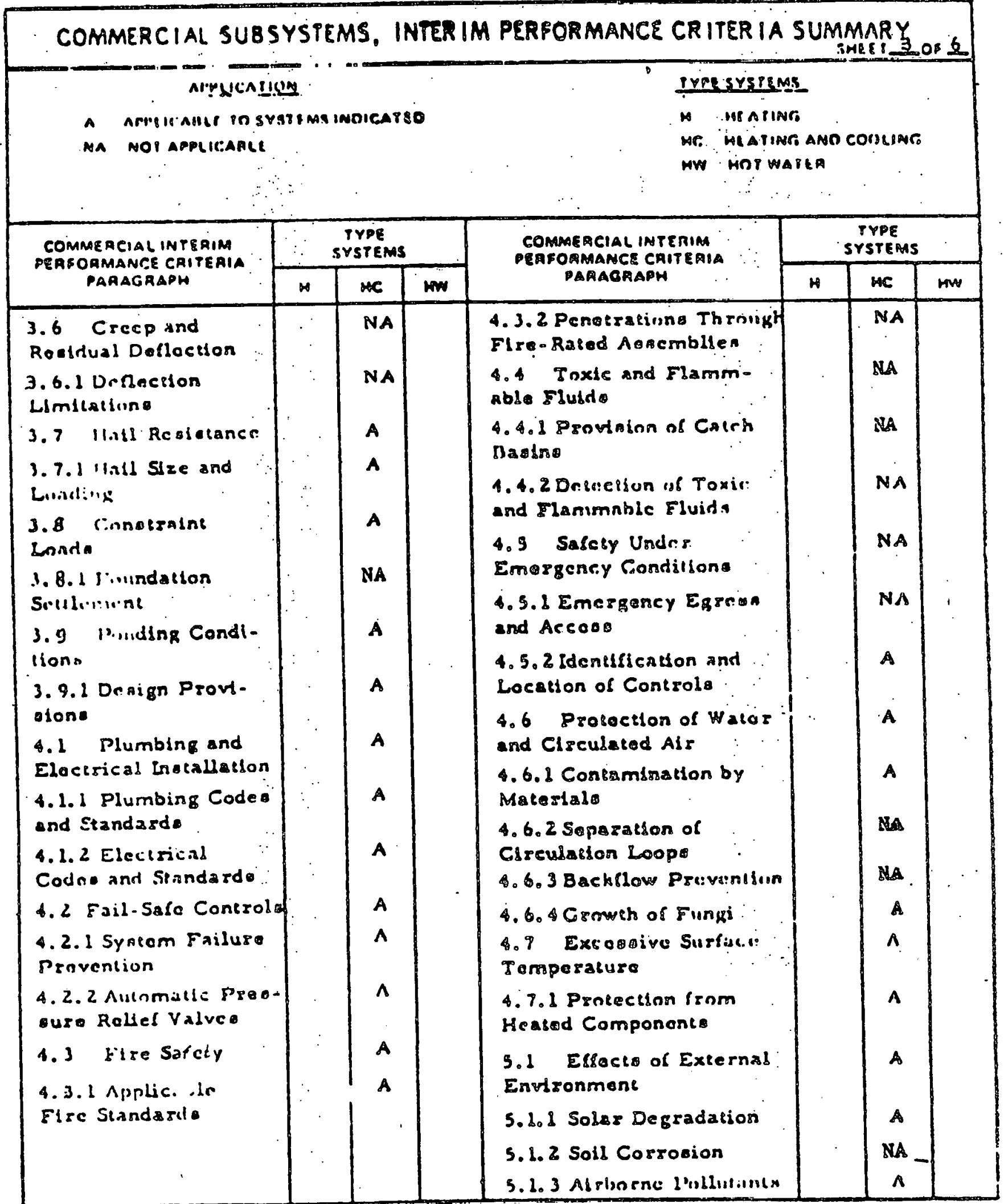



$7 / 14 / 78$

COMMERCIAL SUBSYS IEMS, INTCR IM PERFORMANCE CR ITERIA SUMMARYY

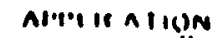

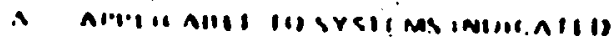

nA NII APIIIIAIII
IYet:5::!!M::

h $116 \wedge$ rim.tr.

ir. in a tionitianis robiline.

IIw IIII watsil

\begin{tabular}{|c|c|c|c|c|c|c|c|}
\hline \multirow{2}{*}{ 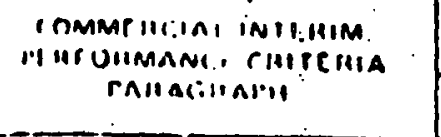 } & \multicolumn{3}{|c|}{$\begin{array}{l}\text { IYrpr } \\
\text { sYsti as }\end{array}$} & \multirow{2}{*}{ 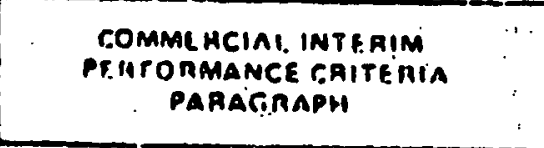 } & \multicolumn{3}{|c|}{$\begin{array}{l}\text { Tripf } \\
\text { EYstf.M: }\end{array}$} \\
\hline & $M$ & HC & $M W$ & & $H$ & HC. & $\mathrm{u}^{1} \mathrm{v}^{-}$ \\
\hline 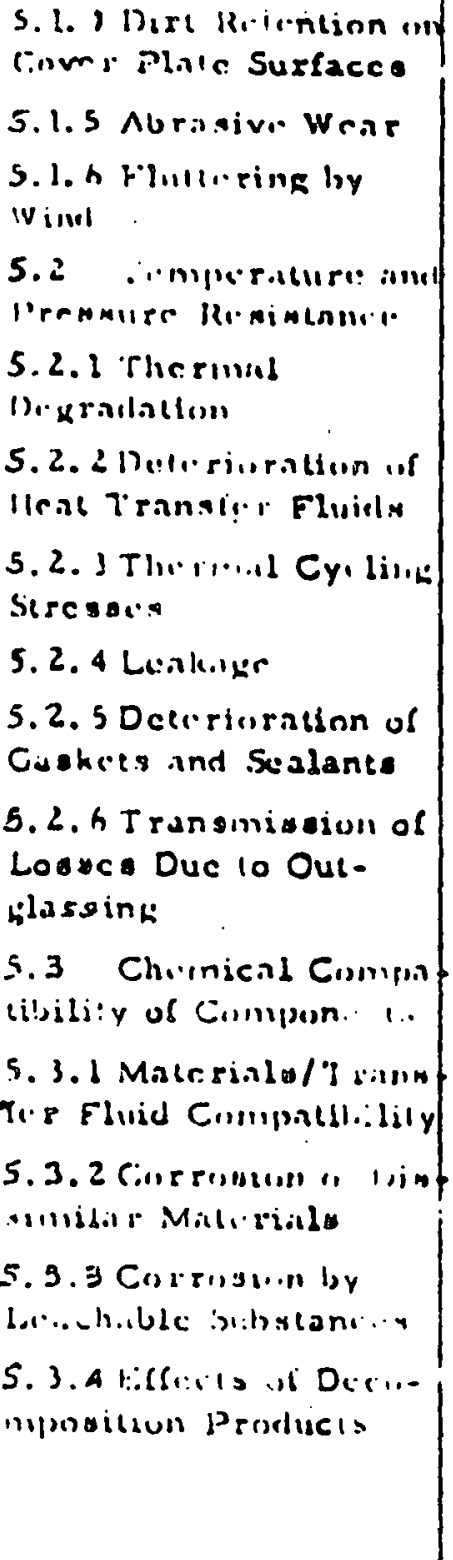 & 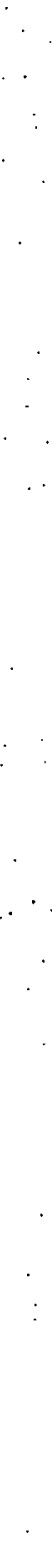 & $\Lambda$ & & 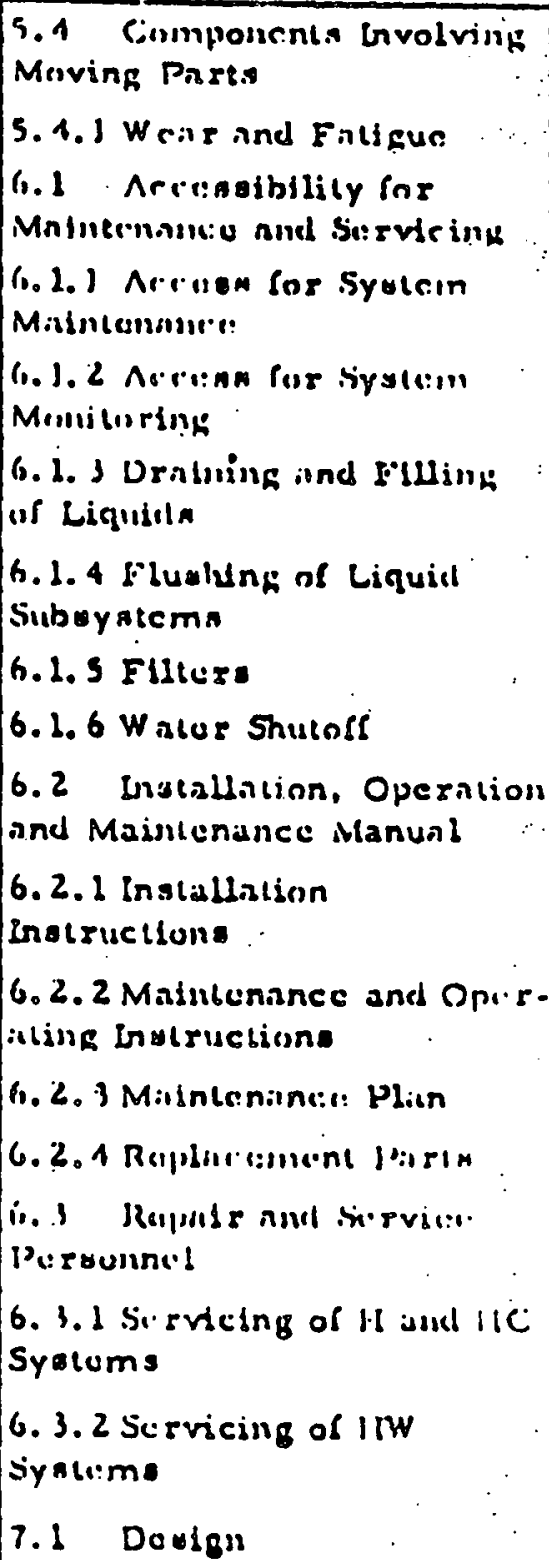 & : & $\begin{array}{l}\text { NA } \\
\wedge A \\
\Lambda \\
\Lambda \\
\Lambda\end{array}$ & v \\
\hline
\end{tabular}


COMMERCIAI SURSYSTEMS, INTERIM PERFORMANCE CRITERIA SUMMARY

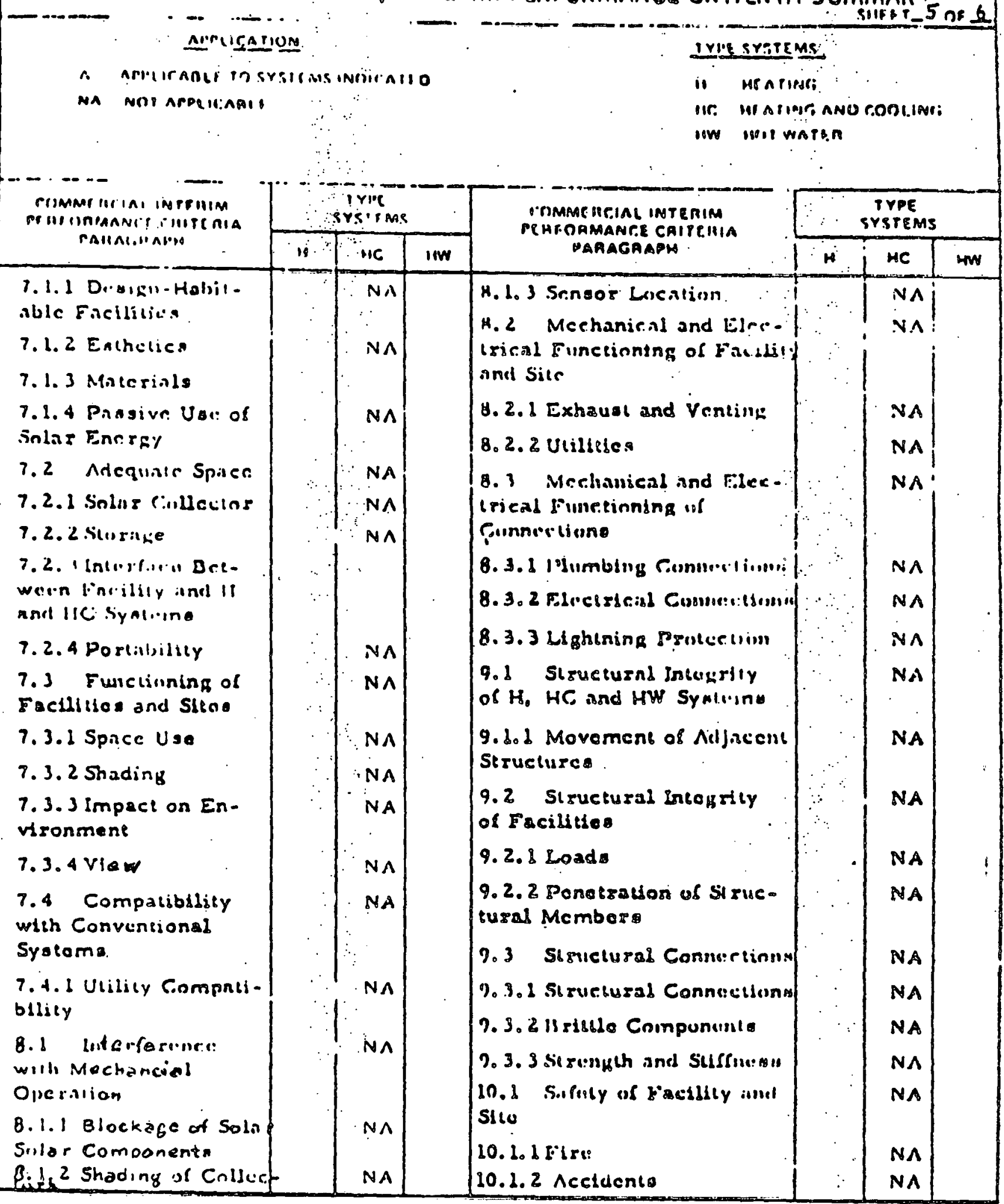


1. COMMERCIAL SUBSYSTEMS, INTER IM PERFORMANCE CRIIERIA SUMMARY Alpiscaninn

1 a armicanir instrsicms indicated

IYUE SYSIEA::

NA NOT APPLIT,AMII.

a miting.

ier. me atini: ant rogilini:

iv WIT matill

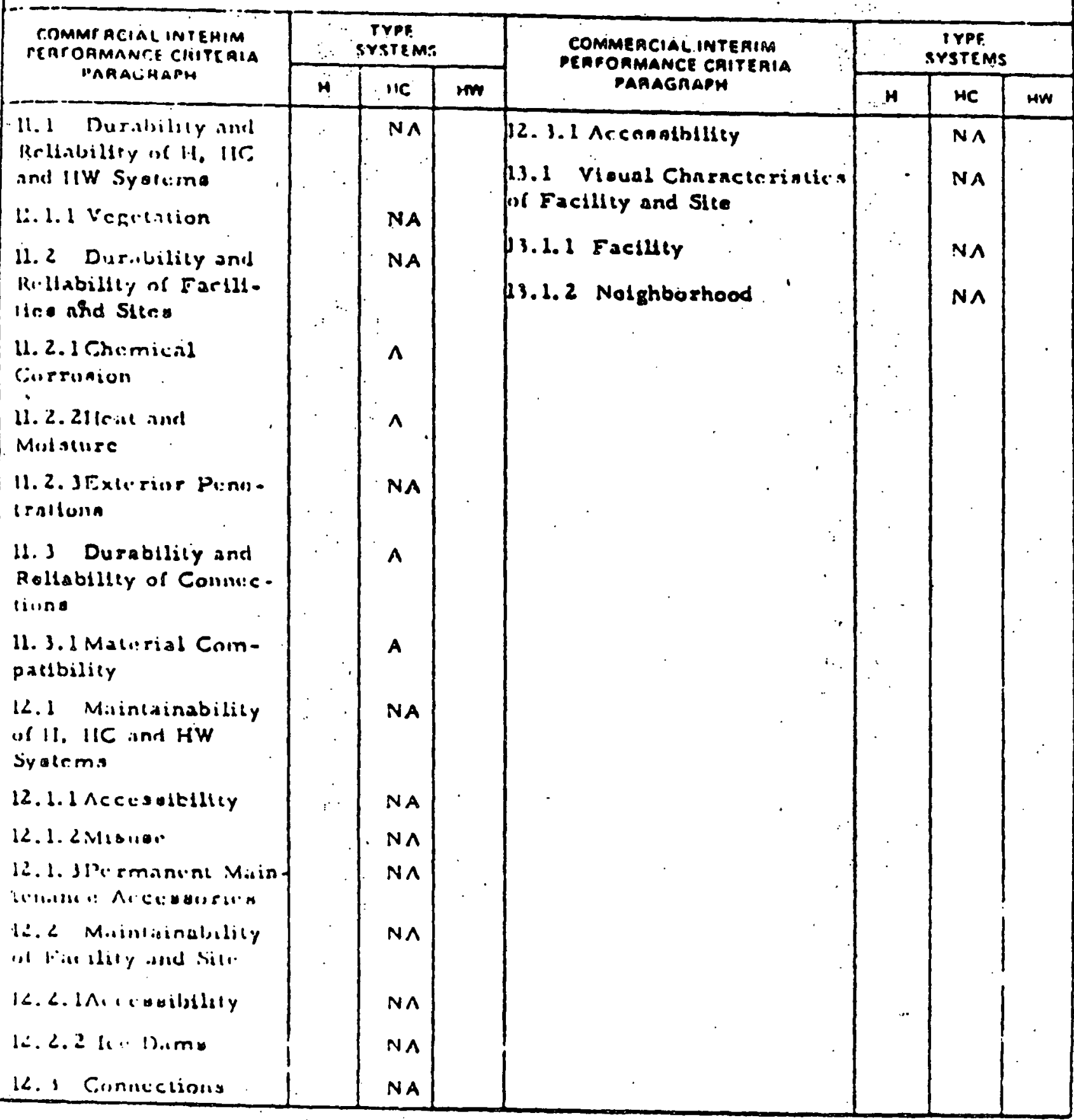




\section{APPENDIX A}

This Appendix defined the thermal performance for collector, Northrup, Inc. Subsystem Model NSC-01-0732, MF-NSC-P "MF" Series.

\section{A-1 SOLAR COLLECTOR}

For Dallas, Texas, the single solar collector will collect a minimum of $1144 \mathrm{BTU} / \mathrm{Ft}^{2} / \mathrm{Day}$ of energy at an inlet fluid temperature of $190^{\circ} \mathrm{F}$ (water). The tilt angle at this performance basis will be equal to the latitude angle, azimuth of 0 degrees, average ambient dry bulb $100^{\circ} \mathrm{F}$, wind velocity zero, August 21 date, direct normal noon solar flux of $283 \mathrm{BTU} / \mathrm{Hr} \mathrm{Ft}$, Iongitude of $97^{\circ}$ and $32^{\circ}$ latitude.

When used primarily for heating, a single collector will provide $1242 \mathrm{BTU} / \mathrm{Ft}^{2}$ Day at an inlet fluid temperature of $150^{\circ} \mathrm{F}$ (water). The tilt angle at this performance bassis is equal to the latitude angle, azimuth of 0 degrees, average ambient $50^{\circ} \mathrm{F}$, wind velocity zero, February 21 date, direct normal noon solar flux of $316 \mathrm{BTU} / \mathrm{Hr} \mathrm{Ft}^{2}$, longitude of $97^{\circ}$ and $32^{\circ}$ latitude.

*ASHRAE Handbook of Fundamentals, American Society of Heating, Refrigerating and Air Conditioning Engineers, Inc., 345 East 47th Street, New York, NY, 1972. 


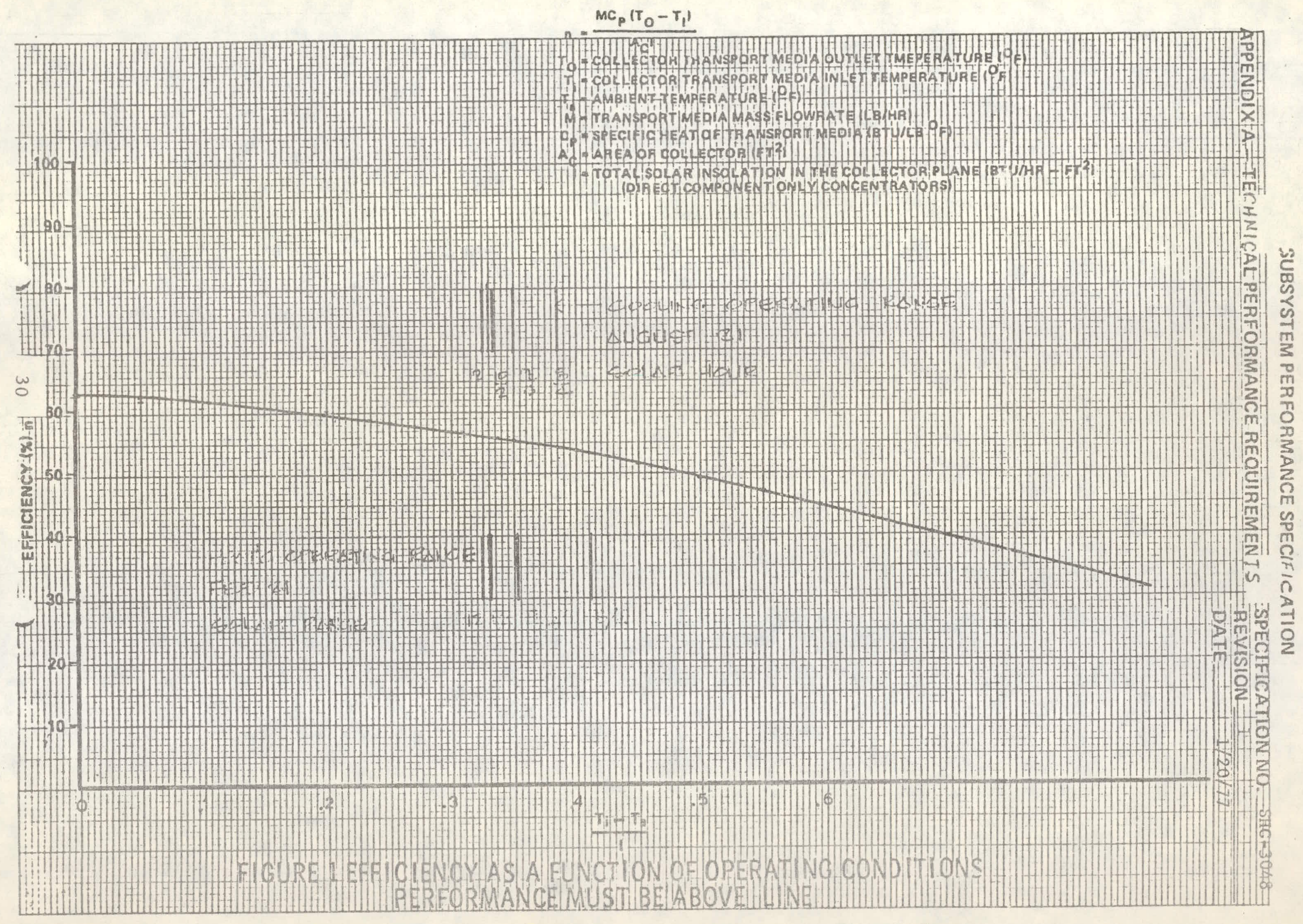




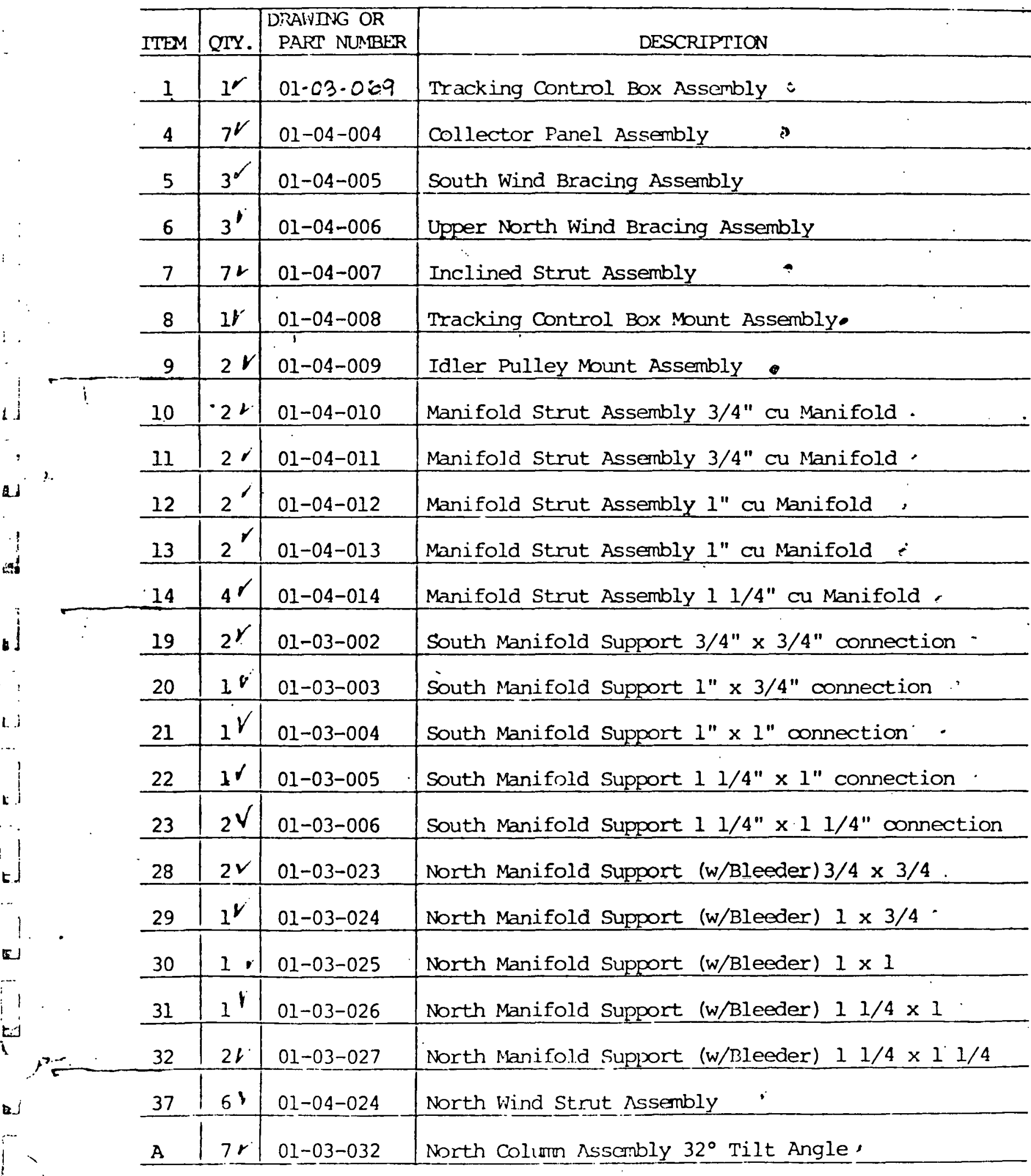


WORMIRUP CCNNENTRATING SOI.AR

DOLIIECTOR PANFIS, MF-NSC-P "ML" SERIES

REV: $B$ 1-.26-78

MODEL NSC-01-0732

NO. 01-05-076

PAGE 2 CF 2

\begin{tabular}{|c|c|c|c|}
\hline ITEM & QTY. & $\begin{array}{l}\text { DRAWING OR } \\
\text { PART NUMBER }\end{array}$ & DESCRIPTION \\
\hline B & $3^{*}$ & $01-04-035$ & Lower North Wind Bracing Assmb. $32^{\circ} \mathrm{Tilt}$ Angle \\
\hline C & 72 & $01-04-044$ & South Brace Assembly $32^{\circ}$ Tilt fingle . \\
\hline $\mathrm{D}$ & $14 r$ & $01-07-003$ & South Column Base Is \\
\hline $\mathbf{E}$ & 1 & $01-04-057$ & Fluid Inlet Assembly $11 / 4$ "Manifold \\
\hline $\mathbf{F}$ & 1 & $01-04-062$ & Fluid Outlet Assembly 1 1/4" Manifold \\
\hline G & 2 & $01-04-065$ & Manifold Fnd Closure 3/4" \\
\hline $\mathrm{H}$ & 1 & $01-04-072$ & Cable Drive Assemble 7 Collector Panels \\
\hline I & $5 \mathrm{ft}$ & $01-13-003$ & Ioose Fill Insulation (1 bag $-7.5 \mathrm{ft} .^{3}$ \\
\hline J' & 1 & $01-04-077$ & Sensor Attachment Assembly \\
\hline
\end{tabular}


INDEX

Products 01 = Solar Collector Panels "ML" Series.

PART NJMBER

01-01-000

01-02-000

01-03-000

01-04-000

01-05-000

00-06-000

00-07-000

00-08-000

00-09-000

$00-10-000$

00-11-000

$00-12-000$

$00-13-000$

$00-16-000$

00-17-000

$00-18-000$

00-19-000

00-20-000

00-21-000

$00-24-000$
DESCRIPTION

Commodities (Steel, Copper Tubing, etc. in SWM (Gen Specs)

Shop Sub-Assemblies

Shop Final Assemblies.

Field Sub-Assemblies

Field Final-Assemblies

Fabricated Sheet Metal Parts

Fabricated Heavy Metal (Struck, etc.)Parts, Extrusicns

Fabricated Tubing and Pipe

Pipe Fittings, Valves, Exp. Comp., Alr Vents, Flow Control Check Valves, Stops, Plugs, Pumps

Electrical Parts, Components, Solenolds, Fabricated Wire, Relays, P/C Boards, Contactors, Motors, Terminals, Wire

Heat Exchangers -- Coils, Etc.

Tanks and Vessels, Accumulators, Expansion Tanks

Insulation

Paints, Coatings, Sealants, Adhesives, Etc.

Hardware - Bolts, Nuts, Screws, Hinges, Bearings, NonElectrical Cable, Clamps, Turn-Buckles, Rivets, Mails, Pins Machine Parts (Spec1al)

Packaging/Shippling

Glass Glazing Coverings

Weather Stripping, Non-Metal1c Gromets, Sponge Rubber, Etc. Tags, Stickers, Labels, Serlal Numbers

*Indicates that no other drawings or specifications other than those contained herein are required.

**Indicates all fasteners, nuts, bolts, and washers are to conform to AlsS (American National Standards Institute). 
01-000-(099) Stee?

$-001$

$-002$

$-004$

$-005$

$-007$

$-010$

$-011$

$-012$

01-100-(199) Copver

$-121$

$-122$

$-123$

$-124$

$01-128$

OH 35-0

01-300-(399) Aluminum

$-301$

$-307$

$-310$

$-311$

$-312$
Steel-Galv.-Dess. G90 (1.25oz/S.F.Commercial)

Fer ASTY A 525; 1526, and A527, subject to ASTY $d-90$

standard method of test. 16 guage, .0636" (.0672 -

.0606 range) $2.650251 \mathrm{bs} / \mathrm{S} . \mathrm{F}$.

Stcel-Galv. Same as 01-01-001, except 20 Guage .0396

(.0425 - .0382 range) 1.65625 lbs/S.F.

Steal-Structural-ASTM A36:

Stecl-Tubing-Cold Drawn-Seamless-dSTM A517, Yechanical Grade

Steel-lialv. - Same as 01-01-001, except 28 Guage .0187 (.0195 - .0.280 range) 0.79125 lbsis.F.

Steel-Channel-3/4" $\times 3 / 8^{\prime \prime} \times 1 / 8 " .54$ 1bs/Ft., Structural A-36

Pipe, Steel, 1!" Nomanal, Sched 40, 2.72 1bs/L.F.

Steel, Rod 3/8", A-36 Struct. Steel.

Tube, Type "M" Hard $5 / 8$

Tube, Type "M" Hard $7 / 8$

Tube, Type "M" Hard $1-1 / 8$

Tube, Type "M" Hard 1-3/8

Refrig Tube ${ }^{1} \mathrm{OOD}$

Refrig Tube $3 / 16$ OD

Aluminum-Bar 5/16" $\times 16^{\prime \prime}-6061-T 6, .551$ 1bs/L.F.

Aluminum, Tubing, 5/8" O.D., .527"I.D., 6061-T6.

Aluminum, Angle, 2" $\times 1$ l. $^{\prime \prime}$ 1/8", 6063-T5, $0.431 \mathrm{lbs} / \mathrm{L} . \mathrm{F}$.

Aluminum, Tubing, 3/8" O.D., .277"1.D., 6061-T6.

Aluminum, Angle, $3 \frac{1}{2} \times 2^{21 "} \times 24 ", 6061-96$

Stand Amer. Shapes, $1.631 \mathrm{lss} / \mathrm{L} . \mathrm{F}$.

$01-500-(599)$ Welding a brazlng Yo[crides

01-500 . Brazine Rou

01-501 ividdin: klectrode 
$\underline{01-01-000}$ COMPODITIES (Continued)

01-600-(699) Lumber, Timber, Plywood \& Other hood Products

$0 i-601$

No. 3 Southern Pine, Dimensioned, 54S, Amercian Std., Spa.

$01-601$

Plywood, Sheath. Grade, DFPA 


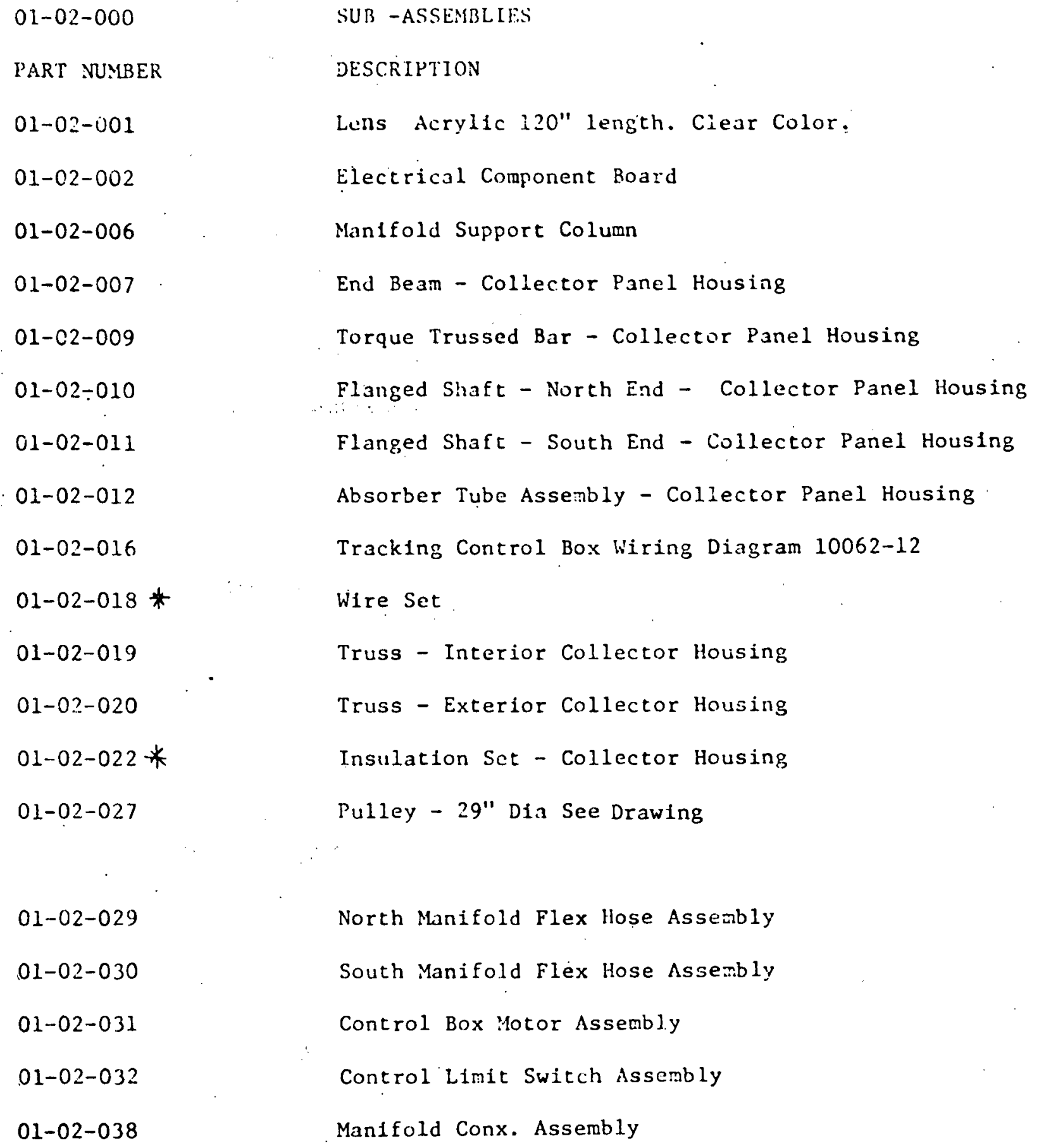


01-03-000

PART NUMBER

01-03-002

01-03-003

01-03-004

01-03-005

01-03-006

01-03-012

01-03-023

01-03-024

01-03-025

01-03-026

01-03-027

01-03-032

01-03-043

$01-03-044$

01-03-045

01-03-046

i:

01-03-047

01-03-048

01-03-049

01-03-050

01-03-051

01-03-053

01-03-055

01-03-060

01-03-063
SHOP FINAL ASSEMBLIES

DESCRIPTIONS

South Manifold Support- $3 / 4 \times 3 / 4$

South Manifold Support - $1 \times 3 / 4$

South Manifold Support - $1 \times 1$

South Manifold Support - $1 \times 1 \frac{1}{6}$

South Manifold Support - $1 \frac{1}{4} \times 1 \frac{1}{4}$

South Brace $\left(32^{\circ}\right)$

North Manifold Support W/Bleeder - 3/4 x 3/4

North Manifold Support - $1 \times 3 / 4$

North Manifold Support - $1 \times 1$

North Manifold Support - $1 \times 1 \frac{1}{4}$

North Manifold Support W/Bleeder - $1 \frac{1}{4} \times 1 \frac{1}{6}$

North Column $\left(32^{\circ}\right)$

Inclined Strut

Manifold Strut - 3/4"

Manifold Strut - 3/4"

Manifold Strut - 1"

Manifold Strut - $1 "$

Manifold Strut i $1^{1}{ }^{\prime \prime}$

Collector Panel Housing

North Wind Strut

End Enclosure - Lens

Solar Sensor Assembly

Absorber Tube

Bleeder Valve Assembly

Fluld Outlet $1 \frac{1}{4} "$ 
01-03-000(Cont 1 nued)

PART NIMBER

01-03-066

01-03-067

01-03-068

01-03-069

01-03-073
DESCRIPTION

Control Box Mount (East aud West)

Idler Pulley Mount Assembly

Inlet \& Outlet Closure Plate $1 \frac{1}{6}$

Tracking Control Box ( 1 " Power Screw)

Closure Plate 


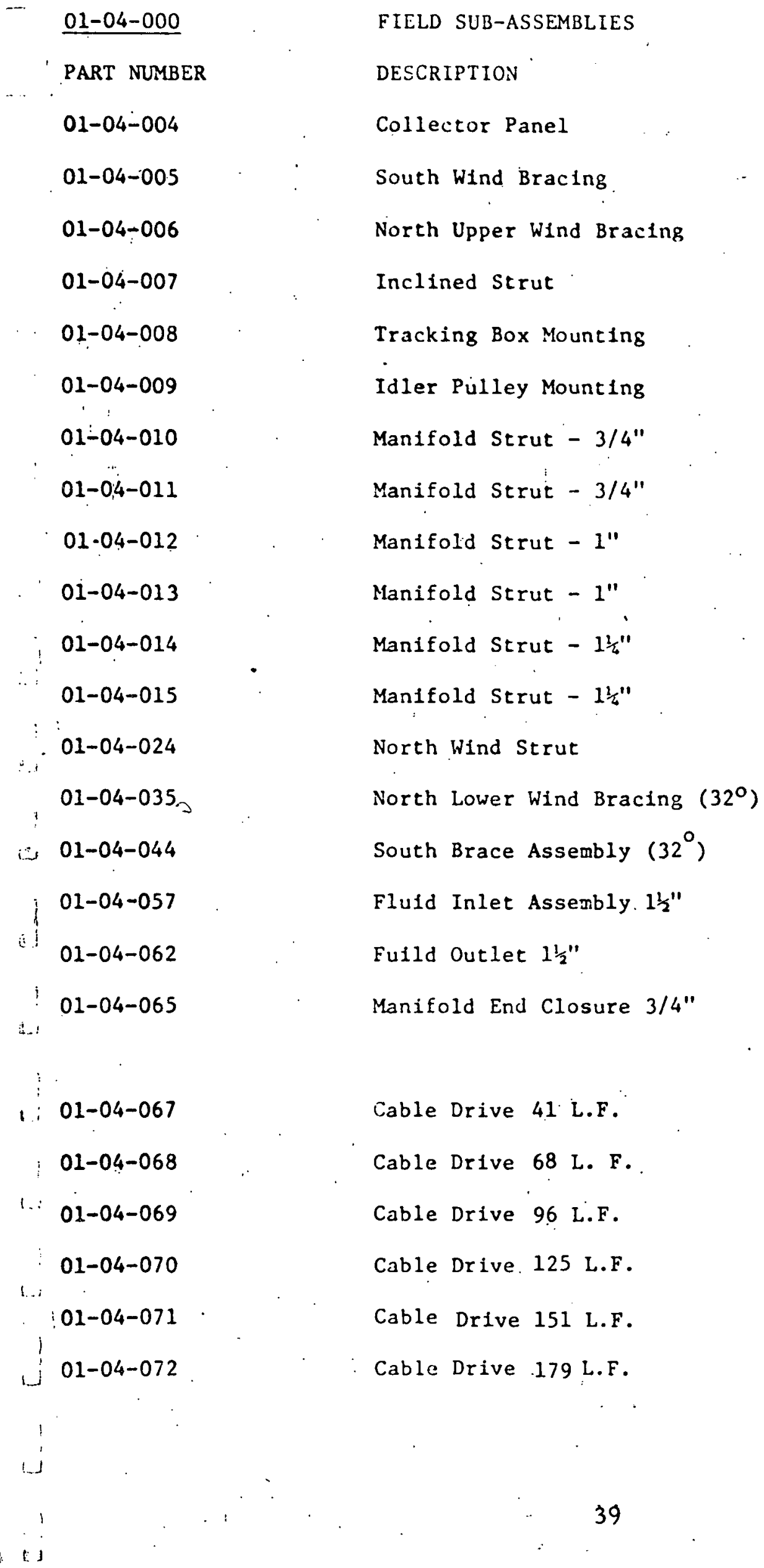




\section{1-04-000 (Cone inued)}
PART NUMBLR
DESCRIPTIUN
$01-04-073$
Cable Drive 206 L.F.
$01-04-074$
Cable Drive 234 L.F.
$01-04-075$
Cable Drive 262 L.F.
01-04-076
Cable Drive 289 L.F.
$01-04-077$
Sensor Attachment TRD-3
01-04-083
Manifold Conx. Assembly $3 / 4 \times 3 / 4$
01-04-084
Manifold Conx. Assembly $1 \times 3 / 4$
01-04-085
Manifold Conx. Assembly $1 \times 1$
01-04-086
Nanifold Conx. Assembly $1 \frac{1}{6} \times 1$
01-04-087
Manifold Conx. Assembly $1 \frac{1}{4} \times 1 \frac{1}{4}$
01-04-088
North \& Scuth Manifold Support Assembly 
01-05-000

PART NUMBER

01-05-007

01-05-076

$01-05-122$

$01-05-123$

01-05-124

01-05-129
FIELD FINAL ASSEMBLIES

DESCRIPTION

Field Assembly - NSC-01-07 (Seven Panel \& Frame)

List of Materials NSC $-01-07$ ( $\left.32^{\circ}\right)$

Field Assembly Section CC

Bolt Settling Plan NSC-01-0732

Spare Parts List

Illustrated Assembly - TYP Array Bay 
$\underline{01-06-000}$

PARTS NUNBER

01-06-002

01-06-003

$01-06-007$

01-06-009

01-06-010

01-06-011

$01-06-022$

01-06-023

$01-06-0.24$

01-06-025

01-06-026

$01-06-027$

$01-06-028$

01-06-029

$01-06-030$

01-06-032

01-06-033

01-06-034
FABTCATED SHET WHAL RARTS

\section{DESCRIPTION}

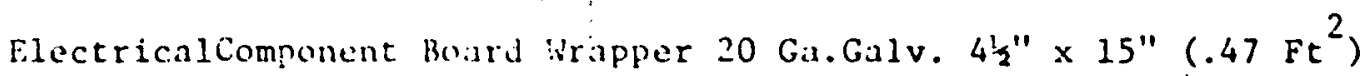
Electrical Component Board Lid 20 Ga. Galv. $4 " \times 3 "\left(.22 \mathrm{Ft}^{2}\right)$ Manifold Strut, End Clip Angle $16 \mathrm{Ga}$. Galv. 3" $\times 4^{\prime \prime}\left(.09 \mathrm{Ft}^{2}\right)$ Capacitor Mounting Strap $20 \mathrm{Gi} .6 \mathrm{alv} .3 / 4^{\prime \prime} \times 47 / 8^{\prime \prime}\left(.03 \mathrm{Ft}^{2}\right)$

Flange stiffener 5", $51 / 8^{\prime \prime}$, s $57^{\prime \prime}$ Wide Struts $16 \mathrm{Ga}$. Galv. $6^{\prime \prime} \times 3^{\prime \prime}\left(.125 \mathrm{Ft}^{2}\right)$

Flange Stiffener with base Angle Connection 16 Ga. Calv. 5" $x$ 6" $\left(.21 \mathrm{Ft} .{ }^{2}\right)$

Fiange Stiffener for 5" lide Struts, See 01-06-010 16 Ga. Galv. $6^{\prime \prime} \times 3^{\prime \prime}\left(.125 \mathrm{Ft}^{2}\right)$

Flange Stiffener for 5" Wide Struts, See 01-06-010:16 Ga. Galv. $3^{\prime \prime} \times 6^{\prime \prime}\left(.125 \mathrm{Ft}^{2}\right)$ lieb Member (Wice) Collectur Truss $20 \mathrm{Ga}$. Galv. $37 / 16^{\prime \prime} \times 137 / 16^{\prime \prime}$
$\left(.32 \mathrm{Ft}{ }^{2}\right)$

Lieb Member (Narro'N) Collector Truss 20 Ga. Galv. 3 3/8" x 13 9/16" (.32 Ft. ${ }^{2}$ )

Cord Top and Botton Collector Truss $20 \mathrm{Ga}$. Galv. 3 3/.4" x 128 3/4" $\left(3.353 \mathrm{Ft}^{2}\right)$

End Strut Collector Truss 20 Ga.Galv. $41 / 16^{\prime \prime} \times 1 l^{\prime \prime}\left(.3 \mathrm{Ft}{ }^{2}\right)$

End Beam, Collector Panel $16 \mathrm{Ga}$. Galv. 16!" $\times 585 / 16^{\prime \prime}\left(6.58 \mathrm{Ft}^{2}\right.$ )

Lens (ivide 20 Ga. Galv. 3 5/8" x 66 13/16" (1.682 Ft. ${ }^{2}$ )

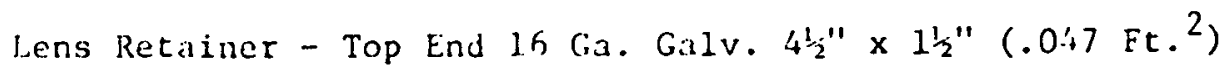

North Manifold/Collector Connect llousing Wrapper 16 Ga.Galv. $111 / 8^{\prime \prime} \times 32^{\prime \prime} \xi^{\prime \prime}\left(1.8 \mathrm{Ft} .{ }^{2}\right)$

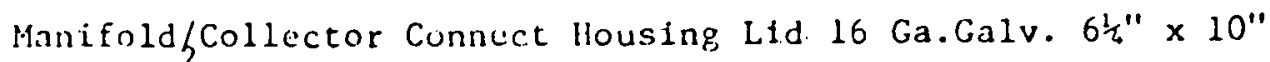
$\left(.43 \mathrm{Ft} .{ }^{2}\right)$

Manifold/Collector Connect Housing Bottom 16 GaGalv. 6" $\times 10 "$ $\left(.42 \mathrm{Ft} .{ }^{2}\right)$ 
PART NUNBER

$01-06-036$

01-06-037

01-06-038

01-06-039

01-06-040

01-06-041

01-06-042

01-06-043

01-06-044

01-06-045

01-06-046

01-06-047

01-06-048

01-06-049

01-06-050

01-06-051

01-06-052

01-06-053

01-06-056

01-06-059

01-06-061

01-06-062

01-06-063

01-06-064
DESCRIPTION

Manifold Support Columin 16 Ga. Galv. 37x" x $15^{\prime \prime}\left(3.9\right.$ Ft. ${ }^{2}$ )

Manifold Strut 16 Ga.Galv. 114! " " $\times 15^{\prime \prime}$ (11.93 Ft. ${ }^{2}$ )

Manifold Strut Cover 16 Ga.Galv. 114 $\gamma^{\prime \prime} \times 6 \frac{2}{4}$ (4.97 Ft. ${ }^{2}$ )

Inclined Strut 16 Ga.Gaiv. 12"' $\times 144^{\prime \prime}\left(12 \mathrm{Ft} .^{2}\right.$ )

Bottom and Side Covering - Collector Panel Housing

26 G. Galv. 42" X 133 5/8" (39.97 Ft. ${ }^{2}$ )

Manffold Positioning Plate 3/4",16 Ga.Galv. 3 7/8" $\times$ 7/8" (.19 Ft. ${ }^{2}$ )

Manifold Positioning Plate 1", 16 Ga.Galv. $37 / 8^{\prime \prime} \times 7 / 8 "$ (.19 Ft. ${ }^{2}$ )

Manifold Positioning Plate $1 \frac{1}{2} ", 16 \mathrm{Ga} . \mathrm{Galv}$.

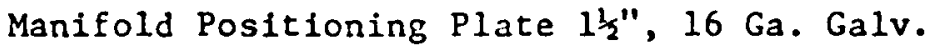

Manifold Posftioning Plate 2", $16 \mathrm{Ga}$. Galv.

Torque Bar-Top Right Hand 16 Ga.Galv. 6" $\times 13315 / 16 "\left(5.581 \mathrm{Ft}^{2}\right.$ )

Torque Bar-Top Left hand 16 Ga.Galv. 6" $\times 13315 / 16^{\prime \prime}\left(5.581\right.$ Ft. ${ }^{2}$ )

Torque Bar-Bottorn 16 Ga.Galv. 6" x 122 15/16" (5.122 Ft. 2)

Truss Connector-Torque Bar-Top 16 Ga.Galv. $13 / 4^{\prime \prime} \times 37 / 16^{\prime \prime}\left(.042 \mathrm{Ft}^{2}\right)$ Torque

Truss Connector-Bar-Bottom 16 Ga.Galv. $13 / 4 " \times 59 / 16^{\prime \prime}\left(.068\right.$ Ft. ${ }^{2}$ )

End Connector-Trussed Torque Bar 20 Ga.Galv. $41 / 16^{\prime \prime} \times I^{\prime \prime}\left(.028 \mathrm{Fi}^{\circ}{ }^{2}\right)$

Flange Stiffener-Wind Bracing Connector 16 Ga.Galv. $3 \times 6$ (.125 Ft. ${ }^{2}$ )

Outlet Closure Plate - Blank 16 Ga.Galv. 4" $\times 7^{\prime \prime}\left(.2 \mathrm{Ft} .^{2}\right)$

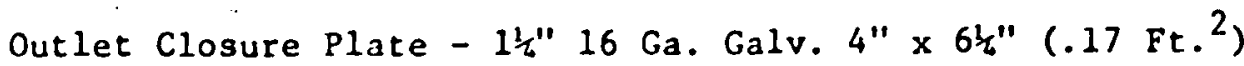

Control Box Mount $16 \mathrm{Ga}$. Galv. 12" $\times 26^{\prime \prime}\left(2.17 \mathrm{Ft} .^{2}\right.$ )

Br1dg1ng-Cross-Collector Panel 16 Ga.Galv. 9/16" $\times 15 \frac{1}{2} "\left(.061 \mathrm{Ft}^{2}\right.$ )

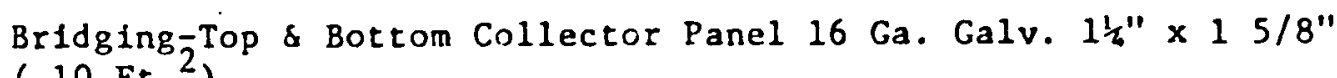
$\left(.10 \mathrm{Ft}{ }^{2}\right)$

Bridging-Vertical Collector Panel 16 Ga.Galv. 1 5/8" $\times 11^{\prime \prime}\left(.12 \mathrm{Ft} .^{2}\right)$

Bridging-Connector-Torque Bar, Right Hand, Collector Panel

$16 \mathrm{Ga}$. Galv. $15 / 8^{\prime \prime} \times 1 "\left(.011 \mathrm{Ft} .{ }^{2}\right)$ 
01-06-000 (Cont inued)

PARTS NUMBER

$.01-06-069$

$01-06-075$

01-06-079

$01-06-080$

01-05-081

01-06-082

01-06-083

01-06-104

01-06-107

01-06-109

01-06-110

01-06-111

$01-06-112$

$01--6-122$

01-06-123

$0.1-06-124$

01-06-125

01-06-126

01-06-127

01-06-132

$01-06-133$
DESCRIPTION

South Brace $\left(32^{\circ}\right) 16$ Ga.Galv. $123 / 8^{\prime \prime} \times 263 / 16^{\prime \prime}\left(2.25 \mathrm{ft} .^{2}\right)$

South Manifold Connect Housing hrapper. 16 Ga. Galv. 11 1/8" $\times 23 \frac{1}{\prime \prime}$ (1.8 $\mathrm{Ft} .{ }^{2}$ )

Bridging Connector-Torque Bar-Le?t Hand, Collector Panel

16 Ga.Galv. $15 / 8^{\prime \prime} \times I^{\prime \prime}\left(.011 \mathrm{Fr}^{2}\right)$

South Brace (320) See 01-03-012. 16 Ca. Galv. $123 / 8^{\prime \prime} \times 263 / 16^{\prime \prime}$ $\left(2.25 \mathrm{Ft}^{2}\right)$

Idler Pulley hrapper 16 Ga.Galv. 5' $\times 1611 / 16^{\prime \prime}\left(.58 \mathrm{Ft}^{2}\right)$

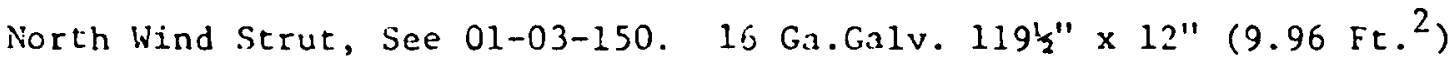

North Coluinn $\left(32^{\circ}\right)$. See 01-03-032. 16 Ga. Galv. 3" $\times 6^{\prime \prime}\left(.125\right.$ Ft. $\left.{ }^{2}\right)$

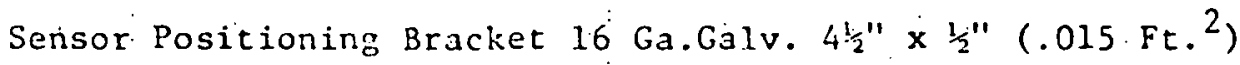

Cap-Idler Pulley Mount $16 \mathrm{Ga}$. Galv. $51 / 16^{\prime \prime} \times 57 / 8^{\prime \prime}\left(.21 \mathrm{Ft}{ }^{2}\right)$

lligh Limit Switch U-Fastener 16 Ca.Galv. $23 / 8^{\prime \prime} \times 2 \frac{1}{2} "\left(.041 \mathrm{Ft} .{ }^{2}\right)$

Solar Sensor Mount $16 \mathrm{Ga}$. Galv. 5" $\times 7^{\prime \prime}\left(.252 \mathrm{Ft}{ }^{2}\right)$

Absorber Tube Retalner $16 \mathrm{Ga}$. Galv. $23 / 16^{\prime \prime} \times 33 / 4^{\prime \prime}\left(.06 \mathrm{Ft}{ }^{2}\right)$

End Plate. See 01-03-051. $16 \mathrm{Ga}$. Galv. $51 / 8^{\prime \prime} \times 12^{\prime \prime}\left(.427 \mathrm{Ft} .^{2}\right)$

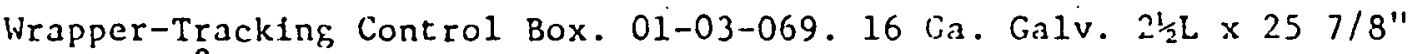
(3.86 Ft. ${ }^{2}$ )

End Panel-Tracking Control Box. 01-03-069. South End. 16 Ga.Galv. $7 \frac{1}{4} " \times 815 / 16^{\prime \prime}\left(.45 \mathrm{Ft} .^{2}\right)$

End Panel-Tracking Control Eox. 01-03-069. North End. 16 Ga. Galv. $7 !^{\prime \prime} \times 815 / 16^{\prime \prime}\left(.45 \mathrm{Ft}{ }^{2}\right)$

Bracket-Bearing Box. 01-03-069. 16 Ca.Galv. $113 / 4^{\prime \prime} \times 57 / 8^{\prime \prime}$ $\left(.48 \mathrm{Ft} .{ }^{2}\right)$

Cover for Limit Swltch-Tracking Control Box 01-03-069, hest Side. 20 Ga.Galv. 4 1/8" $\times 5$ 13/16". (.17 Ft. $\left.{ }^{2}\right)$

Cover for L1mlt Switch-Tracking Control Box 01-06-069, East Side 20 Ca. Galv. $41 / 8^{\prime \prime} \times 513 / 16^{\prime \prime}\left(.17 \mathrm{Ft} .^{2}\right)$

i'ulley Cable Clamp Plate. 16. Ga.Galv. $\frac{1}{2} " \times 1 \frac{1}{2} "(.005$ FL .2)

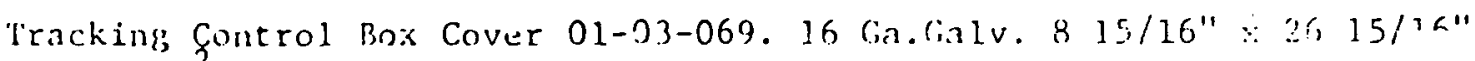
$\left(1.67 \mathrm{Ft}^{2}\right.$ ) 
01-06-000 (Continued)

PARTS NUMBER

$01-06-134$

01-06-135

01-06-136

$01-06-137$

$01-06-138$

01-06-162
DESCRIPTION

SHIM Control Box, Left Side, 16 Ga. Galv. STL. 4 5/8" x 3" (.096 Ft. ${ }^{2}$ ) SHIM Control Box, Right Side, $16 \mathrm{Ga}$. Galv. STL. $45 / 8^{\prime \prime} \times 3^{\prime \prime}\left(.096 \mathrm{Ft}{ }^{2}\right)$ MTG Bracket- Motor (01-03-069 Control Box) 16 Ga.Galv. 3" x 5 1/8"
(.11 Ft. ${ }^{\text {") }}$

Lens End Closure Weather Strip Plate. $20 \mathrm{Ga}$. Galv. $41 / 16^{\prime \prime} \times 12$ " 9.346 Ft.2)

Lens Gulde-Splicer. $20 \mathrm{Ga}$. Galv. STL $37 / 16^{\prime \prime} \times 3^{\prime \prime}\left(.072 \mathrm{Ft} .^{2}\right)$

Flange Stiffener. $16 \mathrm{Ga}$. Galv. 3" $\times 6 \frac{1}{2} "\left(.135 \mathrm{Ft} .{ }^{2}\right.$ ) 
01-07-000

PARTS NUMBER

01-07-003

01-07-017

$01-07-023$

01-07-024

01-07-025

01-07-026

01-07-027

$01-07-028$

01-07-029

$01-07-030$
FARRICAILD IILAYY YFTA. (STRUCTURAL, ETC.) PARTS

DESCRIETION

South Col. Base Angle, 3's" $\times 22^{\prime \prime} \times 4 "$ x. 2'-11" 6061-T6 Alum. 1.691 Lbs/L.F. $(01-312)$

Flange-Shaft Collectur Panel ASIM A-36 Structural STL." $\frac{1}{2}$ thick Plate.

Housing-Power Screw, East Side, Control Box, 01-03-069

Housing-Power Screw, hest Side, Control Box, 01-03-069

Pulley Rim, See Drawing. 3/4" $\times 3 / 8^{\prime \prime} \times 1 / 3^{\prime \prime} \times 104^{\prime \prime}$ AST. A-36 Structural steil Channel .54 1bs/Ft.

Pulley Top Spoke, Sec Drawing. 3/4" $\times 3 / 3^{\prime \prime} \times 1 / 8^{\prime \prime} \times 1317 / 32$ ASTM A-36 Structural Steel.

Pulley Bottom Spoke, See Drawing. 3/4" $\times 3 / 8^{\prime \prime} \times 1 / 8^{\prime \prime} \times 1317 / 32^{\prime \prime}$ ASTM $\Lambda-36$ Structural Stecl

Pulley llusizontal Spoke, See irawing. 3/4" $\times 3 / 8: \times 1 / 8^{\prime \prime} \times 1317 / 32^{\prime \prime}$ ASTM $\Lambda-36$ Structural Steel

Pulley Hui, See Drawing. Schelilis 40 black Steel Pipe x 13/8" Length.

Puli.ey Mount Angle, See Drawing. 2" $\times 1 "$ × $1 / 8^{\prime \prime} \times 0^{\prime}-2 ! "$ Alum. Angle, 6063-T5. 
01-08-000

PARTS NUMBER

01-08-002 *

$01=08-003-k$

01-08-004 *

01-08-005*

01-08-006*

01-08-011*

01-08-012*

01-08-017*

01-08-018*

01-08-019*

01-08-020*.

01-08-021 *

01-08-023*

01-08-024*

a)

01-08-025 *

01-08-026 *

1.!

01-08-027 *
FABRICATED TUBING \& PIPE

DESCRIPTION

Mantfold 3/4" X 119 11/32" Type "M" cu.

Manifold $3 / 4 "$ " $1183 / 4 "$ Type "M" eu:

Mantfold I" X 119 3/32" Type "M" cu.

Manifold I" X $1187 / 16$ " Type "M" cu.

Manifold 1! $\frac{1}{4}$ " $\times 11813 / 16$ Type "M" cu.

Tee, Bleeder Connecting Type "M" cu. 4" $x$ 3

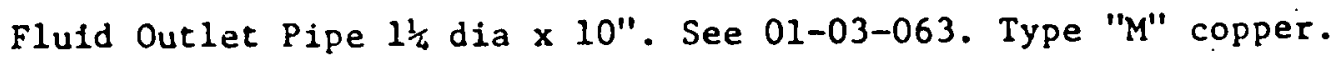

Maid-0-Mist Lead Tube $\frac{1}{2}$ " Type "L" $x$ 5"

Pressure Relief Valve Lead Tube, $3 / 4 "$ x 2 " "M"

Schrader Valve Connect Tube $3 / 16$ O.D. "L" $\times 4 \frac{1}{2}$

Soft Dr. Copper (00-01-135)

Absorber Tube ( $w /$ tubes \& caps) Exterior, Type " $M$ " copper. See 01-02-012.

Absorber Tube (w/tubes \& caps) Interior, Type " $M$ " copper. See 01-02-012.

Absorber Tube Manifold Pipe, Exterior 5/8" $0.0 \frac{2}{1}$ "nom x 6.313" Type "M" Copper

Absorber Tube Manifold Pipe, Intertor 5/8"O.D. $\frac{1}{2} "$ nom $x 6.125$ Type " $M$ " Copper

Absorber Tube Manifold Pipe North 5/8" O.D. 1/2" nom $\times 5.0 "$ Type " $M$ " Copper.

Absorber Tube Manifold Pipe, South 5/8" 0.D. $\frac{1}{2 "}$ nom x 3.625" Type "M" Copper.

Absorber Tube Mantfold Riser 5/8" O.D. $\frac{1}{2}$ " nom x 2 13/32" Type "M" Copper 
01-09-000

PART NUMBER

01-09-001 *

01-09-002

01-09-004 *

01-09-005 *

01-09-006 *

01-09-007*

01-09-008 *

01-09-009 *.

01-09-010 *

01-09-011

01-09-012

01-09-014 *

01-09-016 *

01-09-017 *

01-09-018 ㅊ

01-09-019 *

01-09-020*

01-09-021 *

01-09-022 *

01-09-024 *

01-09-060 나

01-09-002 *
PIPE FITTINGS, VALTES, EXP. COM., AIR VENTS, FLOW CONTROL, CK VALVES, STOPS, PLUCS, PUIPS, $\mathrm{H}_{2} \mathrm{O}$ ACCESSORIES.

DESCRIPTION

Maid-O-Nist ifi. W/S" FET suto-Vent, Air Eliminator

Pressure Relief Valve hitts $3 L, 125$ PSl Release, ASME; $3 / 4 "$ MPT Connector

Swivel Conn. GRA-TEK

Schrader Valve Insert

Adapter Cu. Tube $3 / 3 " 0 . D ., " M P T$, Cast Brass

Cap - Cu. Tuid, Wrought Copper, $13 / 8$ " O.D.

Wrought Tee $1 ; \times 3 / 4 \times 3 / 4$

Wrought Tee $\frac{1}{2} \times 1 \times 3 / 4$

Wrought Tee $\frac{1}{2} \times 1 \times 1$

Wrought Tee $!_{2} \times 1 \times 1 !_{4}$

Wrought Tee $\frac{1}{2} \times 1 \frac{1}{4} \times 1 !$

Wrought Tee $\frac{1}{2} \times 1 ! \frac{1}{2} \times 1 ! 2$

Flex Hose CRA-TEK

Alapter, ivrought cu., 3/8" O.D. Sweat $x$ l/" MPT

Alapter, wrought cu., 7/3"O.D. Sweat $\times 3 / 4 "$ MPT

Wrought TEe $\frac{1}{2} \times \frac{1}{2} \times \frac{1}{2}$.

$I_{5}$ Nom. Shore Rad. (Ells) $90^{\circ}$ Elbow, Wrought

$\frac{1}{2}$ Nom. Wrought Coupling W/Stop Lec

$\frac{1}{2}$ Nom. Male Adapter, Wrought

Schrader Viave $\frac{1}{4} " \phi$ Flare Adapter

Adapter Wrot., 5/8" $\times \frac{1}{2} "$, LEE $\# 15-101$ or eqial (MPT)

Plug. Wrot. Cu. to lit $7 / 8 "$ O.D. Fitting 
01-10-000

PART NUMBER

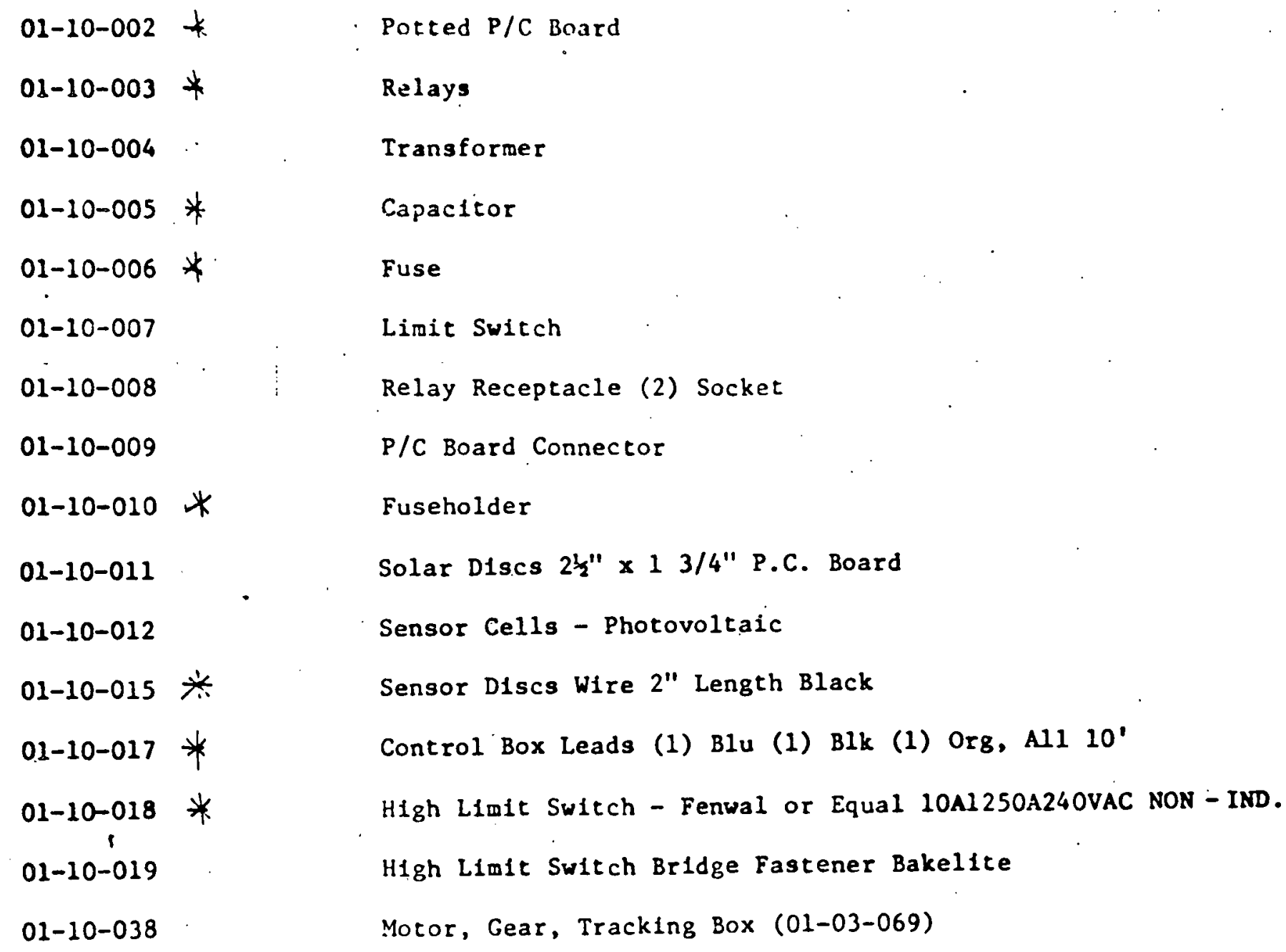

ELECTRICAL PARTS, COMPONENTS,' SOLENOIDS, FABRICATED WIRE, RELAYS, P/C BOARDS, CONTACTORS, MOTORES, TERMINALS, WIRE

DESCRIPTION

or, Gear, Tracking 
00-13-00

PART NUMBER

01-13-002*

01-13-003 $*$

01-13-004 *

01-13-005

01-13-006 苂

01-13-007 ‡

\section{INSULATION}

DESCRIPTION

Owens Corning Flberglass Insulation, l" Thk, RA 22

Fiberglasspouring insulation, Owens Corning $1 \mathrm{Bag}=7.5 \mathrm{Ft} .^{3}$

Batt - $1000^{\circ} \mathrm{F}$ Owens Corning AFU Solar $1000^{\circ}$

$\because \times 2 \frac{1}{2}$ Du Pont Microform Insulation

! $\times 37 / 8$ Du Pont Microform Insulation

1" Thk Smooth Foil Back Duet Board Insulation 
$\underline{00-16-000}$

PART NUMBER

00-16-001

00-16-002

$00-16-003$

00-16-004 *
PAINTS, COATINGS SEALANTS, ADHESIVES, ETC.

DESCRIPTION

Z.R.C. Metal Conditionter

Z.R.C. Zinc Coating

Z.R.C. Zinc Coating. Thinner

Selective Coating, NASA Black Chrome, Olympic

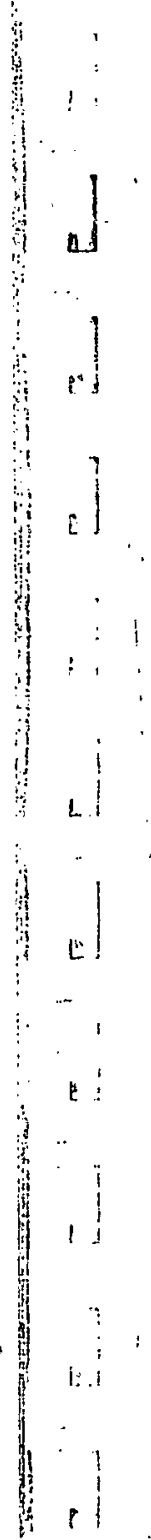


$\underline{01-17-000}$

PARTS NUNRER

01-17-002

$01-17-003$

$01-17-004$

$01-17-008$

01-17-009

01-17-010

01-17-011

$0.1-17-0.12$

01-17-013

$01-17-014$

01-17-015

01-17-016

01-17-019

01-17-021

01-17-022

01-17-027

01-17-028

01-17-039

01-17-030

01-17-035

$01-17-036$

01-17-037

01-17-039

01-17-041

01-17-044

$01-17-045$
ILIRDVARE -- BULTS, NUTS, SCIEWS, IILNGES, EEARINGS, NONELECTRICAL CABLE, CLATPS, TURN-BUCKLES, NAILS, PINS

\section{DESCRIPTION}

Serew \#4 $x$ ! (40 Tl' I ) Zinc

Stand off $\frac{1}{4}$ O.D. $\times \frac{1}{2}$, Threaded (40 TPI) Alum.

$\# 6 \times 11 / 8 "$ (32 TPI), Slotted Head, Zinc Coated

$\# 8 \times \frac{1}{2}$, S.D. \& S.T. (Tek) Screw, 18 TPI

Pulley 12" O.D. $\times 11_{2}^{\prime \prime}$ Bore with Cable Clamp Bale

$3 / 8 \times 3 / 4^{\prime \prime}$ Hex Bolt, Zinc Coated

3/8" Hex Nut, Zinc Coated

$3 / 8^{\prime \prime}$ Lock Washer, Zins Coated

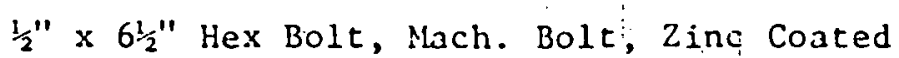

$\frac{1}{2} "$ Hex Nut UNC, Zinc Coated

$\frac{1}{2}$ " Lock Washer, Zinc Conted

Bearing $1 \frac{1}{2} "$ Cast Iron, Set Screw

Cable Clamp $\frac{1}{4} \phi$

SPLIT Lock Washer (\$6) Zinc Coated

Nut, Hex \#6 (32TPI) Zinc Coated

Bolt, Machine Hex Head, $\frac{1}{4} " \times \frac{1}{2} "$, INC. Zinc Coated

Nut, Hex Head ' $\frac{4}{4}$ UNC, Zinc Coated

Lock. Washer " 7", 7inr Conted

Bolt Spacer ! $2 \phi \times 5 / 8$ "

Cable, Aircraft, Calv. $7 \times 7,4 "$ Dia. Preformed

Bolt Spacer $\frac{1}{2} "$ I.D. $x$ 74/8", Alum. Tube (01-307)

Bolt Spacer $\frac{1}{2}=5$, Alum. Tube $(01-307)$

Bolt Spacer !! $\frac{1}{2} 53 / 16$ Alum. Tube (01-307)

$\frac{1}{2}$ Flat Washer $12 \mathrm{Ga}$.

Pop Rivets 1/8" Alum. $x \frac{1}{6} "$ length

Pin, !" $\times 2 !$, Hex H.D. 
PARTS NUYBER

01-17-046

01-17-049

01-17-050

01-17-051

01-17-052

01-17-053

01-17-054

01-17-056

01-17-057

$01-17-058$

01-17-059

01-17-060

01-17-061

01-17-062

01-17-063

$r$

01-17-065

01-17-066

01-17-067

1.: $01-17-072$

01-17-073

$01-17-074$

$21-17-075$

01-17-076

$01-17-077$

$01-17-078$

01-17-079

$01-17-080$
DESCRIPTION

Heymian Mfg. Co. Wire - Ty W/Screw Hole. -3 Screw

Bolt Carrlage $3 / 86 \times 3 / 4 "$ UNC (UNC)

Flange $(1 ! x) \quad 4-25-172$

Nut, Hex 3/8"\$ -16 TPI Zinc Coated

3/8"\& Lock Washer Zinc Coated

Bearing (1 $\left.\frac{1}{i}\right)$ Bearing

Locking Collars ( $1 \frac{1}{2}$ ) Bearing

Fastner-Lock $\frac{1}{2} "$ (T1mmerman) C12007-017, or Equal

Bolt-Mach. $-\frac{1}{6} \times 2 \frac{2}{6}$ Hex H.D.

Spacer Bolt $-\frac{1}{2} \times 111 / 16$, Alum. Tube (00-01-311)

Spacer Bolt - $\frac{1}{2} \times 1$ 13/16, Alun. Tube (00-01-311)

\#6 - $32 \times 3$ 1/8" Screw R.N. HD, Zinc Coated

$\$ 6-32$ Hex Nut

Bolt Hex $\frac{1}{2} \phi \times 1 / 2$ UNC, Zinc Coated

Fastener Lock $\frac{1}{4} \times 4$ (Tfmerman) or Equal

$5 / 8$ Nut Zinc Coated

$5 / 8$ Bolt $\times$ lis 21 nc Coated

Lock Washer (5/8) Z1nc Coated

$3 / 8$ Flat Washer Z1nc Coated

Cable Thimble $\frac{1}{2}: "$ ZInc Coated

Turnbuckle $3 / 8 \times 6 "$, Galv., Equal to Upson Walton H-62GJJ Galv. Whth 2-114 Lock Nuts

$\frac{1}{4} \times 20$, Hex Nut, ZInc Coated

l冖" Star Lock Washer, ZInc Coated

Turnbuckle for Wind Bracing, H31, 3/8\$ $\times$ 6" Upson/WaltonGalv. (No Eye, Hook or Jaws)

1440 TPI $\times \frac{1}{2} "$ Bolt, Z1nc Coated

$\$ 440$ Nut, Zinc Coaced

it 4 lock wasibe, Zine Coated 
PARTS NLMBER

$01-17-081$

$01-17-082$

. 01-17-083

00-17-095

00-17-096

$00-17-098$

00-17-099

$00-17-100$

$00-17-118$

$00-17-119$

1

$00-17-120$

$00-17-121$

$00-17-122$

$00-17-123$

01-17-129

$01-17-130$

\section{DESCRIPTION}

$46 \times \frac{1}{2}, 32$ TPI Slotted Round Head, Z1nc Coated

$\$ 6$ Hex Nut, 32 TPI, Zinc Cuated

46. Star Lock Washer, Zinc Coated

ł" D1a. x ł", Mach. Bolt, Slotted, Rd. H.D., Unc., Zinc Coated

Ł." D1a., Hex Nut, UNC. Zinc Coated

Wind Brace Lower, 3/8"6 x 2'-0" A36 STL Rod W/ 6" Left Hand Thread, 3/8-16, Galv. After Fabrication.

North (Lower) End, Wind Brace, Upper, 3/8 $69^{\prime}-4^{\prime \prime}$ A36 Stl. Rod W/3/8" $\phi-16$ TPI $\times 0-6 "$ R. H. Thread. Galv. After Fabrication

North \& South (Upper) End Wind Brace, Upper, 3/8"d $\times 7^{\prime}-10^{\prime \prime}$ A36 St. Rod W/3/8 - 16TPI x 0'-6" R. H. Thread, Galv. After Fabrication

Bearing Flanged Cast Housing (Two Bolt) NTN Toyo Brg. Co. \#UCFL 209, 1 3/4 I.D.

Sprocket, 3/8" Bore for No.' 35 3/8" Pitch American Std. Roller Chain, Browning No. 359.

Sprocket, $13 / 4$ Bore for No. 35 3/8" P1tch, American Std. Roller Chain, Browning No. 35845

Roller Cha1n, 3/8" P1tch No. 35 Single Strand

PIn St1. 光", Hex $\times 3^{\prime \prime}$

Brazing Rod (01-500)

Steel Retainer Clip for Swivel

Screw, \$8x 5/8, Hex HD, With Meoprene Washer Bonded to Metal Cap, S.D \& S.T: Equal to TEK 
$\Gamma \quad \underline{01-18-000}$

PARTS NUNBERS

$01-18-005$

$01-18-006$

01-18-009

$01-18-010$

01-18-014
MACHINED PARTS (SPECI.IL)

DESCRIPTION

Shaft-North End Collector Panel, Cold Drawn Seamless STL Tubing ASTM $5191 \frac{1}{2}$ " O.D. $x 0.109$ Wall Thk $\times 37 / 8$ " Length

Shaft-South End Collector Panel Cold Drawn Seamless STL Tubing ASTM $5191 \frac{1}{2} "$ O.D. $\times 0.109$ Wall Thk $\times 2 \frac{1}{2} "$ Length

Shaft - $1 \frac{1}{4}$ Dia. $x 0.109$ wall Thk'ness Cold Drawn Seamless Steel Tubing ASTM 519 Mech. Grade $x$ 9! Length

Power Screw and Nut, Tracking Control Box 01-03-069

Cable Guide (01-03-069 Control Box) 
$\underline{00-19-001}$

PARTS NUNBER

01-19-008*
, PACKAGING/SHIPPING

DESCRIPTION

Carton for NASA Prototype 


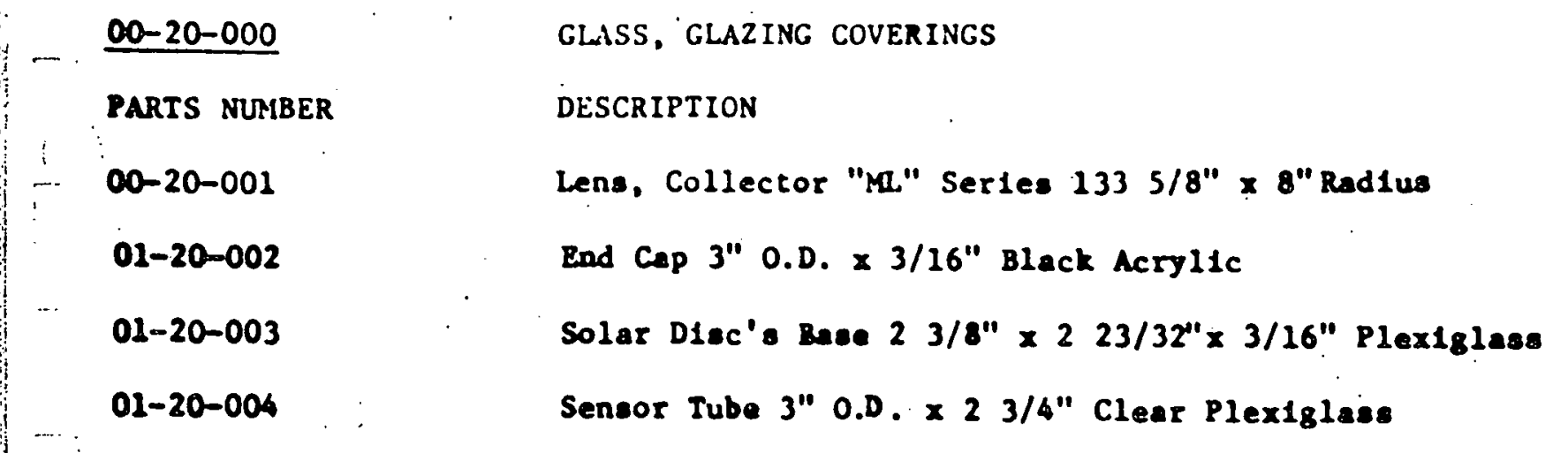


$01-21-000 *$

PARTS NUMBER

$01-21-001$

$01-21-004$

$01-21-005$

01-21-006

$01-21-019$

$01-21-021$
WHTHER STRIPPINC, NON-YEEALIC GROMMETS, SPONGE RUBBER, ETC. DESCRIPTION

Gromets "Rubber" Atlantic India, \#2856 or Equal

Weatherstripping self-adhesive, $3 / 4 \times 3 / 16$ Cleotex

Gromet North Manifold Atlantic India \#2903 or Equal

Silicon Sealant Equal to GE RTV White

Silicon Sealant Equal to GE RTV Black

Viton "O" Rin's 


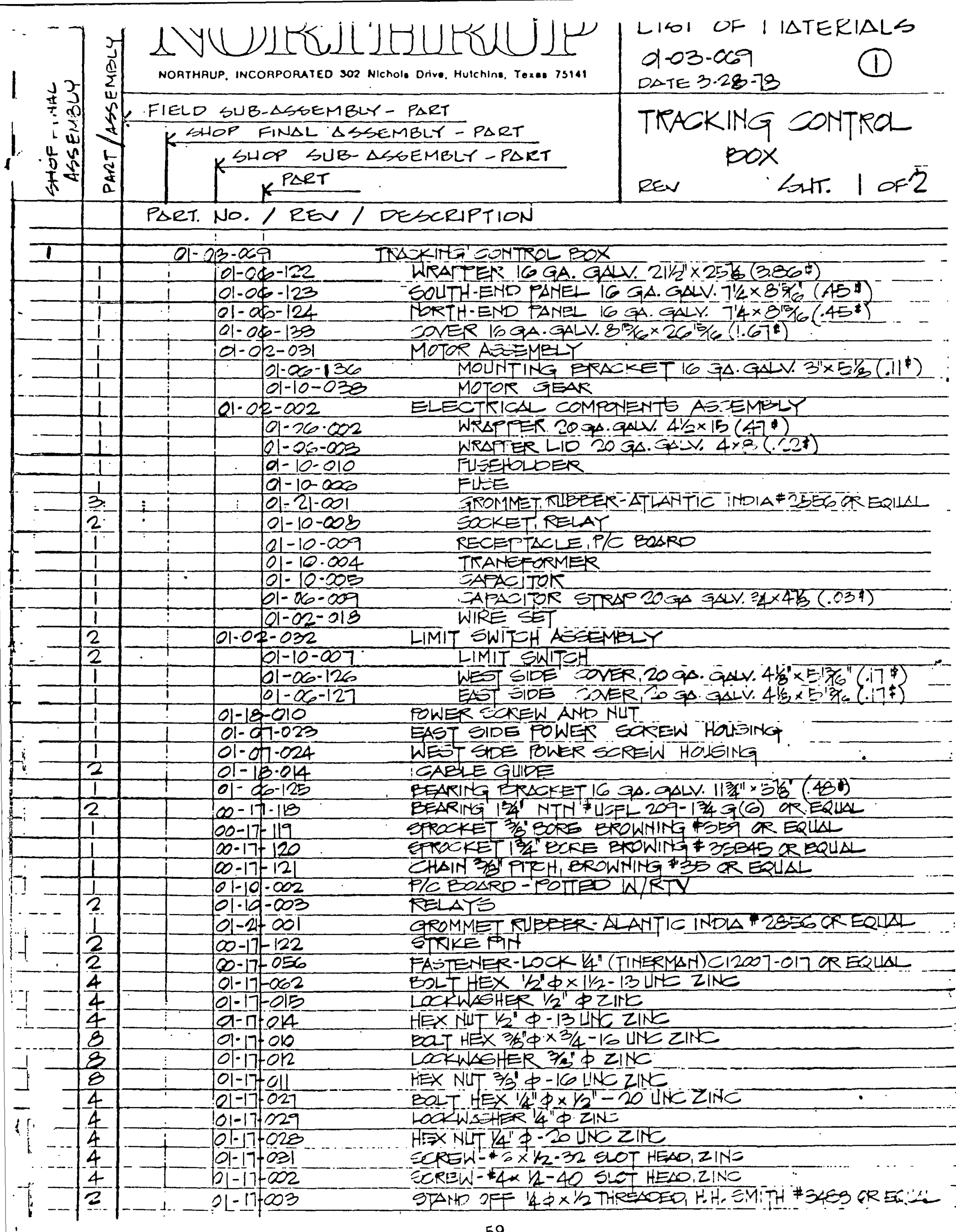




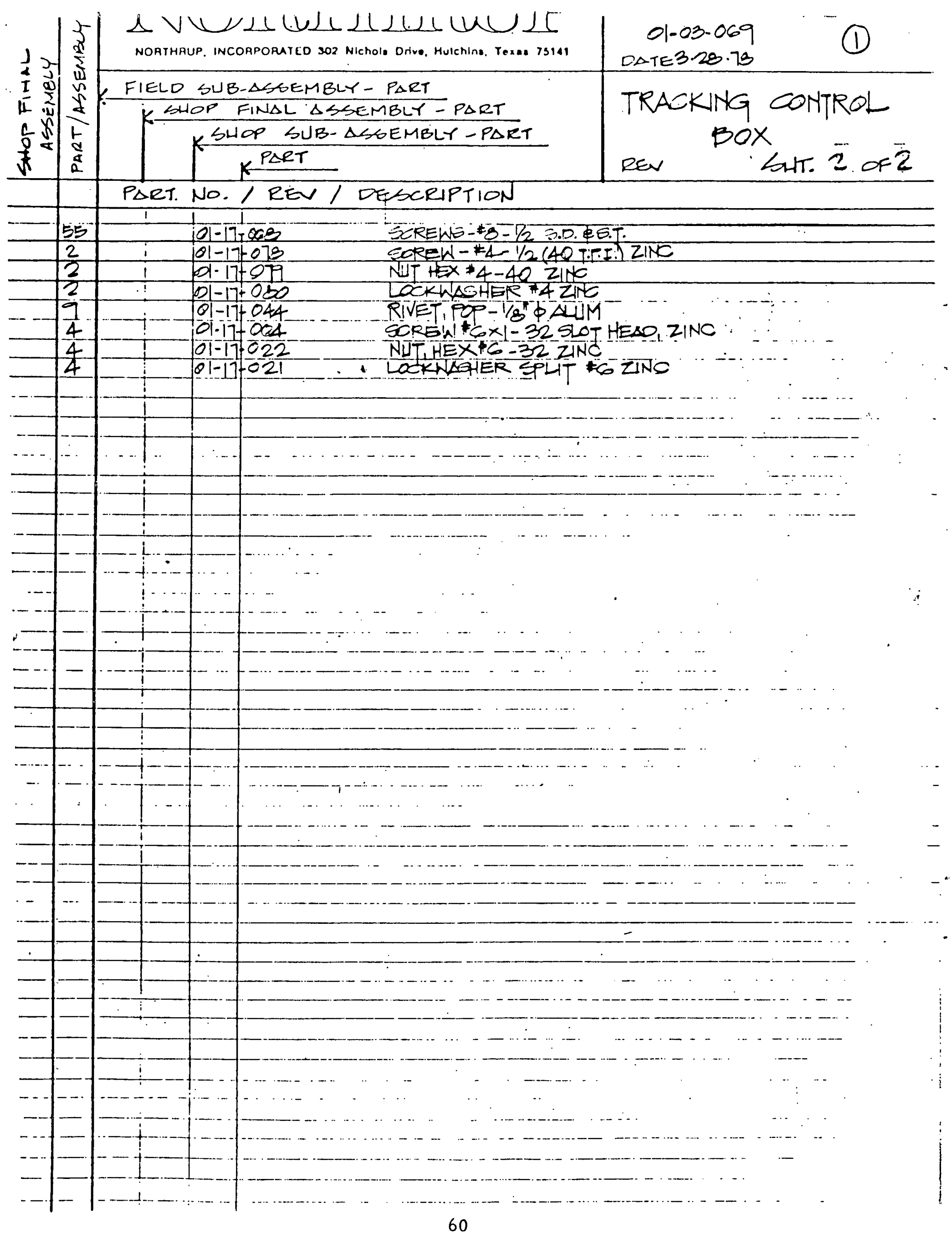




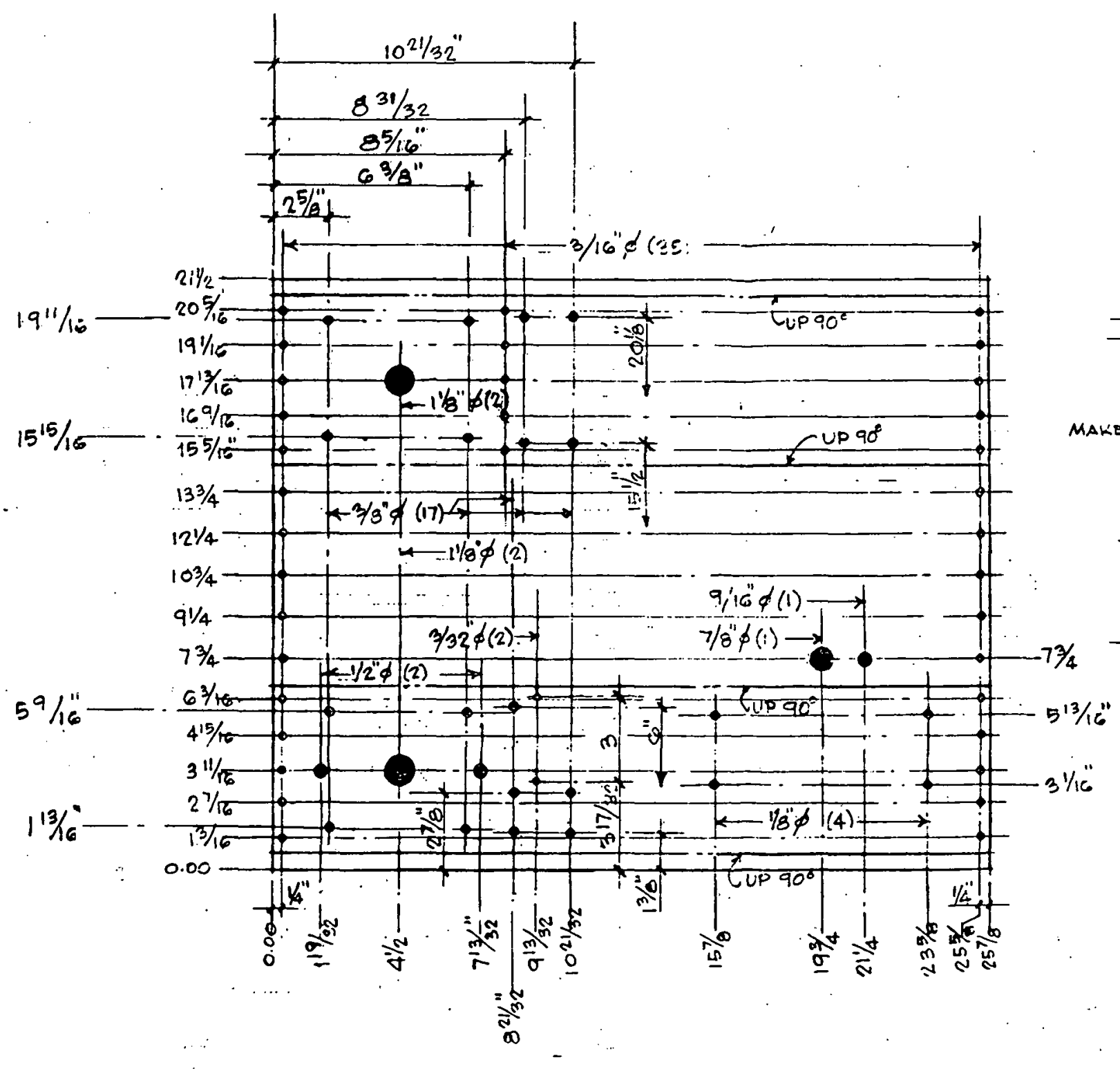

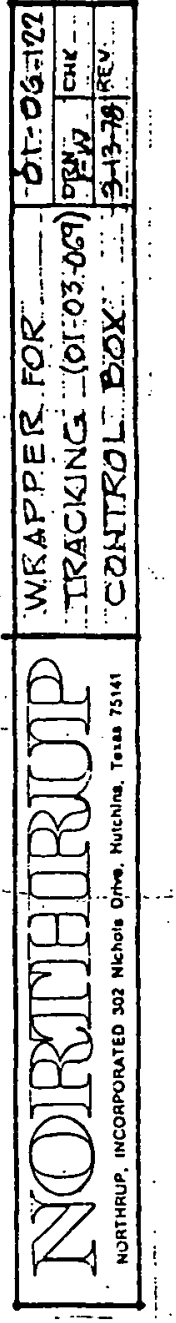

$01-06-122$ 


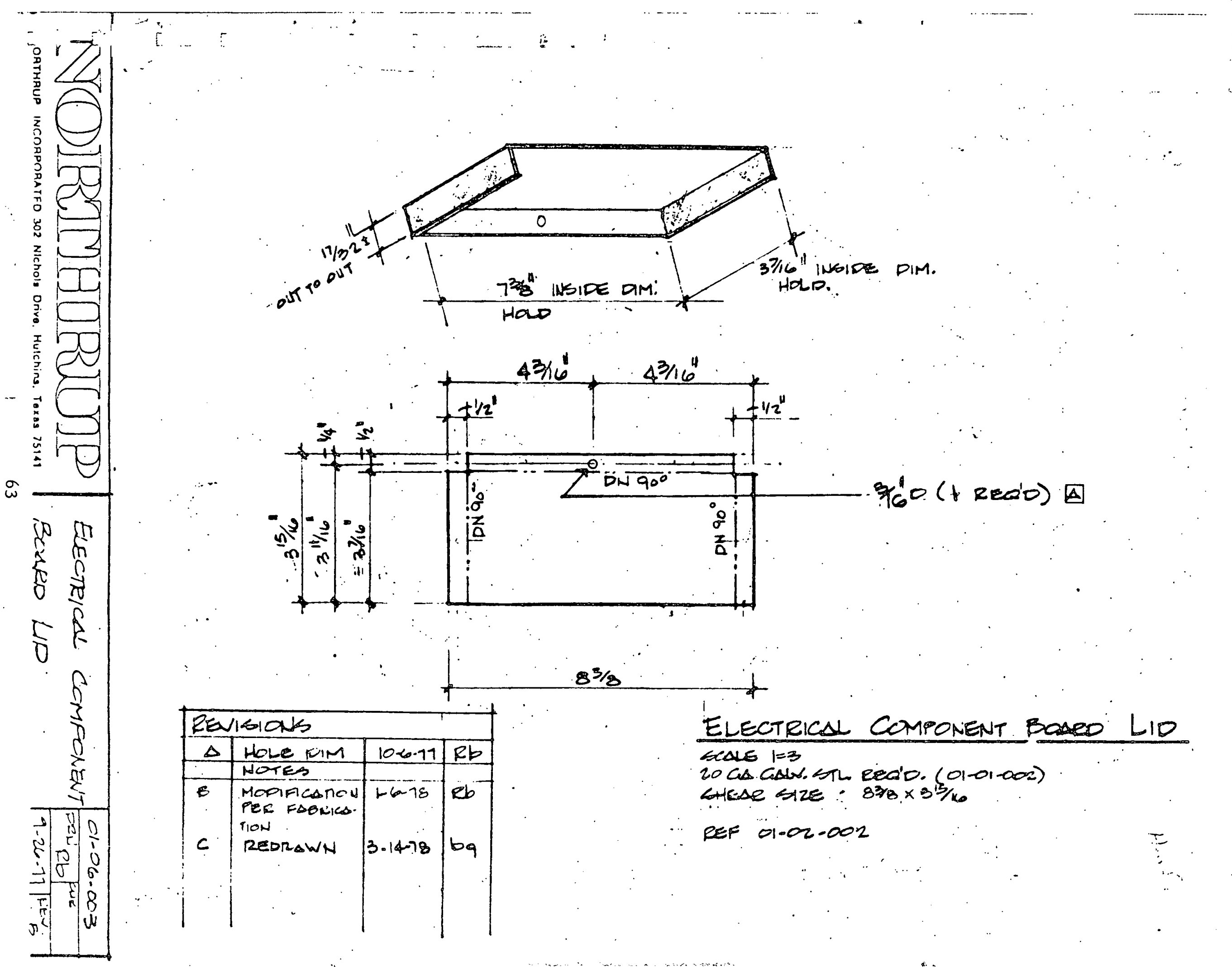



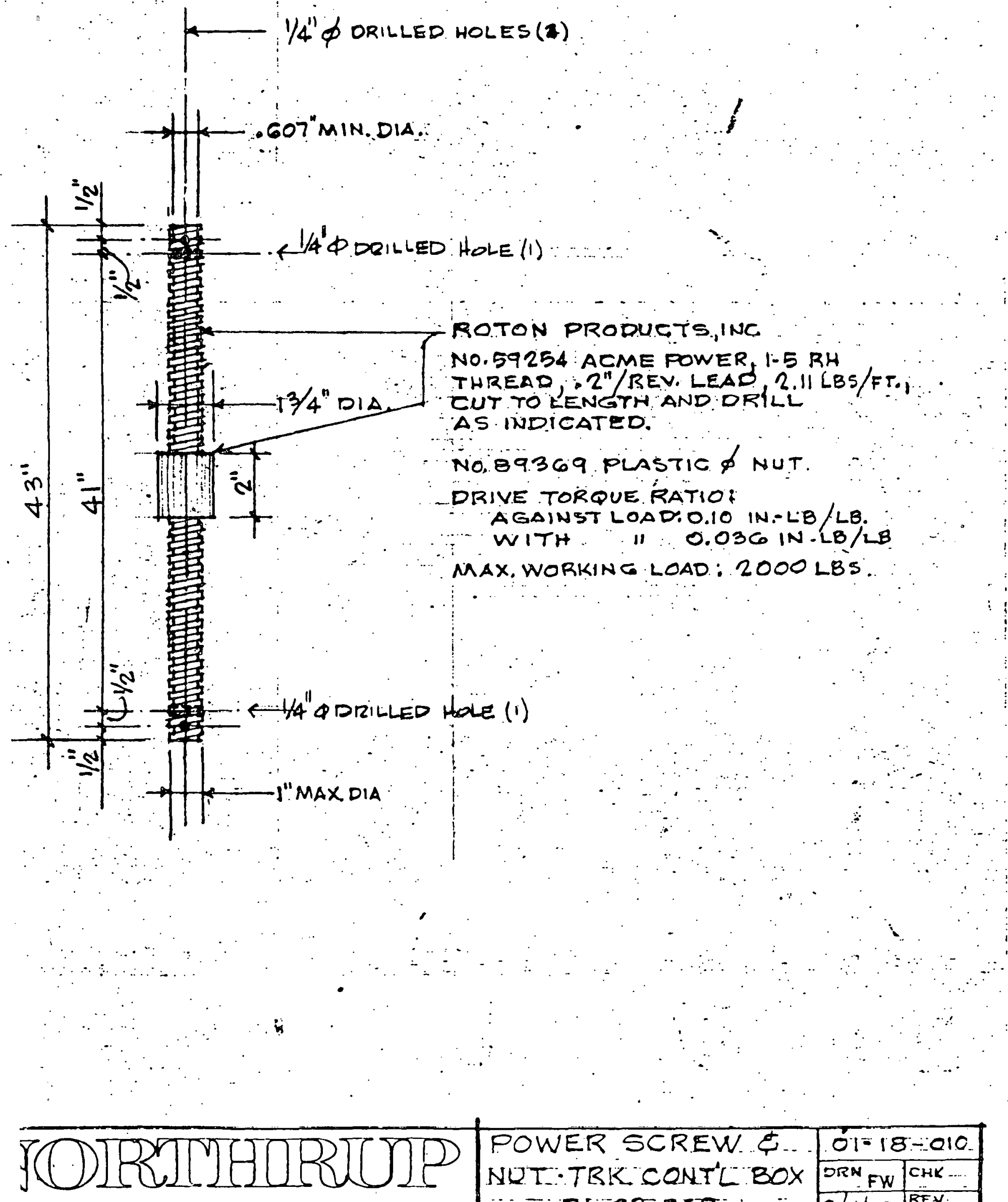

IRUP. INCORPORATED 302 Nichole Orivo. Hulchins, Taxne 75141

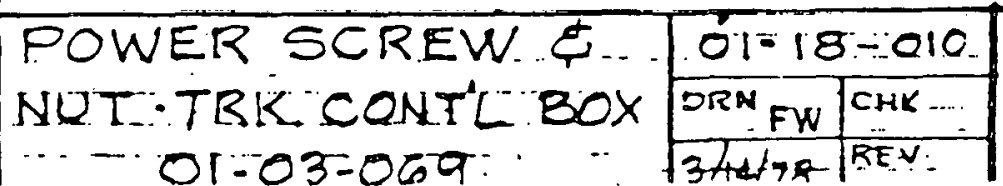




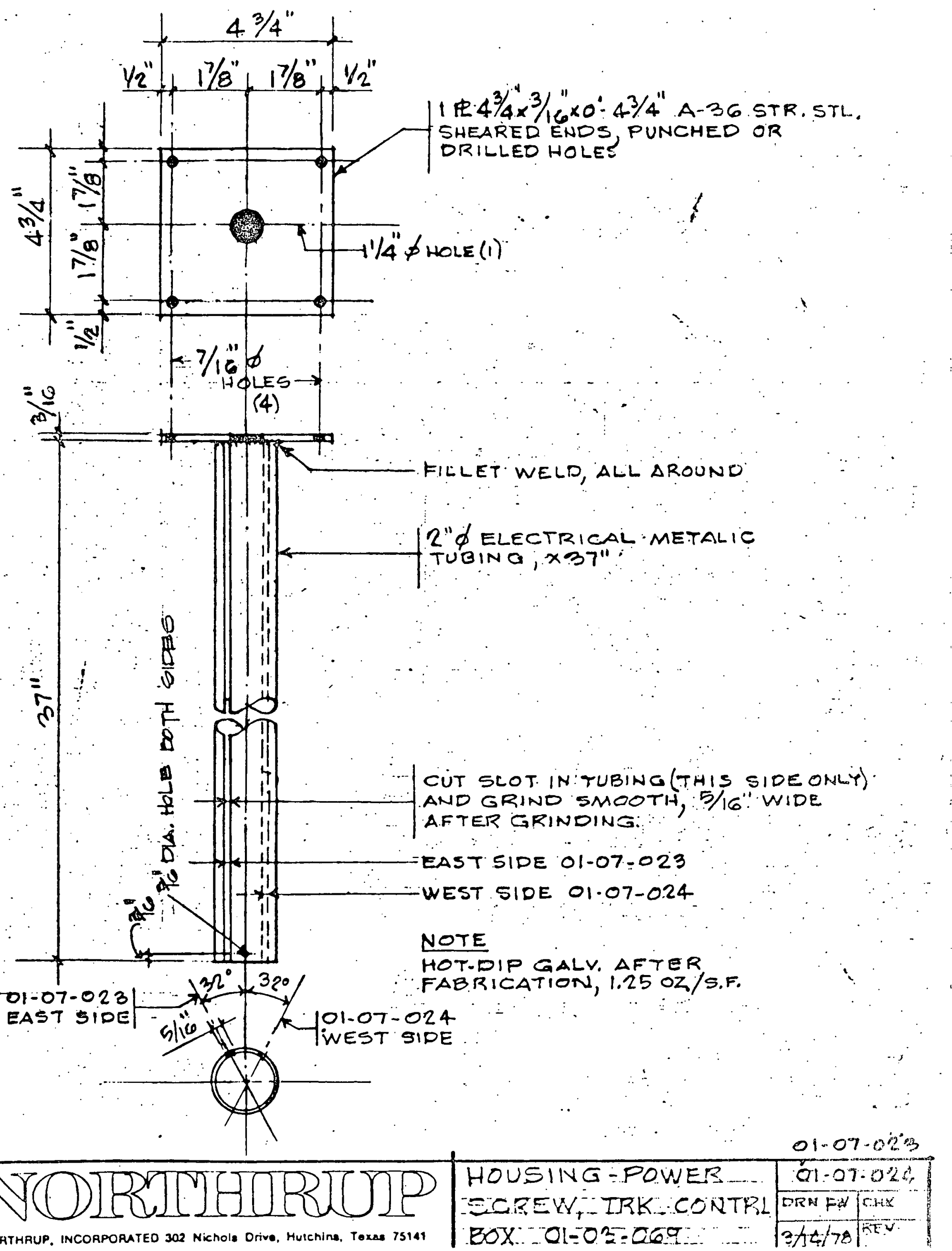



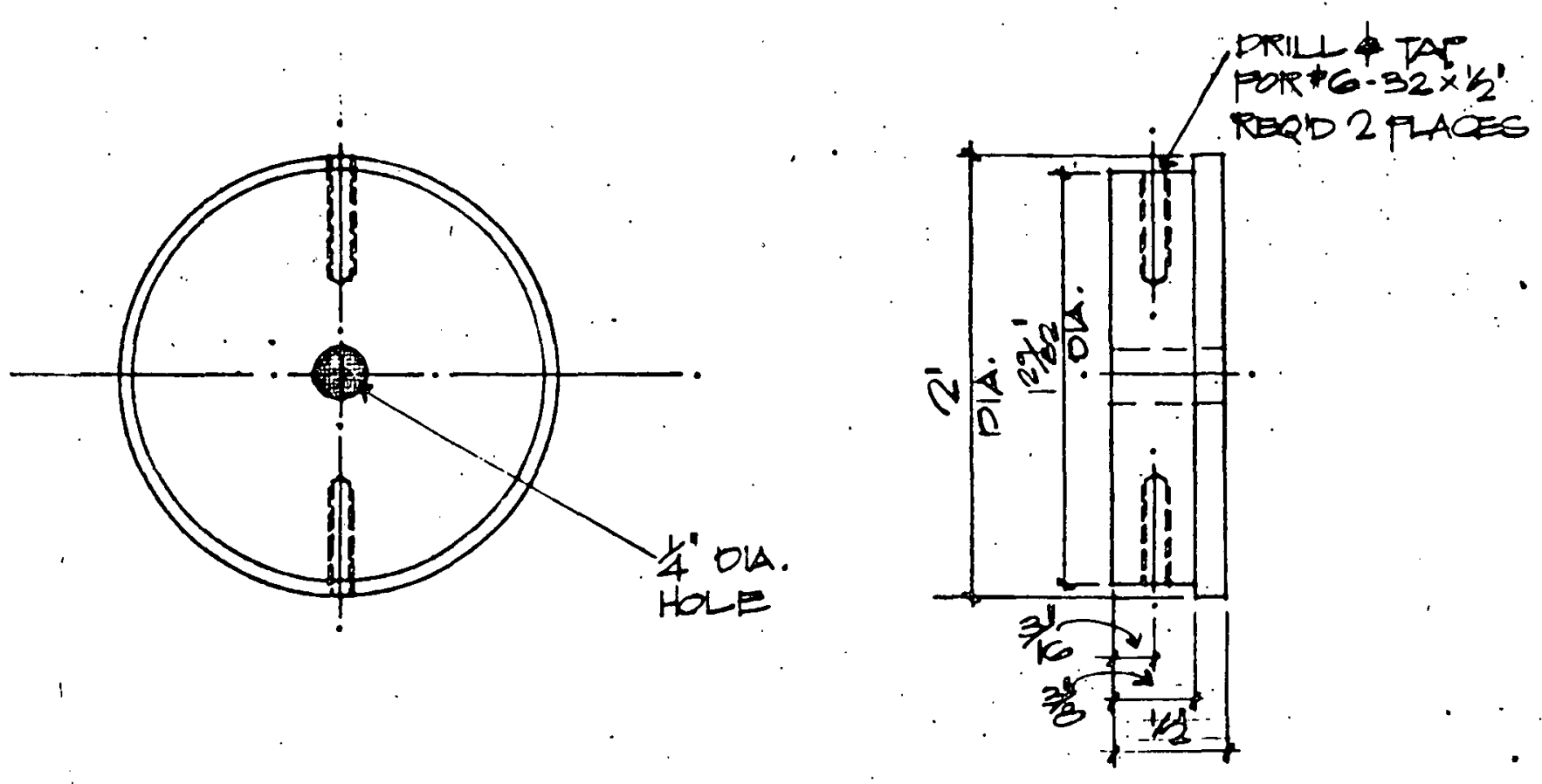

CABLE GULIDE

$$
\begin{aligned}
& \text { "PELRIN" PLASTIC (DI.PONT) "500 } \\
& \text { 2EA. REQID PET ASS'Y }
\end{aligned}
$$

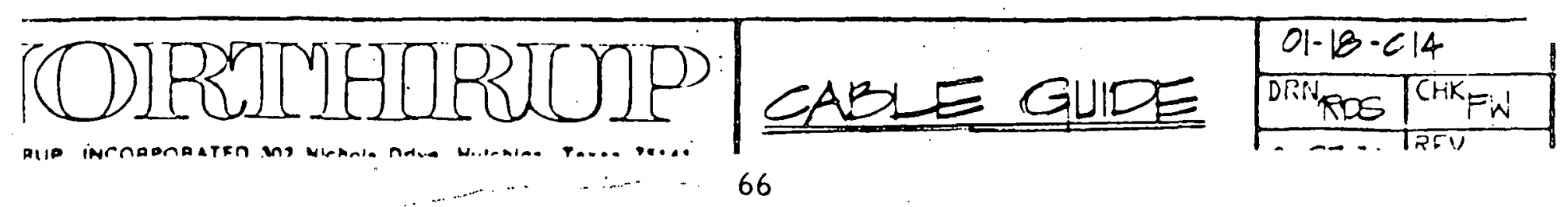




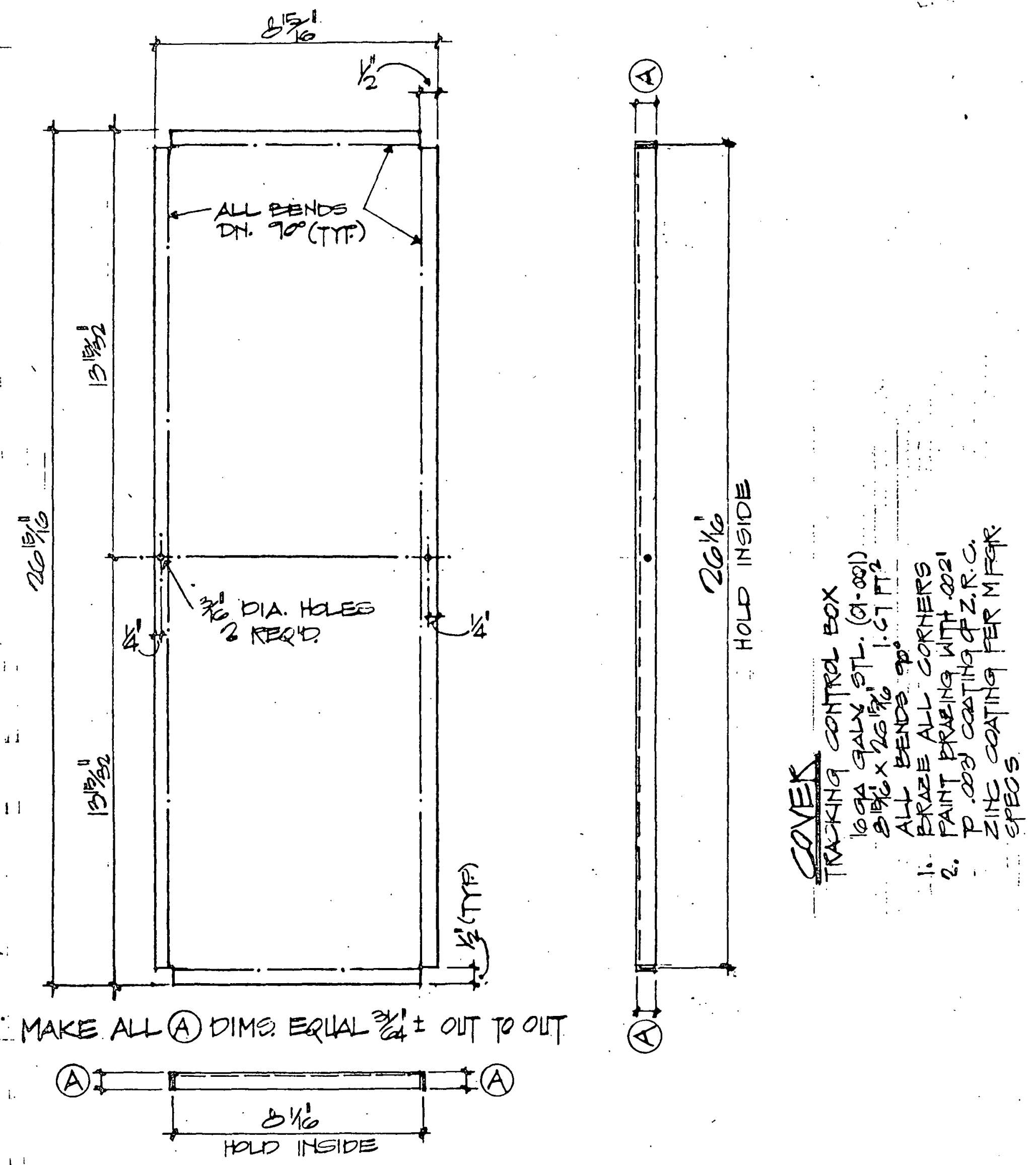

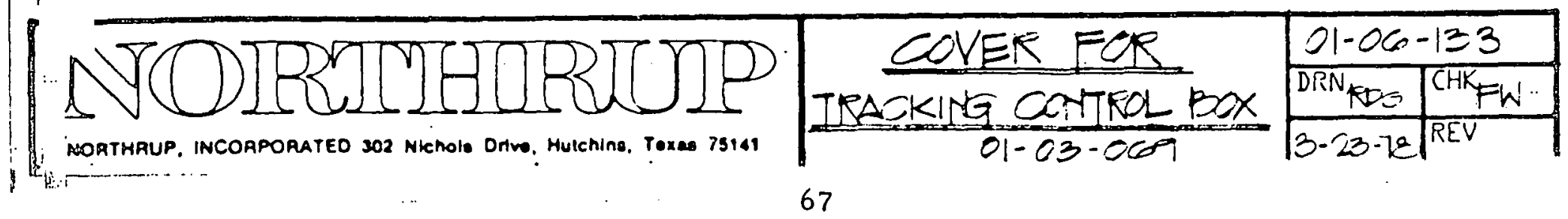



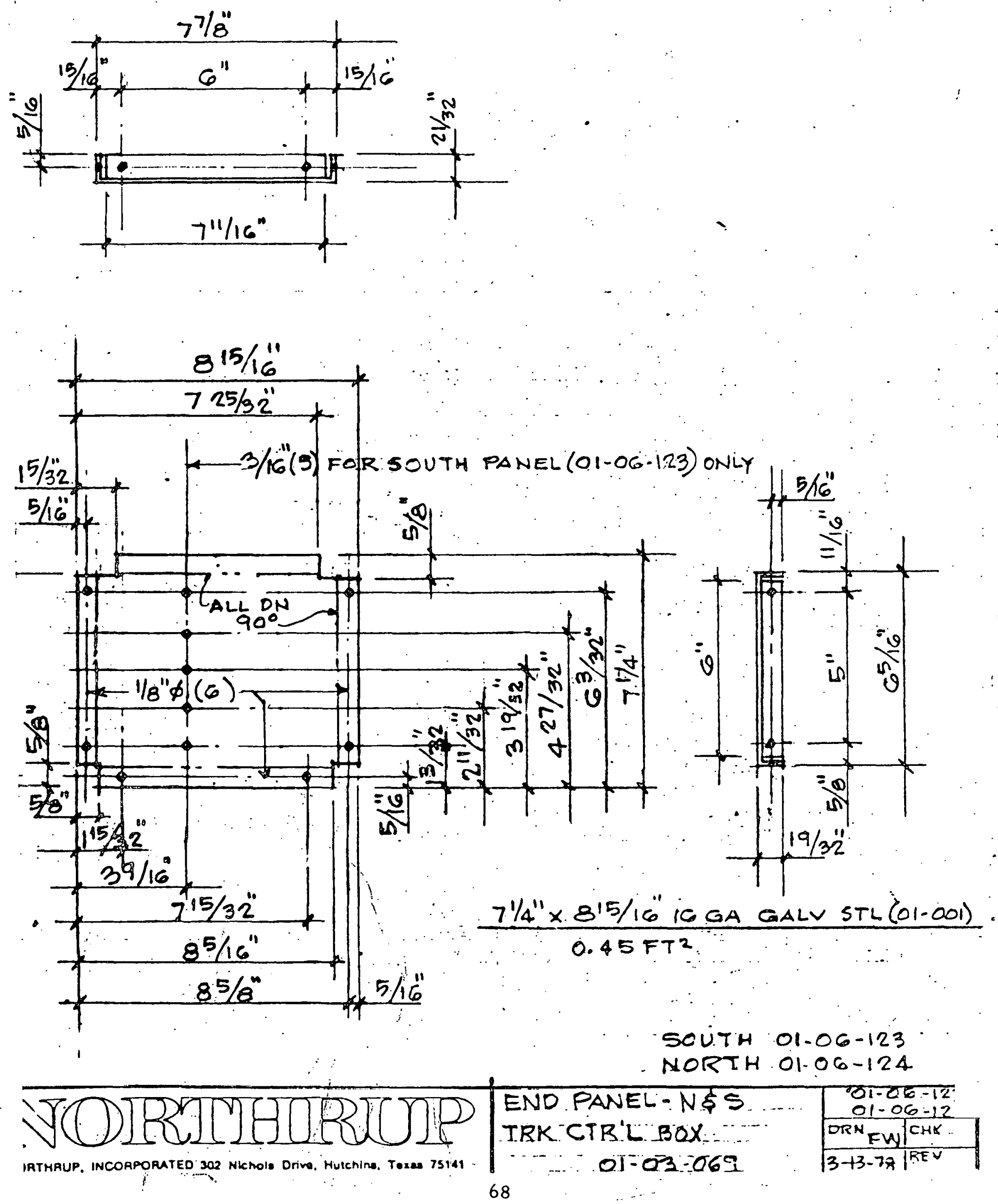

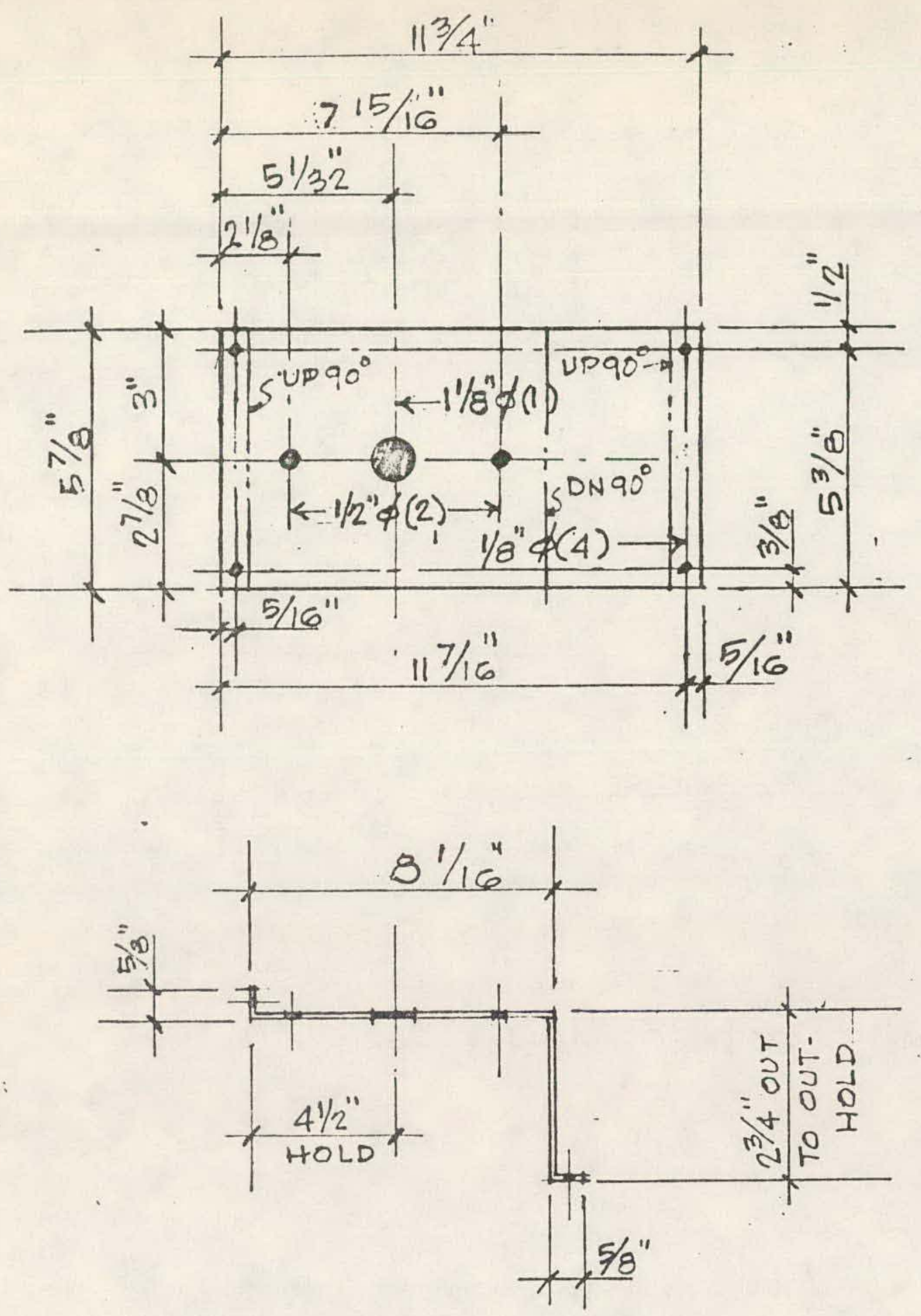

$$
\frac{113 / 4^{\prime \prime} \times 57 / 8^{n} 16 \text { GA, GALV STL }(01-001)}{0.48 \mathrm{FT}^{2}}
$$

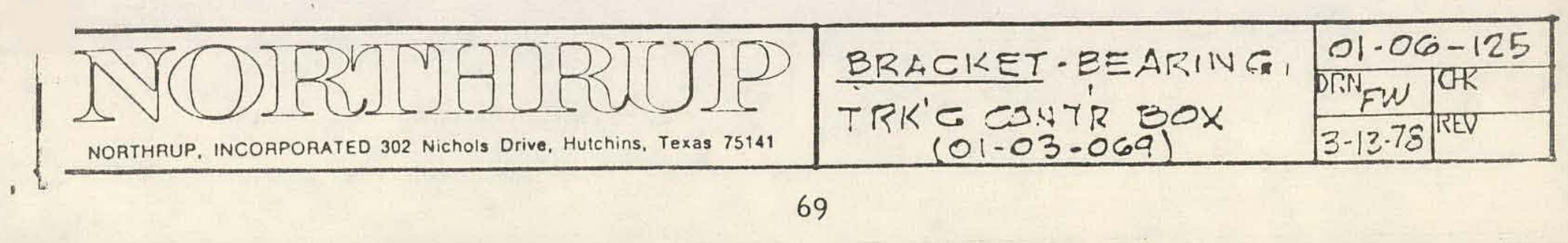


Set Screw Type

Normal Duty
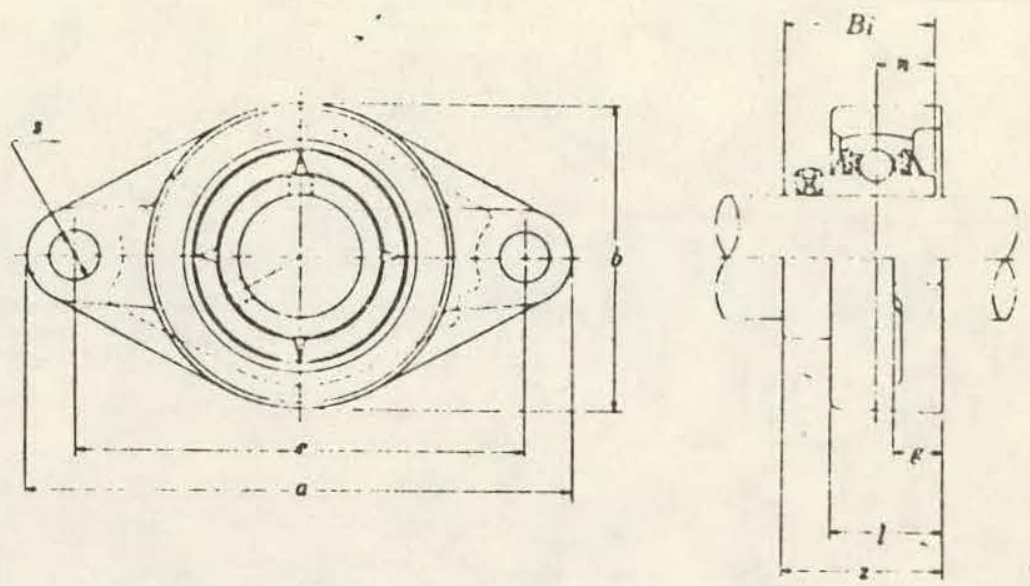

\begin{tabular}{|c|c|c|c|c|c|c|c|c|c|c|c|c|c|c|}
\hline $\begin{array}{l}\text { Shaft } \\
\text { Dia. } \\
\text { in }\end{array}$ & $\begin{array}{c}\text { Complete } \\
\text { Flanged Unit } \\
\text { Number }\end{array}$ & $a$ & e & $g$ & Nomina & Nime: & siuns in. & 2 & $B i$ & $n$ & $\begin{array}{l}\text { Bearing } \\
\text { Niumber }\end{array}$ & $\begin{array}{l}\text { Basic } \\
\text { Rating } \\
\text { Dyn. } \\
\text { C }\end{array}$ & $\begin{array}{c}\text { load } \\
\text { g lbs. } \\
\text { Static } \\
\text { Co }\end{array}$ & $\begin{array}{l}\text { Housing } \\
\text { Number }\end{array}$ \\
\hline $12:$ & UCFL $\left.209-1^{3}, G^{\prime} 6\right)$ & $.71 \%$ & 5.': & $\because$. & $11 / 2$ & 57 & $4 \%$ & 2.06 & 1937 & $.748 !$ & UC $209-1 \%$ & 5650 & 4000 & FL209G 6) \\
\hline
\end{tabular}

\section{Flanged Units Cast Housing (Two Bolt)}

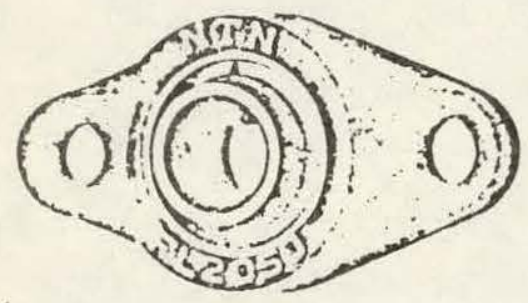

UCFL
Set Screw

UCFL 2 (for light load)

The bearing housing, each side thereof being rhombic, is to be secured to the fraine with two bolts. Its form allowing side by side mounting in a narrow space, this type is recommended where a limit is set on the mounting pitch.

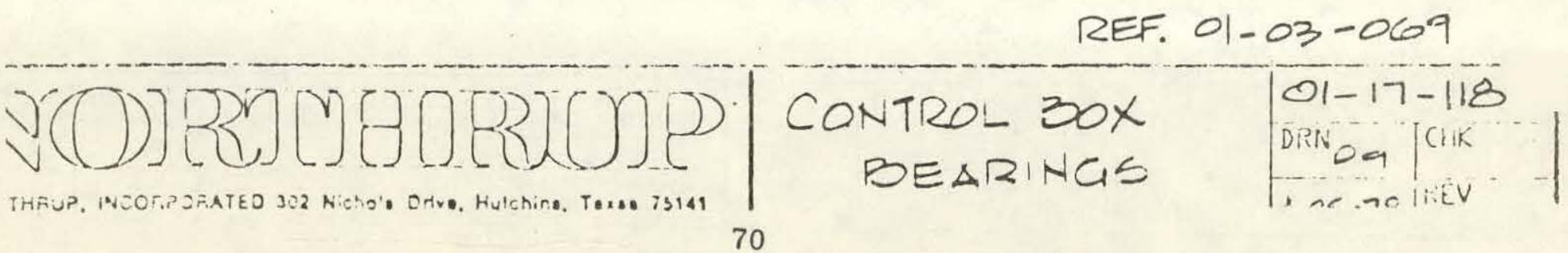




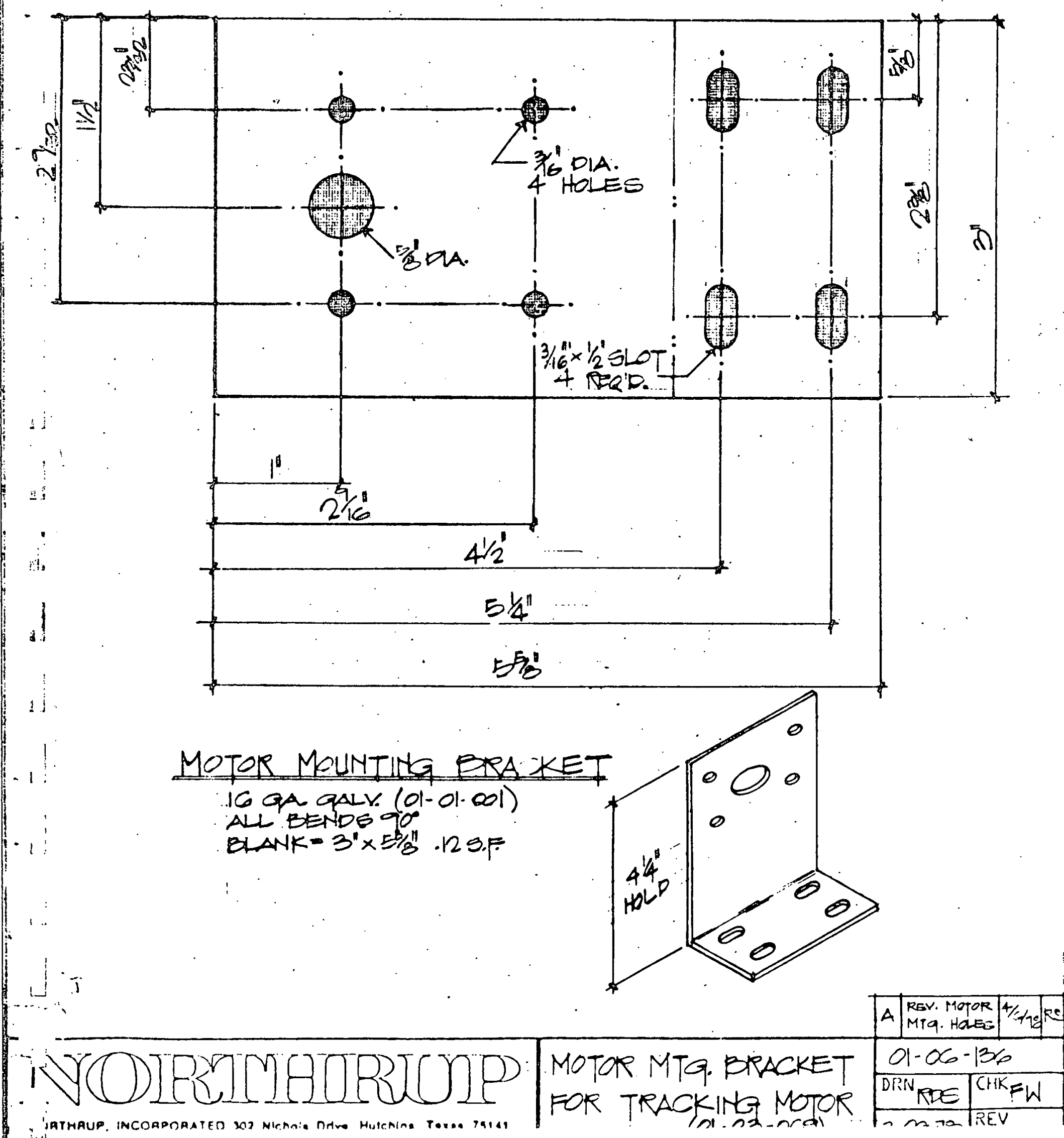



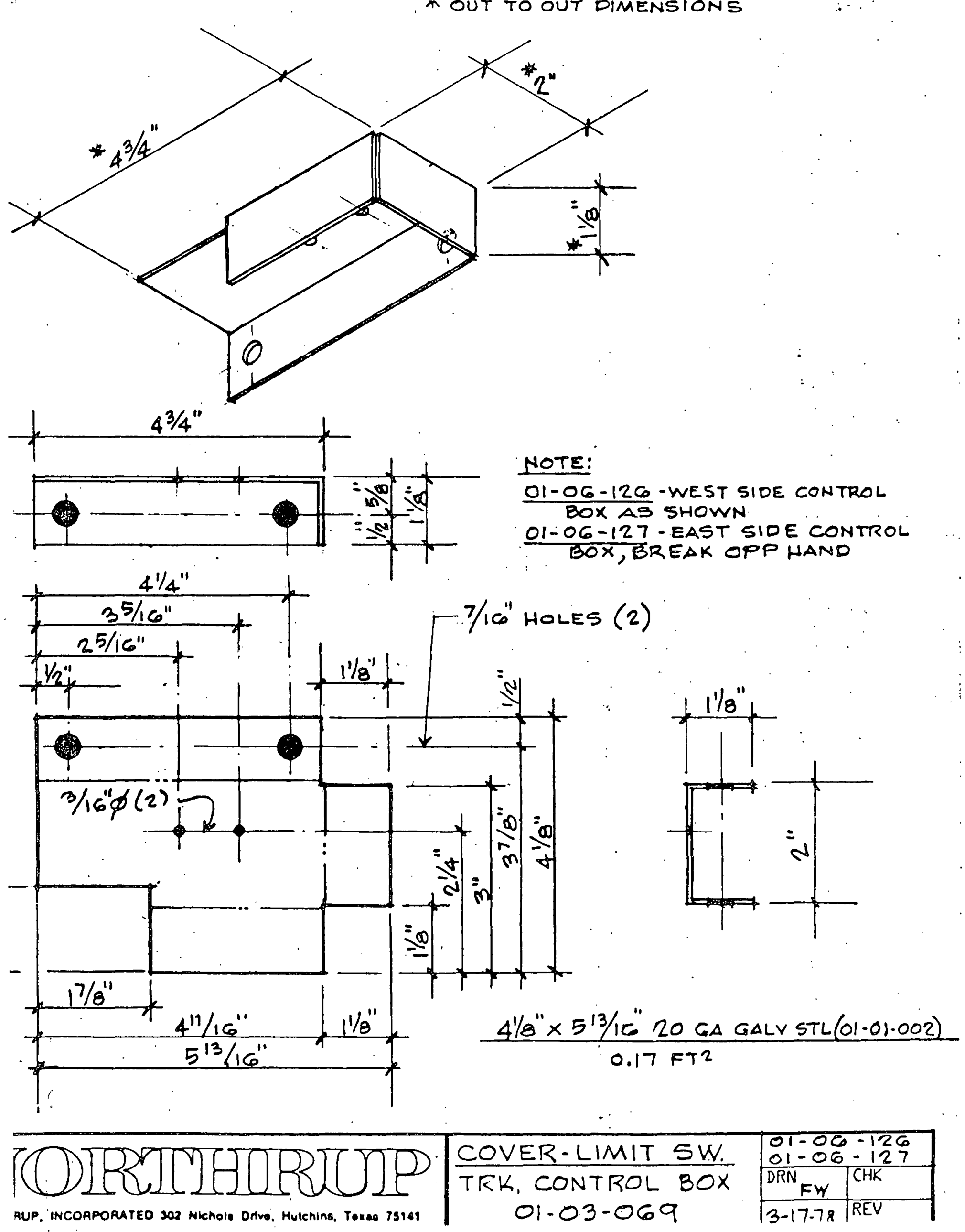

72 


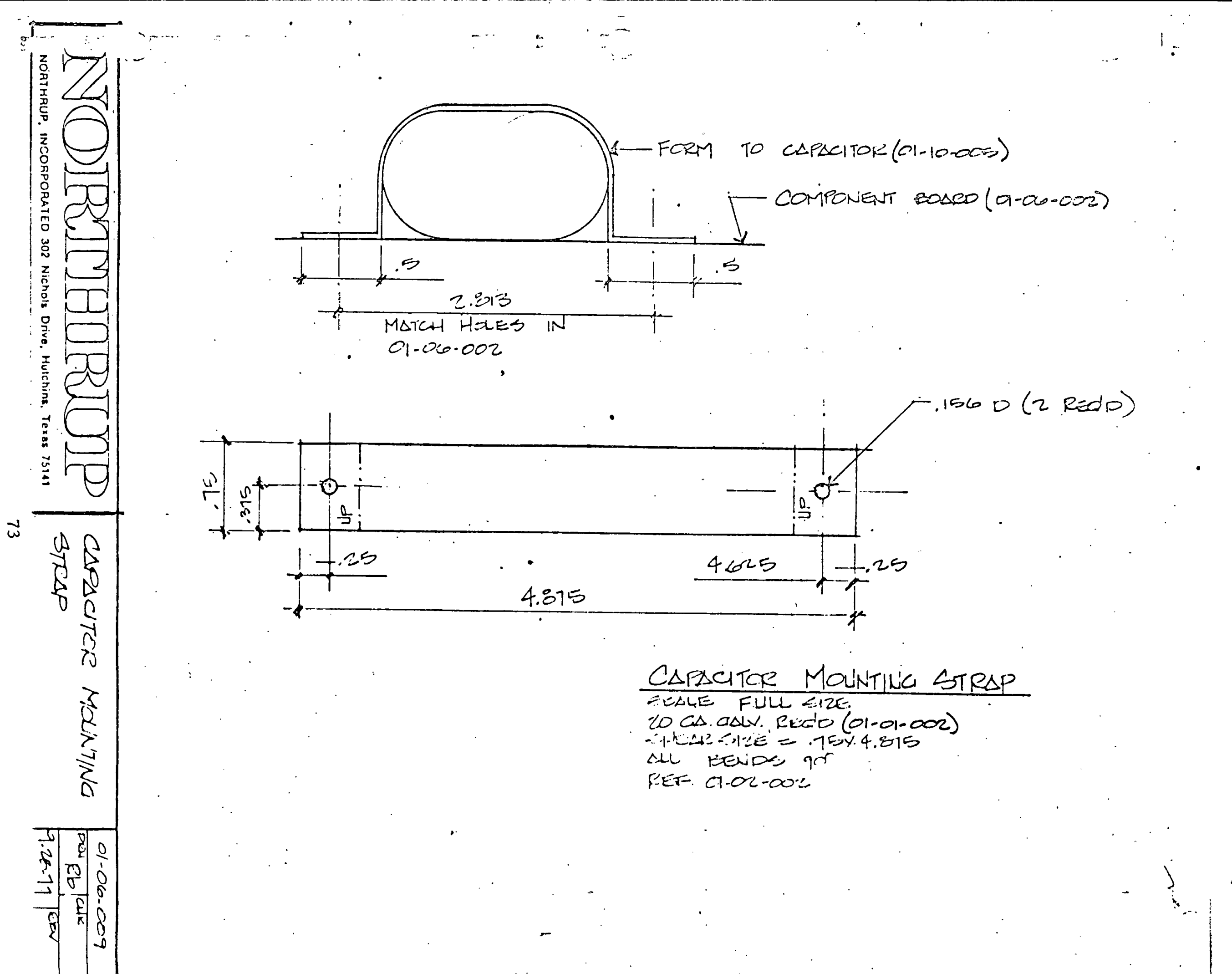




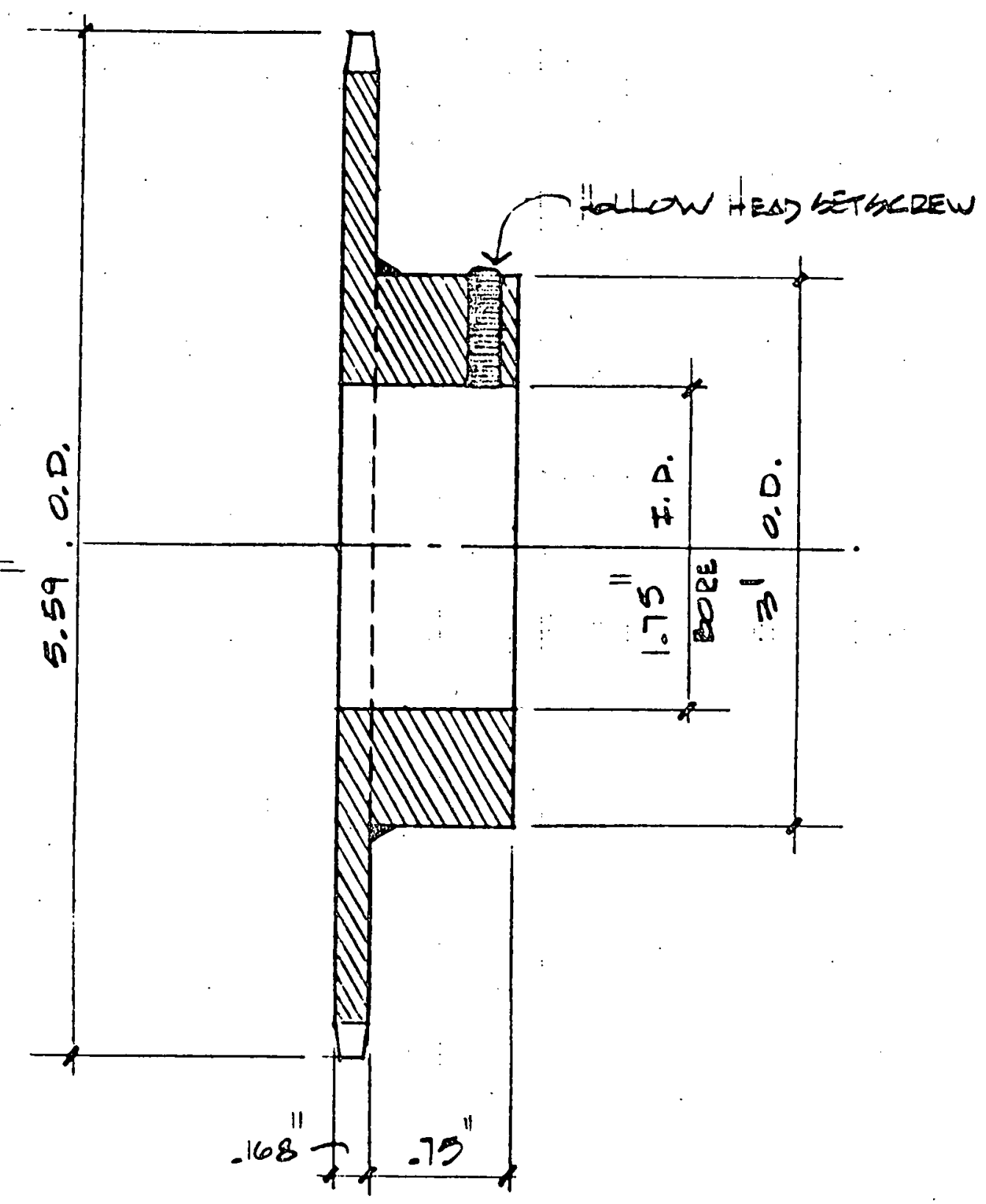

MATERIAL:

DROWNING STEE TYTE "B" \#35B45 13/4"BORE:,45 TEETH, SPROCKET.W/DET SCREW. TO DE ULED W/ Z5 $\triangle$ MERICAN OTANDARO ROLLER CHAIN. REF. O1-03-069

CONTROL DOX JRIVE EPRXLLT 


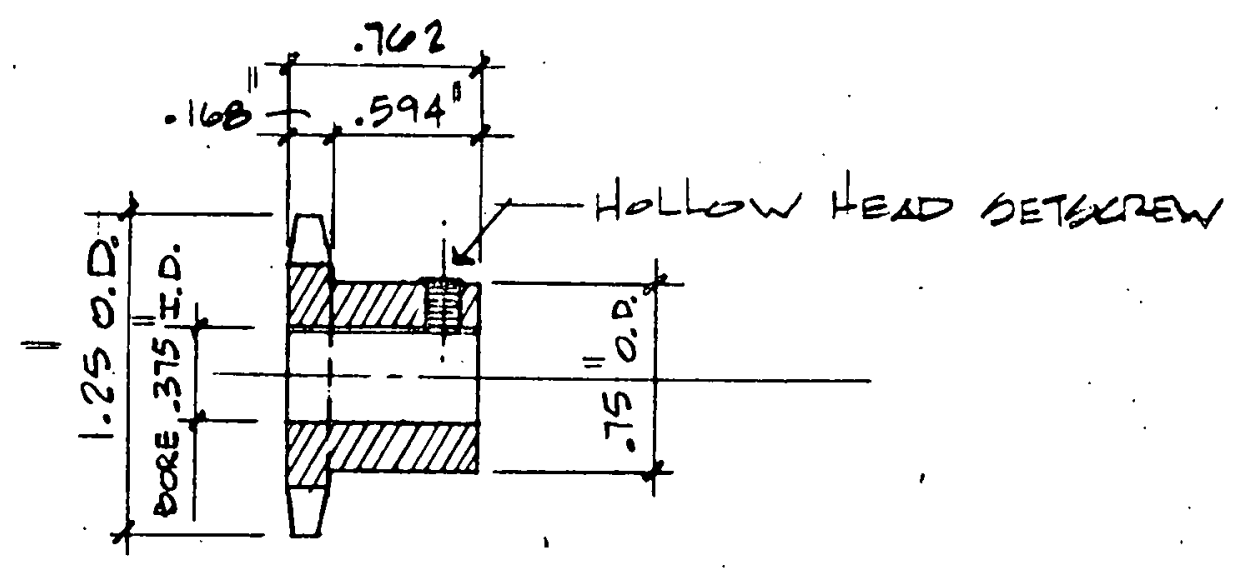

DECTON

MATERIAL

BROWNINC \# 399 PTEEL SIHGLE DTRAND SPROKKET W/9 TEETH AHD 3/B"Q RORE. $\triangle L$ LOO W' BET SCREW. TOBEUSEO $W /$ WI $\triangle$ MERIES $N$ STAHDARD ROLLER CHAIN. REF. 01-03-069.

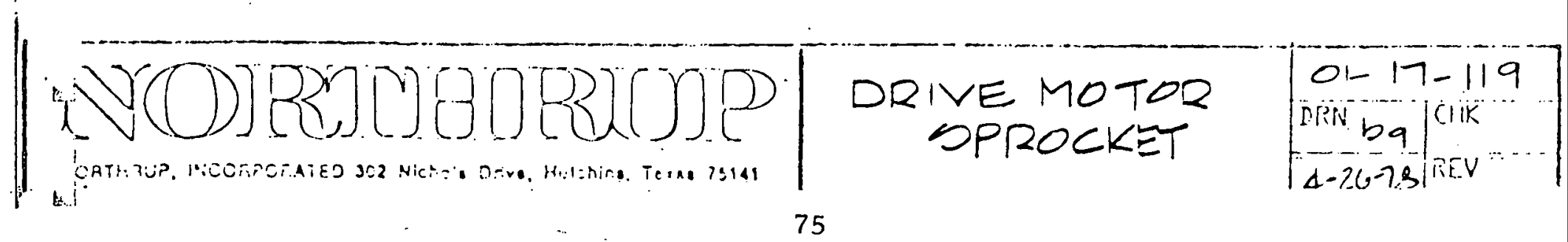




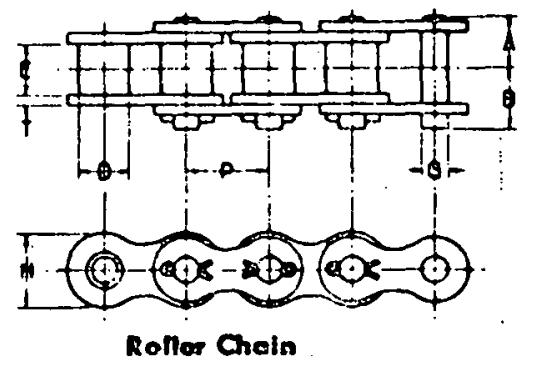

SINGLE STRAND CHAIN

6

\begin{tabular}{|c|c|c|c|c|c|c|c|c|c|c|}
\hline \multirow{3}{*}{$\begin{array}{l}\text { Mooin } \\
\text { pirch } \\
\text { Plo }\end{array}$} & \multirow{3}{*}{$\begin{array}{l}\text { CAola } \\
\text { No. }\end{array}$} & \multicolumn{2}{|c|}{ CMAIN } & \multicolumn{7}{|c|}{ DIMENSONS } \\
\hline & & \multirow{2}{*}{$\begin{array}{l}\text { Averooe } \\
\text { Ionsile } \\
\text { Sirengih } \\
\text { los. }\end{array}$} & \multirow{2}{*}{$\begin{array}{l}\text { Averogo } \\
\text { welght } \\
\text { per fi. } \\
\text { lbs. }\end{array}$} & \multicolumn{2}{|c|}{$\begin{array}{c}\text { Connecting } \\
\text { lint, }\end{array}$} & \multicolumn{2}{|c|}{ : Rollere: } & \multirow{2}{*}{$\frac{\text { Pini }}{G}$} & \multicolumn{2}{|c|}{ : Side Plotes } \\
\hline & & & & $\lambda$ & - & 0 & E & & H: & $\boldsymbol{T}$ \\
\hline
\end{tabular}

STANDARD

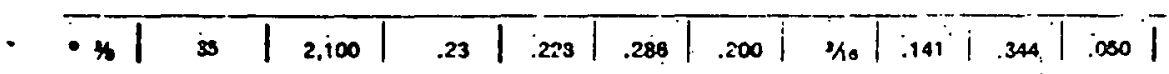

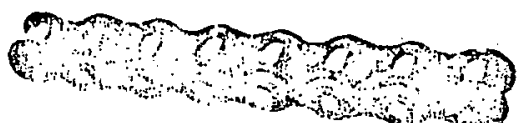

Single Strans-airetad

MATERIAL

PROUHCA ROLED.CHAN

$N^{\circ} 35$ XMERICAN STLLIDARD

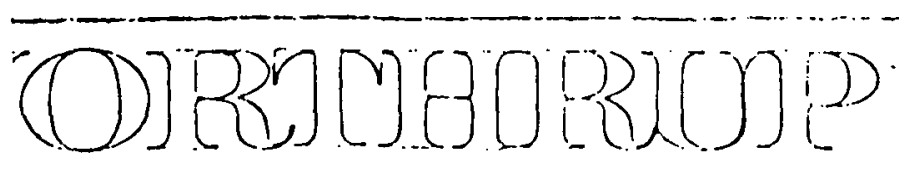

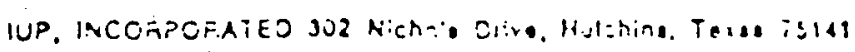

COATROL- DOX
DRIVECHAN

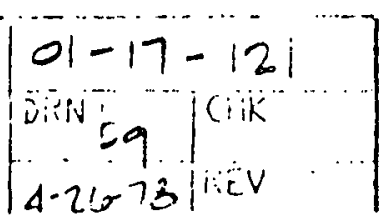


WMPHENOL Industric

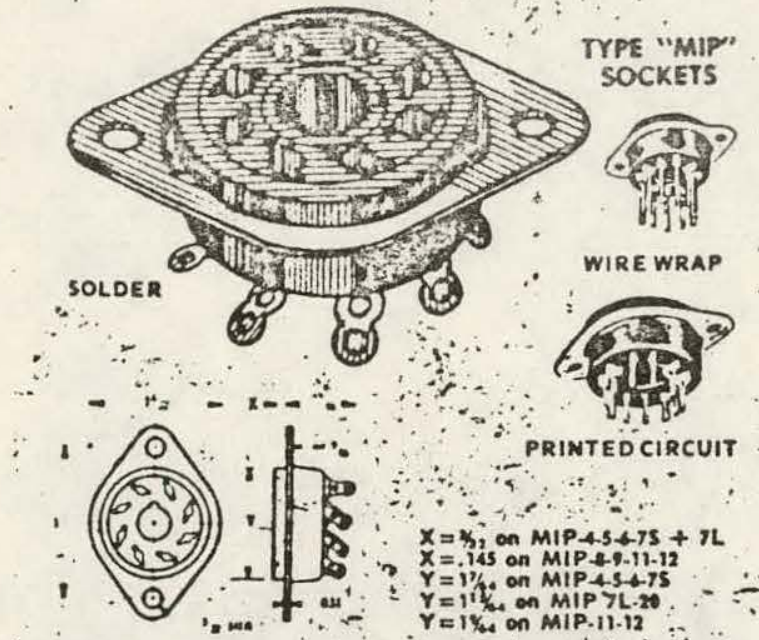

High dielectric bakellte body with molded in steel plate except (I)

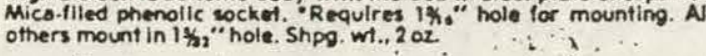

\begin{tabular}{|c|c|c|c|c|c|c|c|}
\hline $\begin{array}{l}\text { Stock } \\
\text { No. }\end{array}$ & Trpe & Prongs & 149 & 90 & 200 & $230-$ & $\begin{array}{l}1000 \\
2499\end{array}$ \\
\hline $30 \mathrm{~F} 240$ & $77 M I P_{4}$ & 4 & .26 & .22 & .193 & .151 & .135 \\
\hline 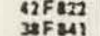 & $\begin{array}{l}\text { TIMIPST } \\
\text { TIMIPS }\end{array}$ & s & ?7? & .24 & 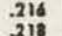 & .167 & 144" \\
\hline BAFBA & TIMIPS & & .31 & .26 & .235 & .152 & 167 \\
\hline $38 \mathrm{~F} 243$ & TIM:P7S & & .24 & .20 & .252 & 195 & .174 \\
\hline $38 \mathrm{~F} 245$ & $77 \mathrm{MIP8}$ & Octal & .26 & .2 & .196 & .152 & .136 \\
\hline 345246 & 28 & Octal I & .27 & .24 & .220 & .170 & .150 \\
\hline $38 F 47$ & 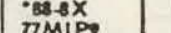 & Loktal & .44 & ב & . .330 & .254 & .226 \\
\hline $\begin{array}{l}2 \mathrm{AF} 240 \\
3 \mathrm{~B} F \mathrm{BO}\end{array}$ & $\begin{array}{l}\text { TMMIP } \\
\text { TMIPII }\end{array}$ & iा & .30 & .23 & .228 & .1735 & $\begin{array}{l}.156 \\
.160\end{array}$ \\
\hline $39 F 1244$ & TIMIPIIT & II & .40 & .24 & .302 & .239 & .207 \\
\hline $\begin{array}{l}30 F=30 \\
30 F 109\end{array}$ & $\begin{array}{l}77 M 1 P 12 \\
\text { חMIPZO }\end{array}$ & 12 & .70 & $\begin{array}{l}.30 \\
.50\end{array}$ & .269 & $\begin{array}{l}.203 \\
.410\end{array}$ & .1863 \\
\hline \multicolumn{8}{|c|}{ PRINTED CIRCUIT TYPES } \\
\hline $\begin{array}{c}\text { Stock } \\
\text { No. }\end{array}$ & Type & Prongs & $1-49$ & 30. & 250- & son. & $\begin{array}{l}10000 \\
2499\end{array}$ \\
\hline & TMM1PBW-0123 & 8 & .35 & .7 & .204 & .205 & .102 \\
\hline $42 \mathrm{~F} 28$ & TM1P9W-0125 & is & .49 & . 3 & . 295 & .220 & .205 \\
\hline $42 F=27$ & $77 M 1 P 11 W-0125$ & 11 & 1.42 & & .317 & .246 & .219 \\
\hline \multicolumn{8}{|c|}{ WIRE WRAP TYPES } \\
\hline $42 F 828$ & $77 M I P a W-0500$ & 10 & $|x|$ & .2 & .295 & .228 & .200 \\
\hline $42 \mathrm{~F} 320$ & TMIPIIW.0500 & iI & .47 & .4. & .31257 & 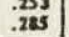 & .253. \\
\hline
\end{tabular}

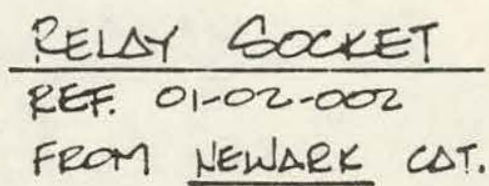


WIRE SET

ELECTRICAL. COMPONENT ASSENBLY

RF.TCH ASSOCIATES, INC.

P. 0. Box 73, Plano, Texas 75074

(214) $424-7904$

October 27,1977 Rev. 1(11/11/77) Rev. 2(11/18/77), Rev. 3(12/12/77)Rb Rev. $4(3 / 38 / 78)$ FW.

Mode1 10062

Cut Wire List Racker Electronics Sub Assembly

\begin{tabular}{|c|c|c|c|c|c|c|}
\hline $\begin{aligned} \text { WIRE } & \text { NO. } \\
1 & \end{aligned}$ & $\begin{array}{c}\text { QUANT ITY } \\
1\end{array}$ & $\begin{array}{l}\text { COLOR } \\
\text { BIack. }\end{array}$ & $\begin{array}{l}\text { LENGTH } \\
10^{\prime \prime}\end{array}$ & REMARKS & $\begin{array}{l}\text { FROM } \\
\text { Inside }\end{array}$ & $\begin{array}{l}\text { To } \\
\text { Outside } 120 \mathrm{~V}\end{array}$ \\
\hline 2 & 1 & B 1 a ck & $9^{\prime \prime}$ & & $F 1 / 2$ & $J 2 / 1$ \\
\hline 12 & 1 & Black & $26^{\prime \prime}$ & & $\mathrm{j} 1 / 1$ & $\begin{array}{l}\text { Outside } \\
\text { Solar Sensor }\end{array}$ \\
\hline 22 & 1 & Black & $5^{\prime \prime}$ & & $\mathrm{J} 2 / 3$ & $\mathrm{~J} 3 / 1$ \\
\hline 8 & 1 & B lack & $15^{\prime \prime}$ & & TP 1 & Outside (motor) \\
\hline 5 & 1 & Blue & $10^{\prime \prime}$ & & Inside & Outside $120 \mathrm{~V}$ \\
\hline 13 & 1 & Orange & $26 "$ & & $\mathrm{~J} 1 / 2$ & $\begin{array}{l}\text { Outside } \\
\text { Solar Sensor }\end{array}$ \\
\hline 14 & 1 & Blue & $26^{\prime \prime}$ & & $\mathrm{J} 1 / 3$ & $\begin{array}{l}\text { Outside } \\
\text { Solar Sensor }\end{array}$ \\
\hline 18 & 1 & Blue & $18^{\prime \prime}$ & $\begin{array}{l}\text { Flag } 1 \\
\text { End }\end{array}$ & $\mathrm{J} 2 / 2$ & $\begin{array}{l}\text { Outside } \\
\text { Limit Switch }\end{array}$ \\
\hline 21 & 1 & Blue & $5 "$ & & $\mathrm{~J} 2 / 7$ & $\mathrm{~J} 1 / 12$ \\
\hline 15 & 1 & Green & $5 "$ & & $\mathrm{~J} 1 / 4$ & JP 2 \\
\hline 16 & 1 & Green & $21^{\prime \prime}$ & $\begin{array}{l}\text { Flag } 1 \\
\text { End }\end{array}$ & TP 2 & $\begin{array}{l}\text { Outside } \\
\text { Limit Switch }\end{array}$ \\
\hline 19 & 1 & Red & $21 "$. & $\begin{array}{l}\text { Flag } 1 \\
\text { End }\end{array}$ & $J 3 / 2$ & $\begin{array}{l}\text { Outside } \\
\text { Limit Switch }\end{array}$ \\
\hline 20 & 1 & Red & $5 "$ & & $\mathrm{~J} 3 / 7$ & $\mathrm{~J} 1 / 6$ \\
\hline 23 & 1 & Yel1 ow & $15^{\prime \prime}$ & $\begin{array}{l}\text { Flag } 1 \\
\text { End }\end{array}$ & $\mathrm{C} 1 / 1$ & Outside (motor) \\
\hline 24 & 1 & Yellow & $8^{\prime \prime}$ & $\begin{array}{l}\text { Flag } 1 \\
\text { End }\end{array}$ & $\mathrm{J} 2 / 6$ & $\mathrm{C} 1 / \mathrm{I}$ \\
\hline 25 & 1 & Red & $15 "$ & $\begin{array}{l}\text { Flag } 1 \\
\text { End }\end{array}$ & $\mathrm{C} 1 / 2$ & Outside (motor) \\
\hline 26 & 1 & White & $8^{\prime \prime}$ & $\begin{array}{l}\text { Flag } 1 \\
\text { End }\end{array}$ & $\mathrm{J} 3 / 6$ & $\mathrm{C} 1 / 2$ \\
\hline 17 & 1 & Green & $18^{\prime \prime}$ & $\begin{array}{l}\text { Flag } 1 \\
\text { End }\end{array}$ & TP 2 & $\begin{array}{l}\text { Outside } \\
\text { Limit Switch }\end{array}$ \\
\hline
\end{tabular}

NOTE: All wire 18 gauge. Cut to specified length and strip ends 


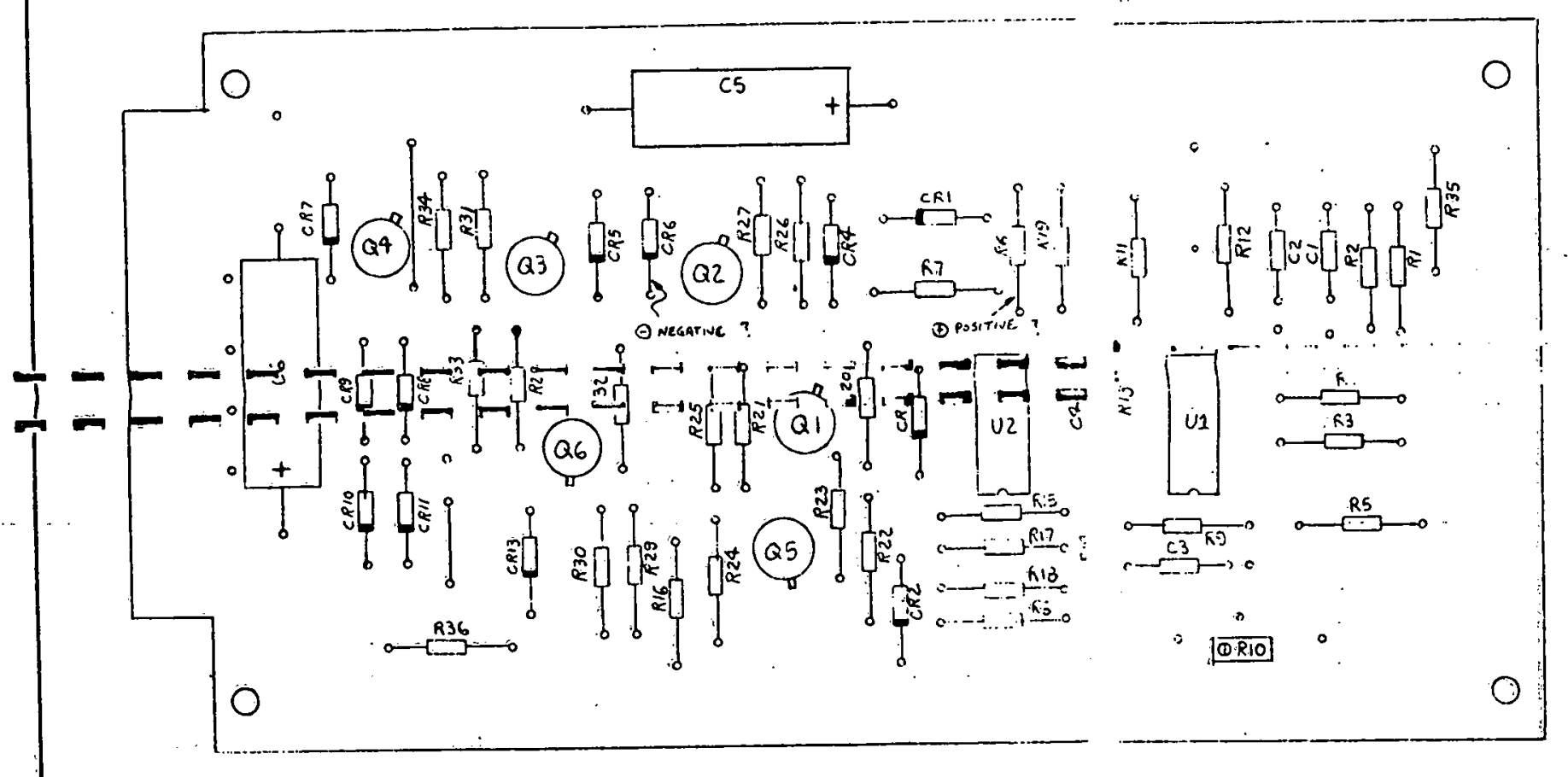

NOPRTCHORUIOP

REICH ASSOCIATES, INC Flano, TX.

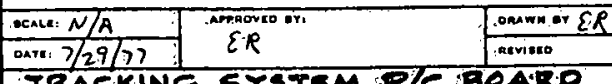
TRACKINC SYSTEM PI
ASSEMBLY 


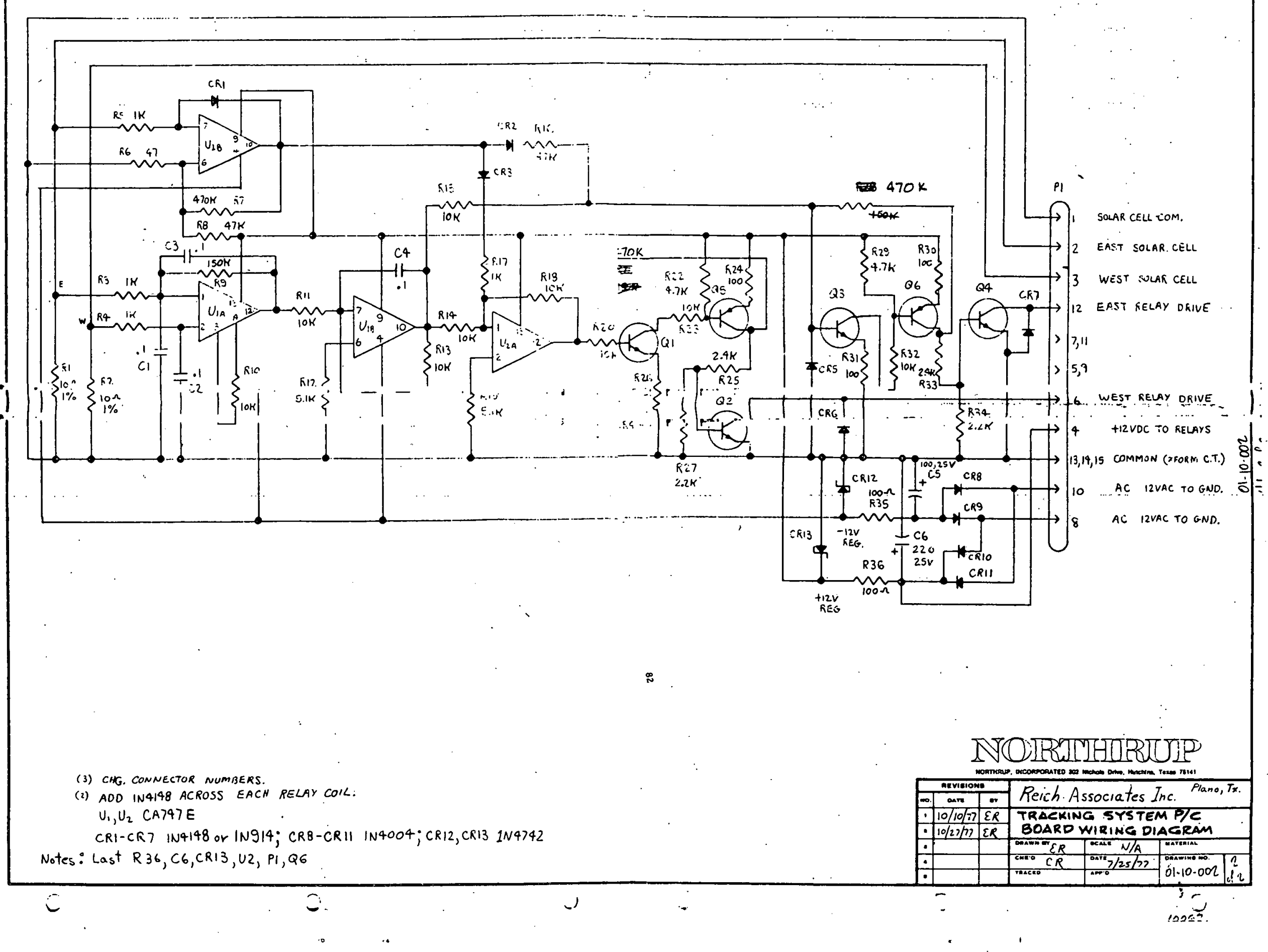




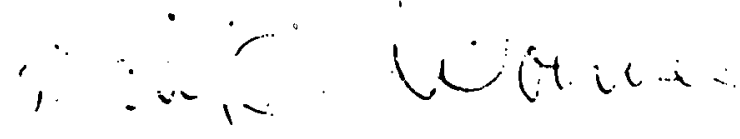

\section{WIRING DIAGRAM}

Connection for Clockwise (CW) or Counterclockwise (CCW) rotation facing output shaft; connect 115 VAC, $60 \mathrm{~Hz}$ power sup. ply as illustrated.
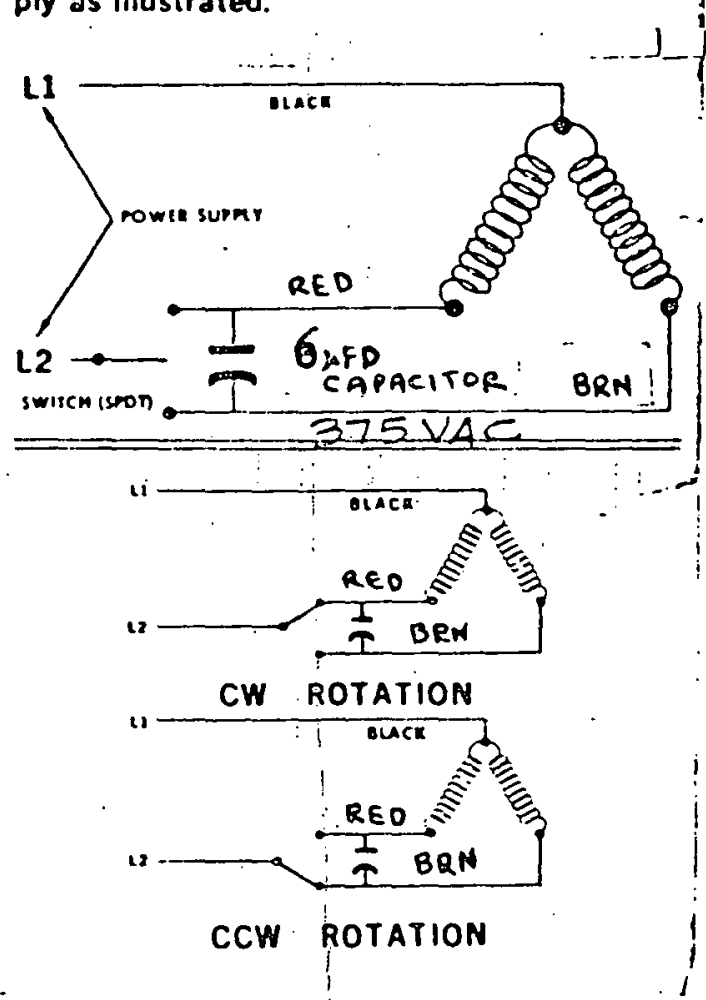


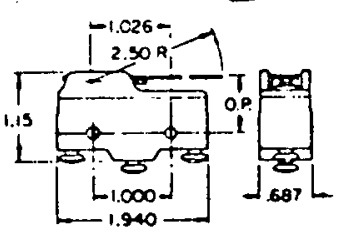

STYLE 22

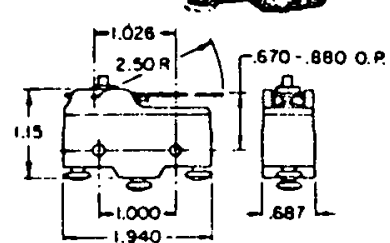

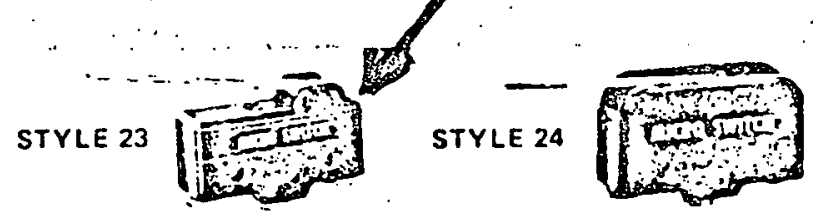
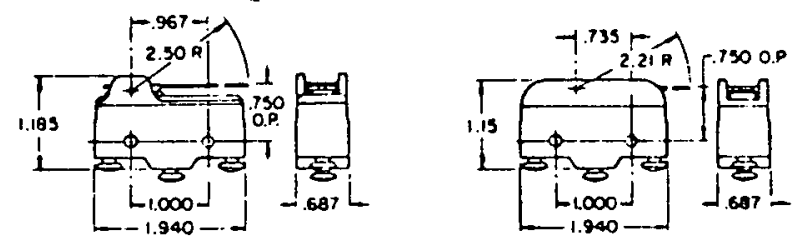

\section{ORDER GUIDE}

ROLLER LEVER
Characteristics: O.F. - Operating Force:

R.F. - Release Force: P.T. - Pretravel:

O.T. - Overtravel: D.T. - Differential Travel:
O.P. - Operating Position: F.P. - Free Position

\begin{tabular}{|c|c|c|c|c|c|c|c|c|c|c|}
\hline Recommended For & $\begin{array}{l}\text { Style } \\
\text { No. }\end{array}$ & $\begin{array}{l}\text { Electrical } \\
\text { Data Rel. }\end{array}$ & Typical Catalog Listing & $\begin{array}{l}\text { O.F. max. } \\
\text { (ounces) }\end{array}$ & $\begin{array}{l}\text { R.F. min. } \\
\text { (ounces) }\end{array}$ & $\begin{array}{l}\text { P.T. max. } \\
\text { (inches) }\end{array}$ & $\begin{array}{l}\text { O.T. min. } \\
\text { (inches) }\end{array}$ & $\begin{array}{c}\text { O.T. } \\
\text { (inches) }\end{array}$ & $\begin{array}{c}0 . P . \\
\text { (inches) }\end{array}$ & $\begin{array}{l}\text { F.P. max. } \\
\text { (inches) }\end{array}$ \\
\hline
\end{tabular}

\begin{tabular}{|c|c|c|c|c|c|c|c|c|c|c|c|}
\hline $\begin{array}{l}\text { Up } 1020 \text { ampere load } \\
\text { Vhandisng }\end{array}$ & 23 & G & BA.2RV.A2 & 2.5 & 0.50 & .625 & .078 & $\frac{.109}{\max .}=$ & $\begin{array}{r}.750 \\
+.030\end{array}$ & - & \\
\hline $\begin{array}{l}\text { Up to } 25 \text { ampeie load } \\
\text { handling }\end{array}$ & 23 & $\mathrm{H}$ & $\begin{array}{l}B E \cdot 2 R V \cdot A 4 \\
\ldots \ldots\end{array}$ & 2.5 & 0.50 & .625 & .078 & $\begin{array}{l}.109 \\
.\end{array}$ & $\begin{aligned} & .750 \\
= & .030\end{aligned}$ & - & \\
\hline
\end{tabular}

Up 1025 ampere load handling

\section{LIMIT SWITCH}

REFER OI-02003

FROM MERO SWITCH CATALOA 


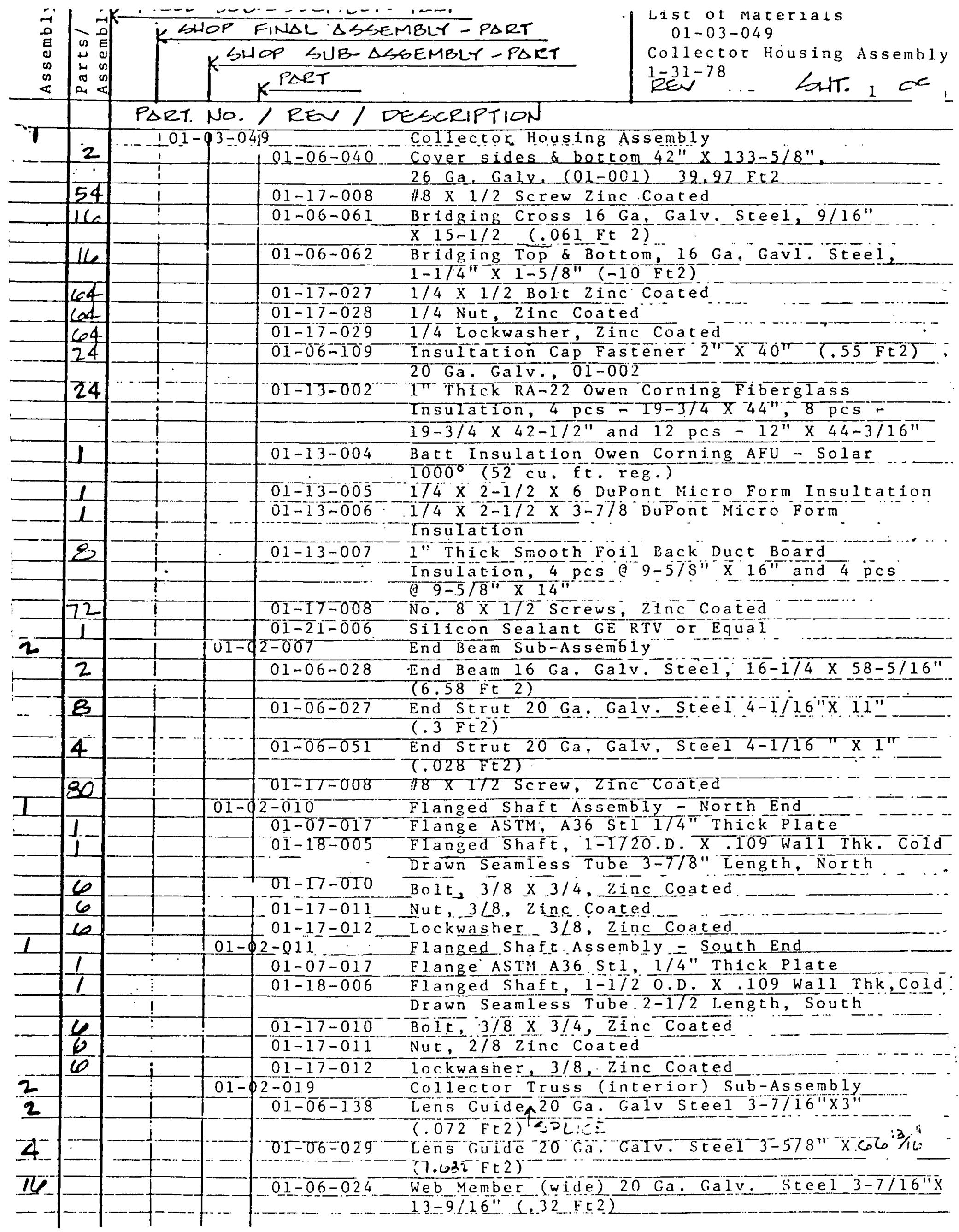




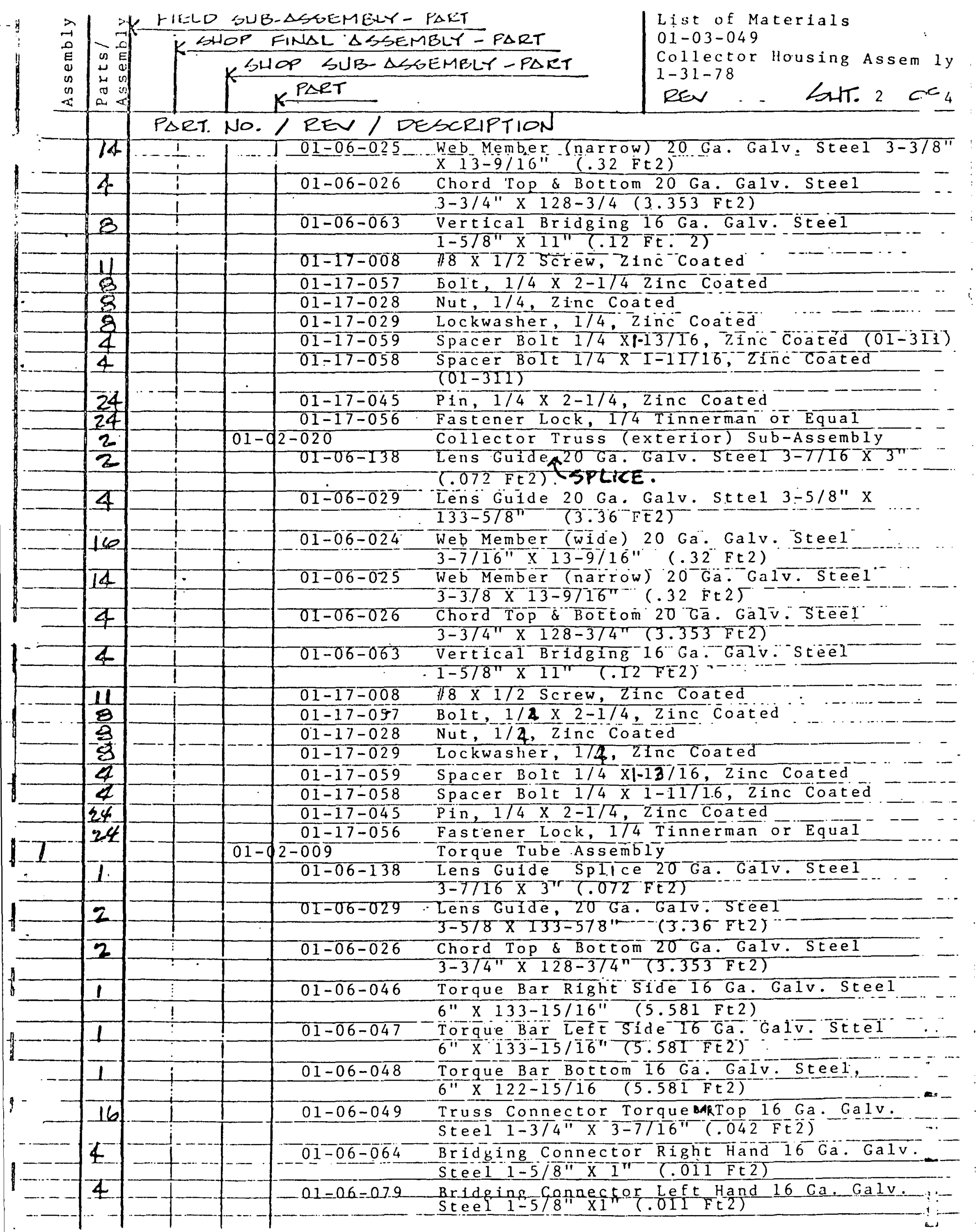




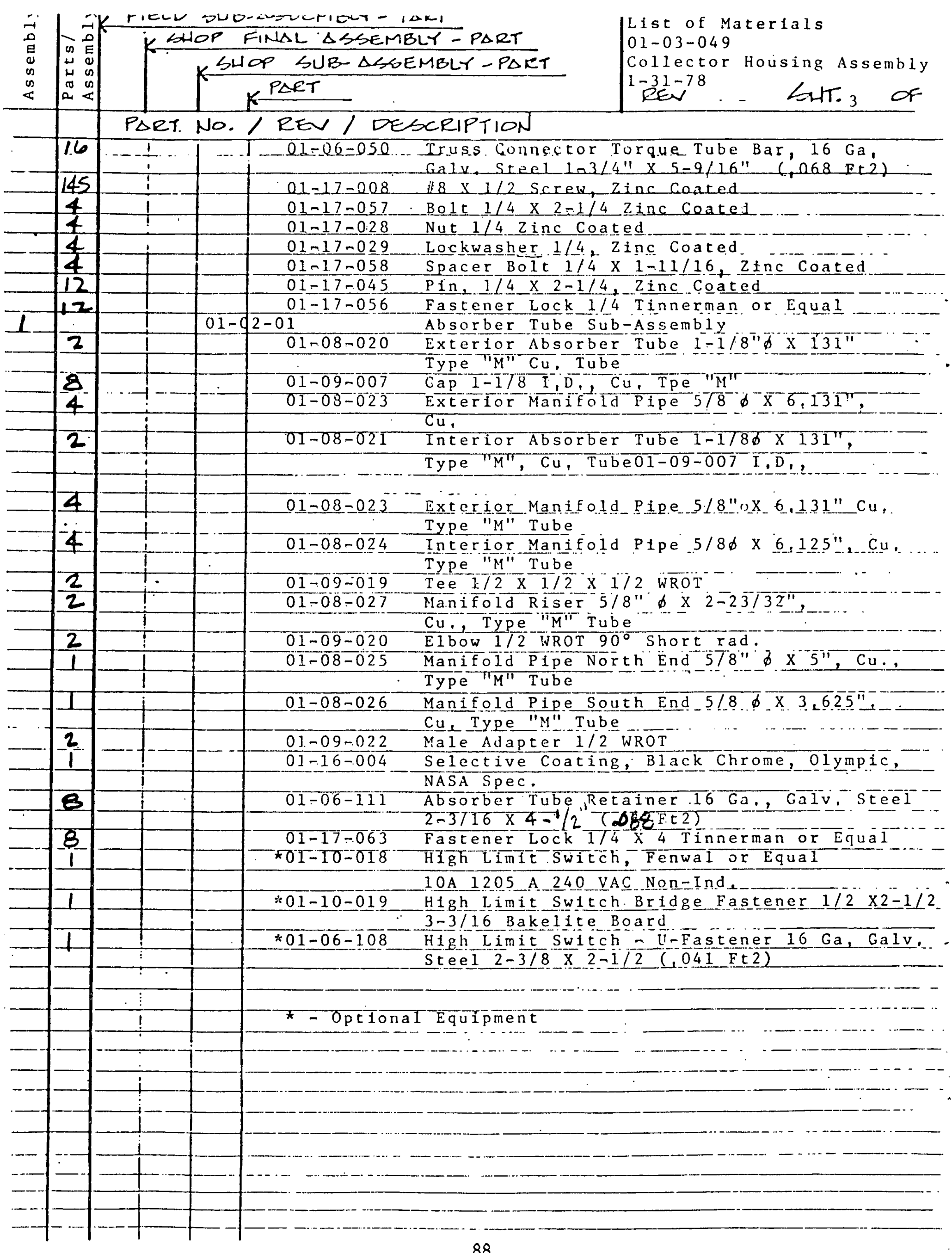



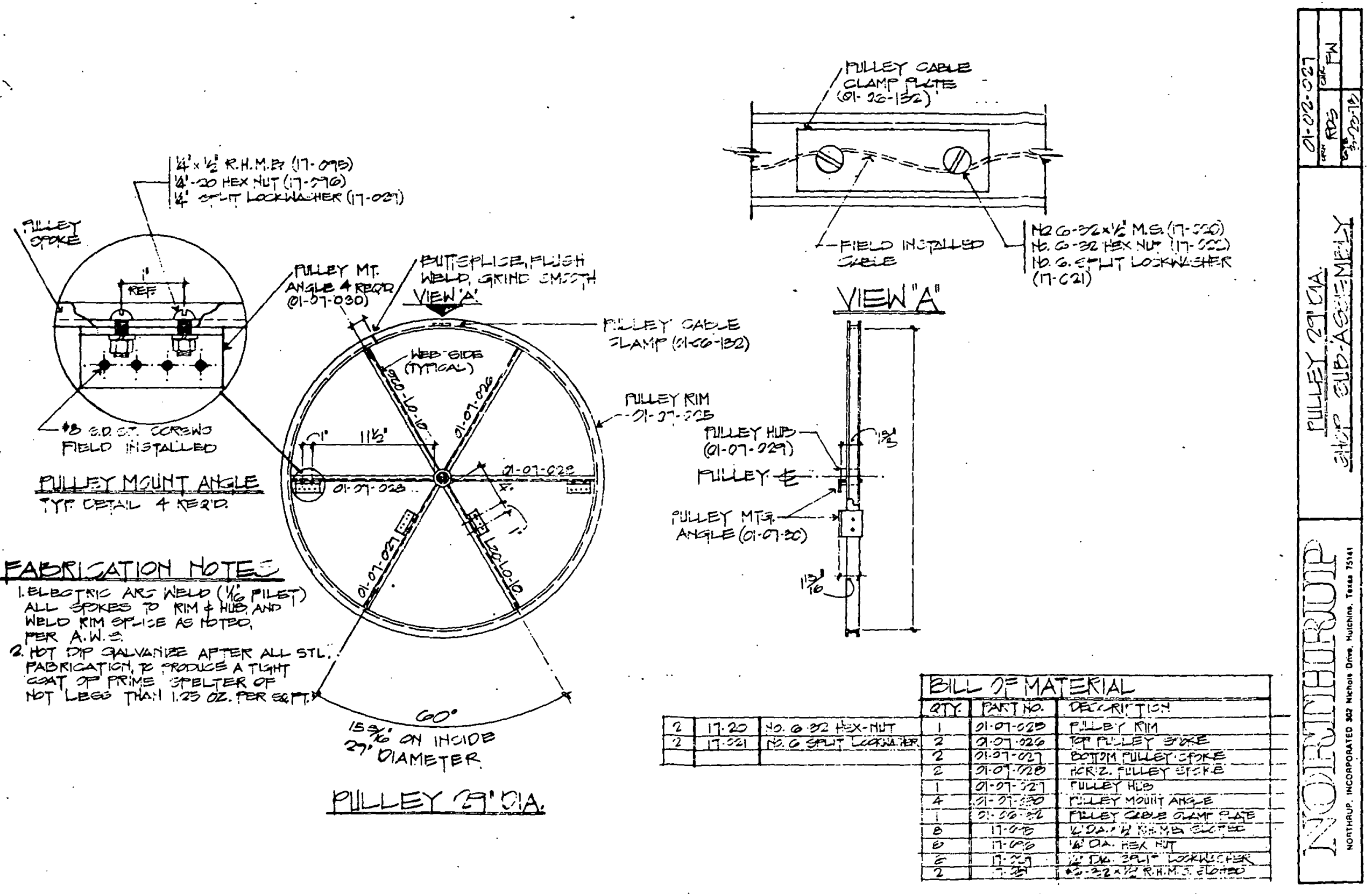

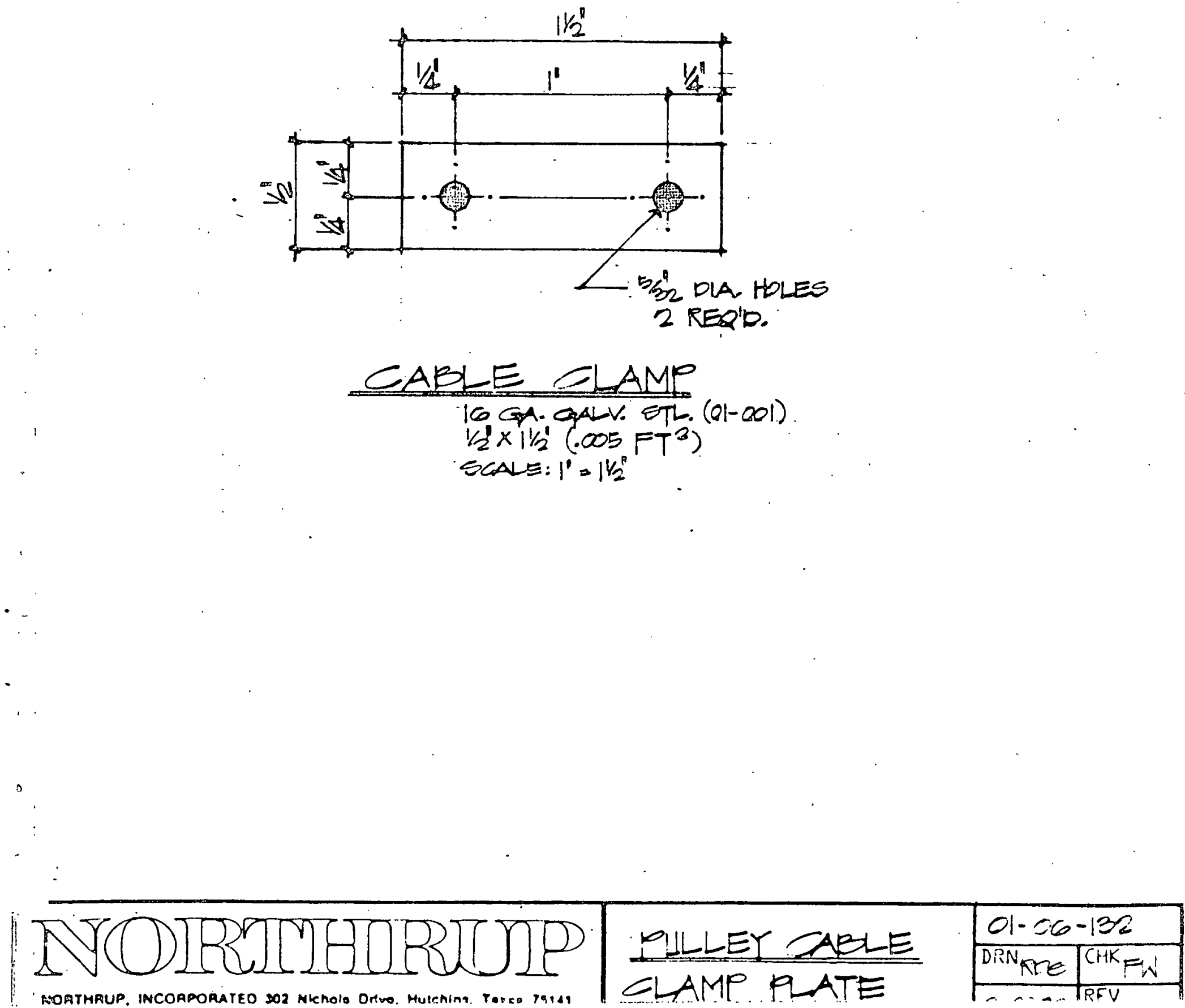


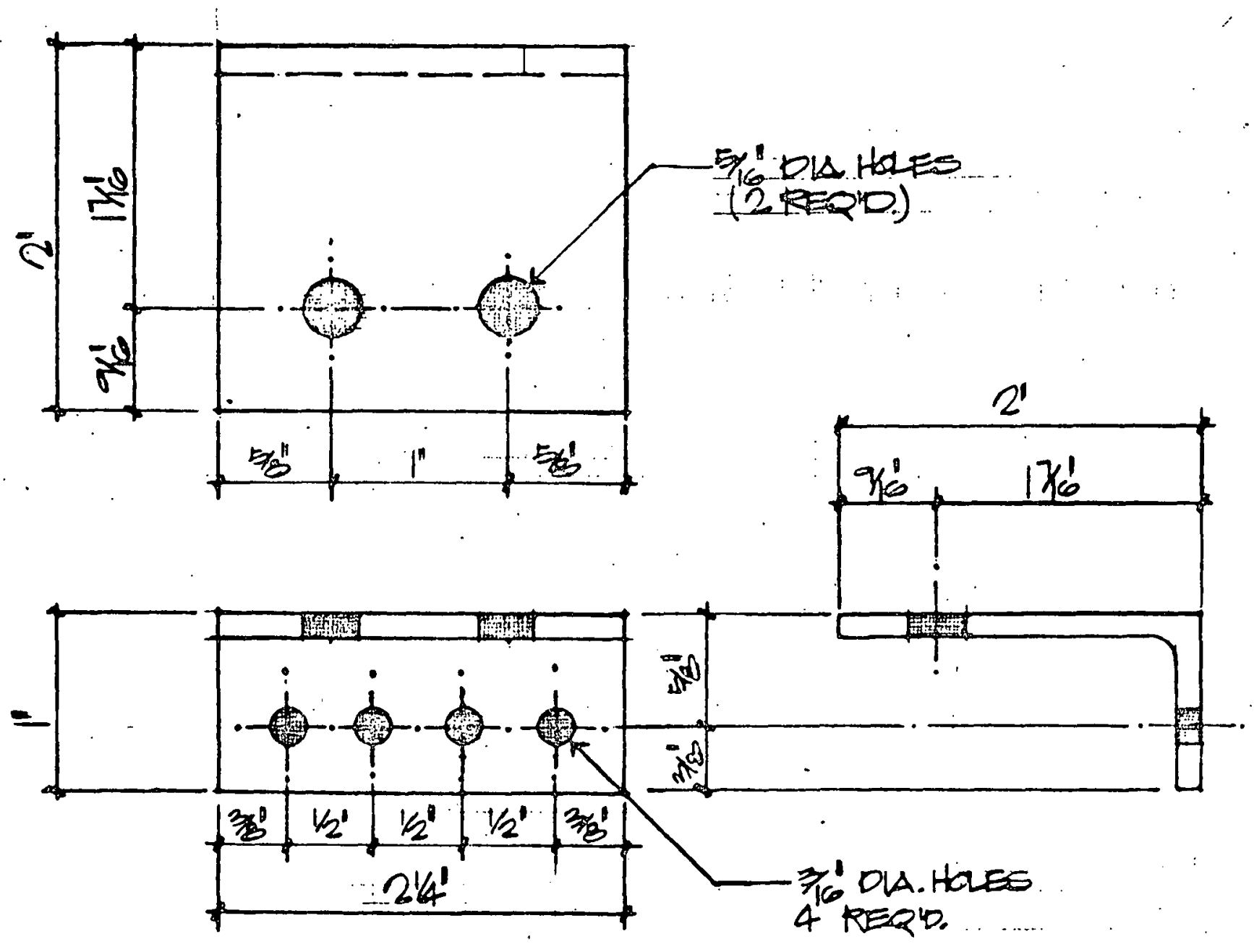

\section{PULLEY MONNT AMGLE

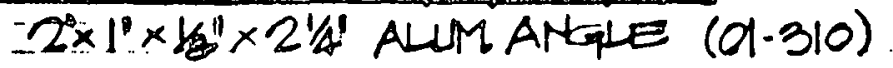 \\ . CO633-TS 0.491 LBS/FT. SQ CORNERS}




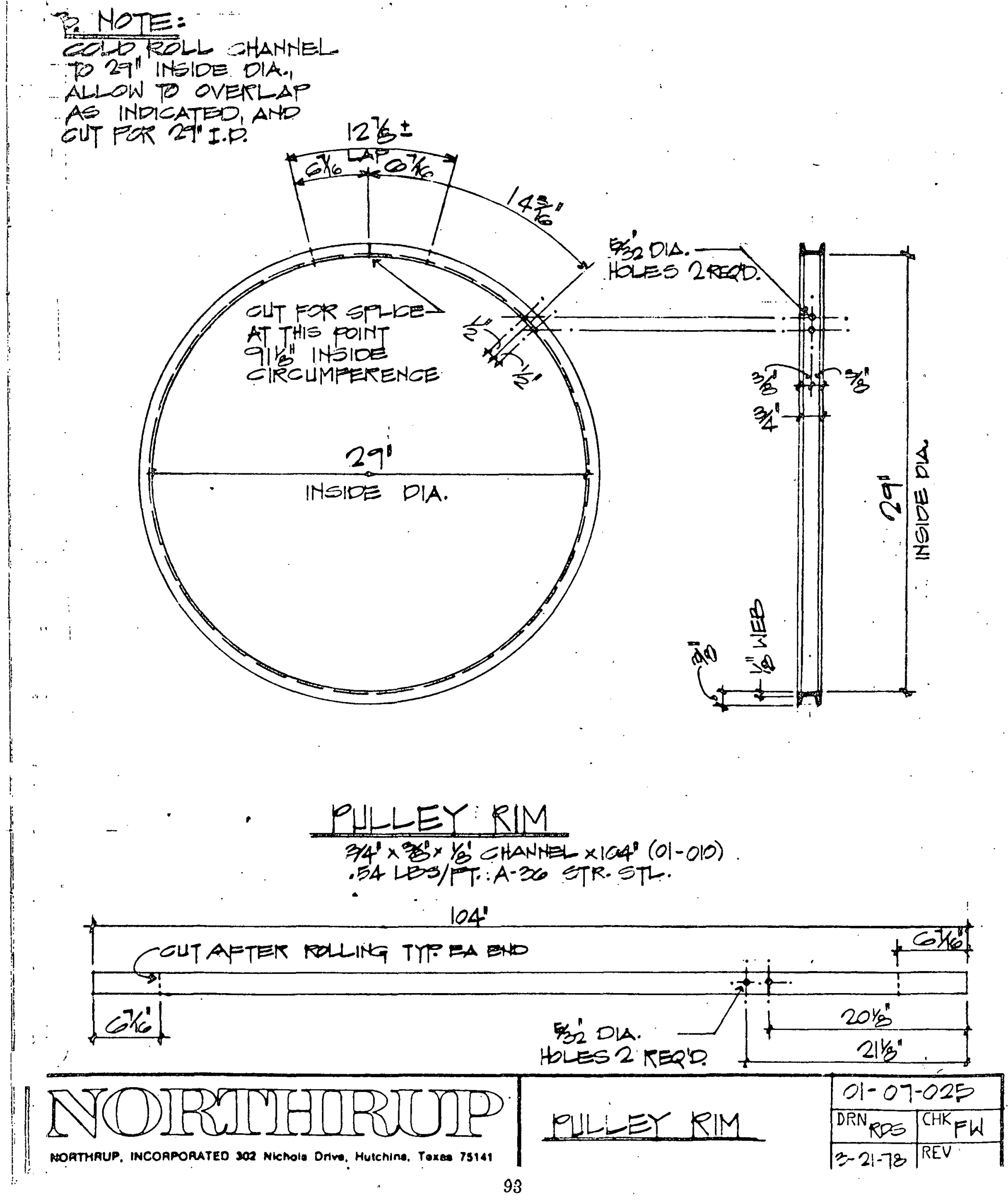



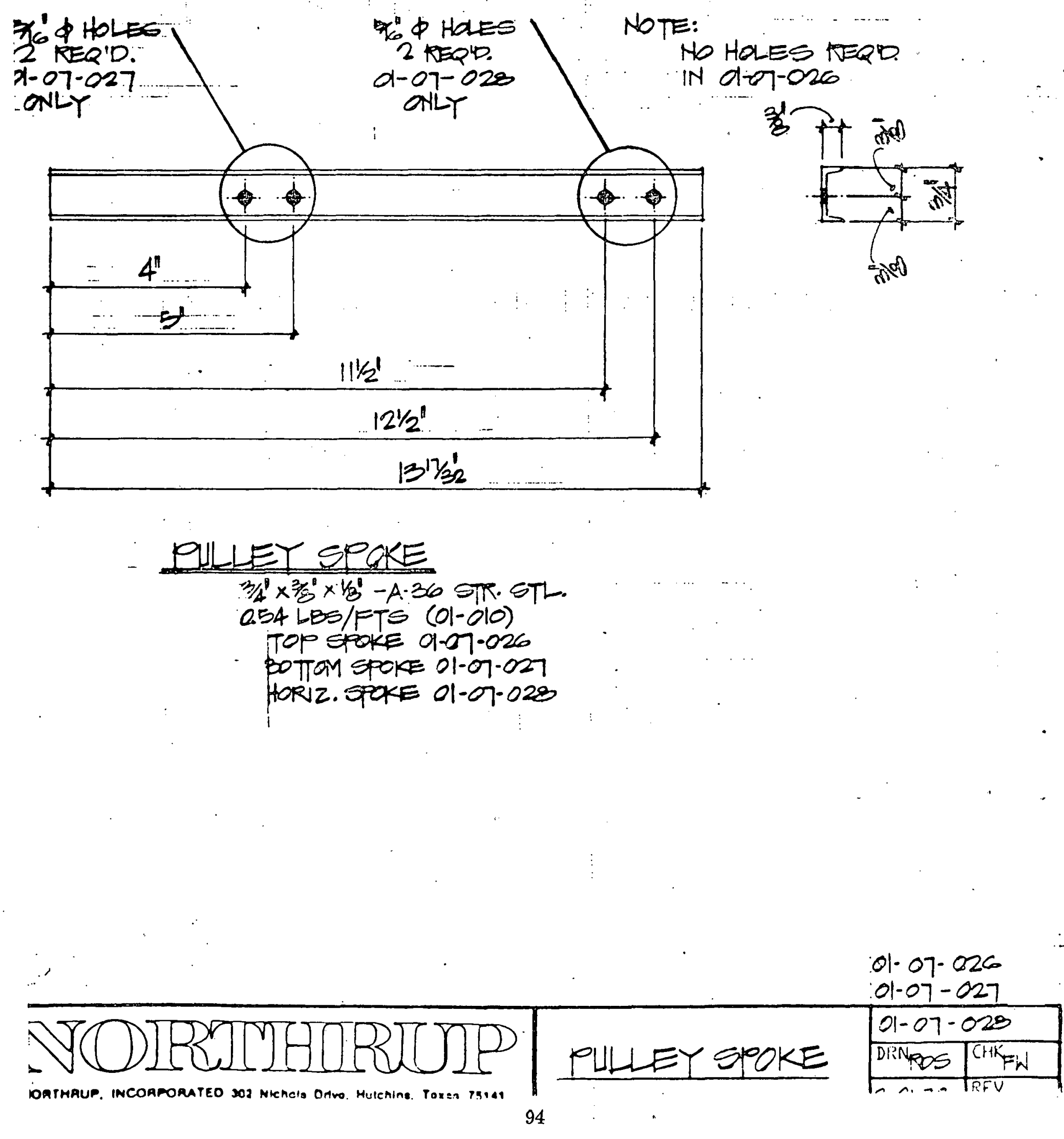


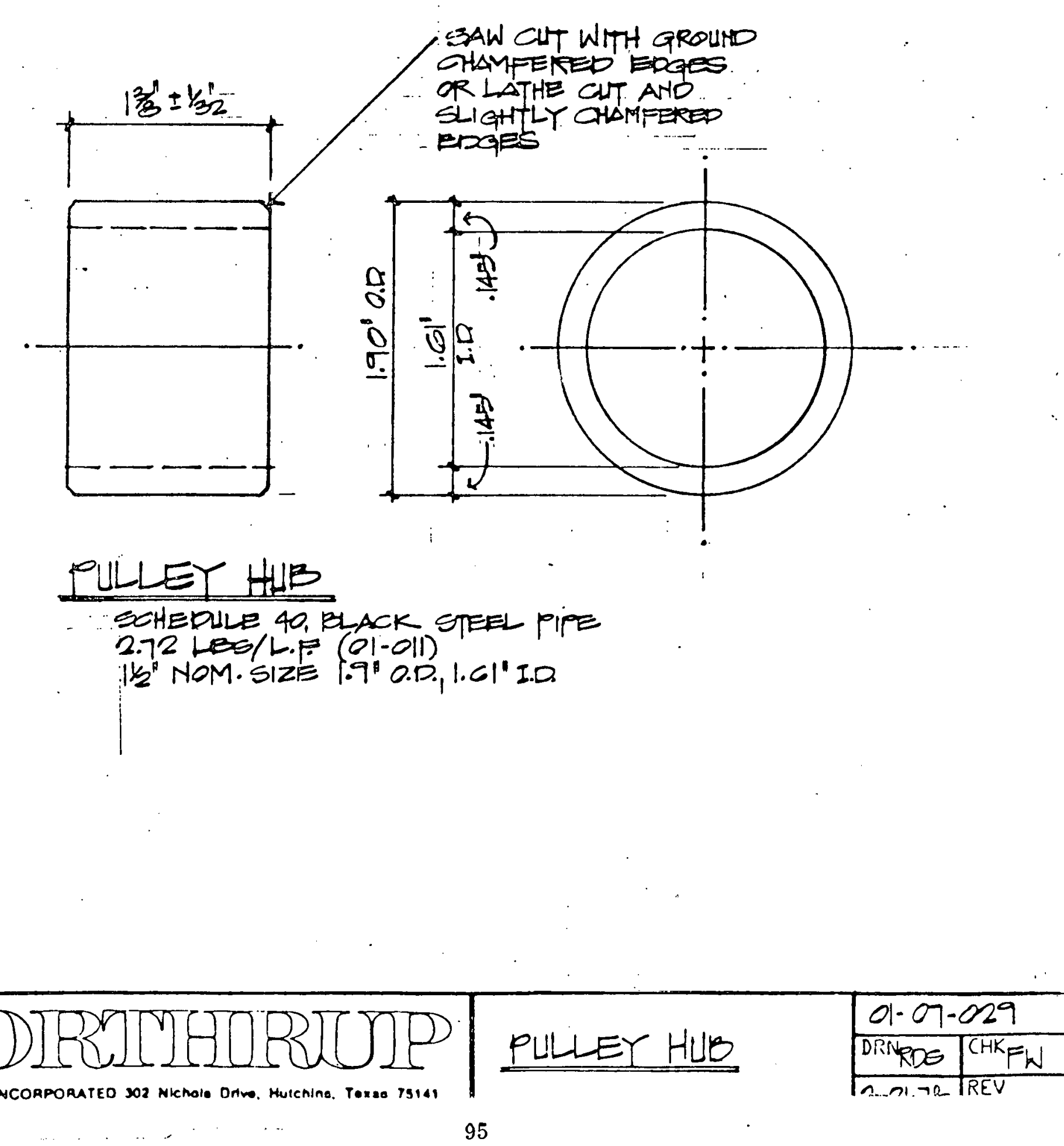




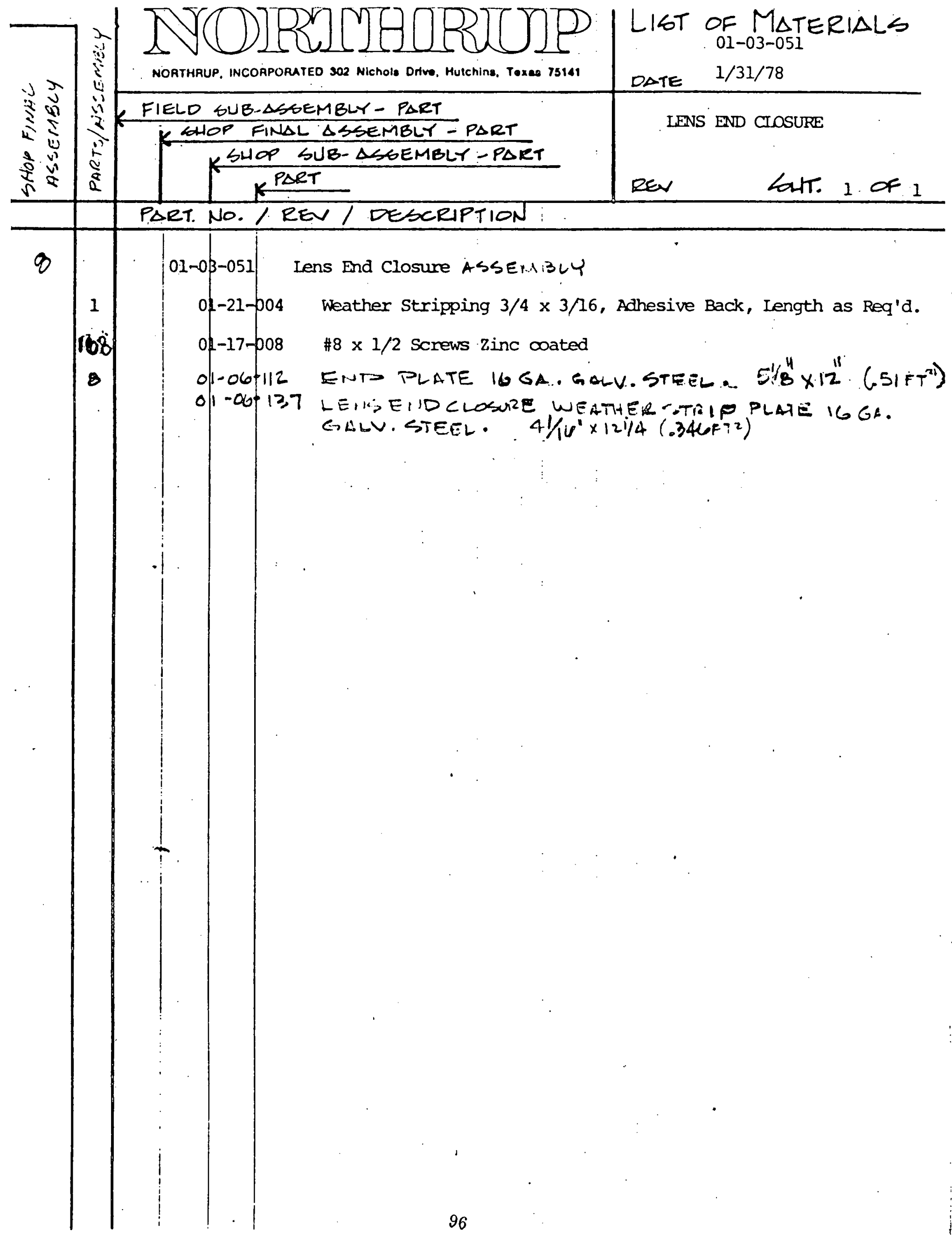




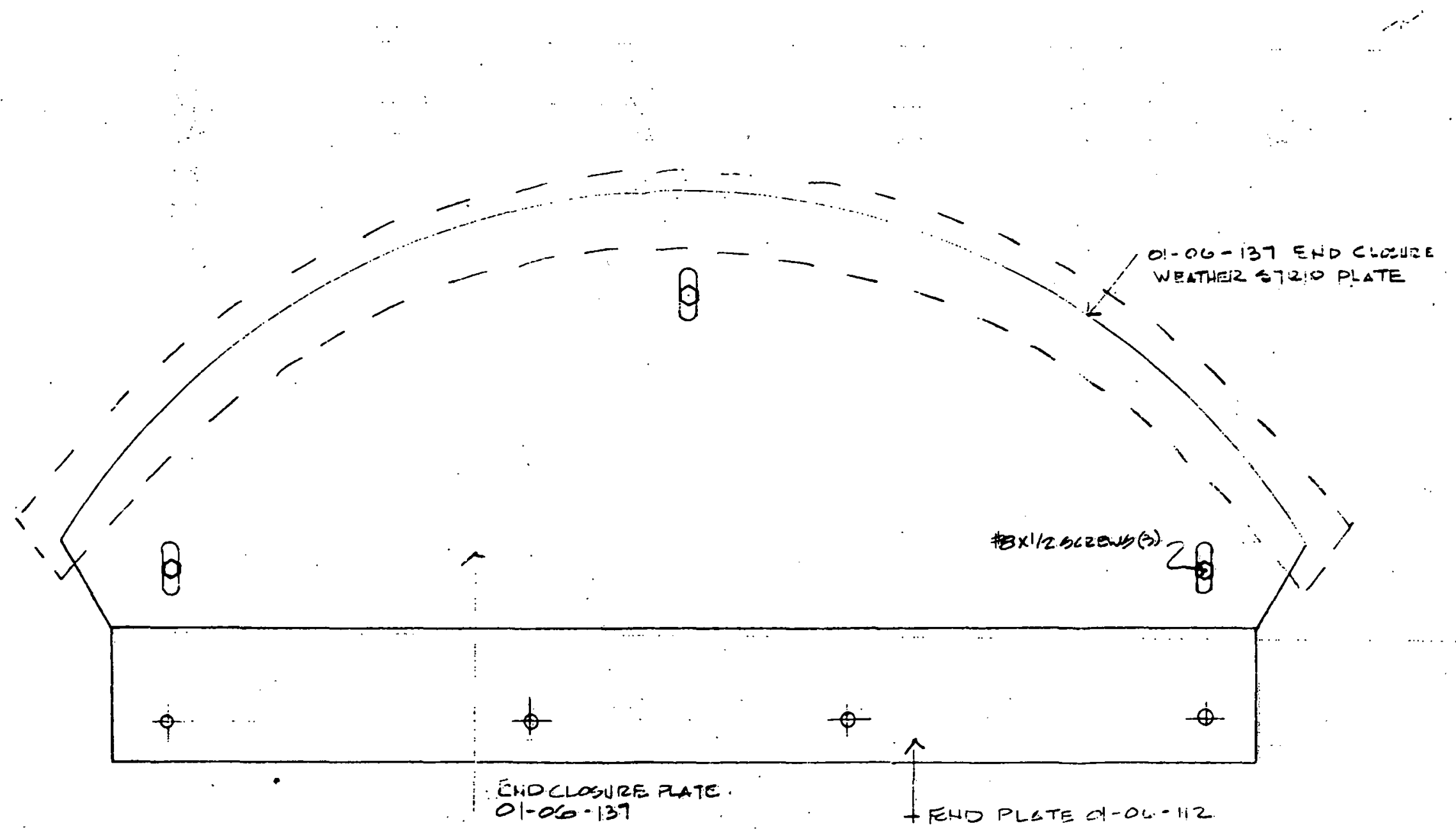

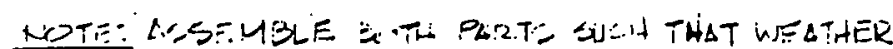

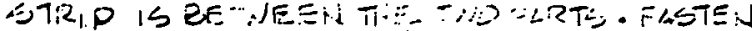

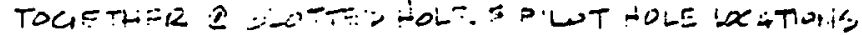

Tocir

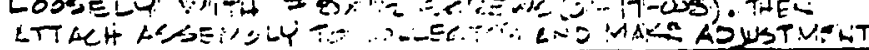

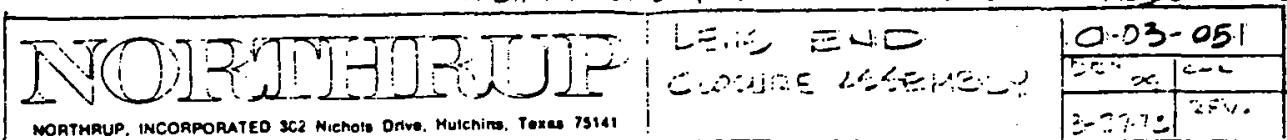




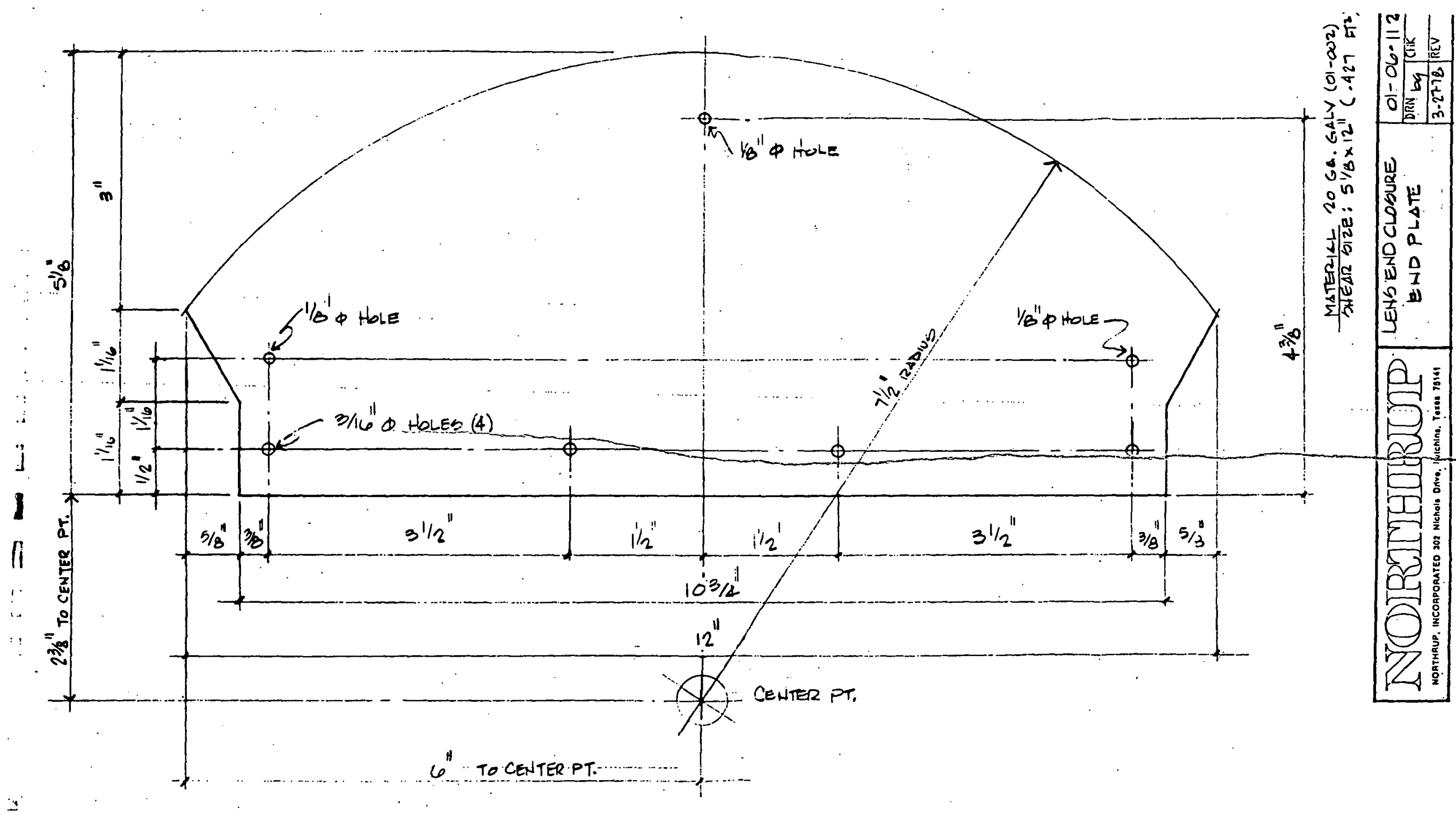




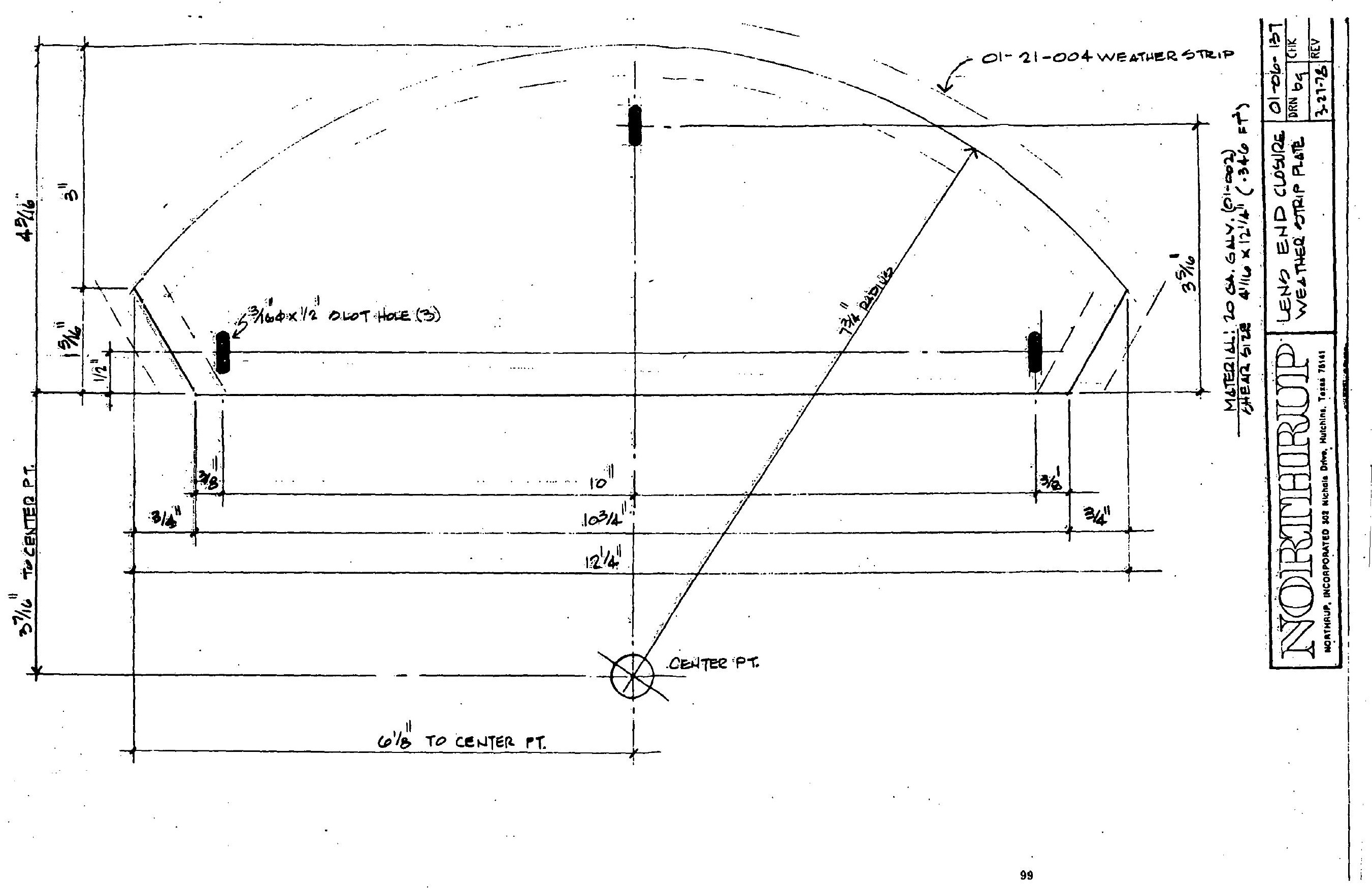




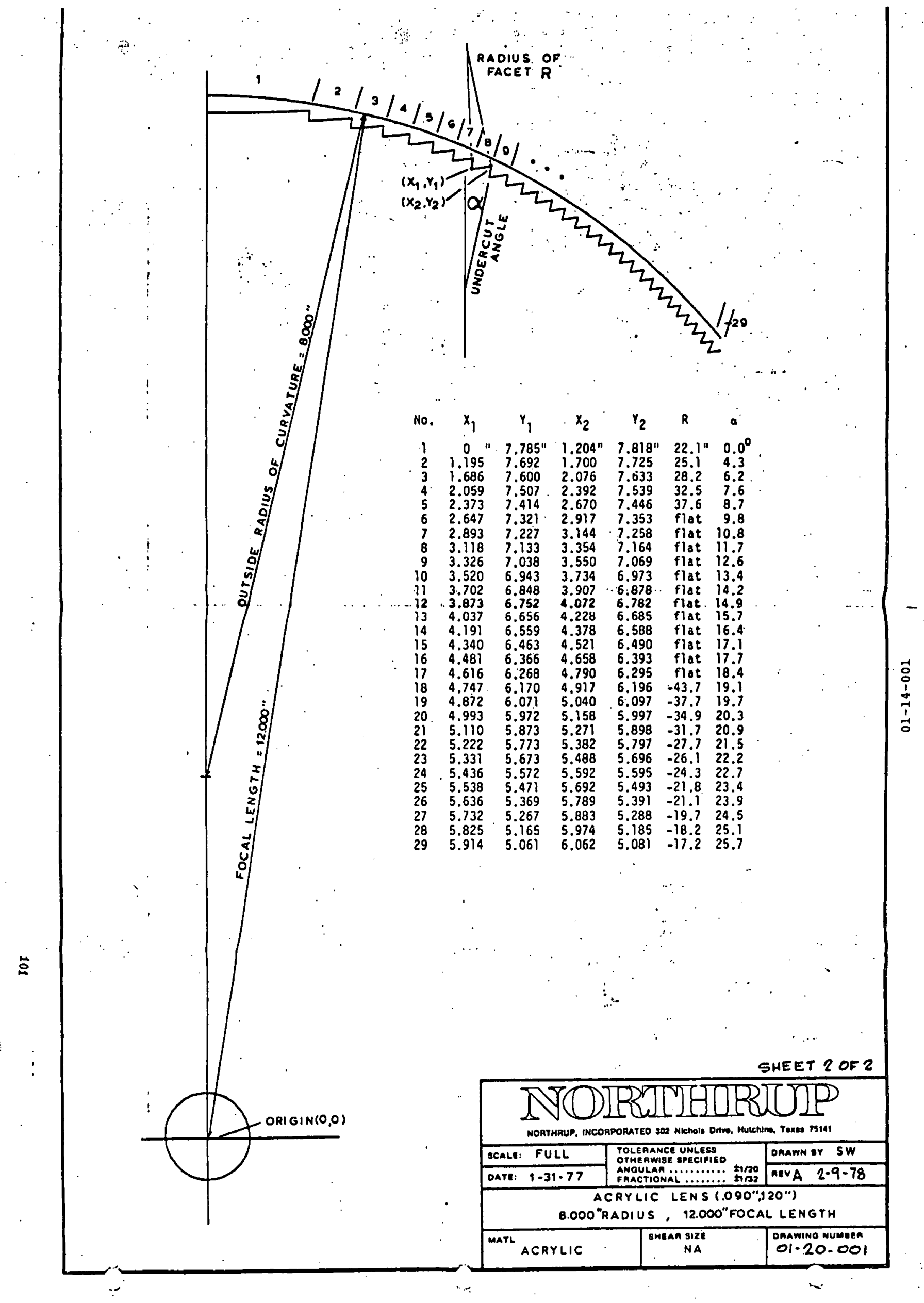



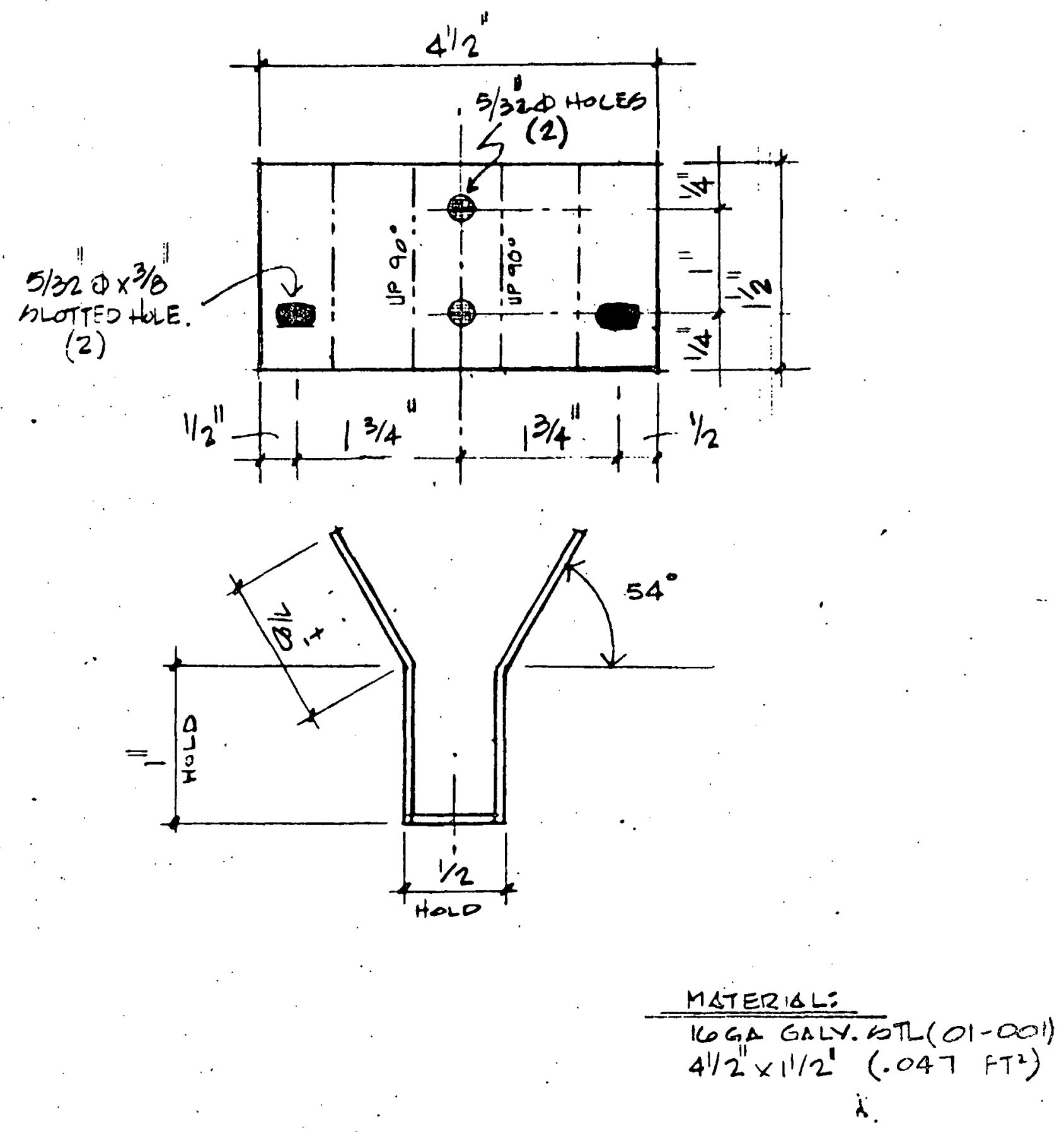

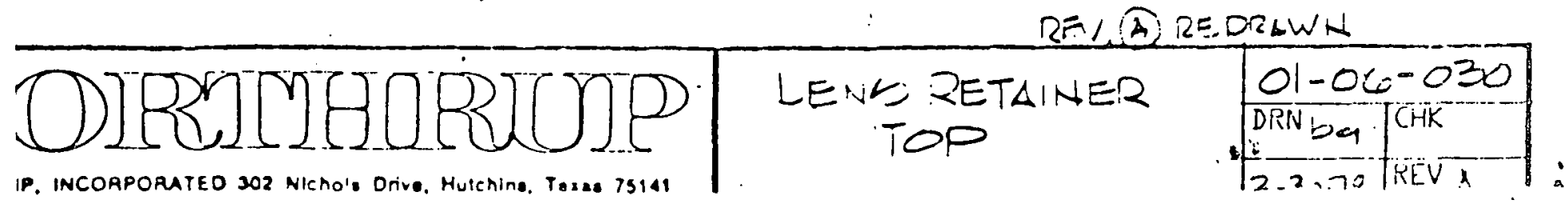

102 


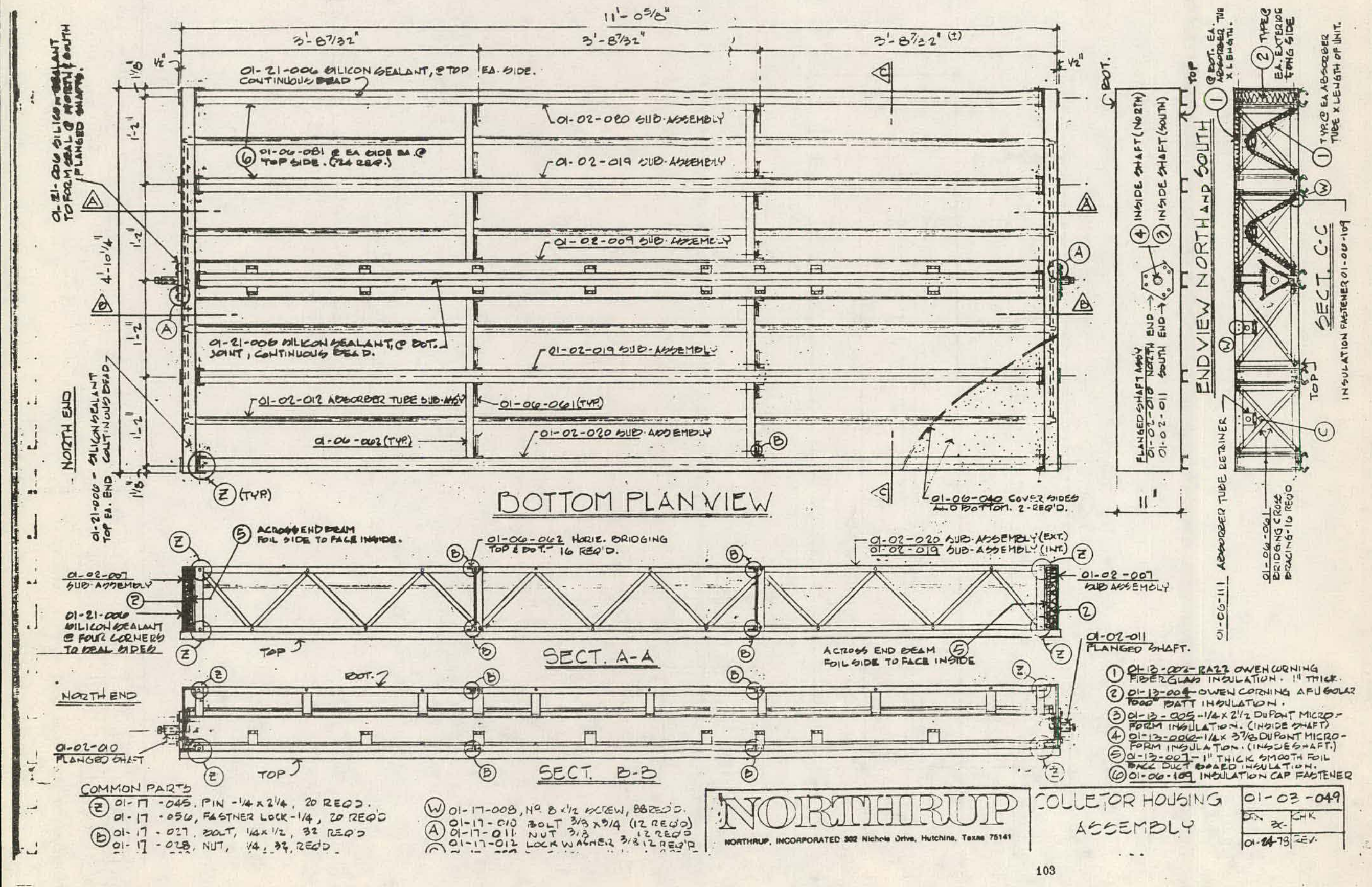



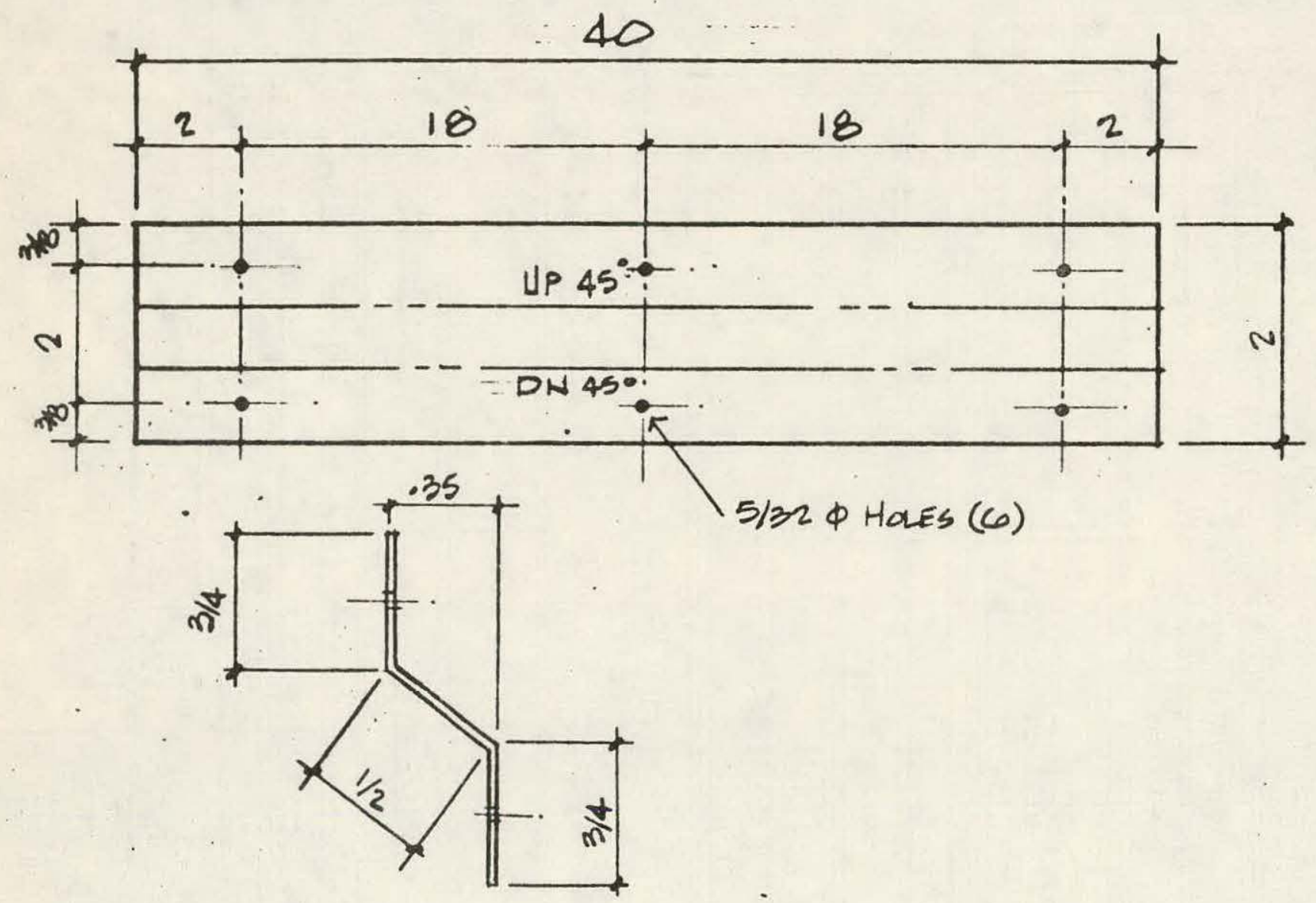

MATERIAL

20GA. GALVSTL. O1-01-DOl $2 \times 40 \quad\left(.55 \mathrm{ft}^{2}\right)$

$\ddot{\alpha}$.

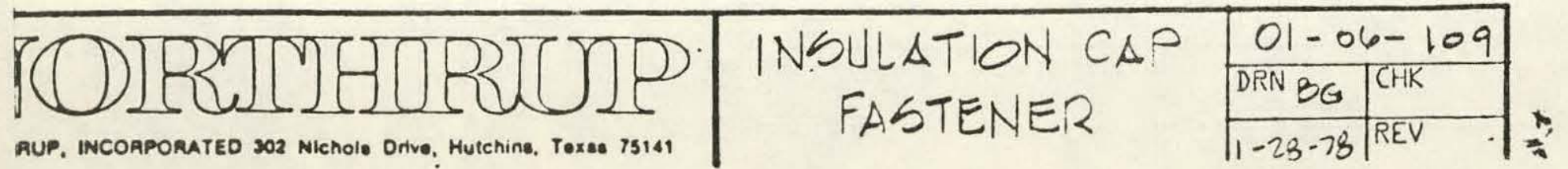




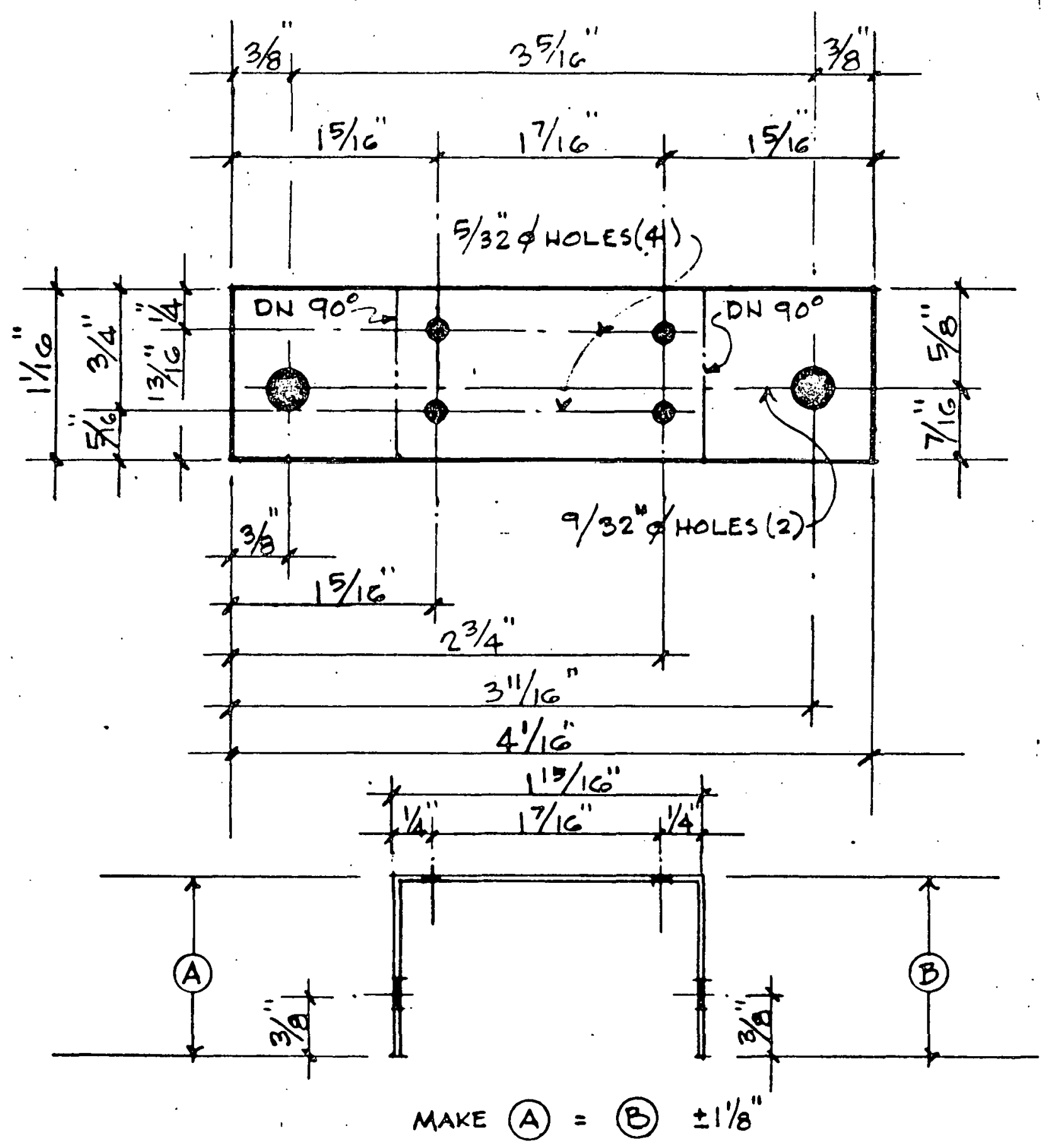

MATERIAL: 20 GALY, STL $(01.01 .002) 11 / 16^{\prime \prime} \times 41 / 16^{\prime \prime} \quad 0.030 \mathrm{FT}$

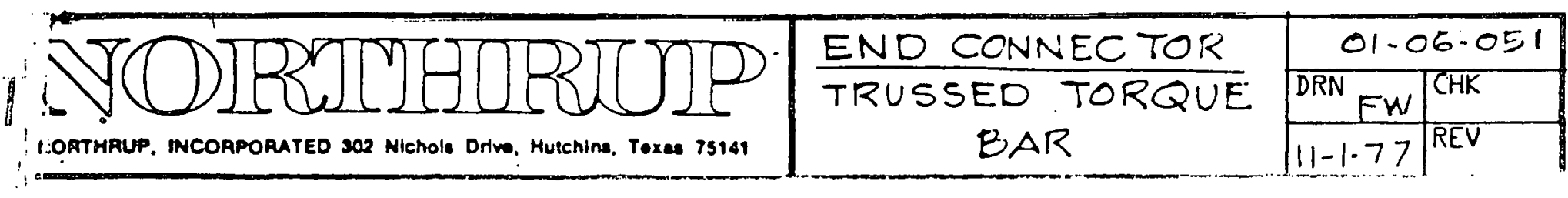

105 


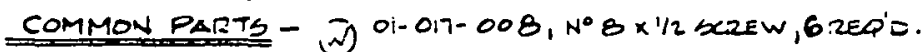

(X) $01-017-057$, BOLT $1 / 4 \times 21 / 4,2$ RED 0

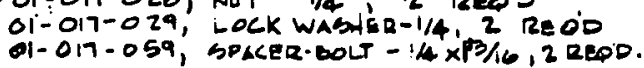

(Y) Ol-017-057, BOLT - 1/4 $\times 21 / 4,2$ REQ'O.

C1-017-026, NUT - $1 / 4,4$, 2 2 REQ'D.

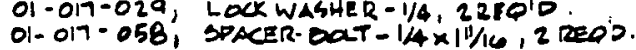

(2) $01-01-01-045, \quad A H-1 / 4 \times 21 / 4,12 R=Q^{\prime} D$.

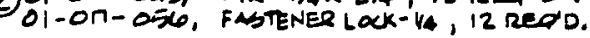

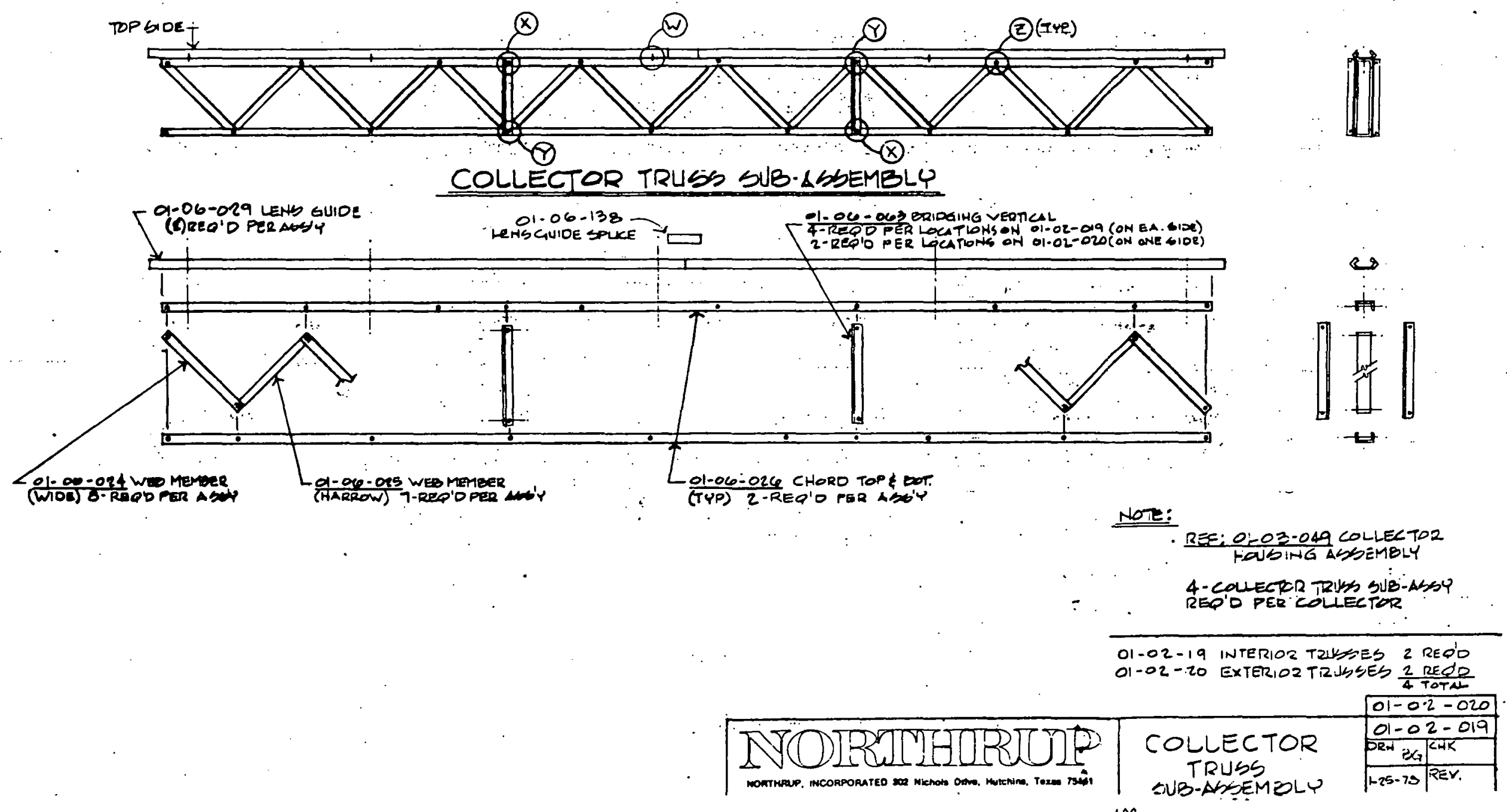



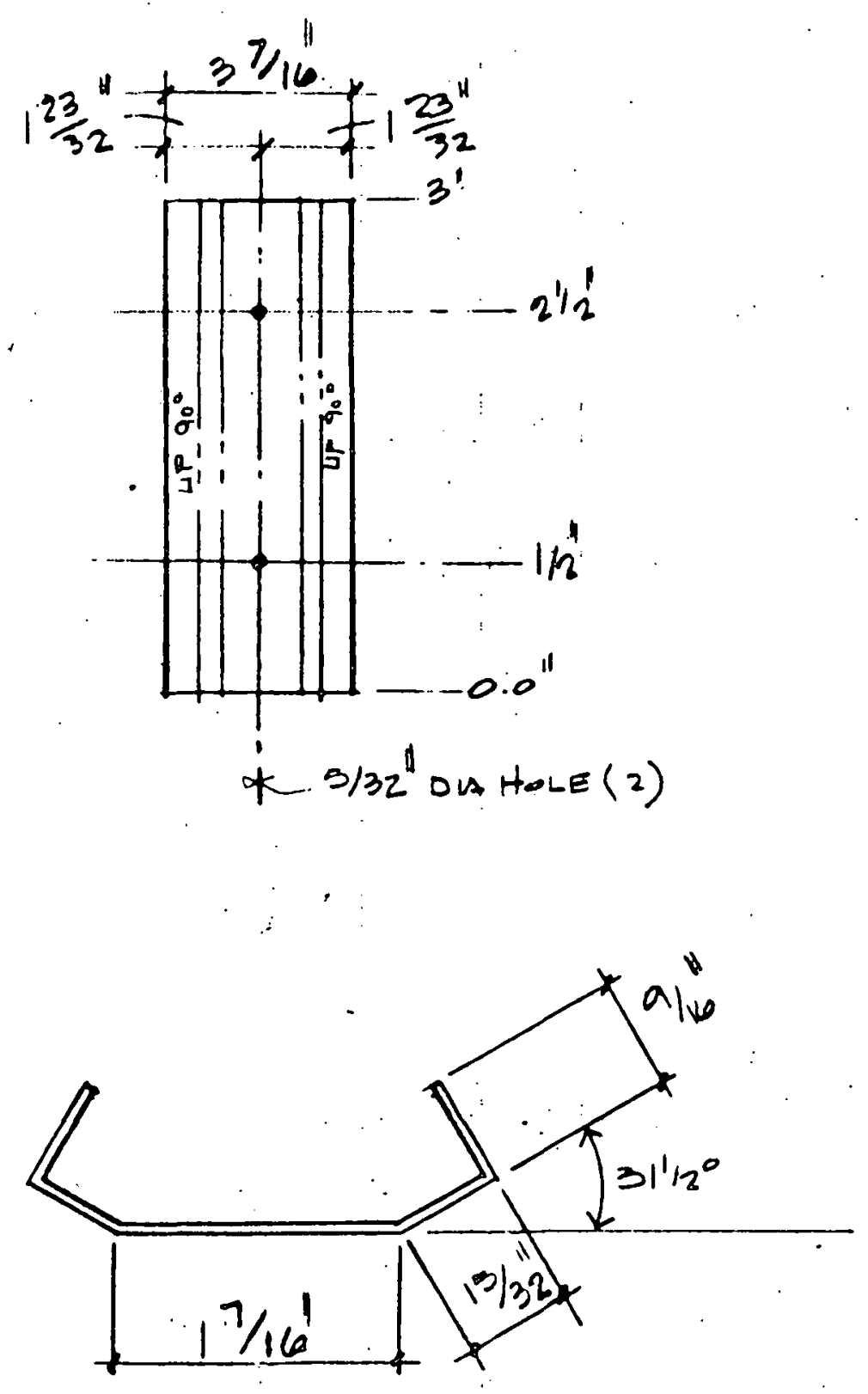

$$
\begin{aligned}
& M \triangle T E R, \triangle L=20 G 4 \text { GALV. BTL } \\
& 3716^{11} \times 3^{11}\left(.072 \mathrm{FT}^{2}\right)
\end{aligned}
$$

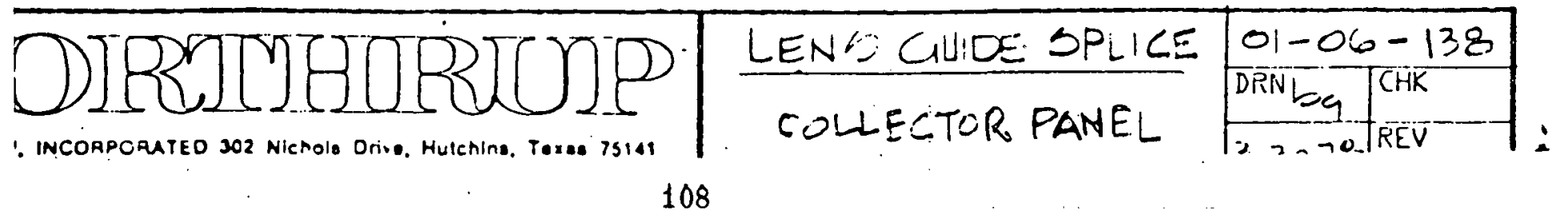



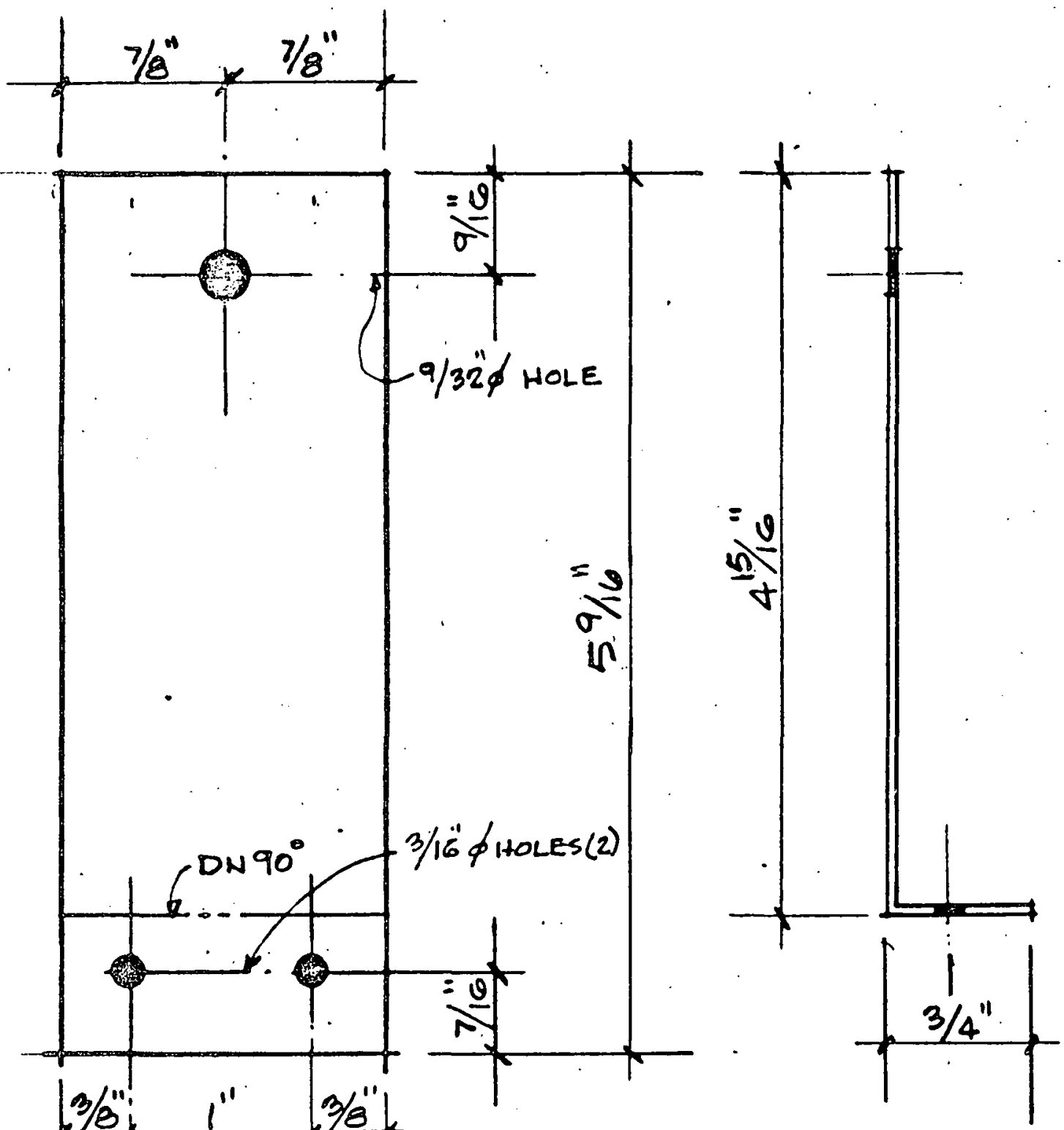

MATERIAL: 16 GA GNV. STL (01.01-001) $13 / 4^{\prime \prime} \times 5 \% 160,068 \mathrm{FT}^{2}$

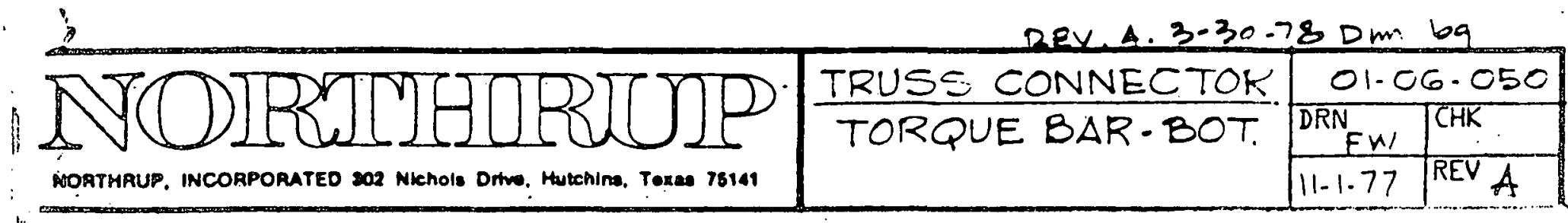

109 

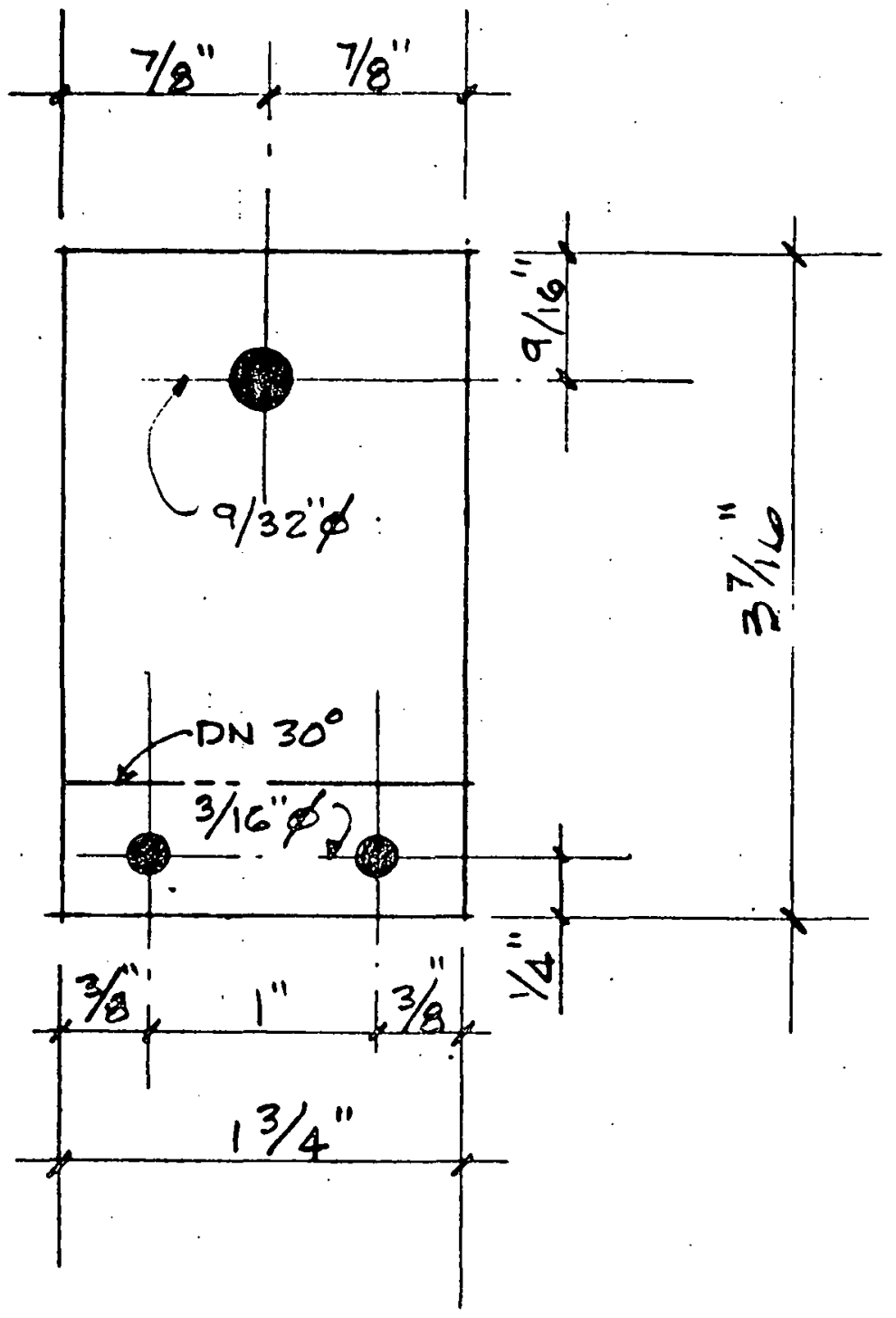

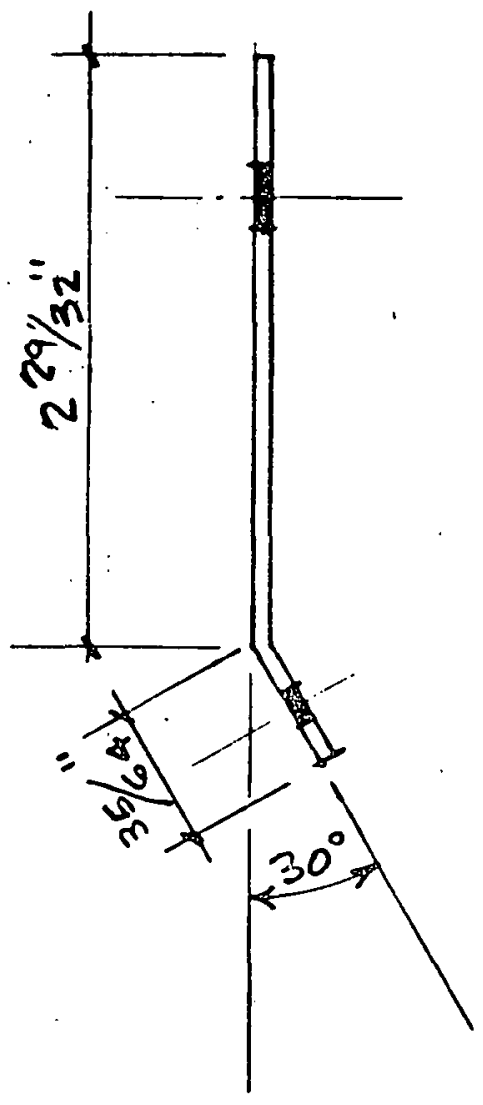

MATERIAL: IGGA.GALV. STL. (01.01.001) 13/4"×3\%"B $0.042 \mathrm{FT}^{2}$

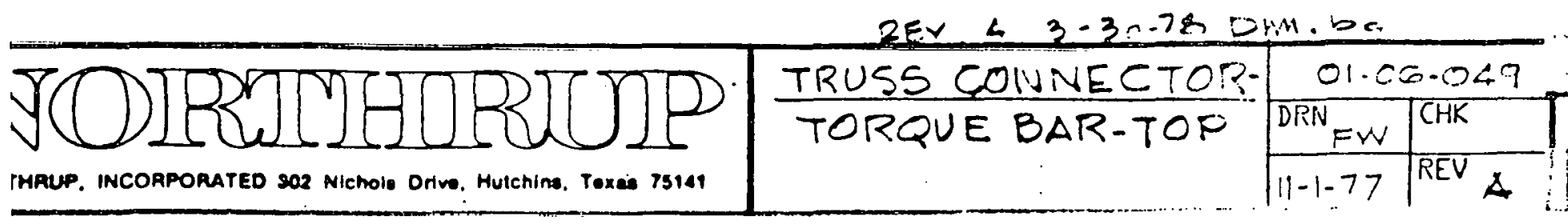




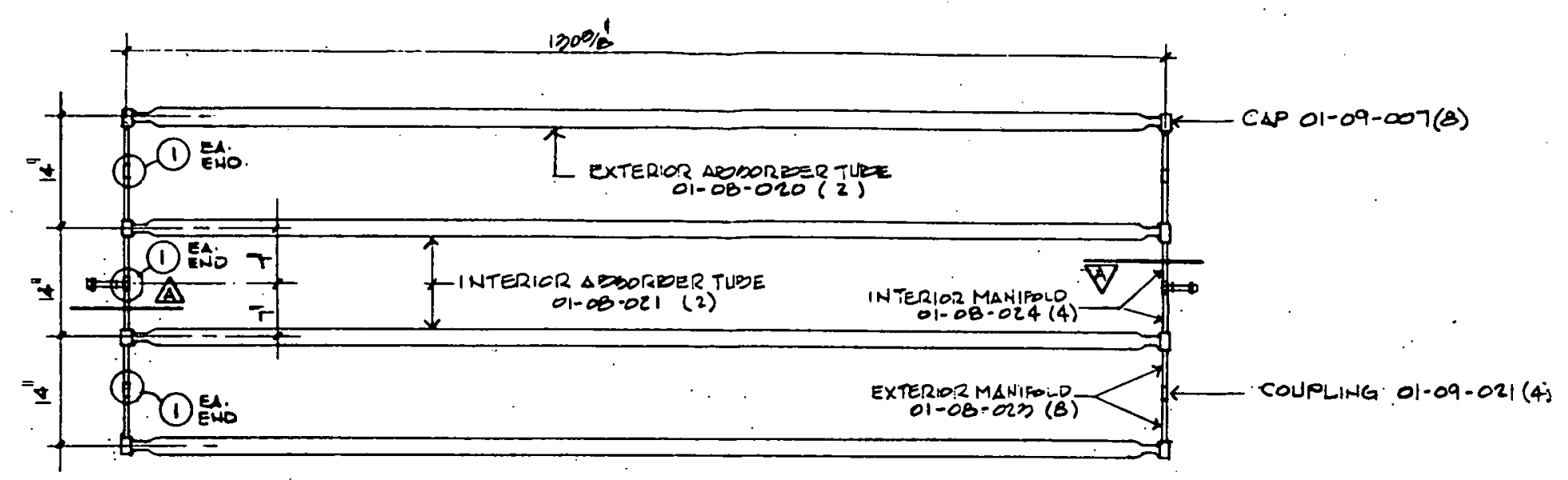

ABSORBER TUBE SHB ASSEMBLY

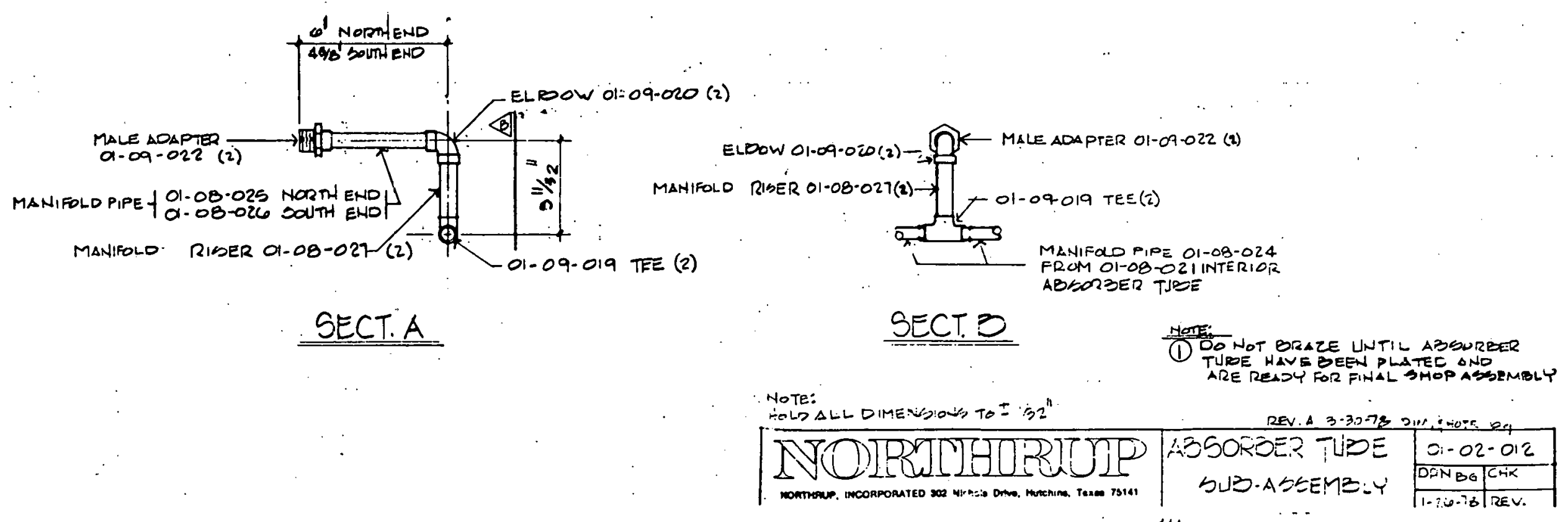




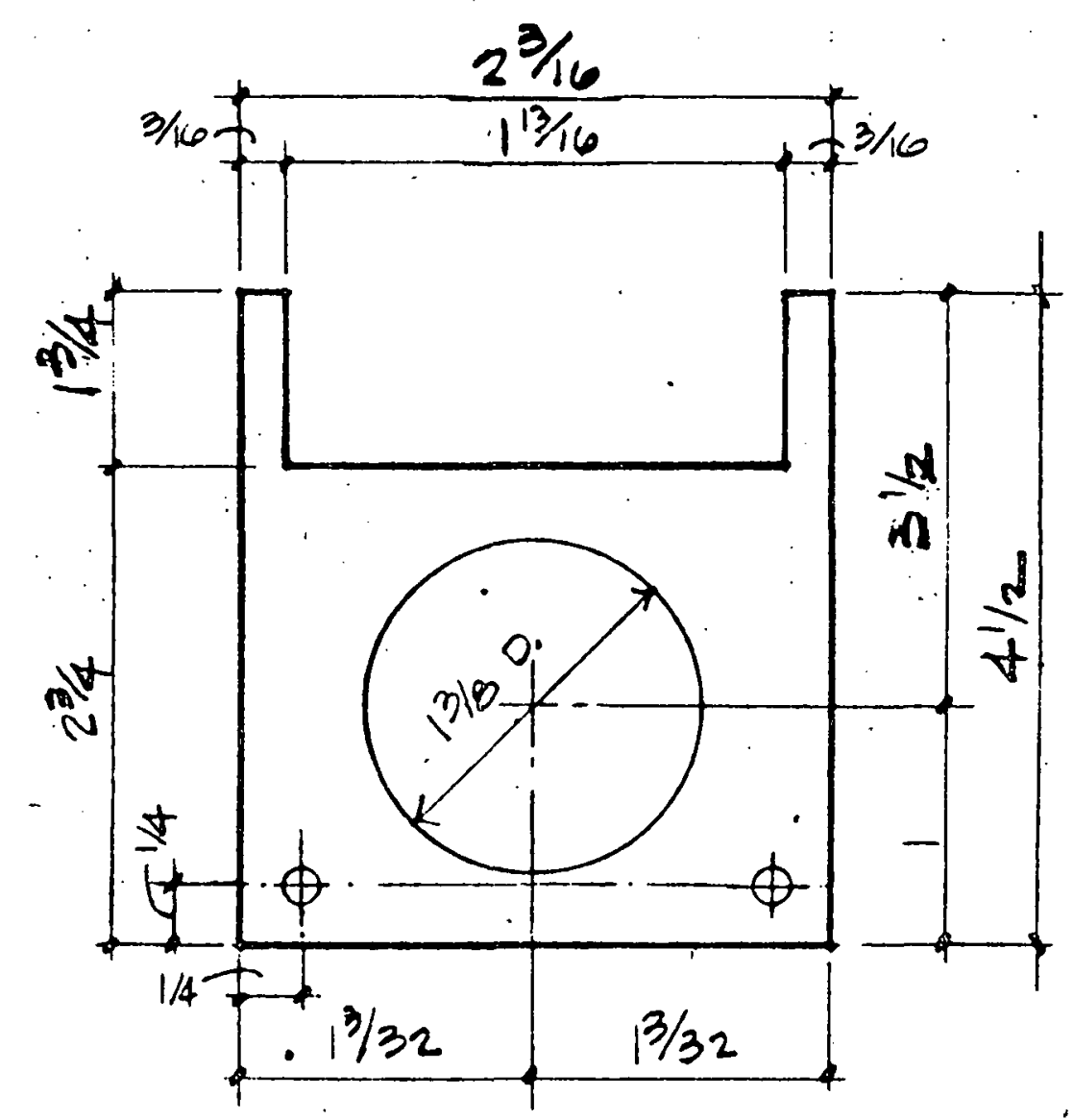

$$
\begin{aligned}
& \text { MATERIAL: } \\
& 16 G G \Delta \cdot G \Delta L V \cdot G T L \cdot(01-01-001) \\
& 23 / 16 \times 4 V_{2}^{18} \cdot\left(.063 .97^{2}\right)
\end{aligned}
$$

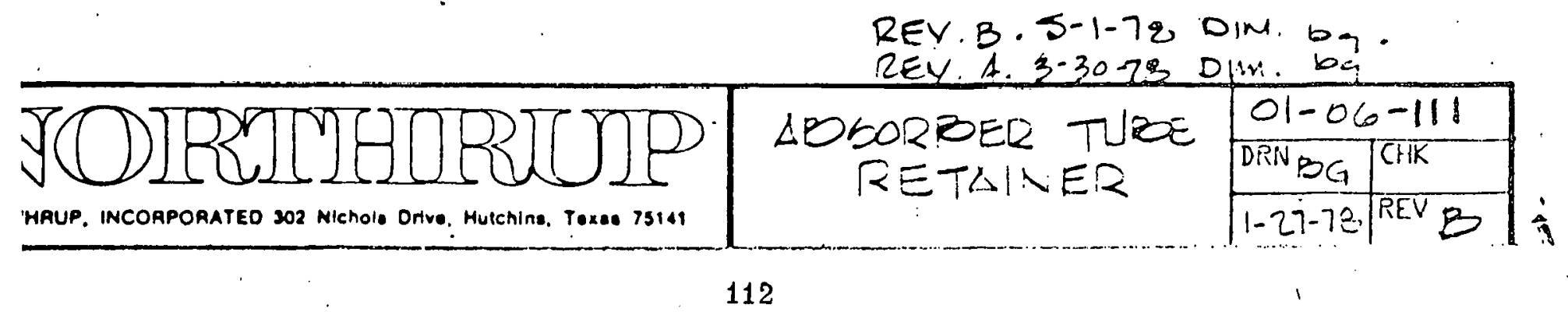




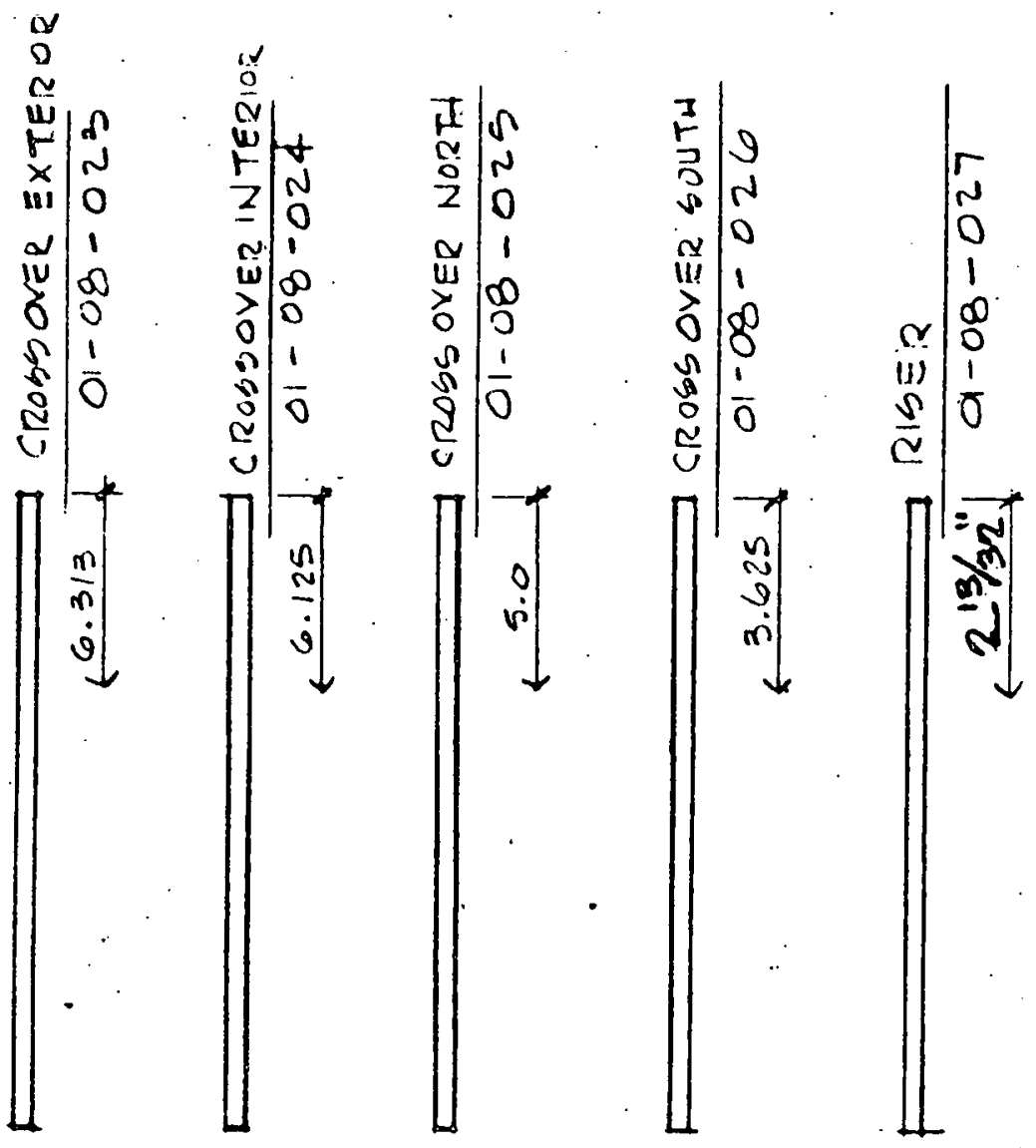

\begin{tabular}{|c|c|c|}
\hline TYFE & & QTY \\
\hline CROSSOV & EXT.- & 8 \\
\hline$: 11$ & INT. - & 4 \\
\hline 11 & NORTL & 1 \\
\hline 11 & South- & ! \\
\hline RISER ... & 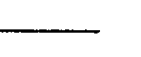 & 2 \\
\hline
\end{tabular}

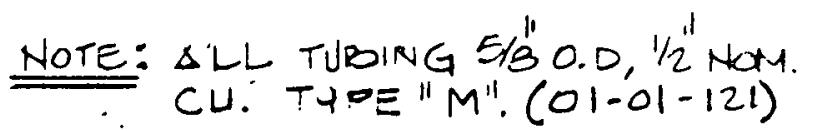

$\dot{h}$.

BeV. A 3-30-78 Dim. ba DV(D) 


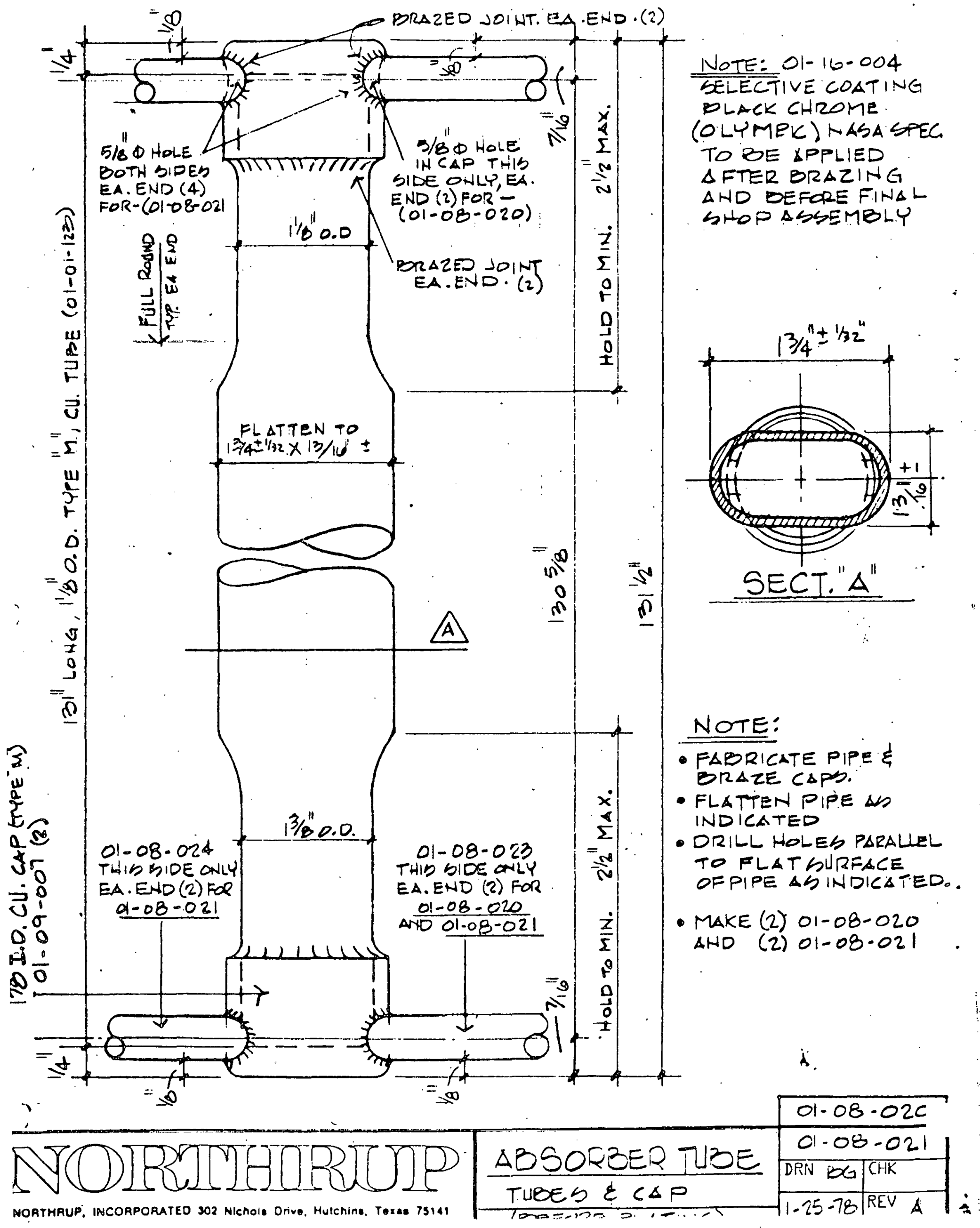




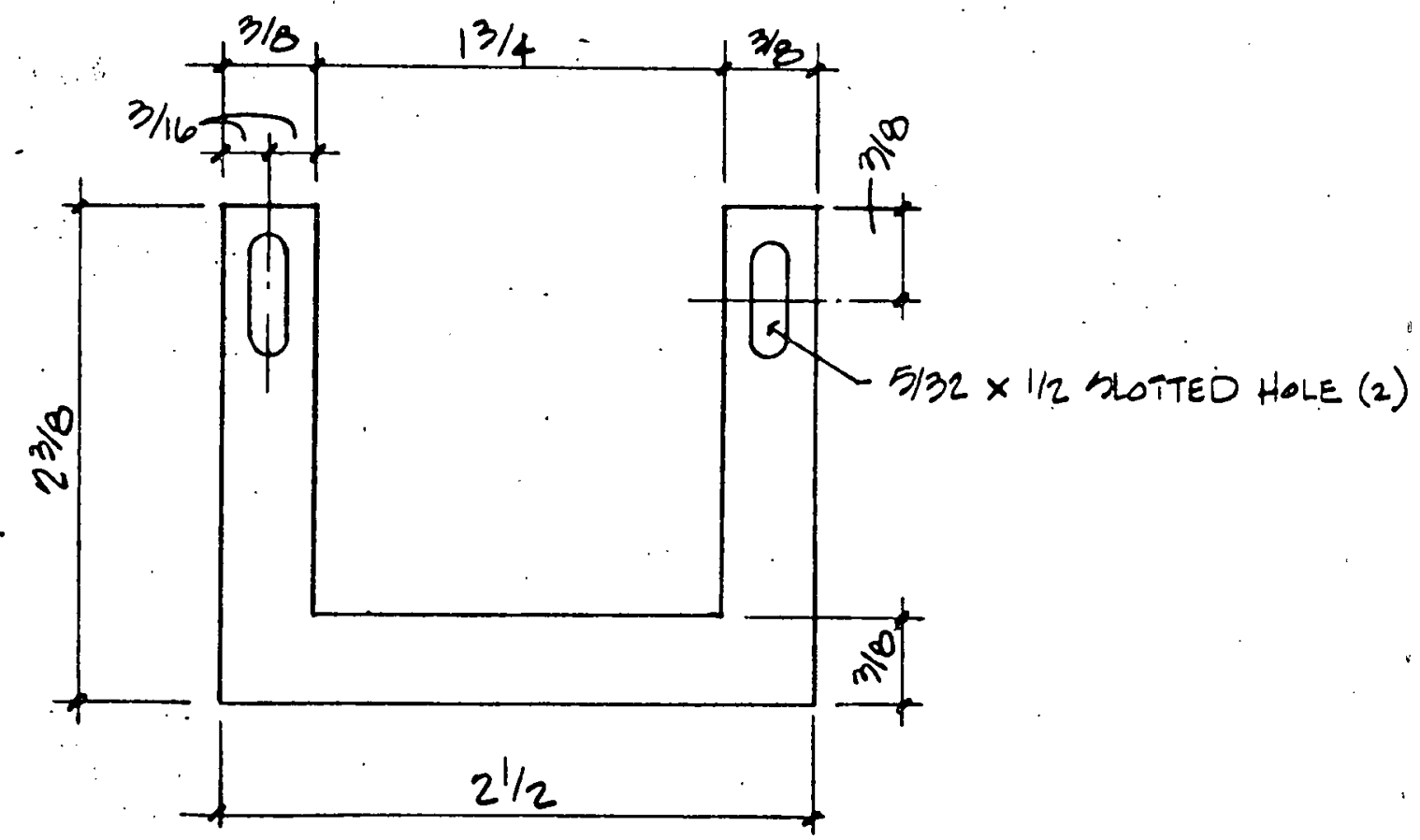

MATERIAL:

16 GA. GALV. (01-01-001)

. $23 / 8 \times 21 / 2\left(.041 \mathrm{ft}^{2}\right)$

I RECP'O

TOBE UBED WITH OI-IO-OIG

HICH LIMITSWITCH U-FASTENER

\begin{tabular}{|c|c|}
\hline \multicolumn{2}{|c|}{$01-06-108$} \\
\hline DRN $3 G$ & $\mathrm{CHK}$ \\
\hline $1-3 i-78$ & REV \\
\hline
\end{tabular}




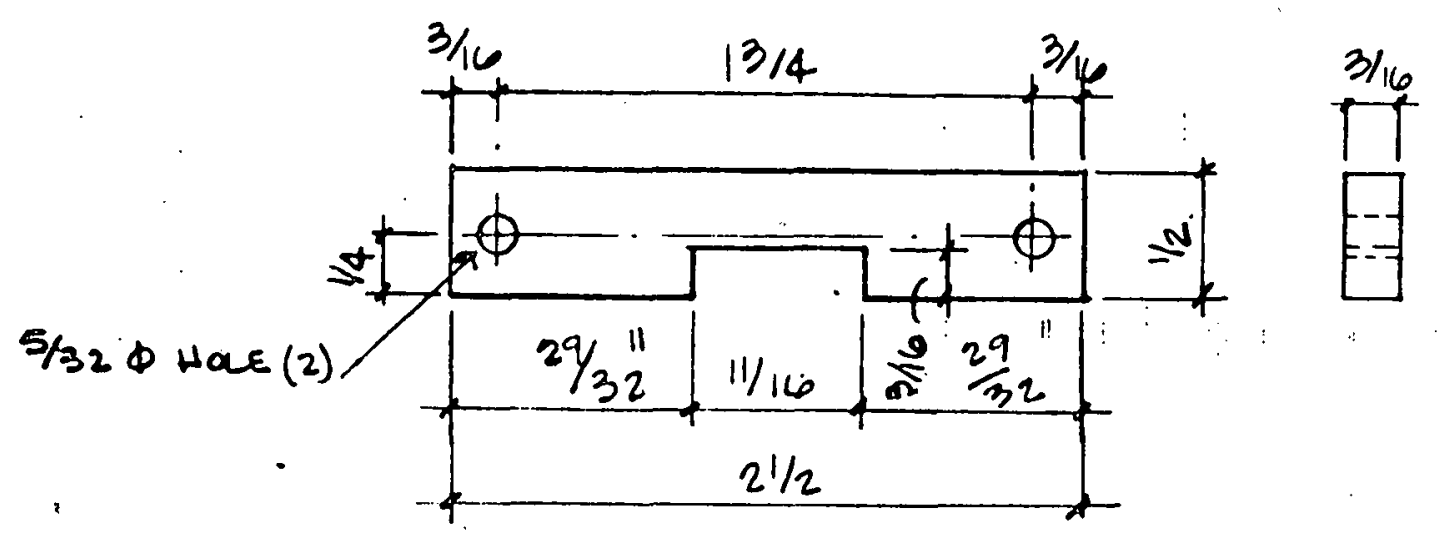

MATERIAL:

$1 / 2 \times 21 / 2 \times 1 / 4$ BAKELITE BOARD

1 REQ'O

TOBE UEE WITH O1-06-108

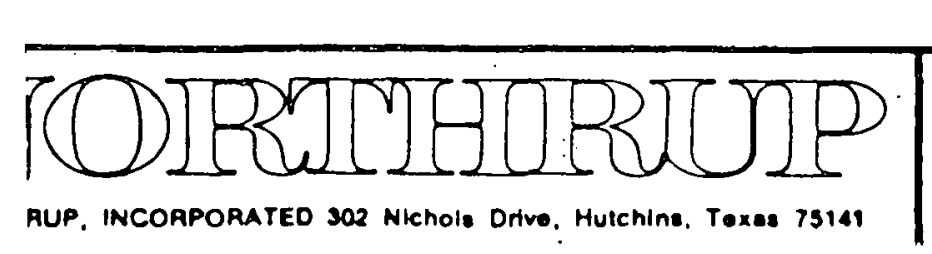

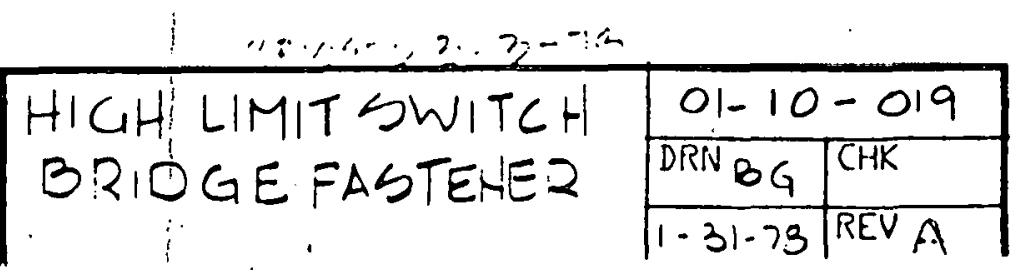

116 


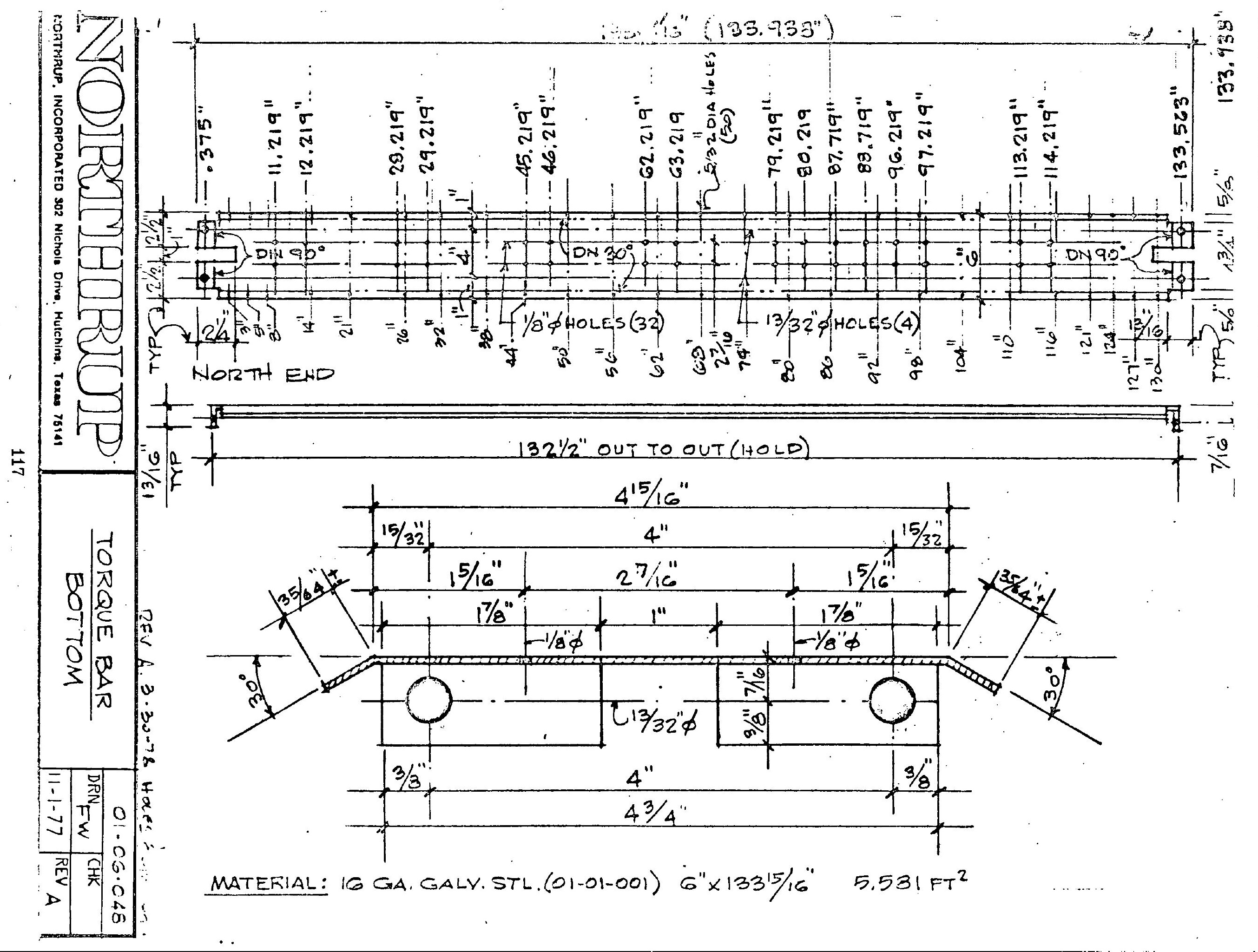




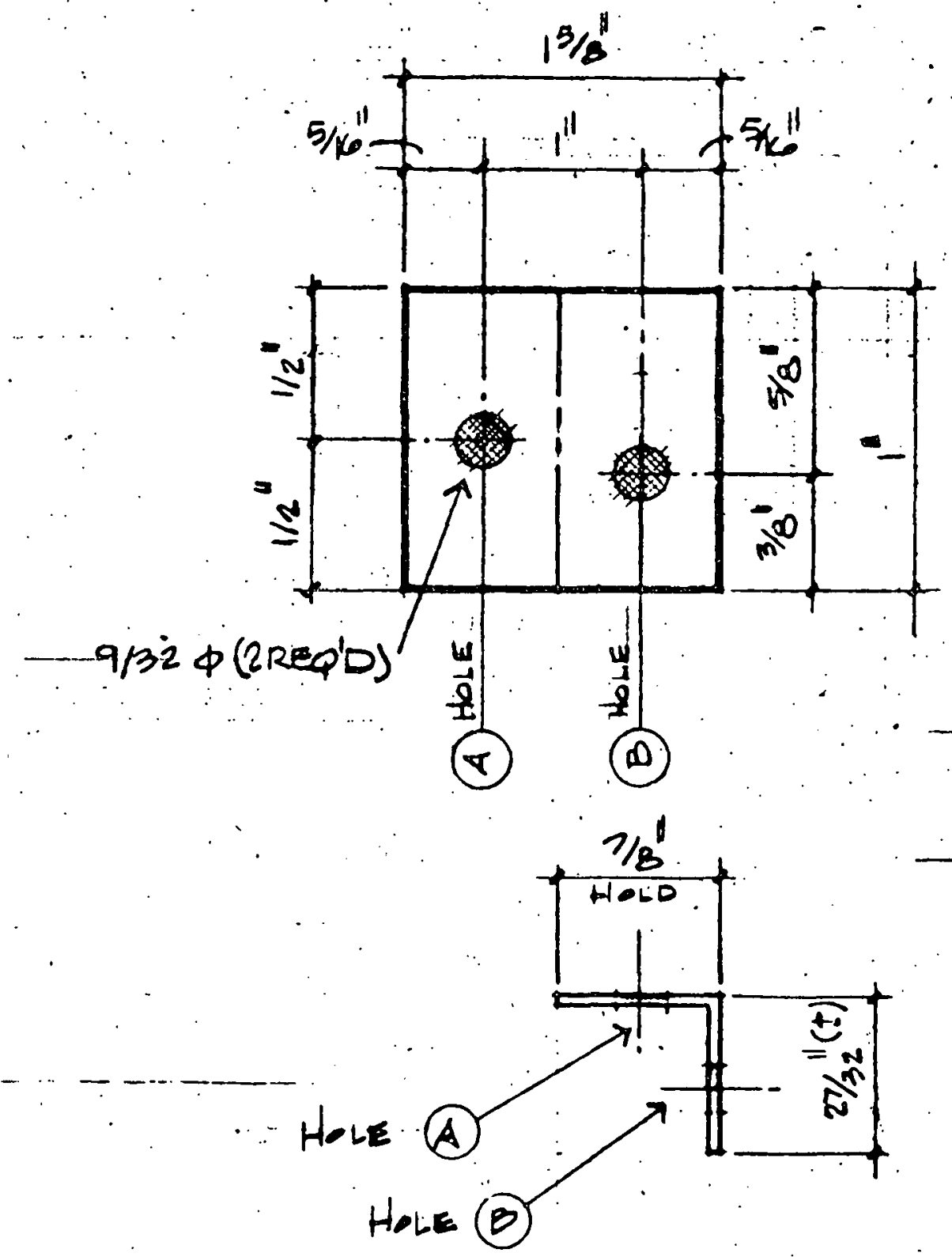

MATERIAL: 16 GA. GIT GALV. (01-01-001) SHEAR GIZE $\left|S_{B} \times\right|\left(.01 \mathrm{ft}^{2}\right)$ ALL DENDS $90^{\circ}, .016$

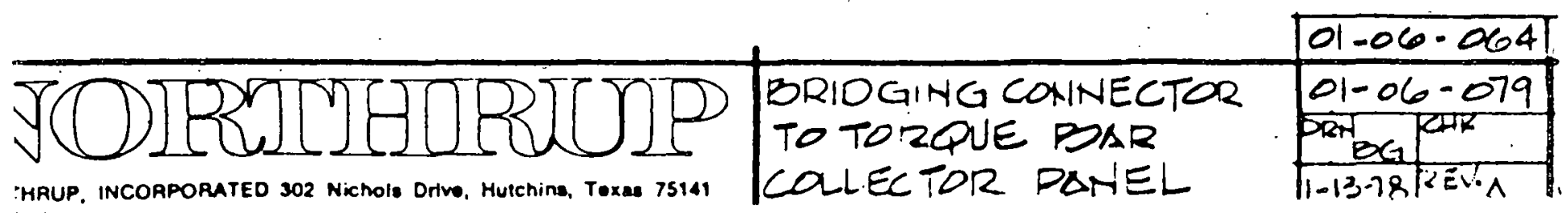


(d) $01,11-008, n^{\circ} 8$ ser $25 w, 70$ 20ec

(Y) O1- 17 -OST, BAT $114 \times 21 / 4,4$ REO

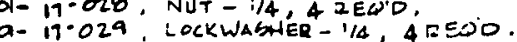

O. 11-058, hEACER DOLT- $1 / 4 \times 1 \% 1 \%, 4$ REO's.

(Z) 01-17-045, PIN - 1/4 $\times 2^{1 / 4}, 12$ eEs'o

O1- 11.056 . FXSTENER LOCK - $1 / 4,12$ REQD

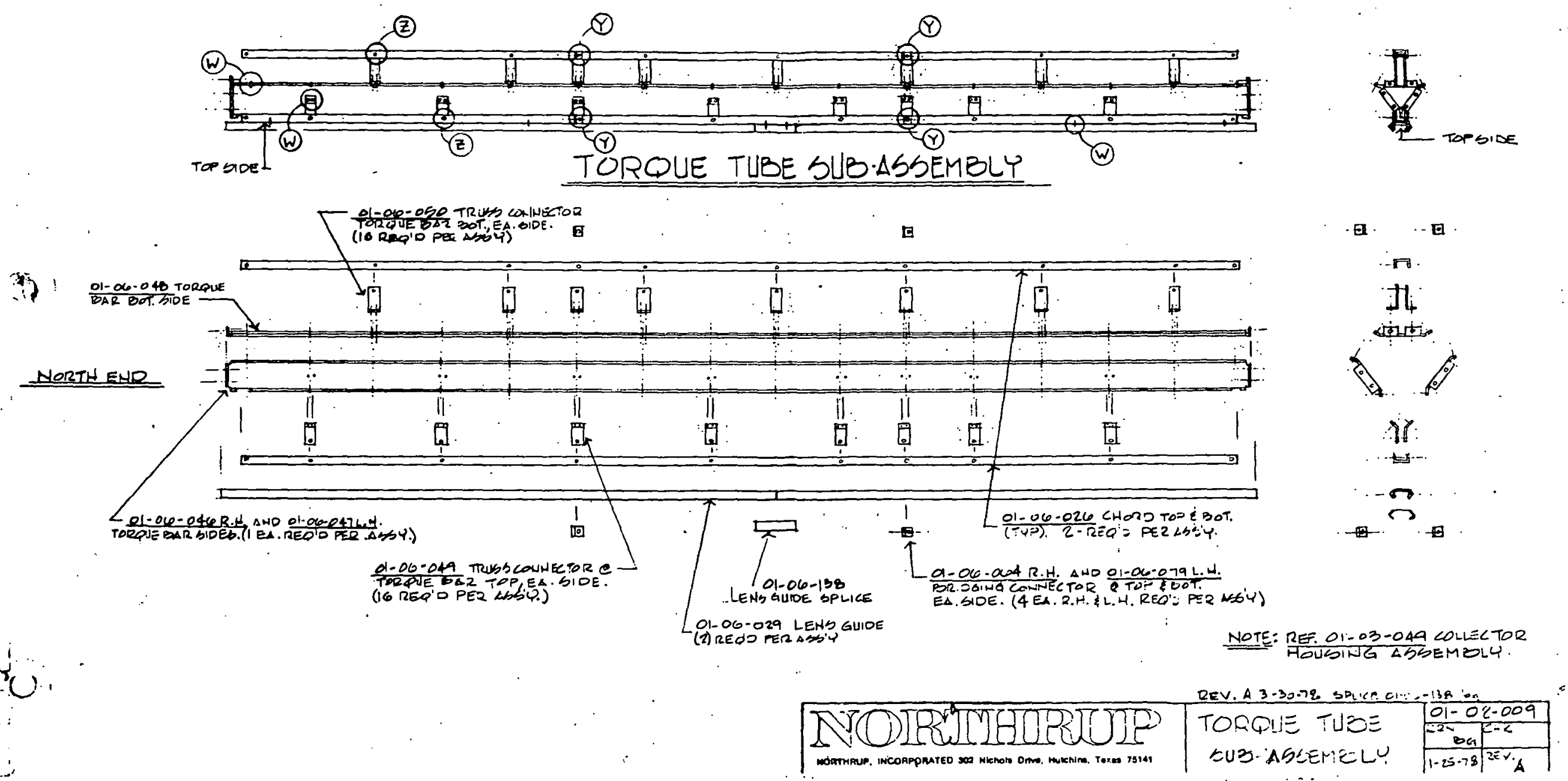



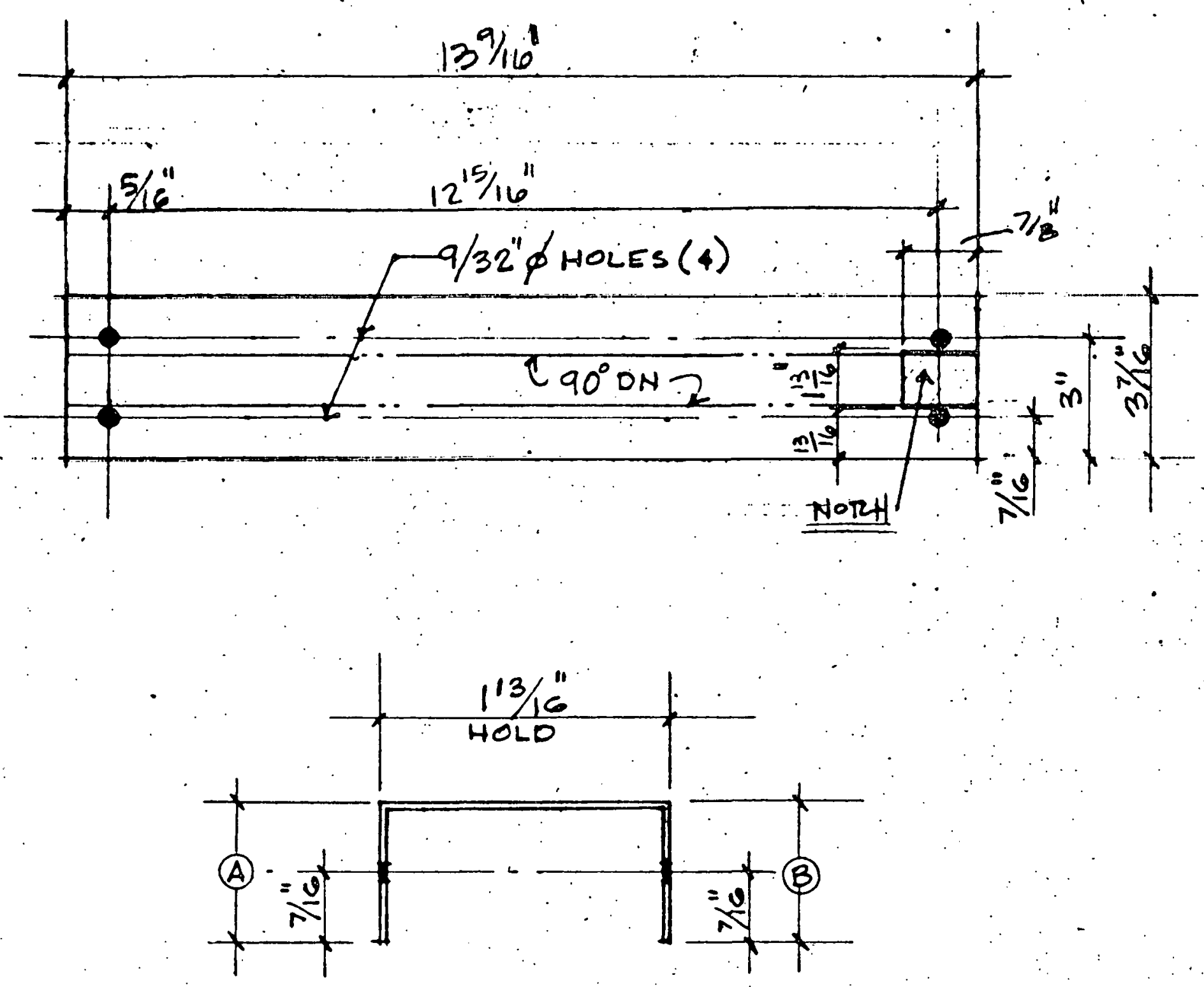

NOTE: MAKE (A) = (B) $\quad 7 / 8 " \pm$

20 GA. GALV. STL. (01-01-002)

$$
37 / 16 \times 139 / 16 \cdot 32 \cdot F T^{2}
$$

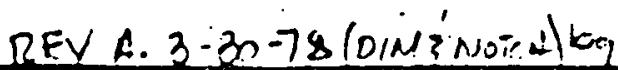

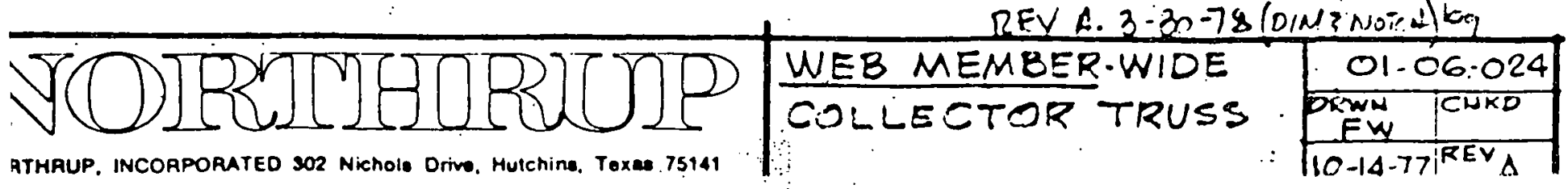

120 

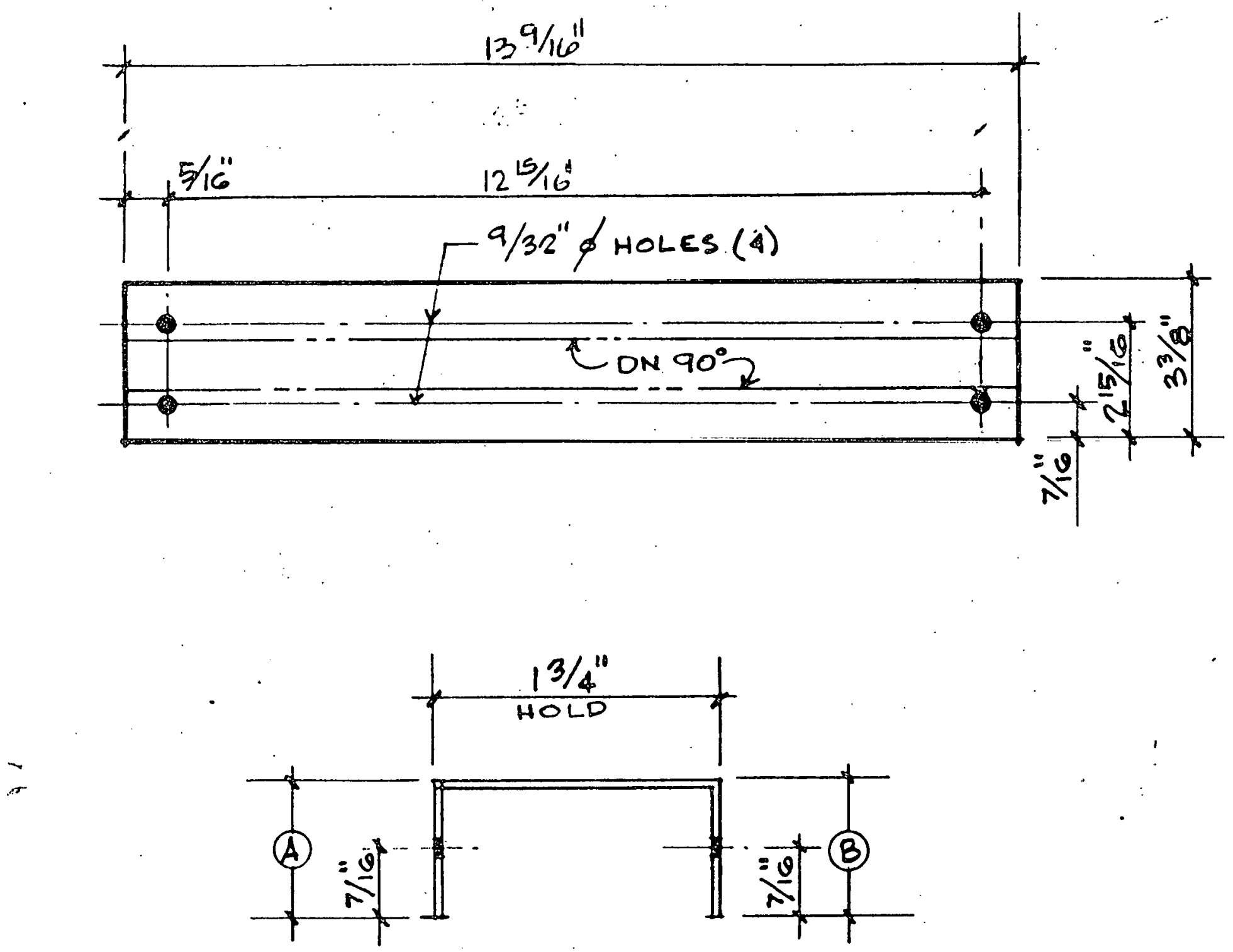

NOTE: MAKE (A) = (B) $7 / 8$ " \pm

MTL: 20 GA.GALY STL (01-01-008) .32. FT2 $33 / 8 " \times 13^{-9} / 6$

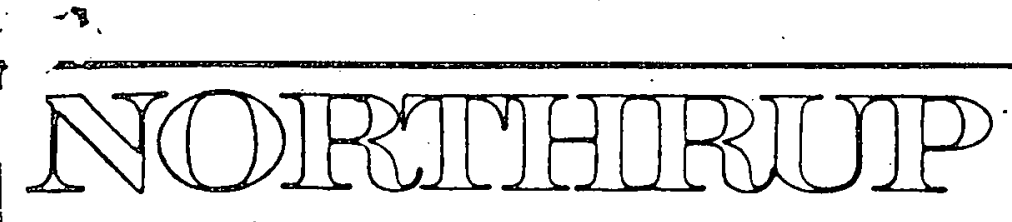

WEB MEMBER-NARRO'N COLL'TR TRUSS \begin{tabular}{|c|c|}
\hline $01-06-025$ \\
\hline DRN FW & CHR \\
\hline 10.27 .77 & REV \\
\hline
\end{tabular} 


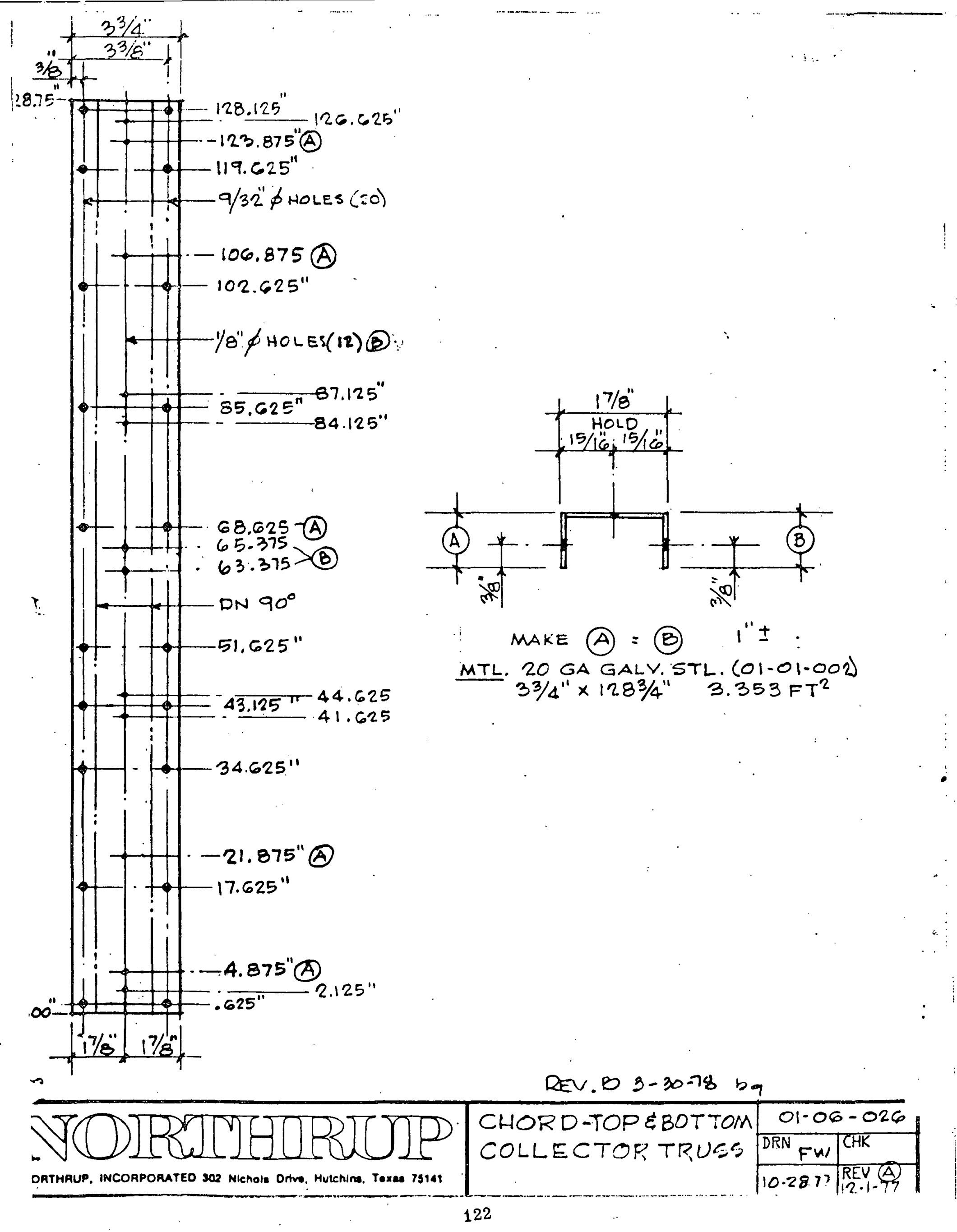




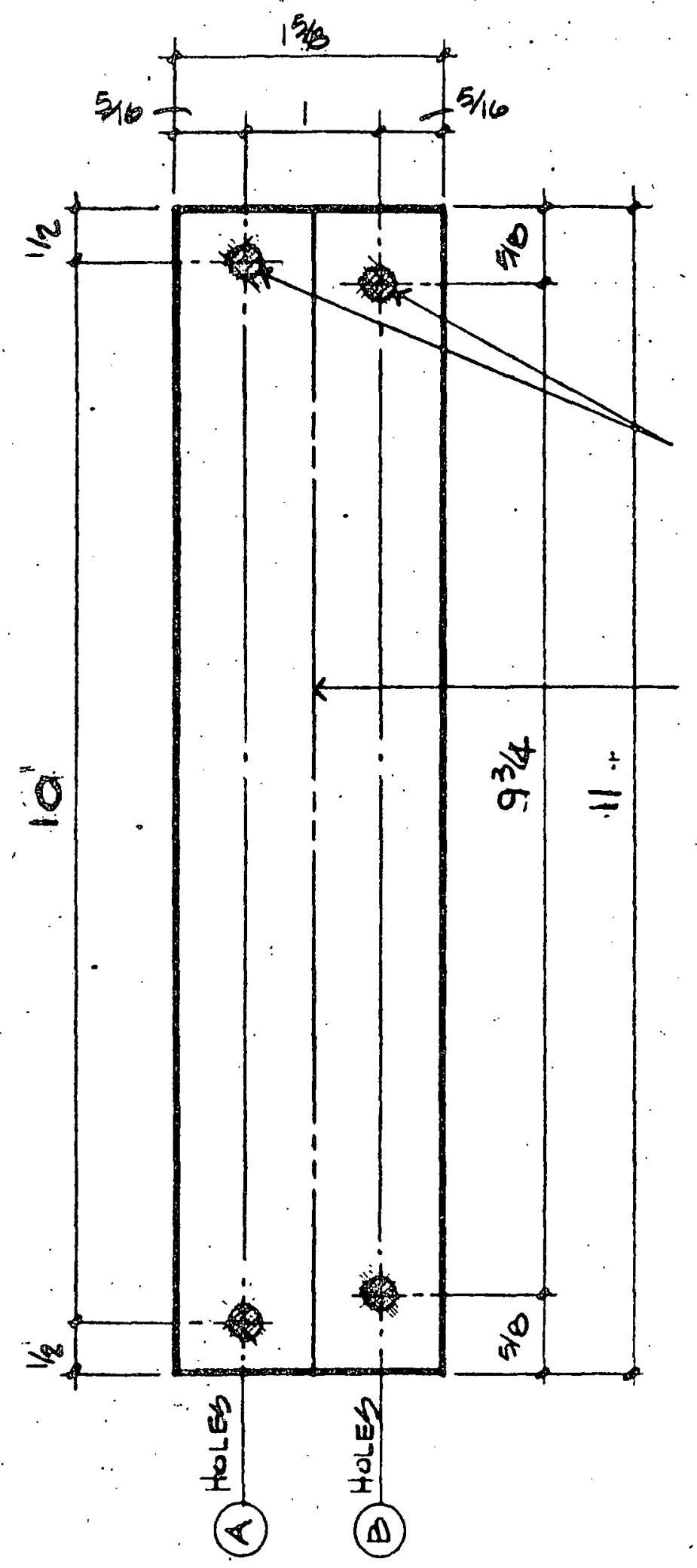

9/33 Q HOLES ( 4 REQ'O)

DN $90^{\circ}$

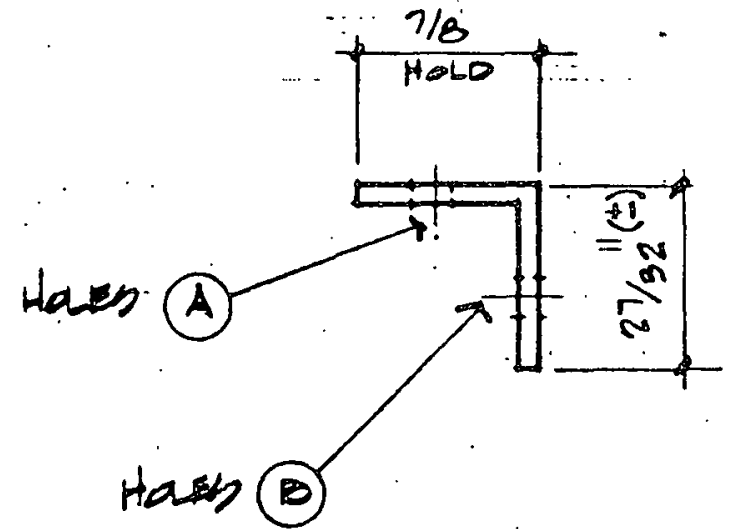

MATERISL:

BHESR BIZE $15 / \mathrm{b} \times 11:$ (D. $\left.12 \mathrm{ft}^{2}\right)$

REV. A. 3-30-78 DiM. by.

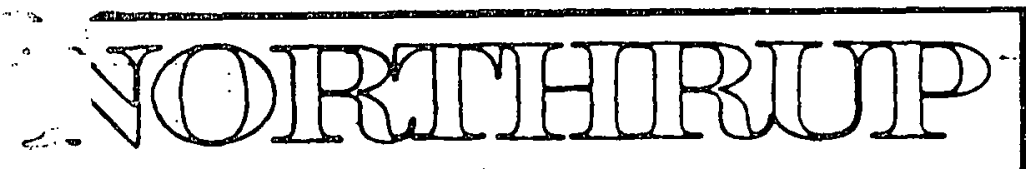

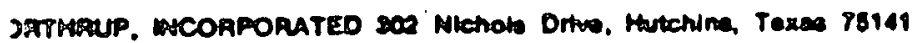
BRIPGING-VERTICAL COLLECTOR PANEL

\begin{tabular}{|c|c|}
\hline \multicolumn{2}{|c|}{$01-06-063$} \\
\hline DRN DSG & CHK \\
\hline $1-13-73$ & REV $A$ \\
\hline
\end{tabular}



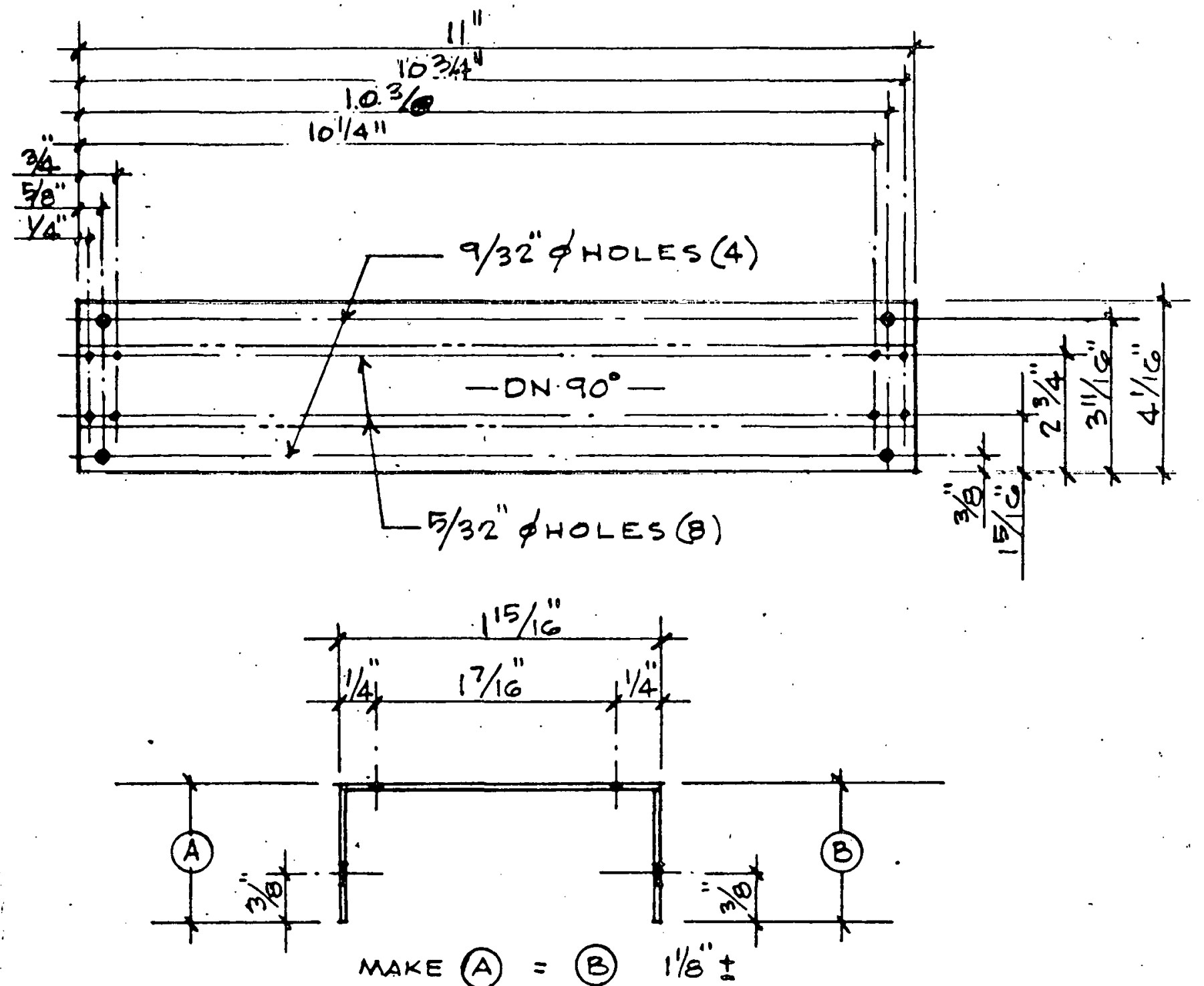

MTL: 20 GA.GALV STEEL (01.01-002).

$$
4 \% 16^{11} \times 11^{1.3 . F^{2}}
$$

REY.A $3-30-79$ DM.. 'P.

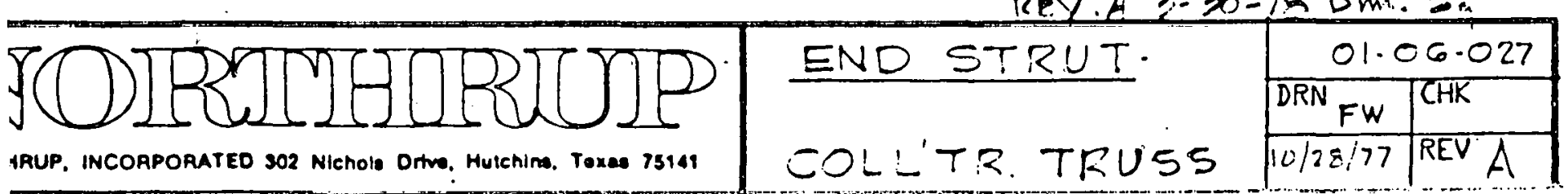




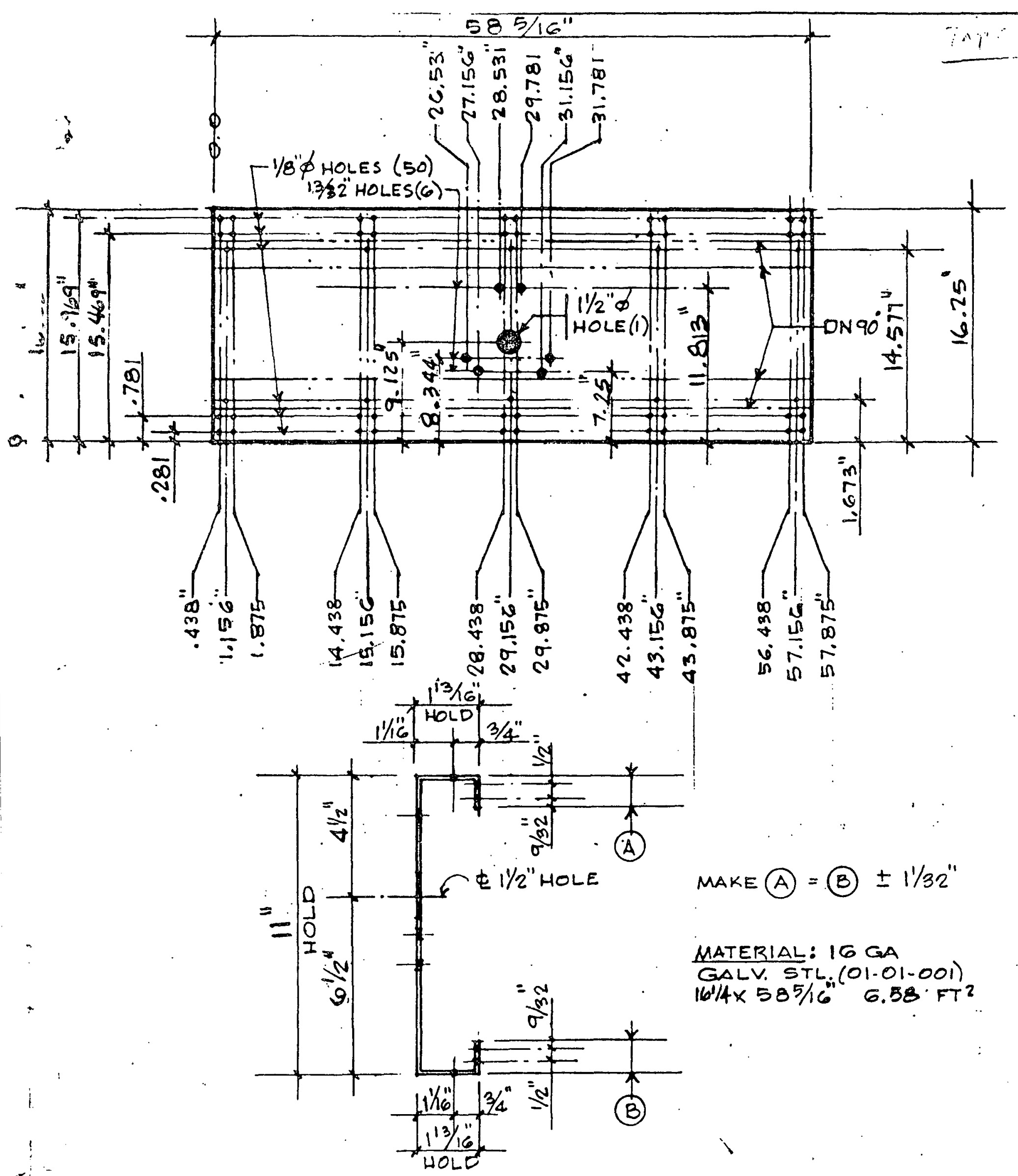

REV.A. GFP. 3-29-7869 


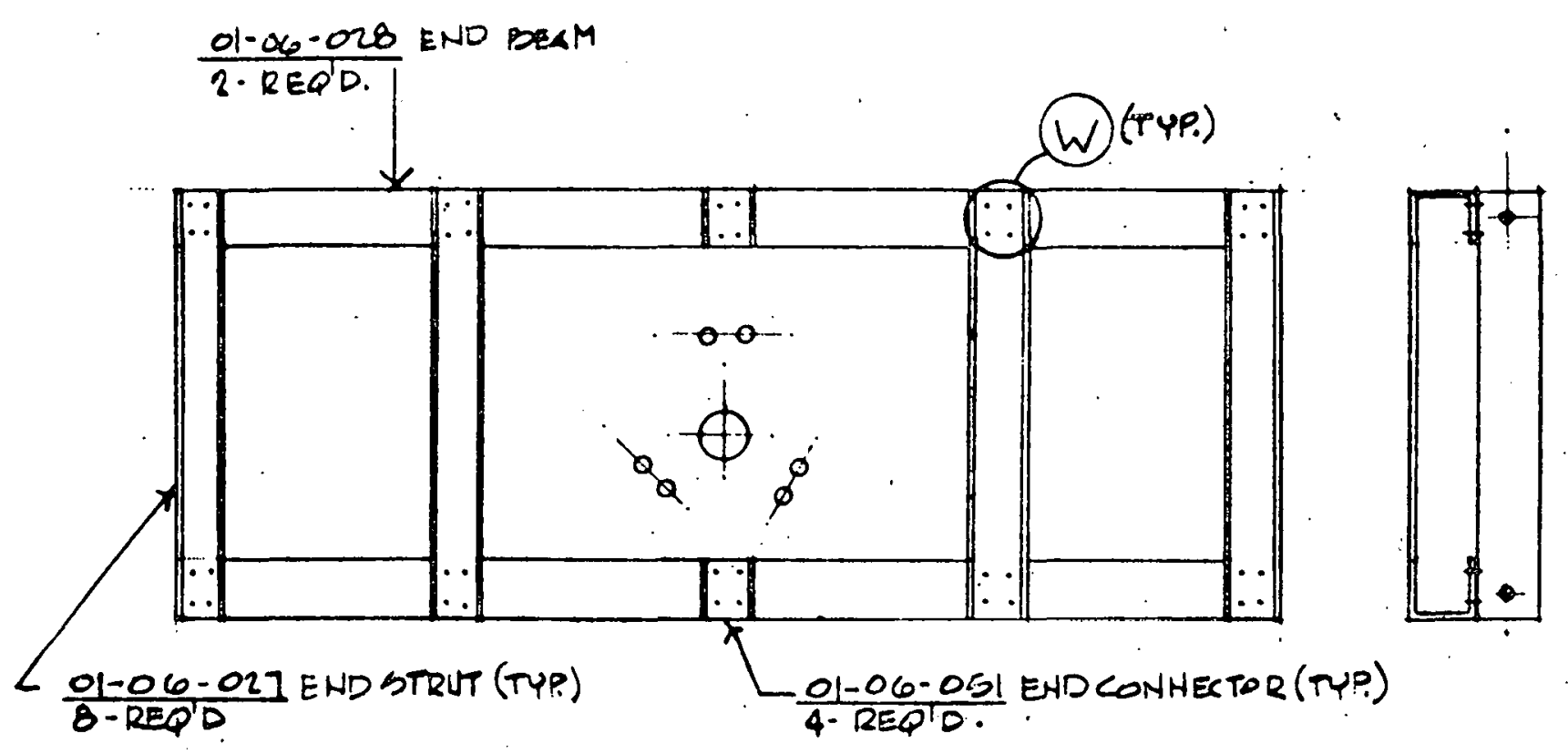

ENO BESM SUB $\triangle$ SSEMBLY

COMMON PARTS-

(W) al-O17-DOB, No o DEREW, 80 REQ'D.
NOTE: REF: O1-03-049 (COLLECTOR HOIDING ASOY LOCATION)

is.

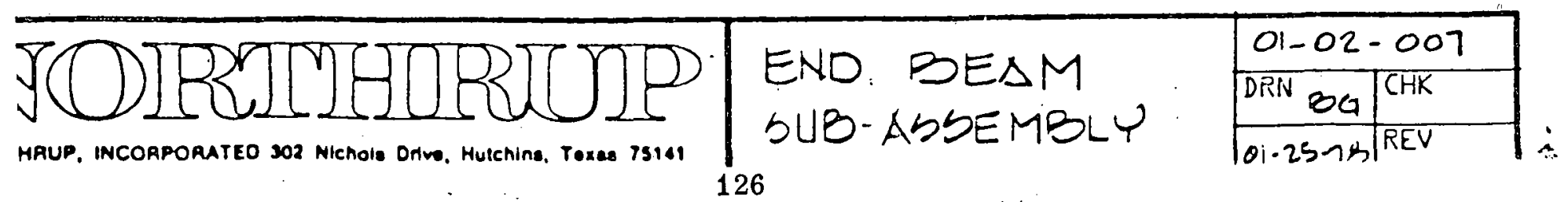



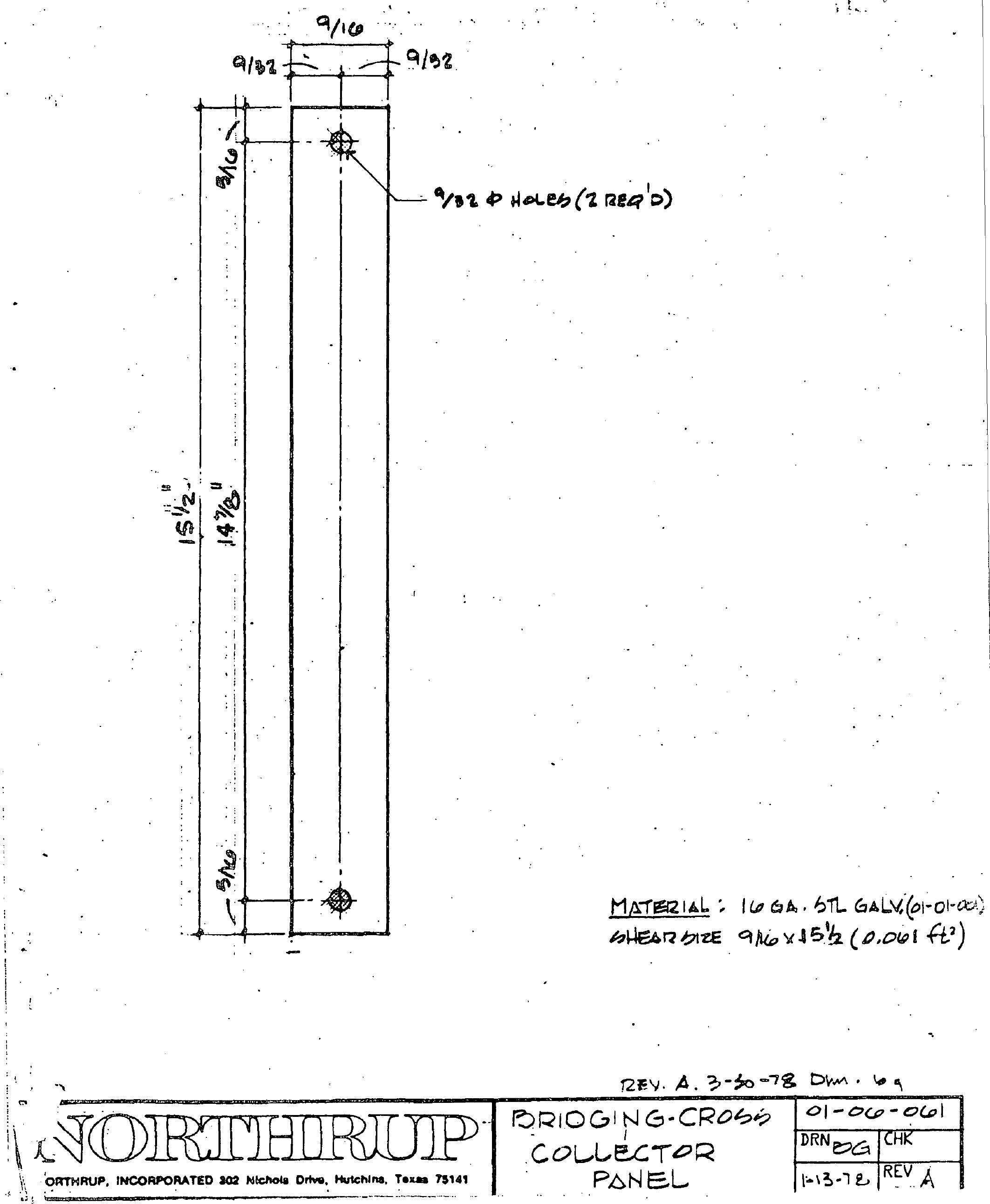

127. 

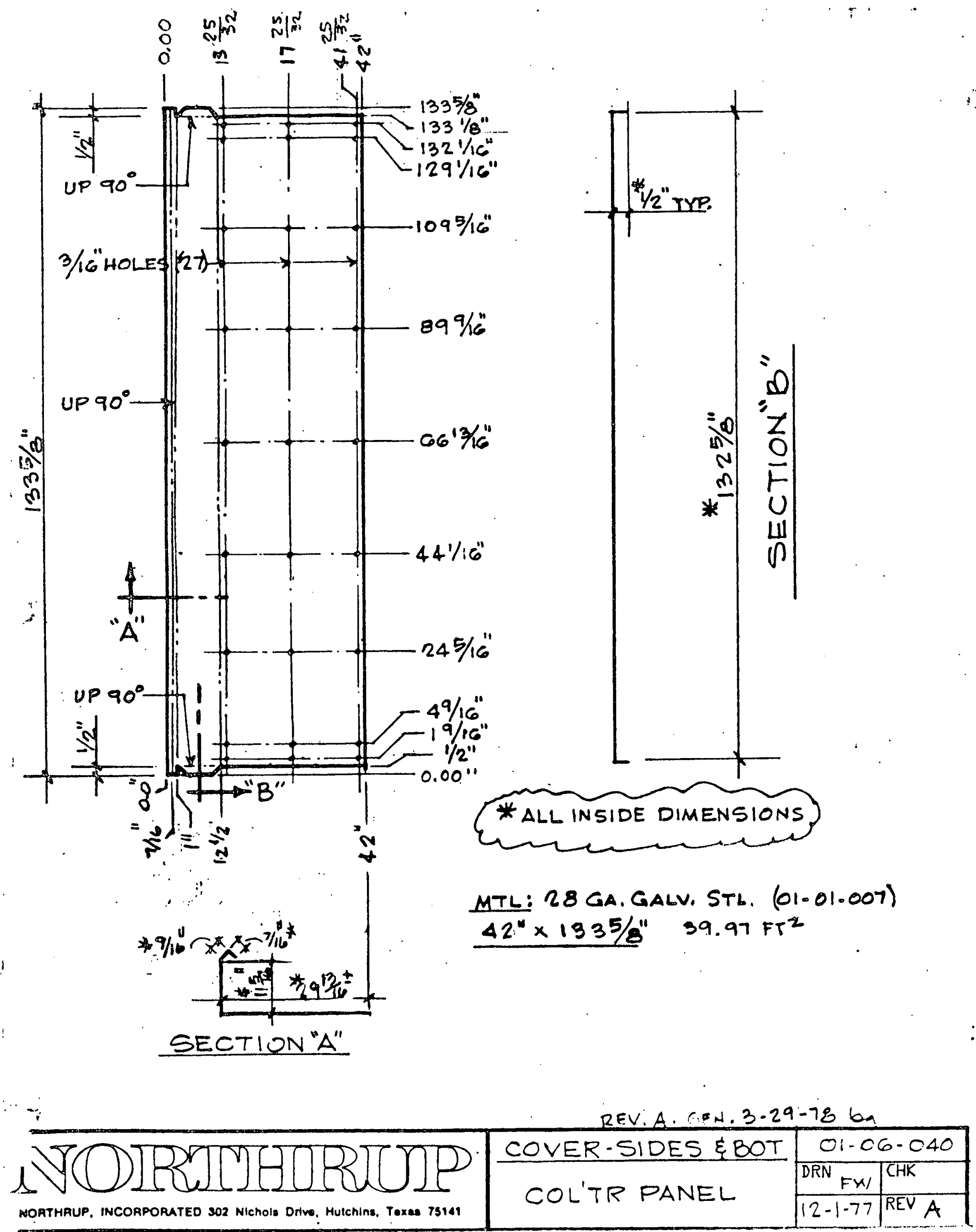


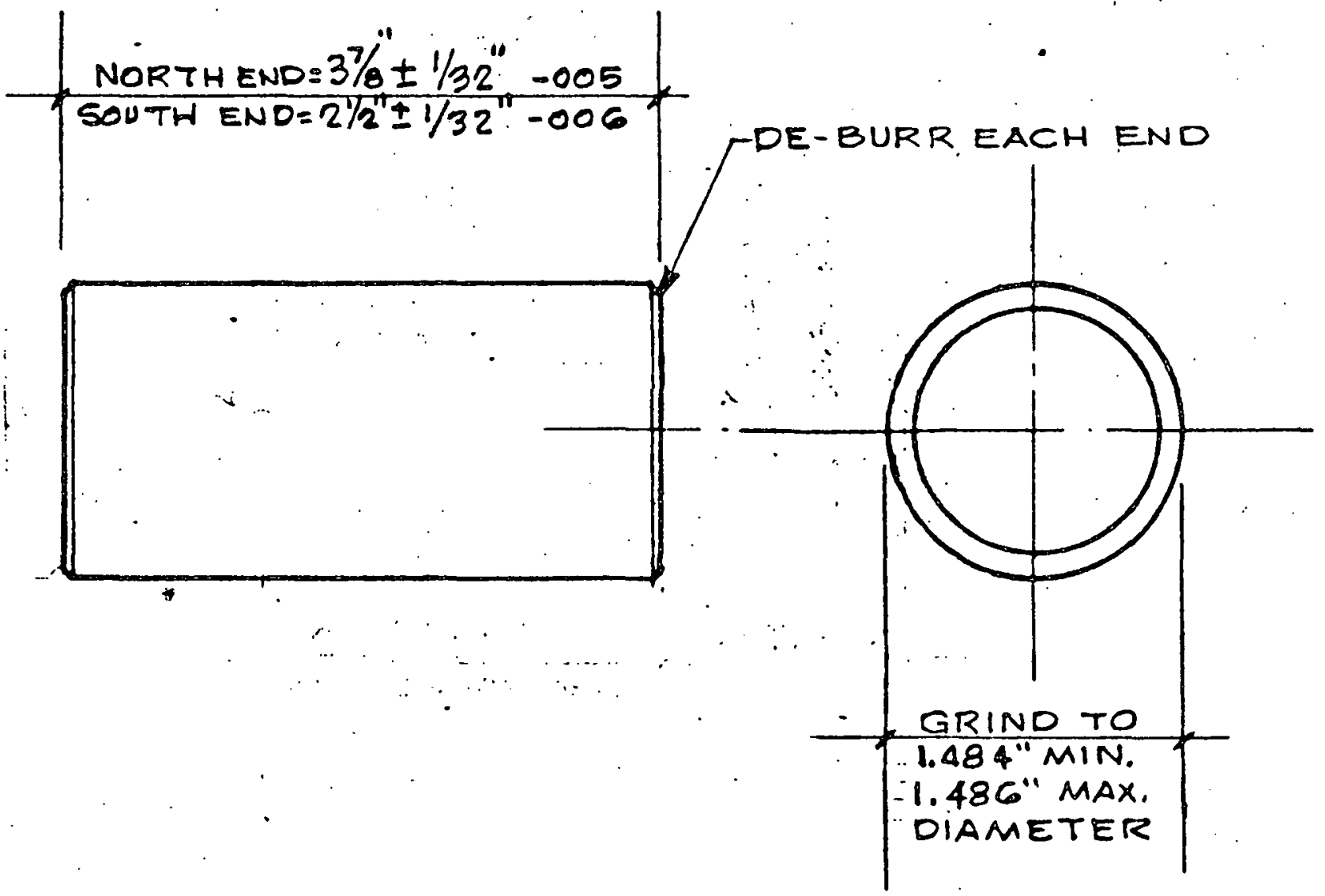

MATERIAL: COLD DRAW, SEAMLESS, STEEL TUBING, ASTM A.5IG, MECH. GRADE (P/N OI.01.605) $1 \% / 2$ O.0. K $0.109^{\prime \prime W A L L T H K . ~}$

FABRICATION: GRIND TO INDICATED O.D. WITH CENTERLESS GRINOER, CUT TO LENGTH ON LATHE OR BY SAWING. DEBURR EACHEND. DRILL PULLEY SET HOLE IN NORTH END,.005 ONLY.

OI- $18-005$ NORTHEND 01.18 .006 SOUTH ENO

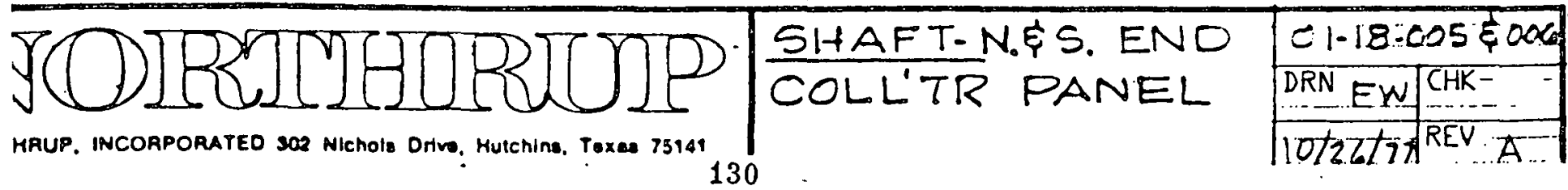




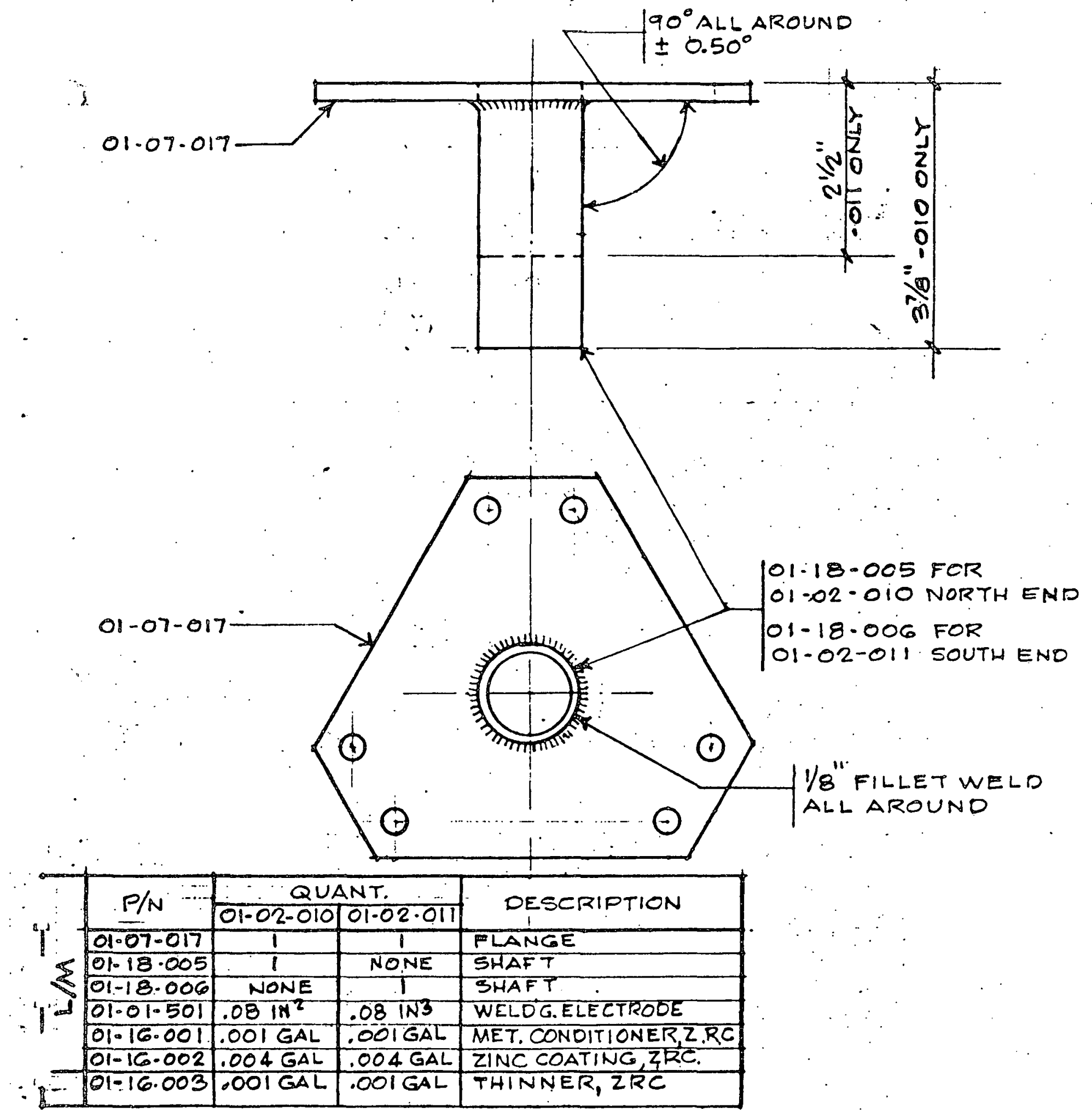

-ARRICATION EFINISH: WELO PER AWS EAISC. REMOVE LOOSE RUST AND LUUX WITH POWER STEEL EUFFER. CLEAN SURFACES WITH MET. CONDITIONER PER OI-16-001. COAT ALL SURFACES WITH ZINC COATING (.002"TO.0O3"THK) AND CURE TER O1-16-002 \& 01-i6-003. STORE, HANDLE. E EHIP IN SUCH MANNER AS TC FREIENT DENTS NICKS, FLUX. OR DAMAGE TO -UTSIDE SIRFACE OF EHAFT.

O1-O2. OHO NORTH END O1:02-0I1 SOUTH END

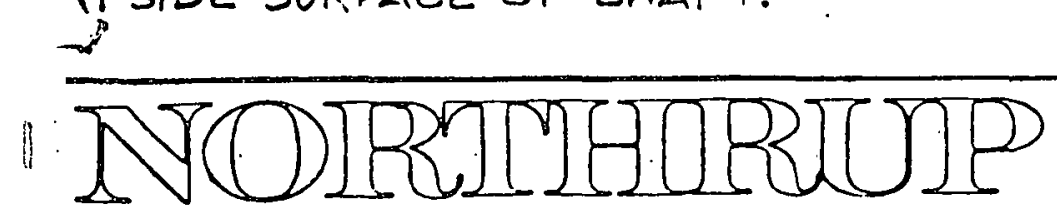

NORTMRUP. INCORPORATED 302 NiChols Drive. MUtChins, TEXas 75141
FLANGED SHAFT ASSBY. O1-C2-010 \$011 NORTH \& SOUTH ENID - COLLTR PANEL

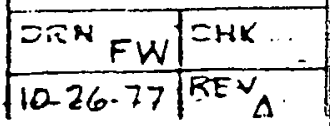




\section{Z.R.C. ${ }^{\Sigma}$ METAL CONDITIONER}

- DE-OXIDIZES - NEUTRALIZES RUST

- DE-GREASES - REMOVES GREASES \& OILS

- PHOSPHATIZES - FOR MAXIMUM ADHESION

3 STEPS IN ONE EASY CPERATION...

Z.R.C. Metal Conditioner is a new concept in metal surface preparation. Although formulated as a metal pretreatment for Z.R.C. Cold Galvanizing Compound, it is equally effective to pre-clean and prepare any type of metal surface (ferrous or non-ferrous) for any type of painting.

In ore easy operation 2.R.C. li.eial Consitioner will:

1. Neutralize rust oxides on any ferrous or non-ferrous metal surface. Because of these de-oxidizing properties, it also acts as an excellent brightener for aluminum, zinc and copper surfaces (including aluminum truck bodies).
2. Removes residual oils and greases by its unique detergent action

3. Slightly etch the metal surface and leave a phosphate deposit that will maximise the adhesion properties of any top-coat.

As an additional convenience and cost saving feature... Z.R.C. Metal Conditioner needs no rinsing or neutralizing - simply...

(A) AFPLY (B) ALLOW TO SET 5 MINUUTES (C) WIPE the surlace is now pre-treated and ready for top-coating

\section{APPEARANCE Blue liquid (very slight solvent odor) \\ PACKAGING Gallons-Packed 6 Polyethylene jugs per case (shipping weight 53 lbs. per case) Quarts - Packed 12 Polyethylene bottles per case (shipping weight 27 lbs. per case) \\ Z.R C Metal Conditioner meets ond exceeds the requirements of foderel Specification MIL-C-10578 (Type II)}

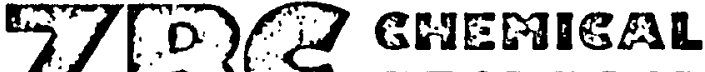 Cinn N $\infty$}

21 NEWPOAT AVE., OUINCY, MASS. 02171 TEL. 617/328.6700

Z.R.C. is a liquid compound containing $95 \%$ zinc in the dried film, that fuses to clean iron, steel or aluminum through electrochemical action to provide maximum protection against rust and rust creepage.

\section{Z.R.C. vs. PAINT}

Il is important that there is an understanding of the conceptual difference beween Z.R.C. and paint.

While all types of paints provide only a "skin" prolection, Z.R.C. imparts cathodic action against corrosion - i.e., in the presence of moisture its metallic zinc content becomes an anode and the underlying surface a cathode. Corrosion attacks the zinc leaving the base metal untouched.

If the "skin" of a paint is broken, in any way, it will rust in that area and also allow rust creepage under the break. However, if Z.R.C. is scratched to the bare metal the zinc will form an insoluble zinc salt on the exposed area. This heals the break and continues to protect the surface against any corrosion.

Thus - Z.R.C. will not allow rust creepage.

Color: Matte flat light gray.

Packaging: $3-1 / 2$ gallon pails, gallons, quarts, half-pints and aerosol cans.

- One package - No premixing - Unlimiled pot-life - Unlimited shelf-life - Ready to apply
APPLICATION METHODS - COVERAGE APPLICATION. Z.R.C. may be applied by brush, spray, dip or roller.

SPRAY (Low pressure compressor type). Atomizing pressure 50 p.s.i. Orifice of tip 0.080 ( $8 \% 1 \ldots$ (1) ths) \pm 5 . Viscosity - Reduce Z.R.C. in ratio of 4 parts Z.R.C. to 1 part $X X X$ Thinner*.

SPRAY (Airless type). Pressure 650-750 p.s.i. Orifice of tip 0.013-0.023 (13/1000 to 23/1 (1,0).

$0.018(18 / 1000)$ generally delivers optimum performance. Viscosity - No reduction required.

BRUSH OR ROLLER - No reduction required. Apply as received from container.

DIPPING - Reduce Z.R.C. in ratio of 3 parts Z.R.C. to 1 part XXX Thinner".

DRYING - When Z.R.C. is air dried, it is set to touch in 20-30 minutes and may be re-coated in 12 hours.

CURING - Z.R.C. requires curing before being exposed to severe service. When air-dried, it cures progressivcly up to 14 days. Curing may be accelerated to a matter of minutes if heat is applied. Suggested cycle for full cure is $300^{\circ} \mathrm{F}$ for 30 minutes.

FLASH POINT - Z.R.C. has a safe llash point of $104^{\circ} \mathrm{F}$ and thereby enables its use freely around welding operations.

COVERAGE - 400-500 square feet per gallon. An average brush coat of Z.R.C. will deposit $1.5-2.0$ mils (dry film thickness). This is generally sufficient for "regular" environment exposure. Two coats are suggested for severe exposure conditions.

\section{$01-16-002$ AND 01-16-003}

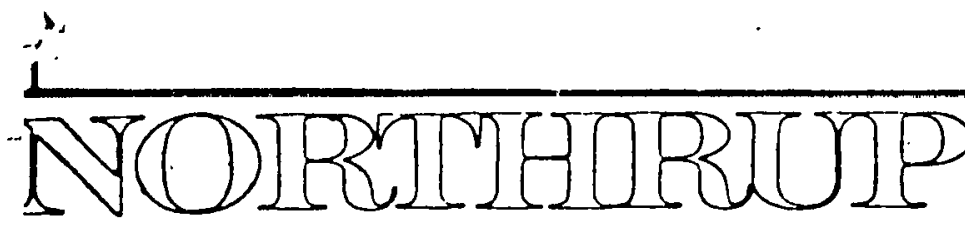

NORTMAUP. INCORPORATED 302 Nlehol, Drive. Hutchins, Texes 75141

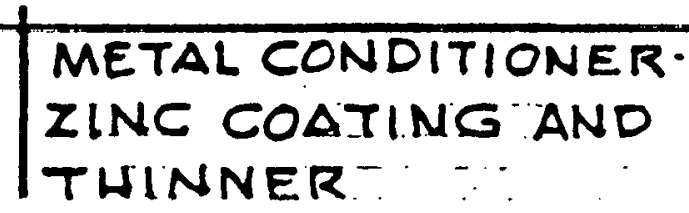




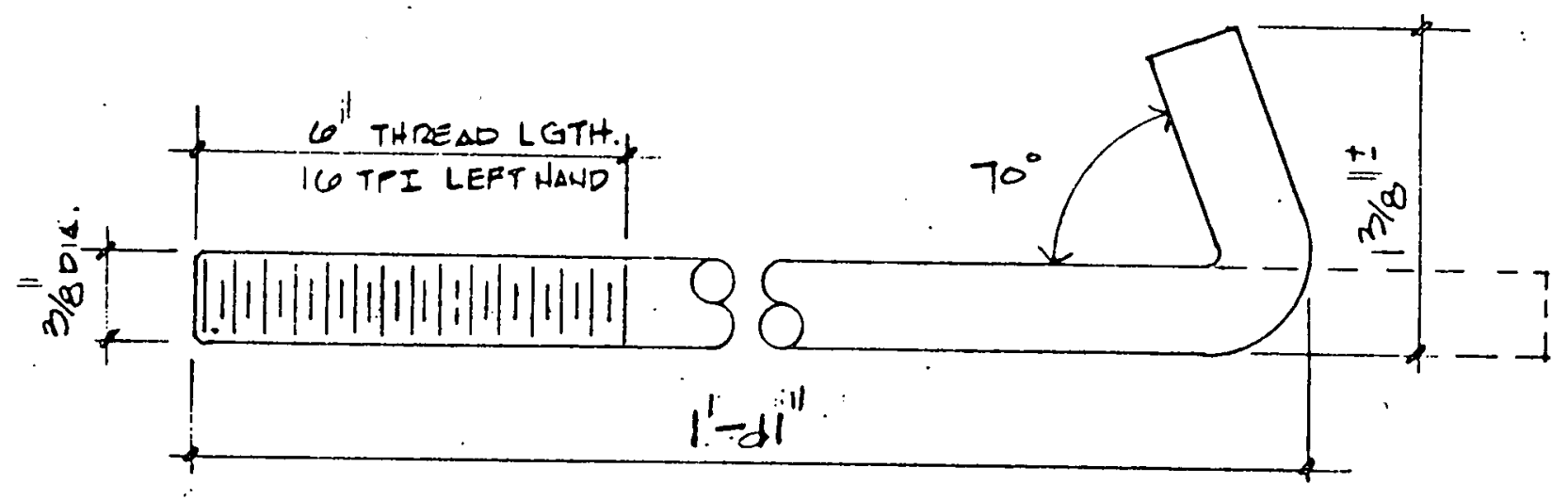

- Ne: Hotol GabV. (-125 oz a SPELTER PER/FT' ${ }^{2}$ ) AFTER FABRILATION.

-

MSTERILL= 3/8"D.4. A360 STL. ROD LENGTH PQIU:: TO DENID 2! -0! (C0.01-012)

REF. 01-04-005.01-04-006 \& $9-04-035$

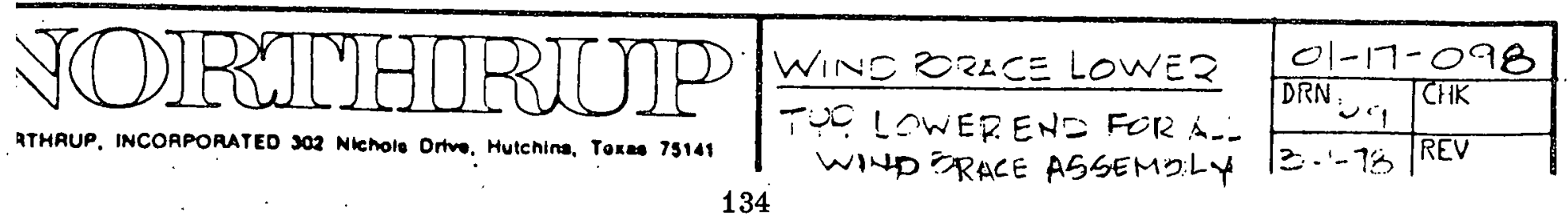




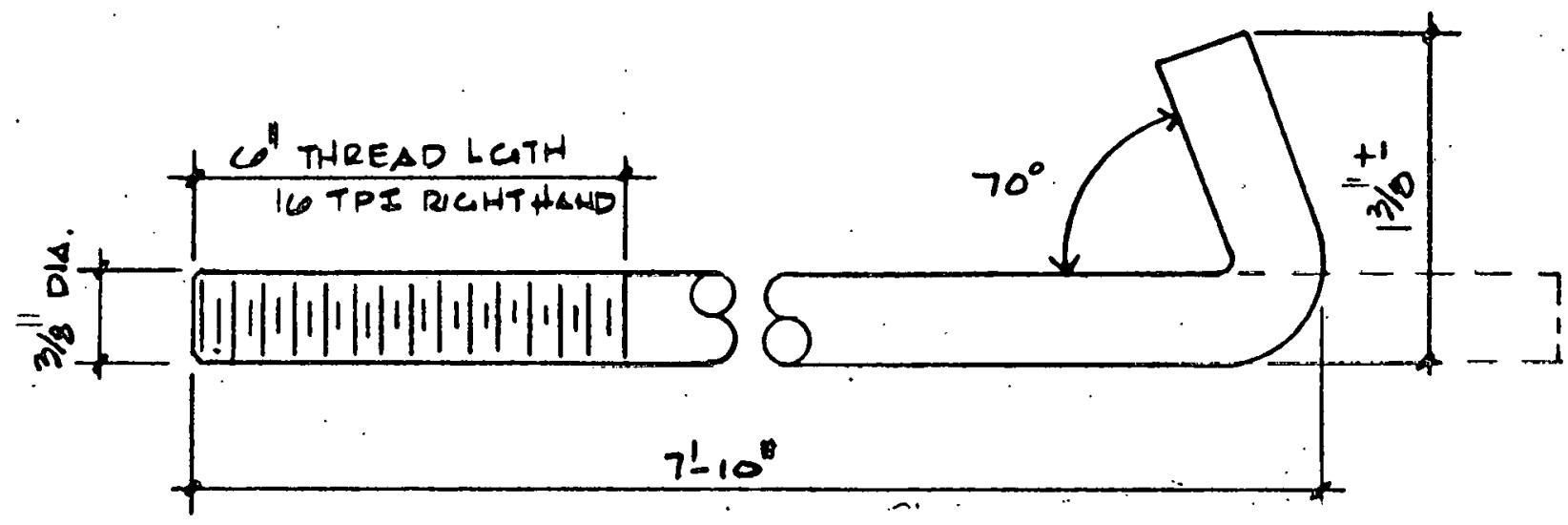

HOUE: HOT DIP GALV. (.125 OZ OF SPELTER PER/FT' $\triangle$ '

FABRICATINAN.

MATERILL: 3/BDDIA, AZ6, STL DOD.

LENGTH PRKOR T BEHD.7-11" (00-01-012)

REF.01.04-005 AND O1-04-006

NORTH ANO SOUTH (UPPER) END

WIND BRLEE.

\begin{tabular}{|c|c|}
\hline \multicolumn{2}{|c|}{$01-17-100$} \\
\hline DRN ba & CHK \\
\hline $3.1-73$ & REV \\
\hline
\end{tabular}




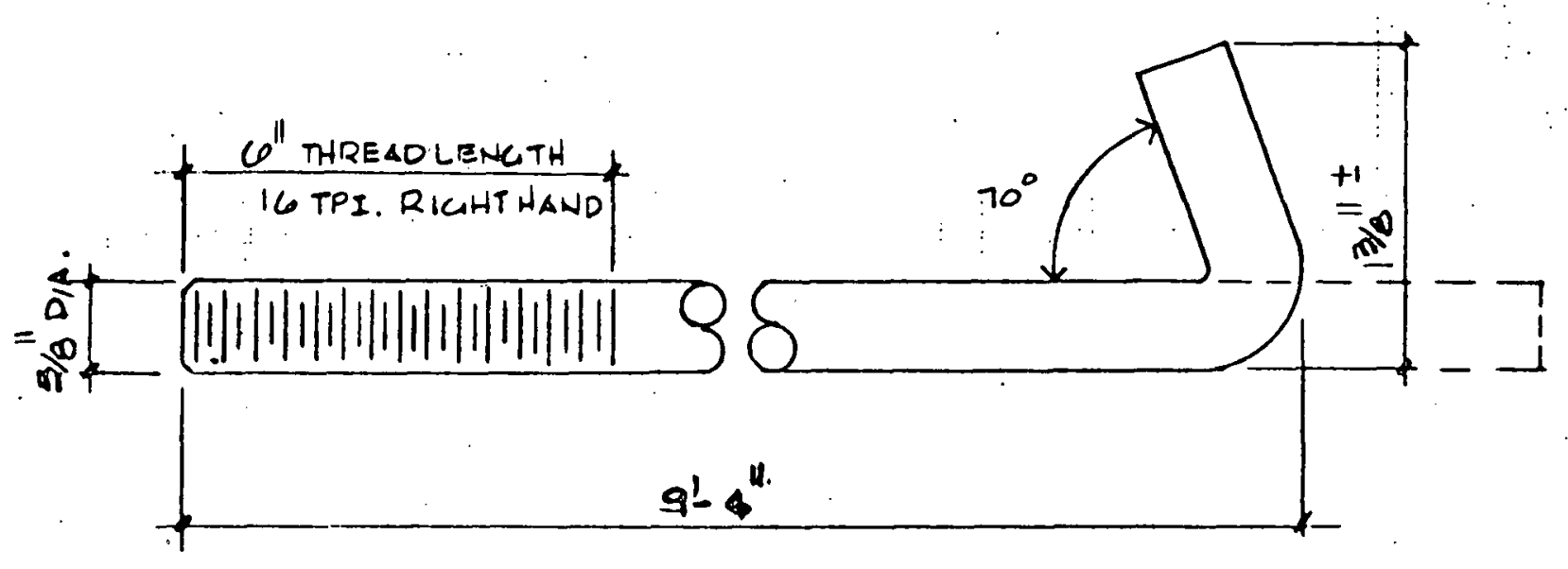

- NOTEE HOT DIP GALV. (.RSOZ.OF SPELTER PER/FT') $\triangle F$ TER

FABRTCATIUN

MSTERISL: B/8"DIA, A36, STL. ROS LENGTH PRIOR KO OEND. 9'-5" $(00.01-012)$

REF. $01-04-02,5$

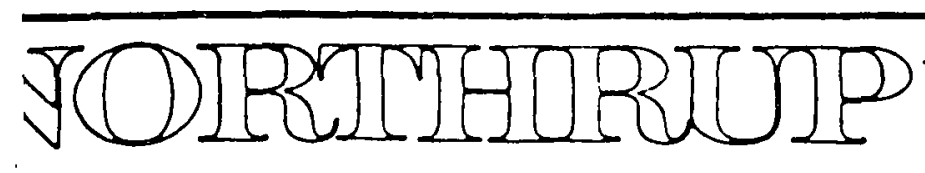

IMAUP. INCORPORATEO 302 Ntehole Otre. Hulchins, Texes 75141
NORT: (LOWER)

UEPER WINDBZZLINET.

\begin{tabular}{|c|c|}
\hline \multirow{2}{*}{\multicolumn{2}{|c|}{$0 !-17-090$}} \\
\hline & \\
\hline & \\
\hline
\end{tabular}




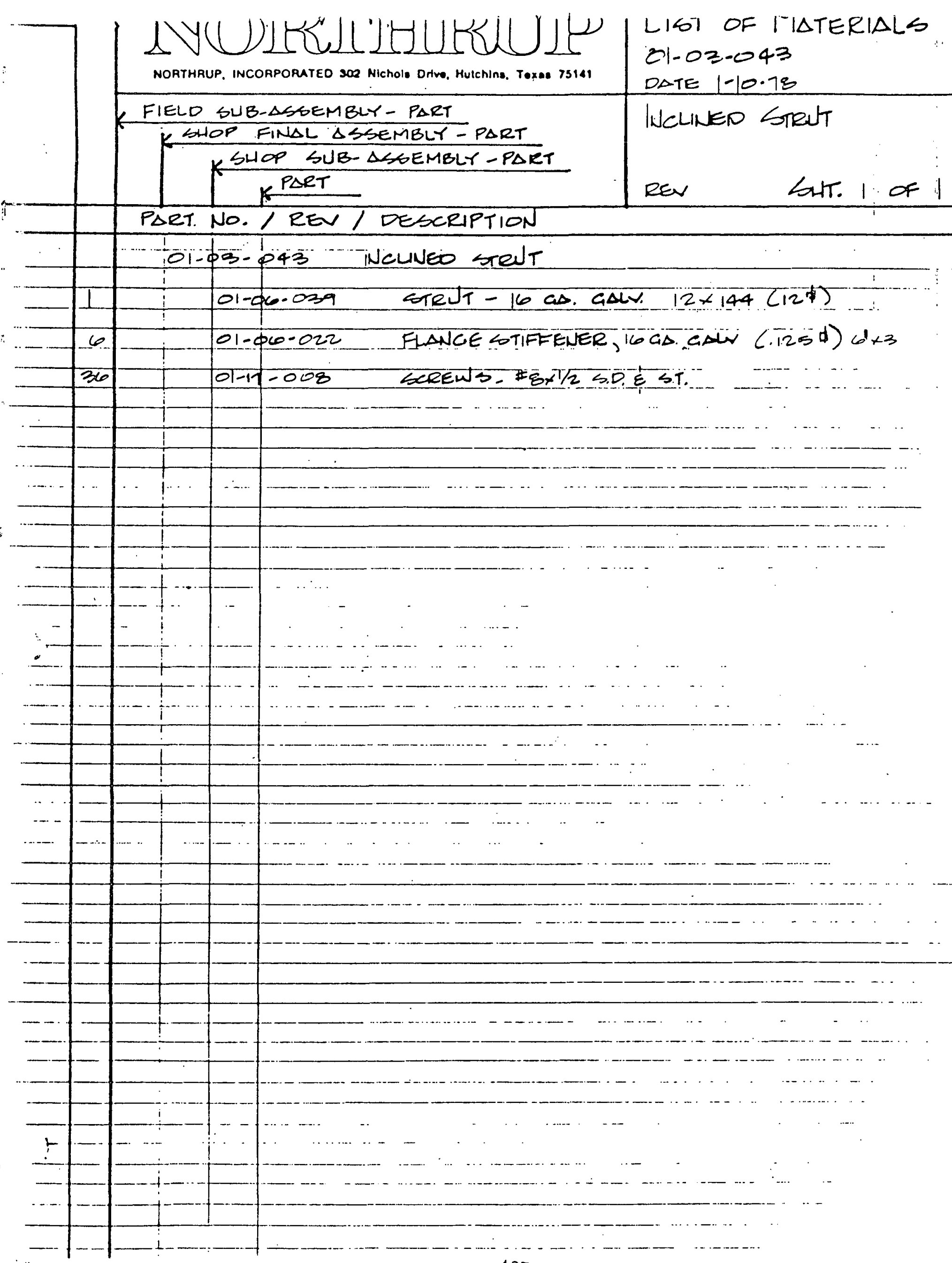



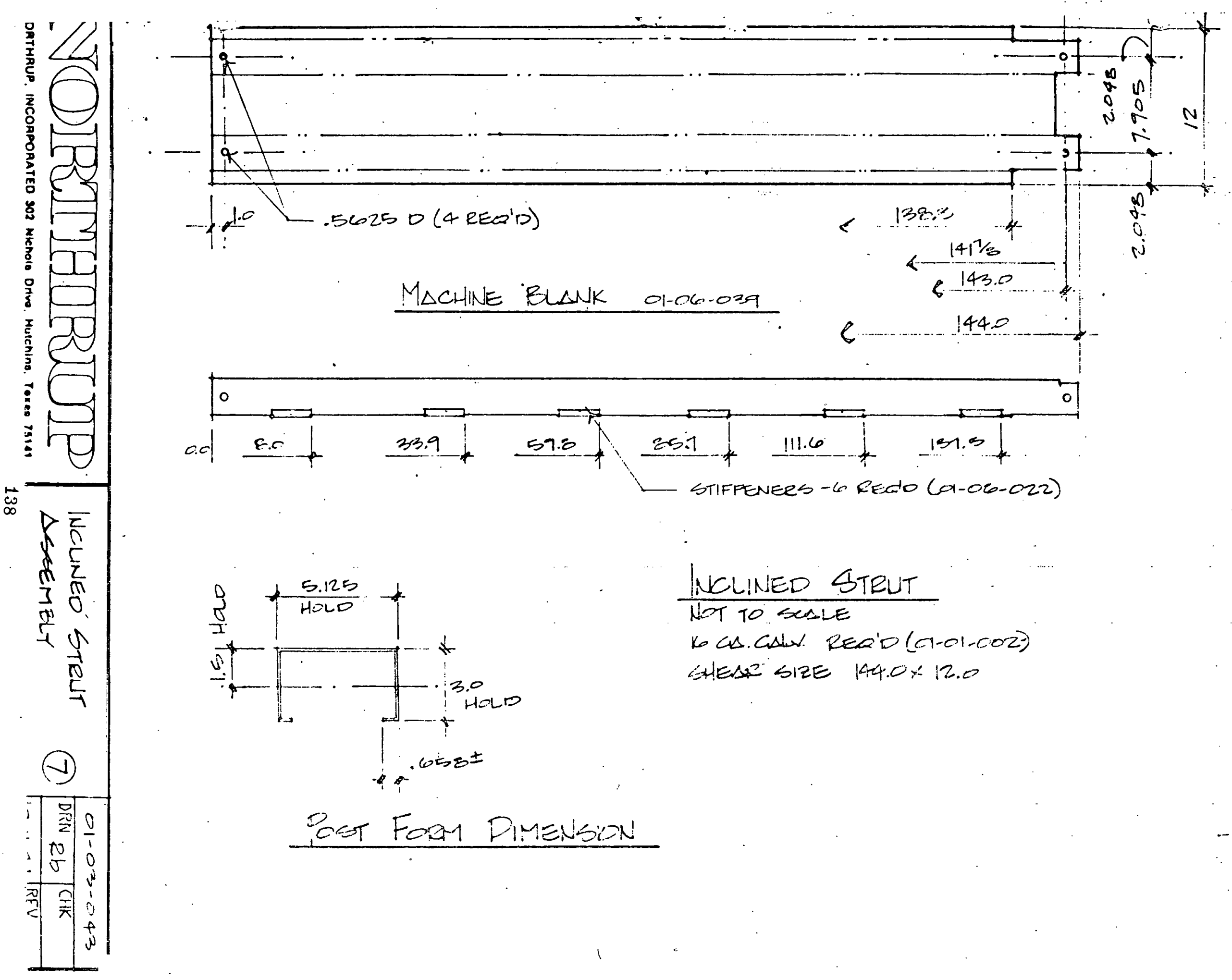

$\frac{\text { RELINED STRUT }}{\text { NOT TO SCALE }}$

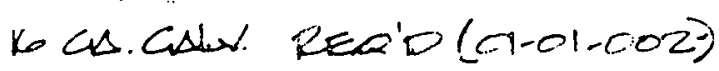
SPIES SIZE $144.0 \times 12.0$

Pest Fond DIMension 
$\therefore$ REF CNT/DRWC O1-03-043 For WORK this shlet

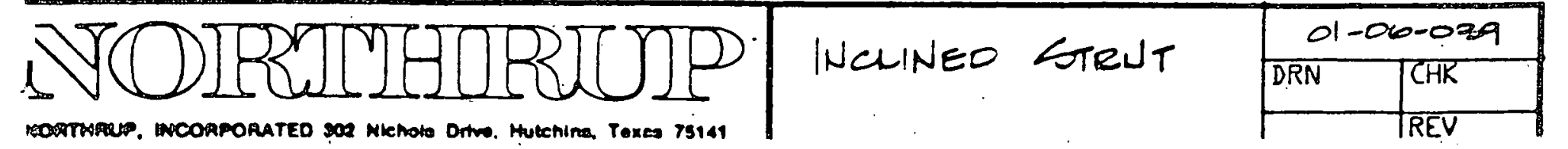




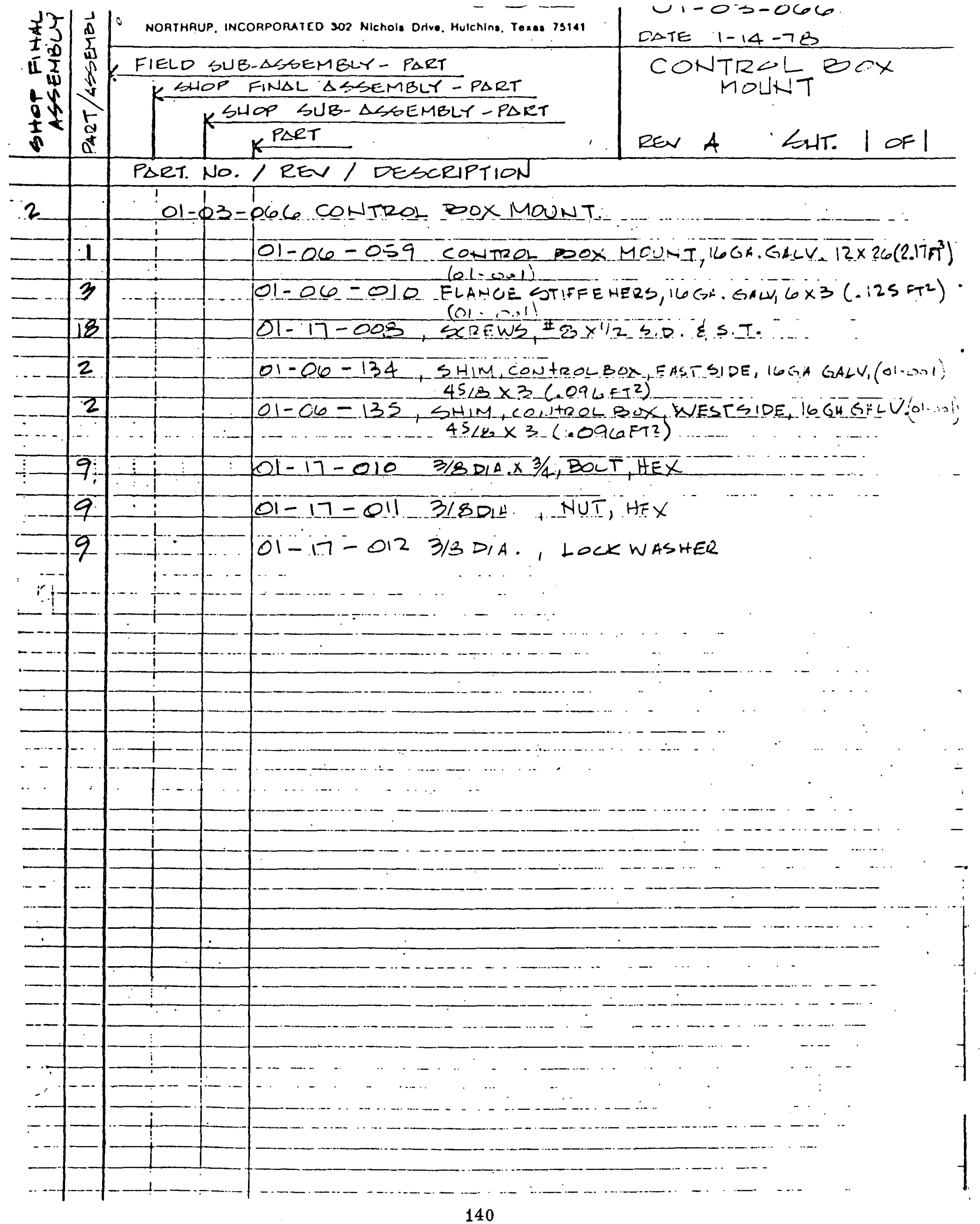



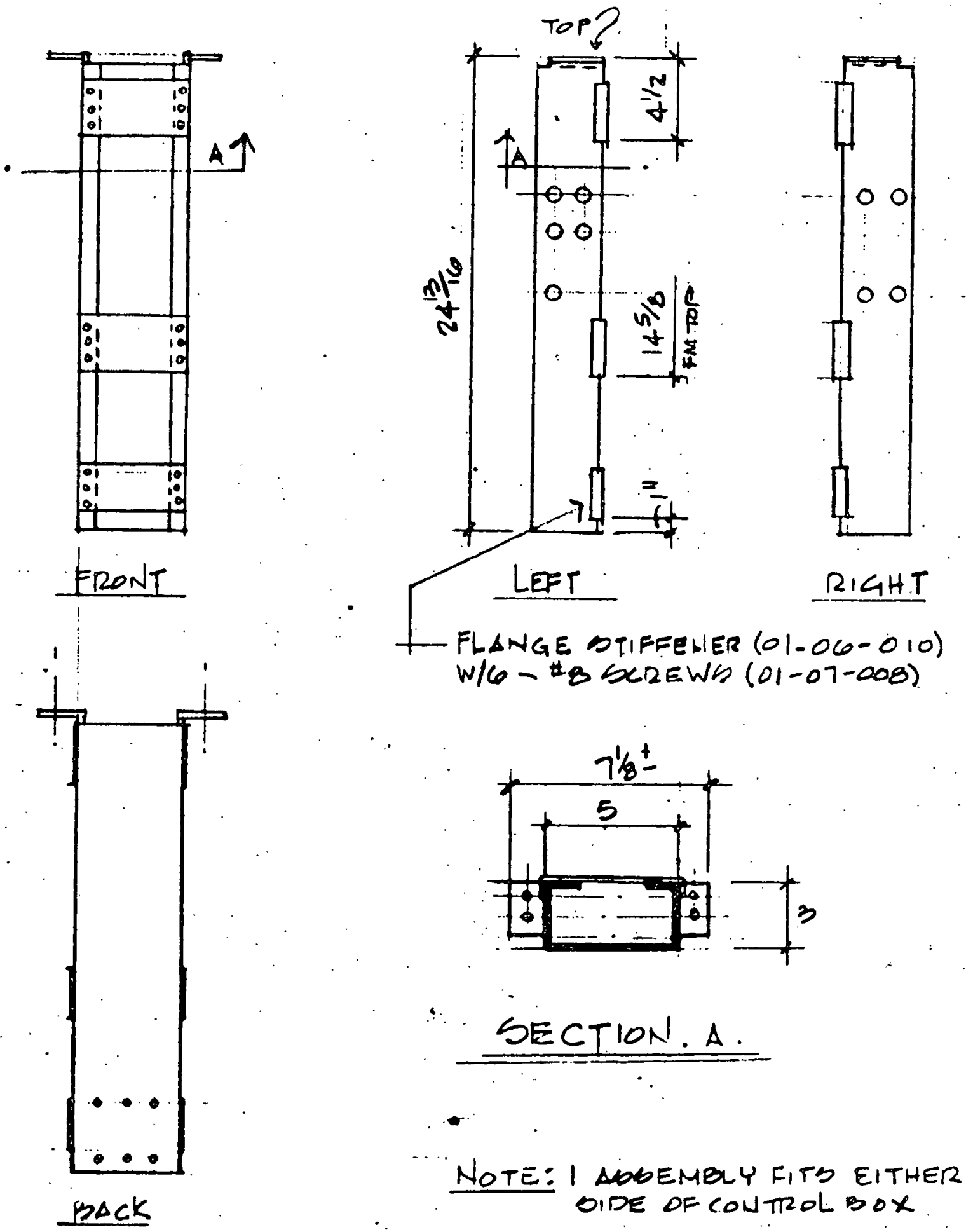

$$
\therefore 1
$$

i.

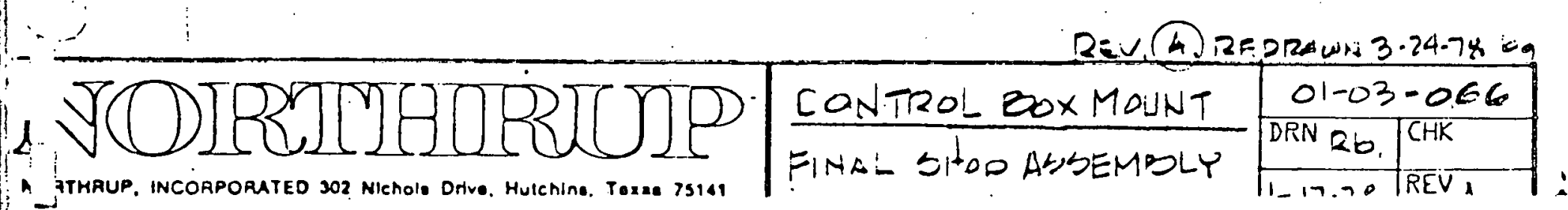




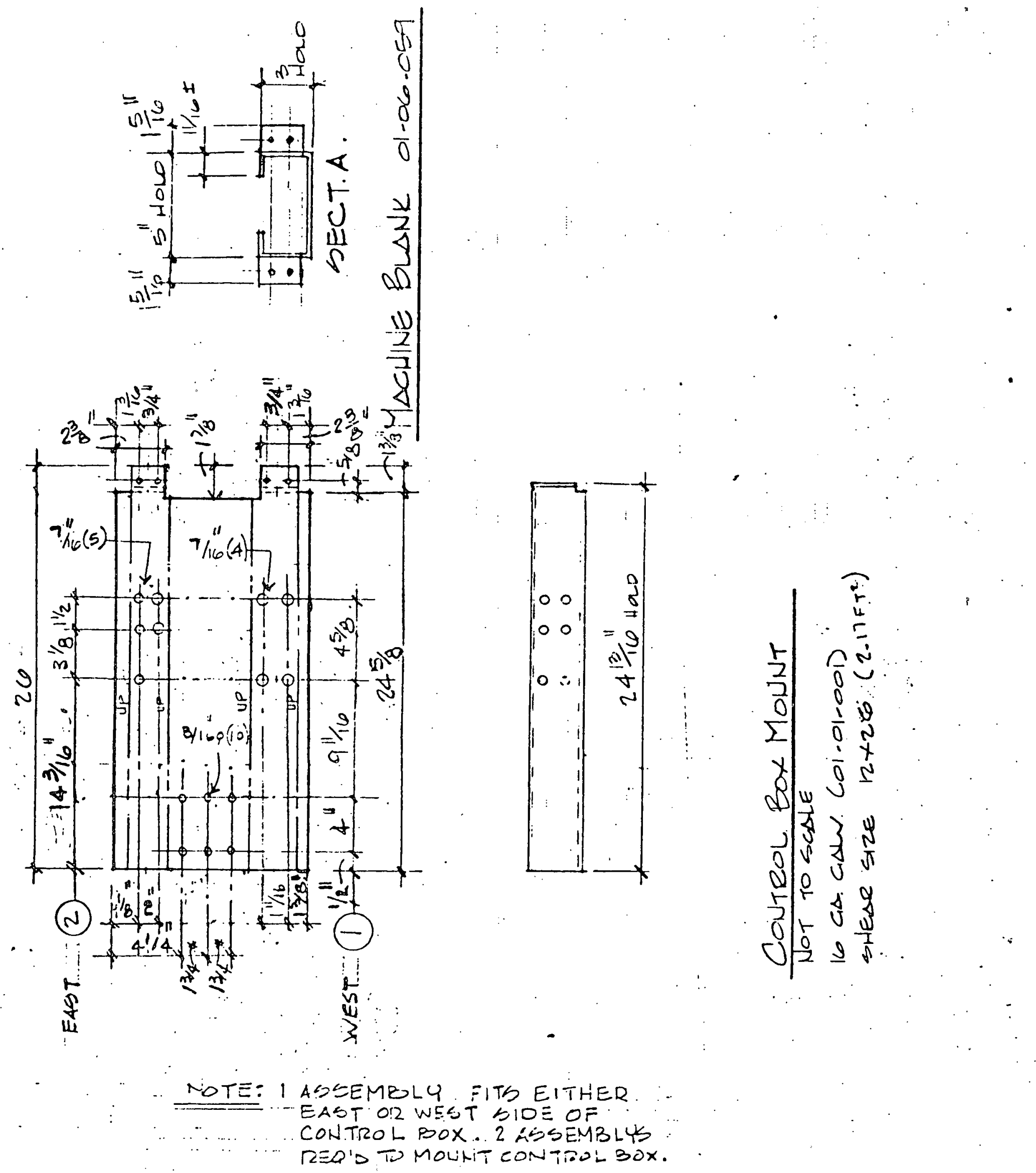

. REV. Z-24-74 i25:-

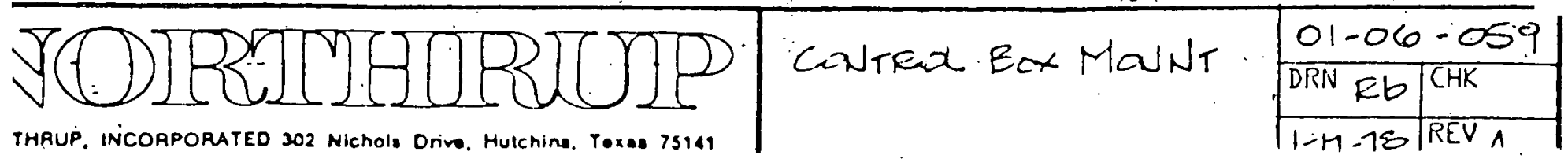




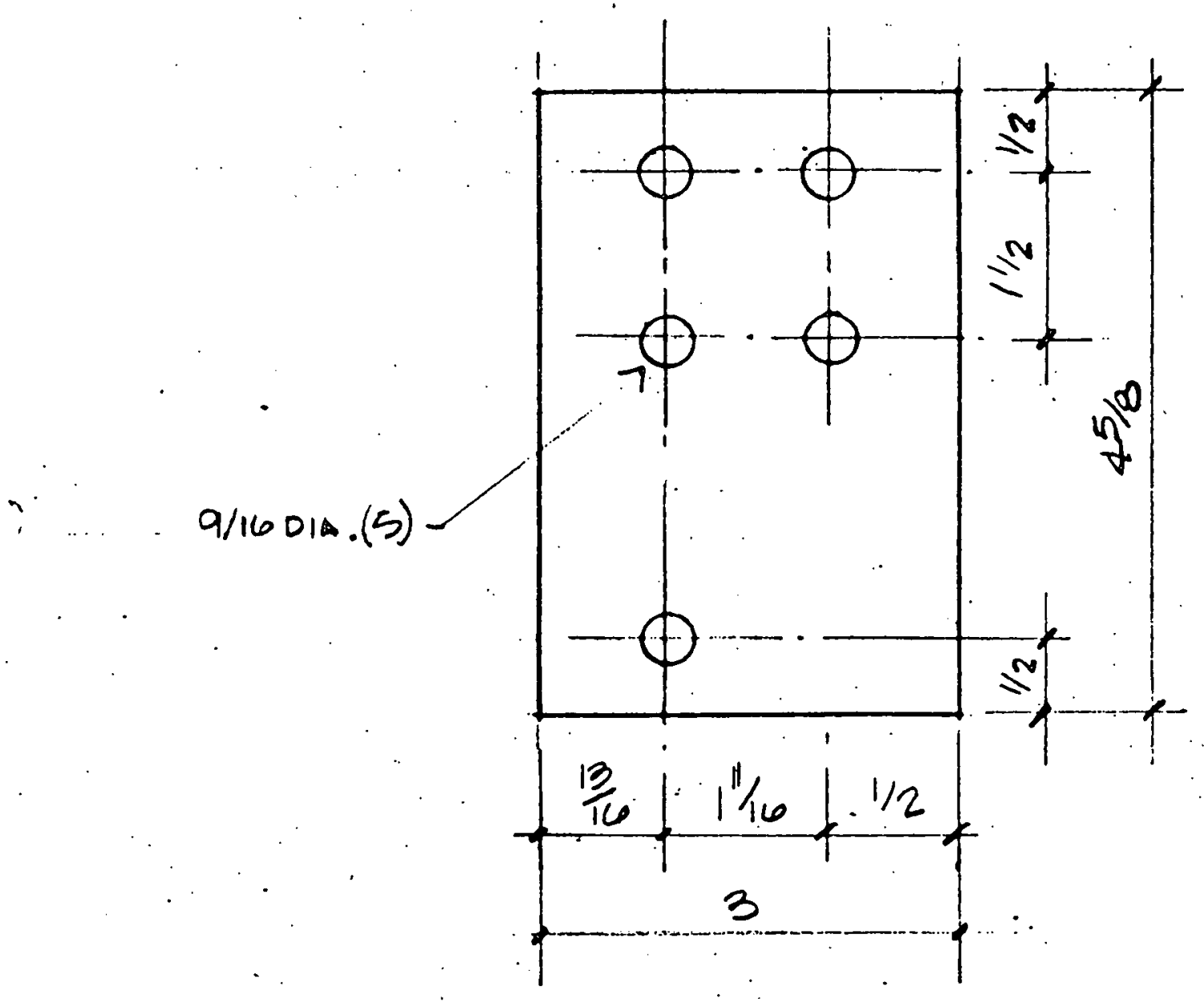

MATERIAL

16 GA. GALV STL $458 \times 3(.096)$

$\ddot{d}$.

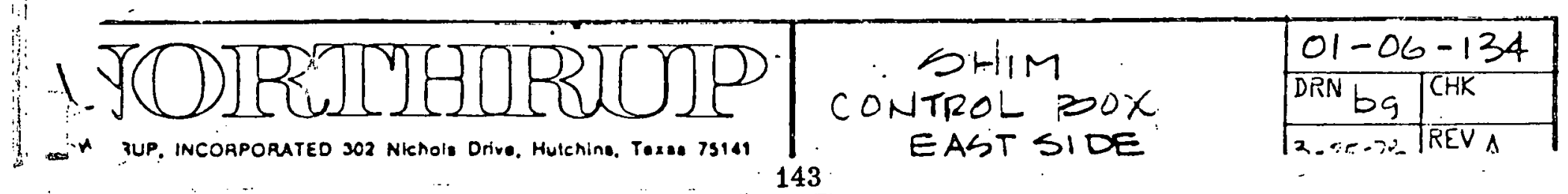




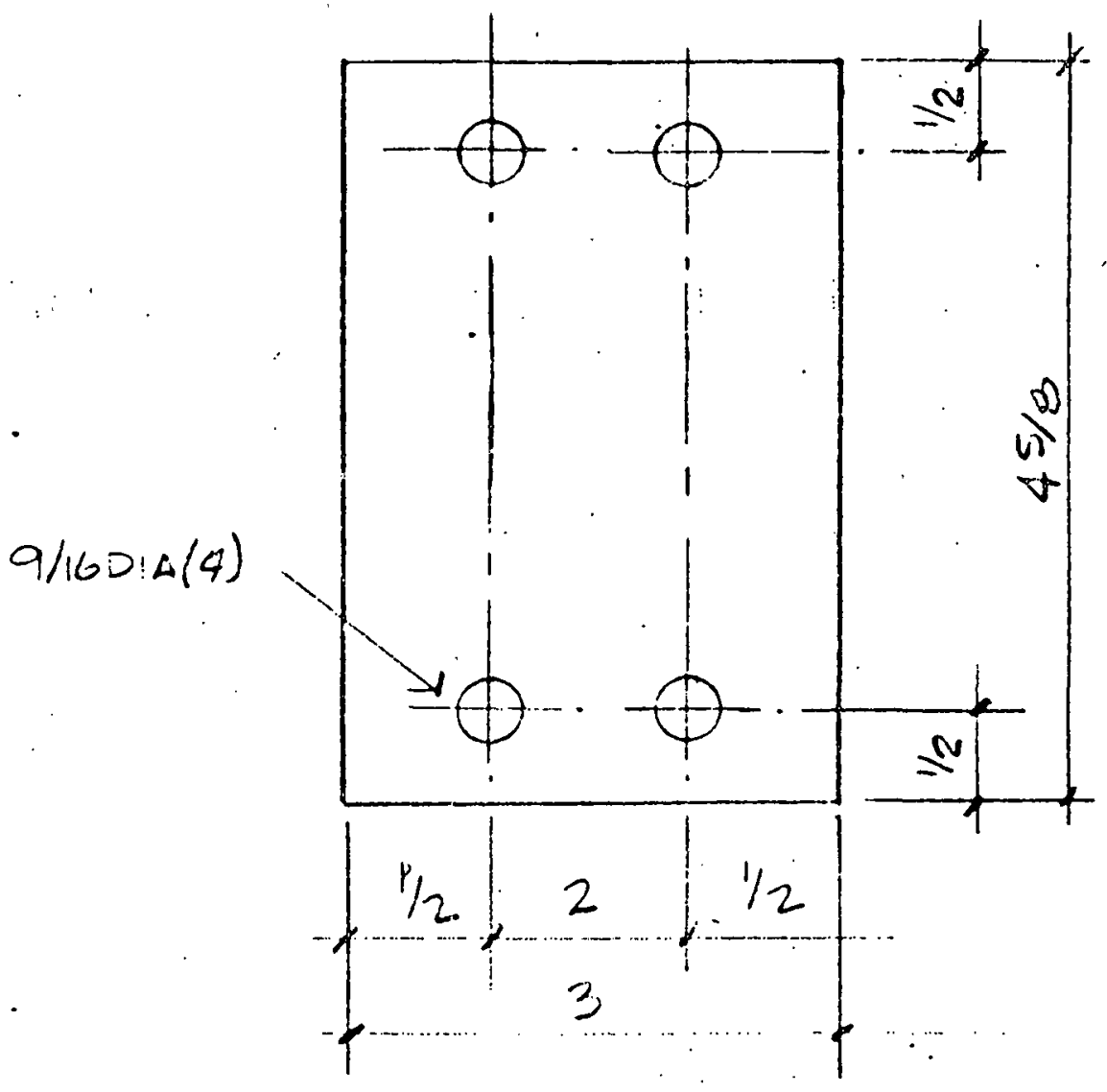

\section{MATERIAL \\ 16 GA. GALV. SR $45 / 2 \times 3(.096)$}

i. 


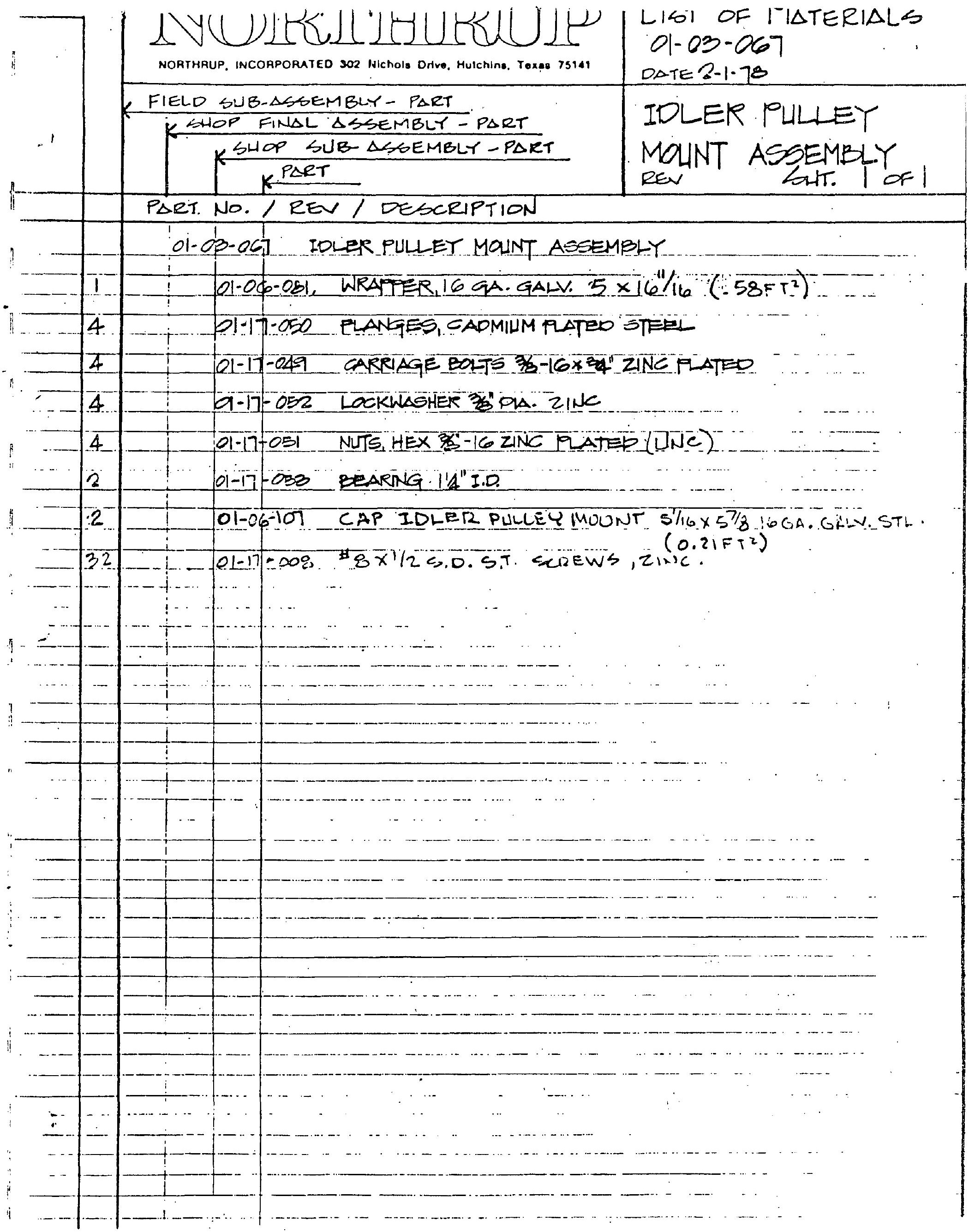

145 


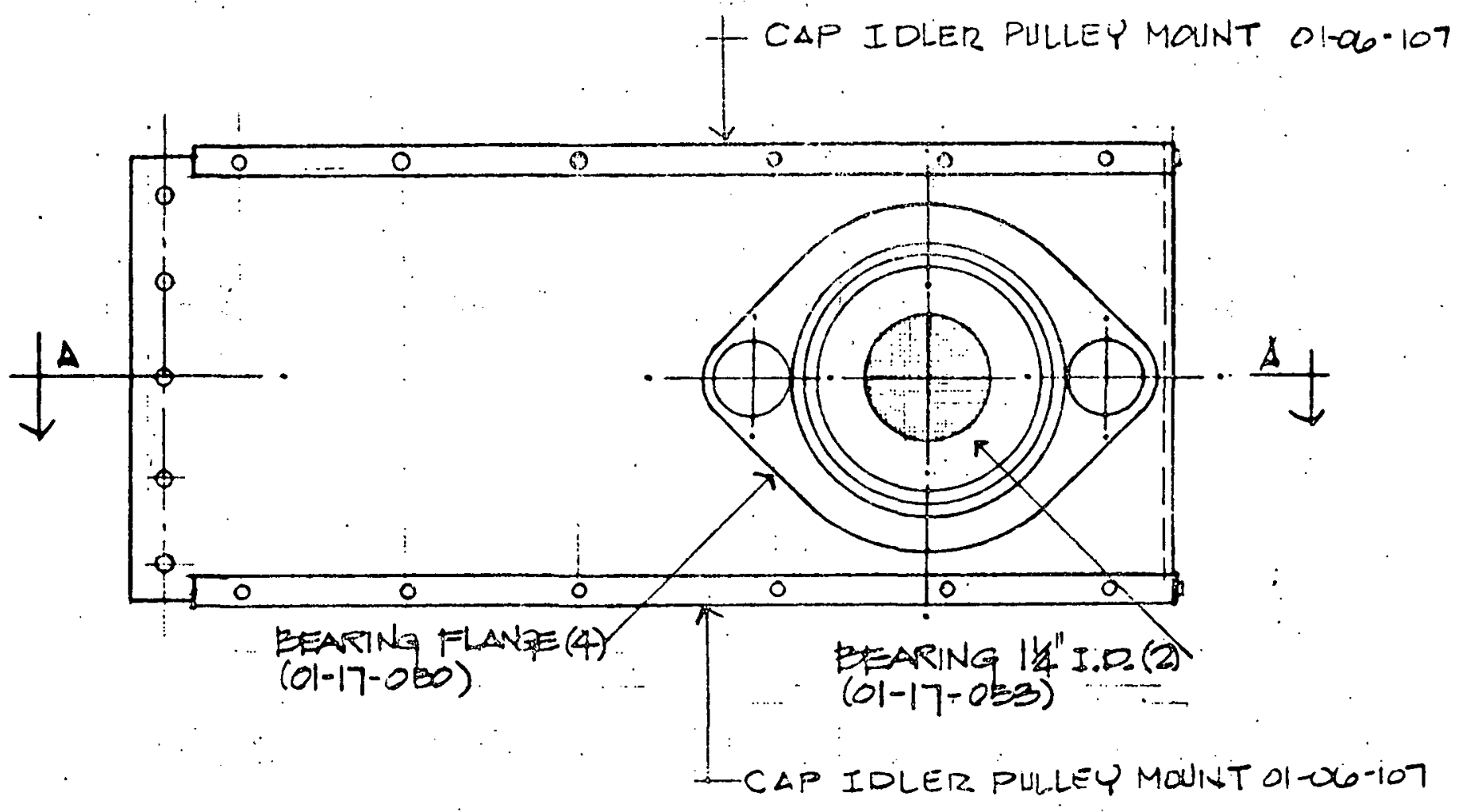

TUP. BADE
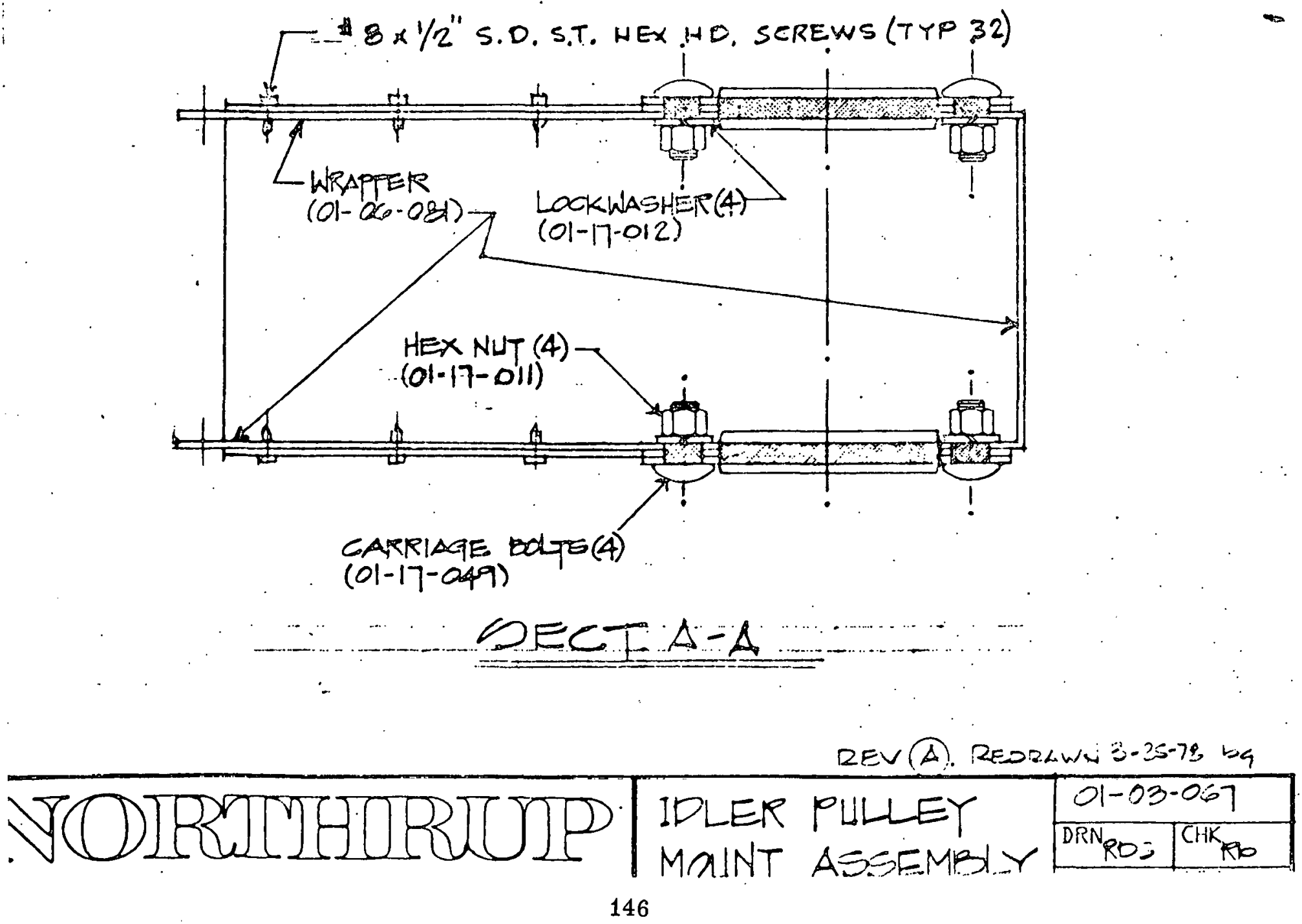


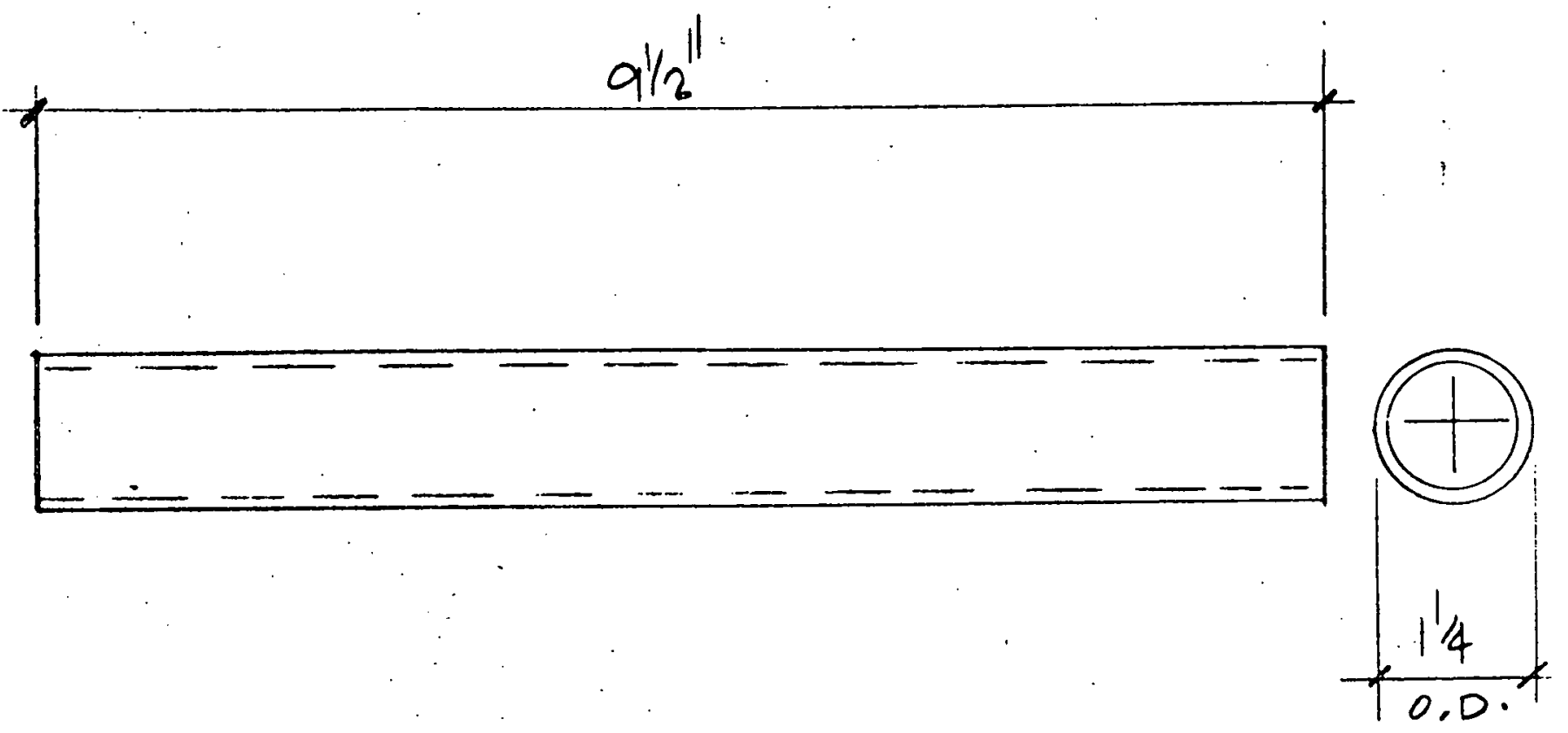

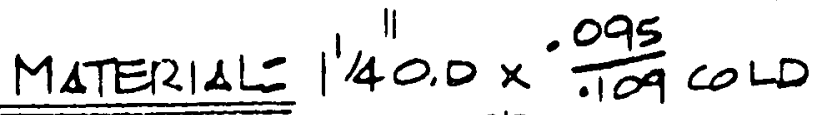

$$
\begin{aligned}
& \text { DRAWH BEAMLESYY TUBIHCA SPEC } \\
& \text { A-S19 MECH. GRADE. }
\end{aligned}
$$

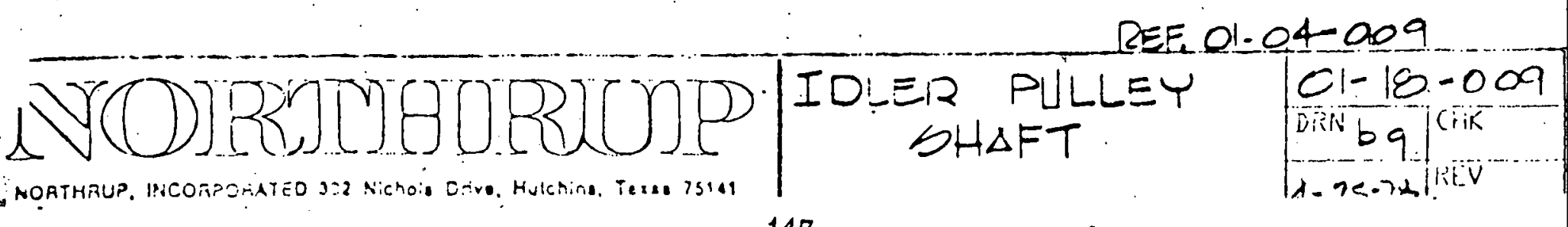


$1+i$
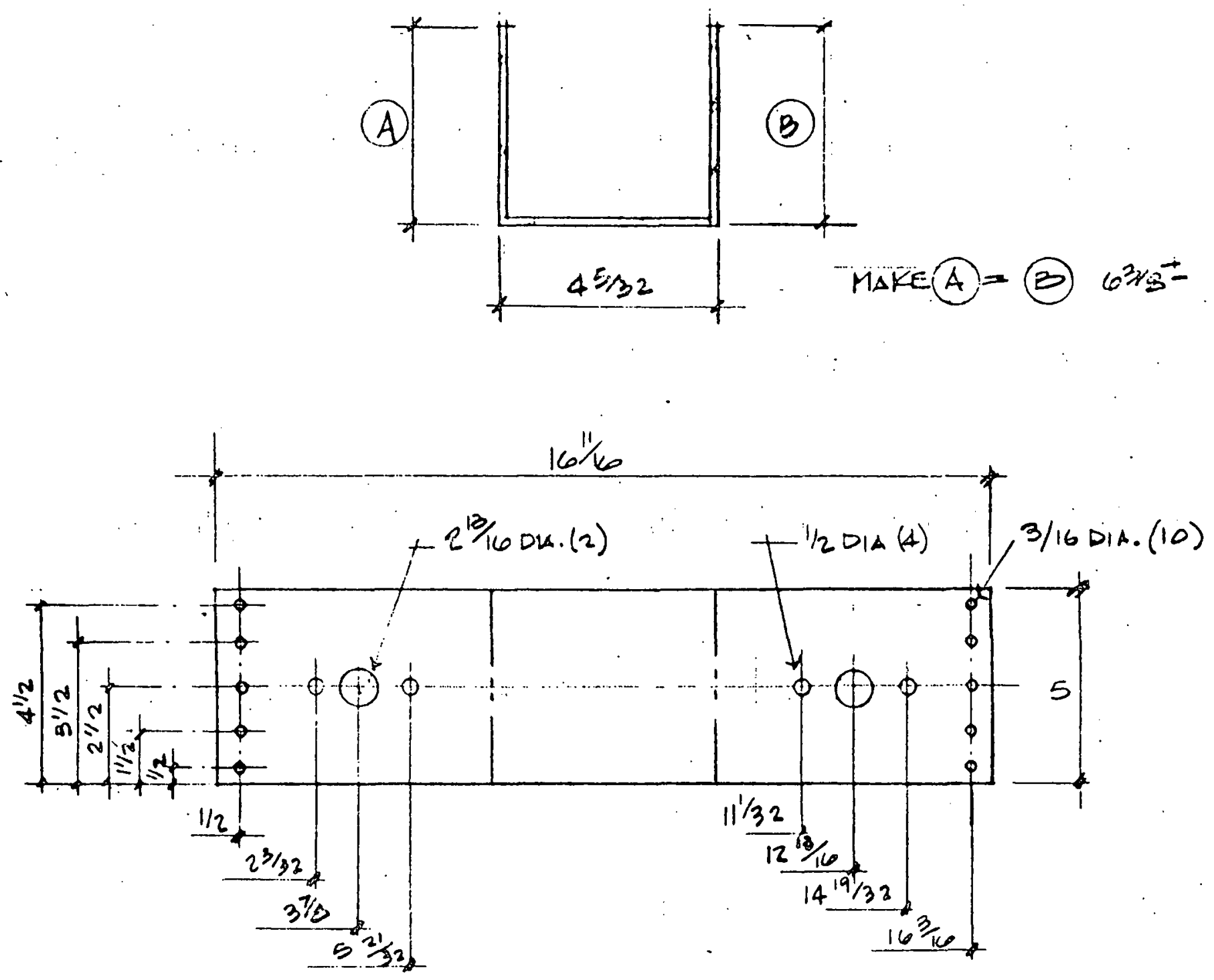

MATERISL:

16 GA. GALV: STL

SHEAR 212E $5 \times 16 \%$ (.58 FT'

d.

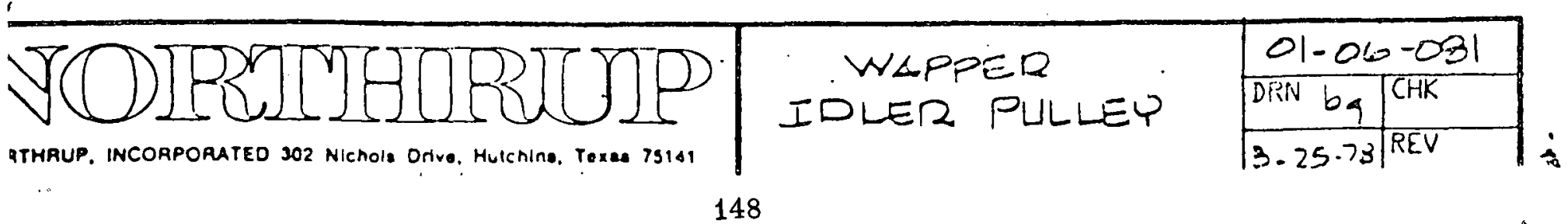


ASPFI?

set Scrov Type.

- Normal Duty
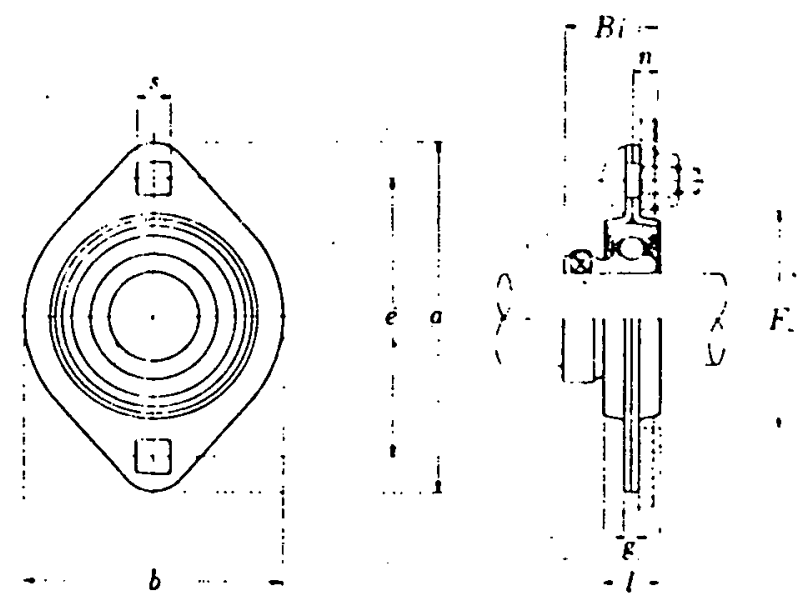

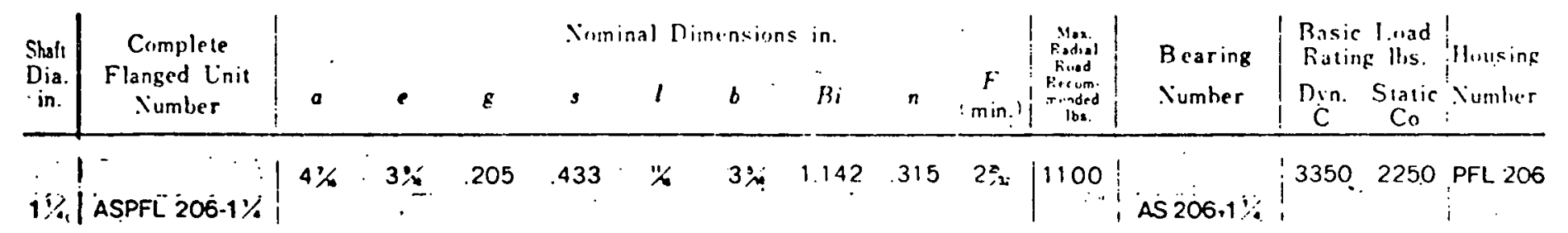

\section{Flanged Units Prossed sicel llousing (Two Bolt)}

\section{Set Screw}

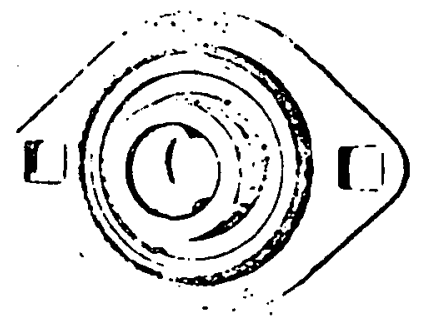

ASPFL 2 (for light load)

With two mounting bolt holes, this like its cast iron counter-part is designed for the least possible mounting space. The bearing housing being separable, this type of unit can be mounted very easily.

HOTE:

FlANGES TO DE ZIHC GOATED

$$
\begin{aligned}
& \text { IDLER PILLEY } \\
& \text { MOLNT BELRIVG }
\end{aligned}
$$

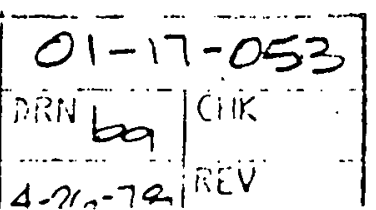



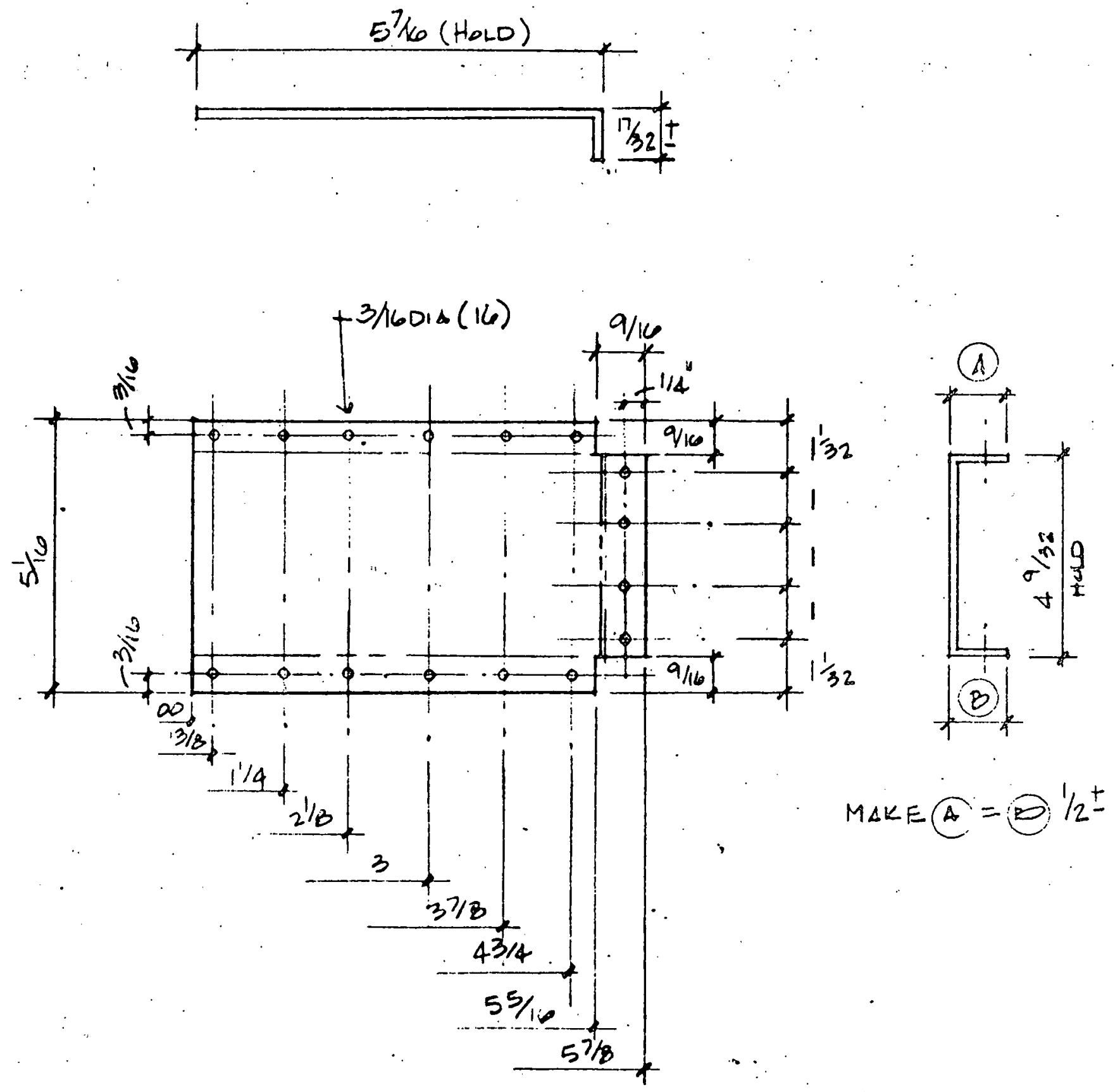

Material:

I6 GA GALV. BTL (0:-001)

$51 / 16 \times 57 / 8 \AA .\left(0.21 \mathrm{Fi}^{2}\right)$

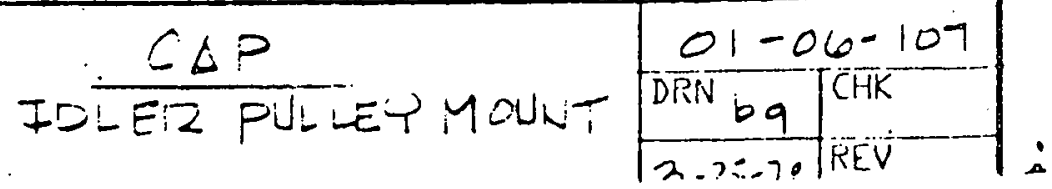




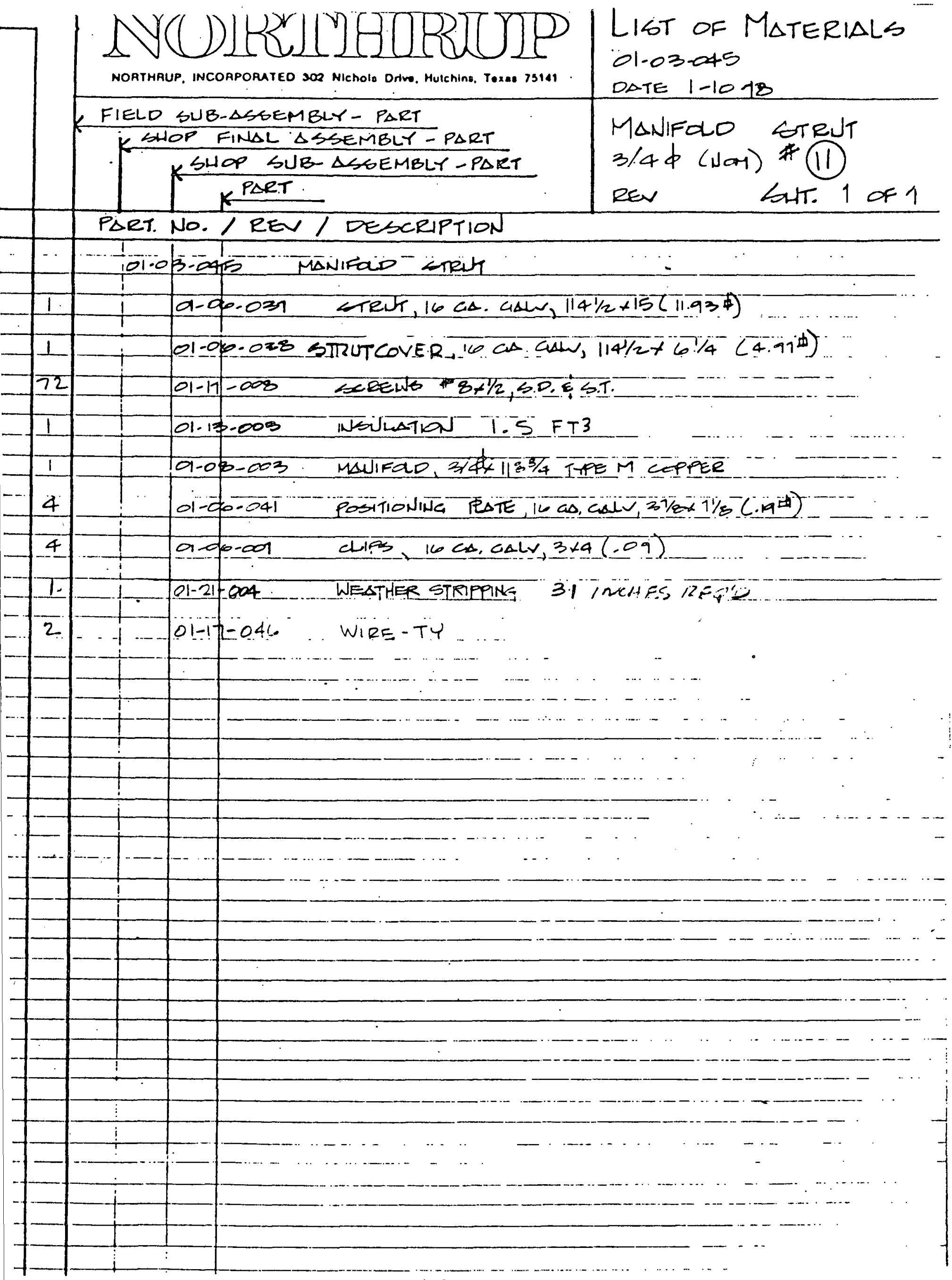




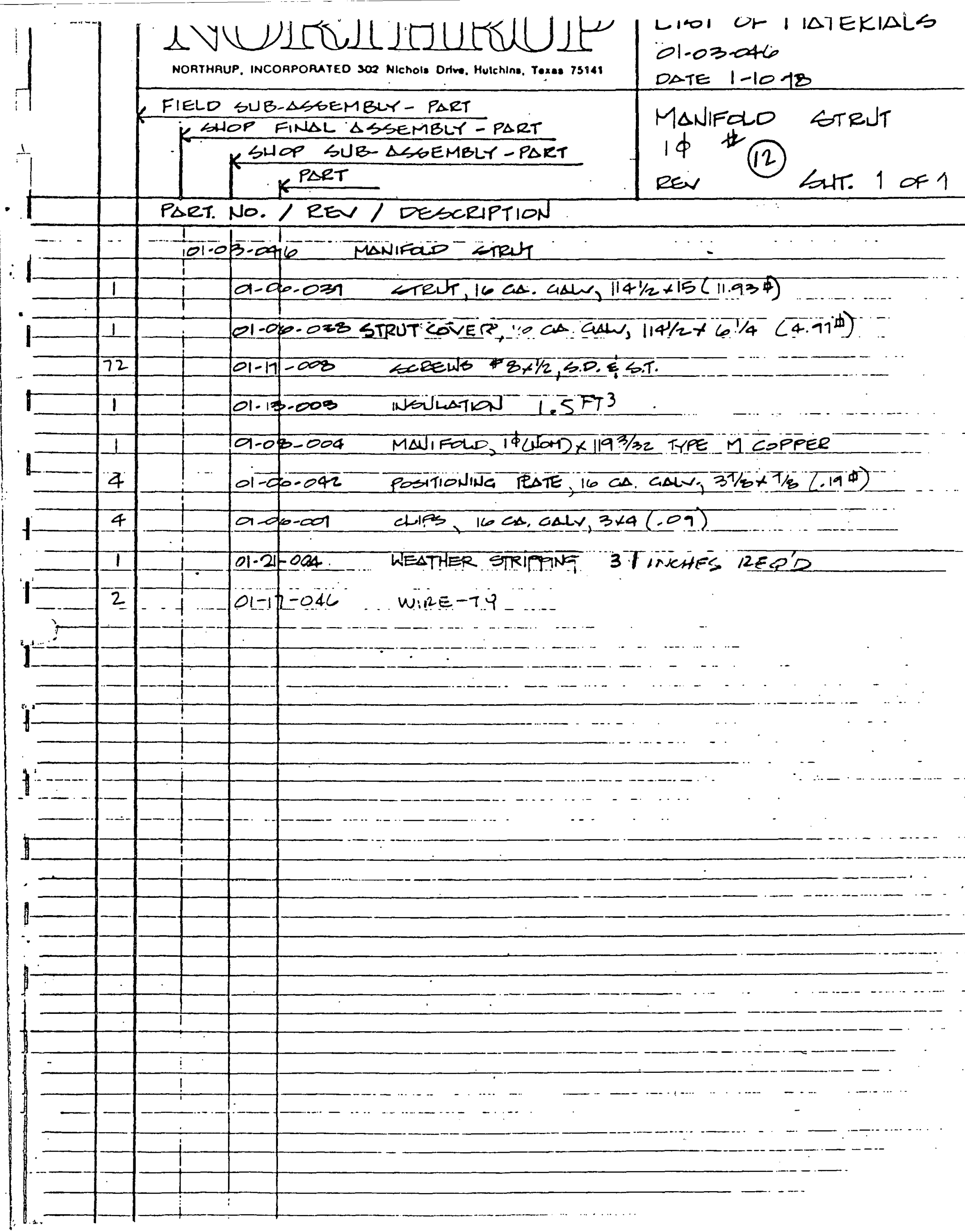




\begin{tabular}{|c|c|}
\hline AY(l) & $\begin{array}{l}\text { LIST OF MATERIALS } \\
\text { OI-O3.04T } \\
\text { DATE } 1-10.18\end{array}$ \\
\hline 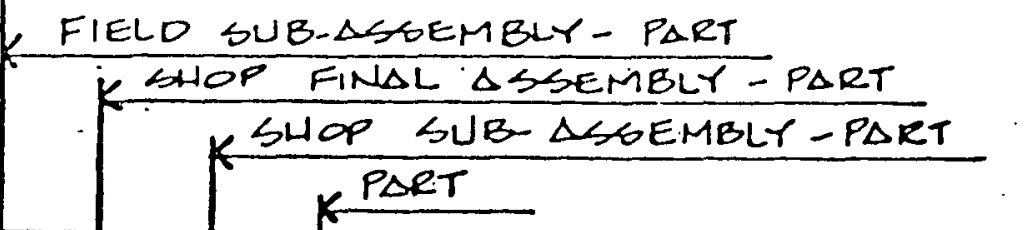 & 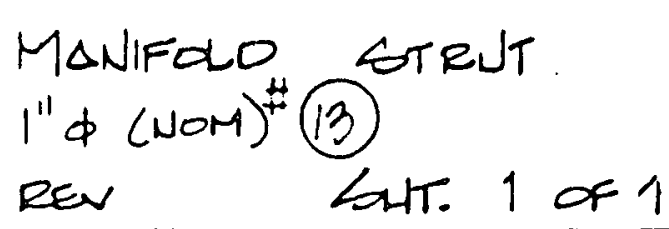 \\
\hline
\end{tabular}

PART. NO. / REV / DESCRIPTION

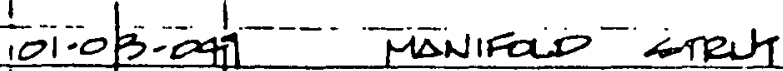

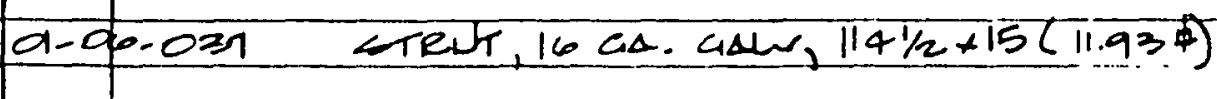

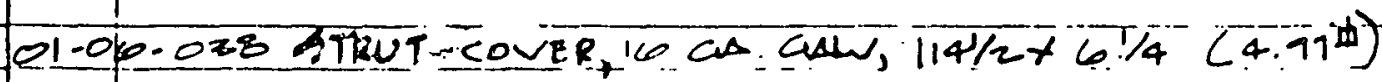

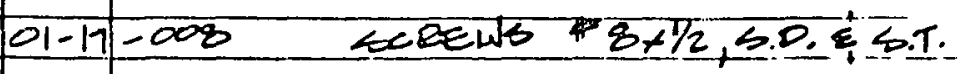

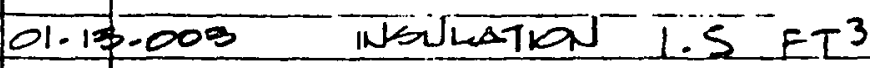

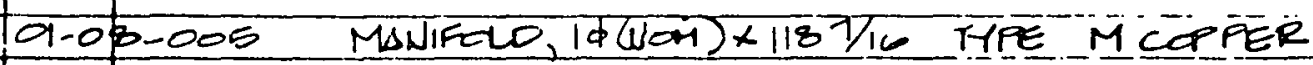

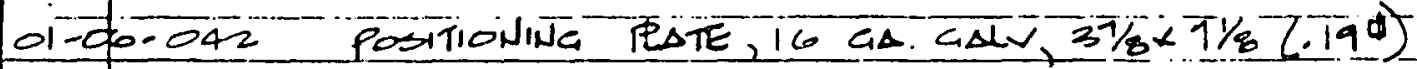
aloo-or chies, $16 \mathrm{cs}, 04 \mathrm{lv}, 3 \times 4(.09)$ 9-2H-Q4 ... WEATHER STRIPPINA. ST INCHES REQ'O 


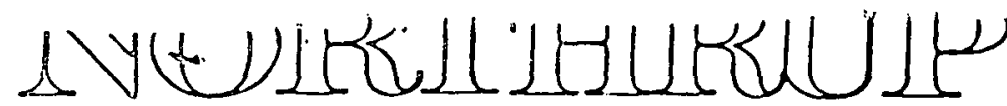

NONTHAUP. INCOAPORATEO 302 Nichole Drive. Hulchins, Texạt 75141

FIELD SIUB-DGEEMBLY - PART

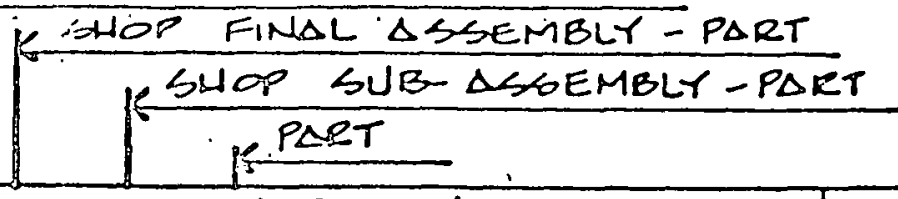

PART. NO./REV/ DESCRPTION

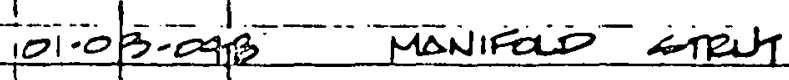

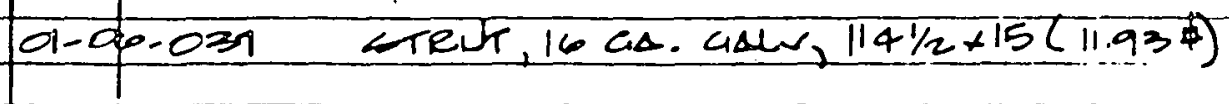

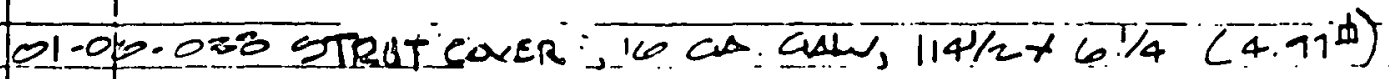

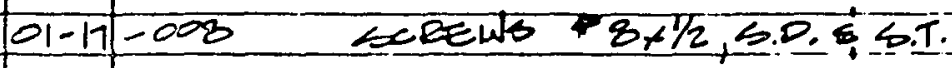

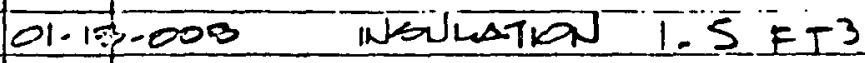

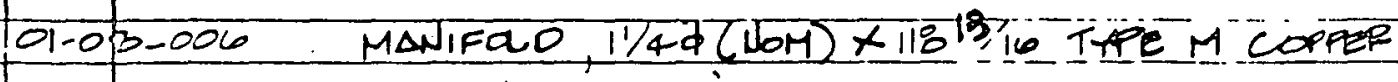

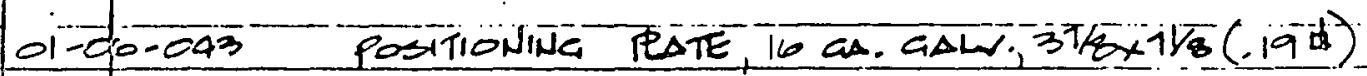

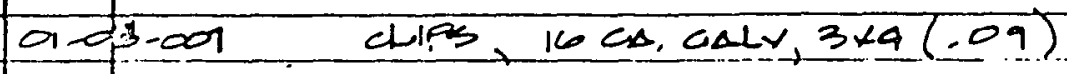

$01.21,024$. WEATHER STITPNG 31 INCHES REQTO

$01-17=046 \ldots \omega_{12}-t+q$ 

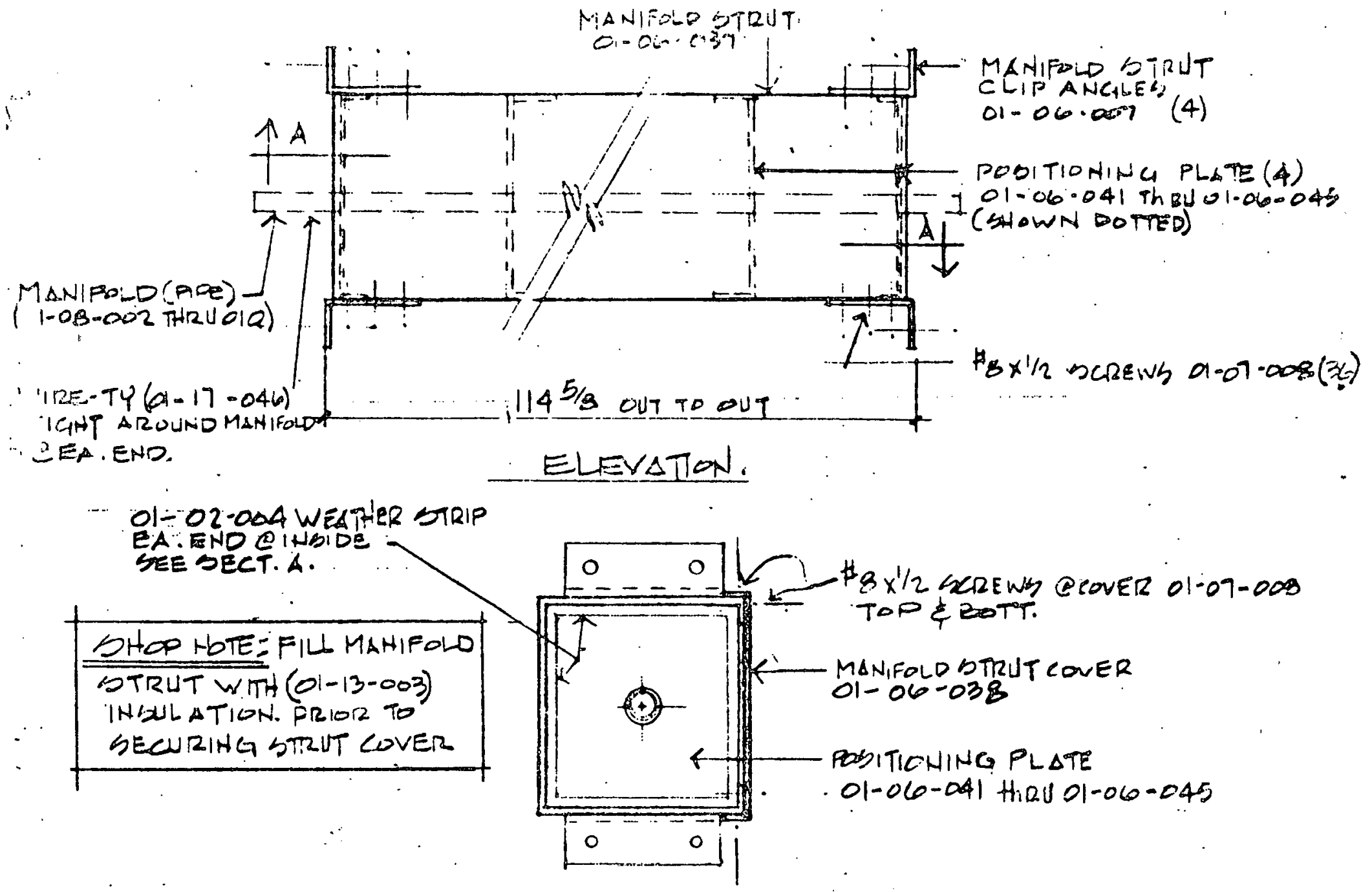

$+$

$\because$

$\because$ NOTE:

OCATIONOF FAETENERG VETERMINED BY PILOT "roteE' IN SOTUIT ( FOR OSITIONING PE'SAND ITRU T CONER:)

\section{TYP ENOVIEW}

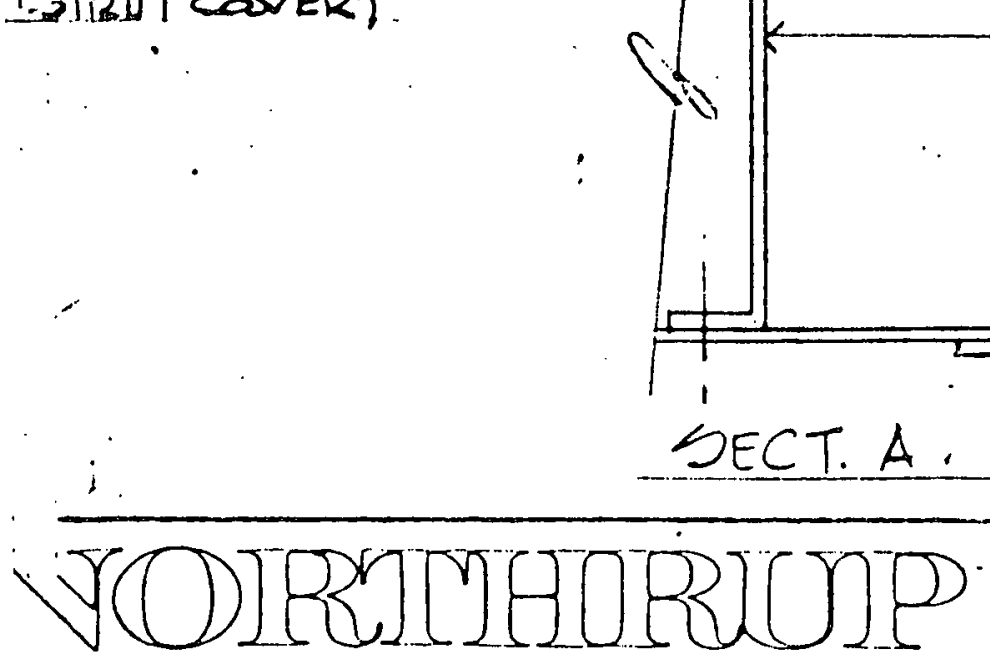

IMRUP. INCOAPORATED 302 NIChole ORIVe. Hulchine. TOIas 75141

O-O6-OOT MANIFOLS STIZUT CLIP ANCLLE

POSITININC PL DTE $01-06-041$ MHIJ $d-06-045$

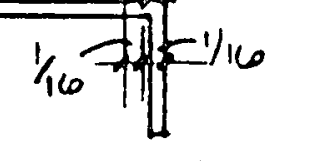

-O2 -OD4 WE ATHER.ETRIP. i. OETALLE NOT TO SALE b) REY 3.27.7\& 2EDR

MAN:FOL STRUT

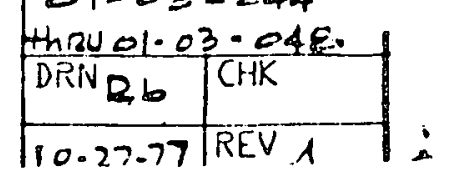



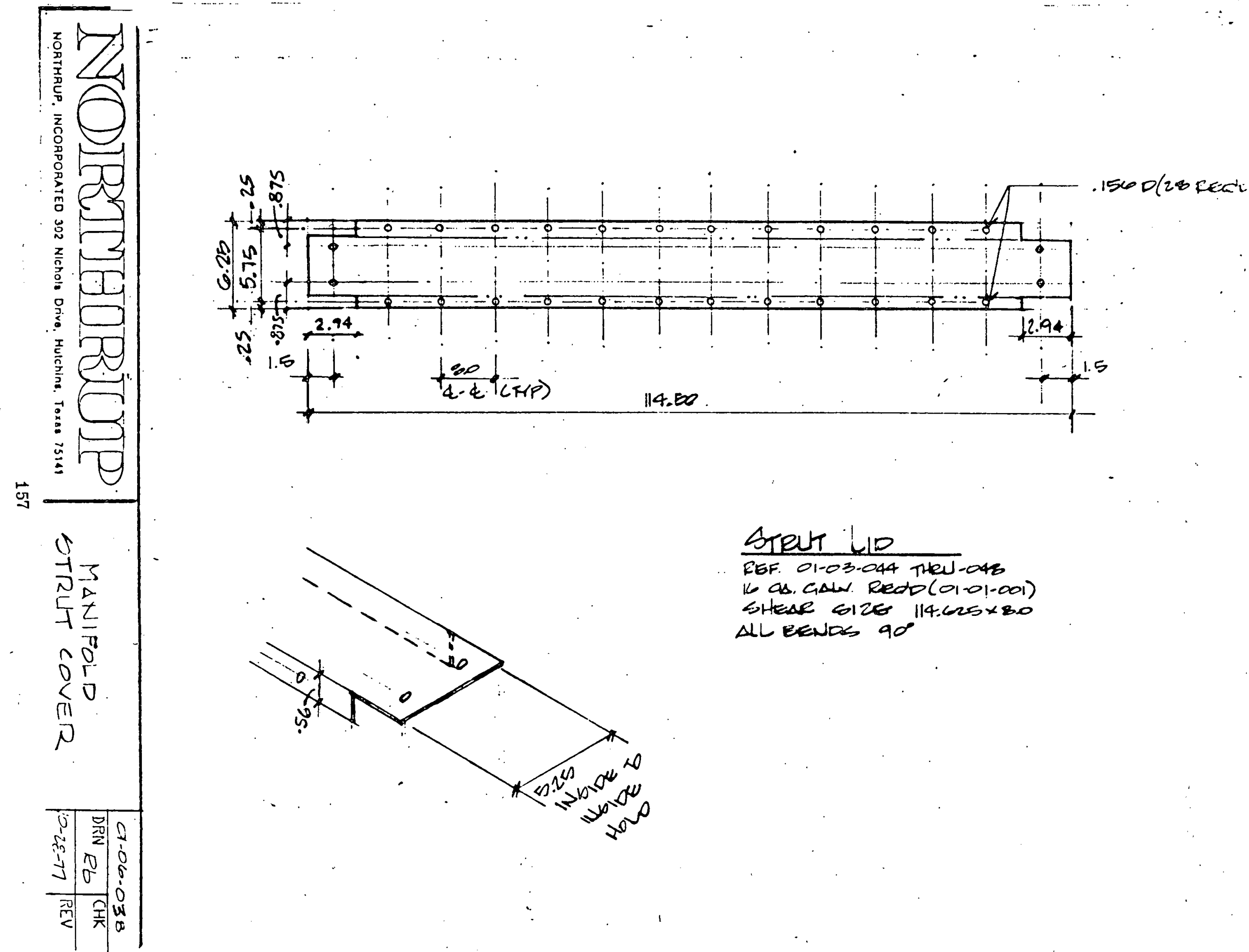

\section{STRUT UD}

EEF. O1-03.044 THEU-045

$K$ as. Gavw. Redp $(0101-\infty 01)$ SHeare Giver $114.025 \times 30$ ALL ENENAS $90^{\circ}$ 

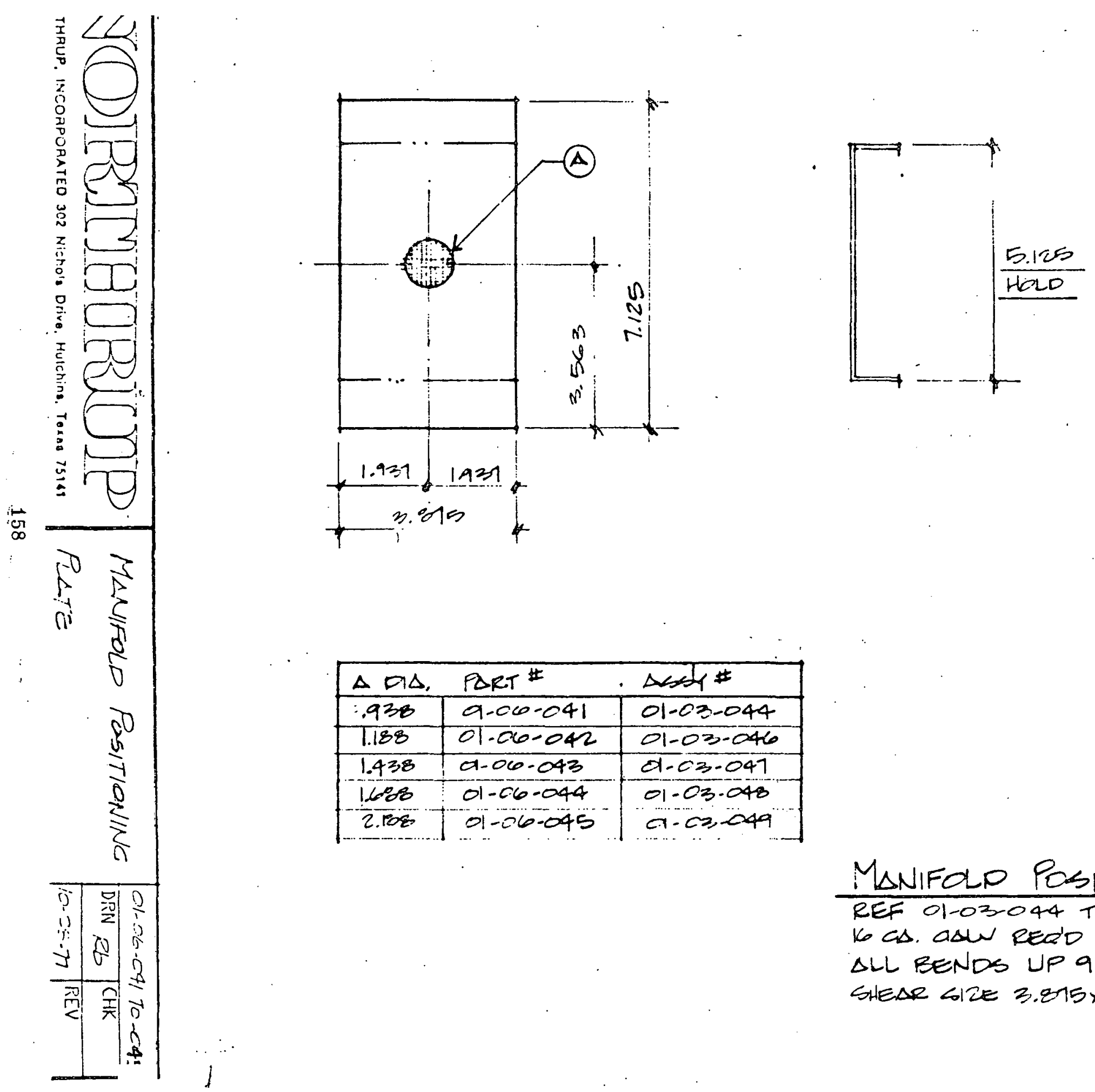

\begin{tabular}{|c|c|c|}
\hline$\triangle M \Delta$, & PQRT & $\Delta$ cs $\#$ \\
\hline$: .938$ & $9-06-041$ & $01-03-044$ \\
\hline$T .188$ & $01.06-042$ & $01-03-046$ \\
\hline 1.438 & $0.06-043$ & $0-c 3-047$ \\
\hline 1688 & $01-0.6-044$ & 01.03 .048 \\
\hline 2.106 & $01-06-045$ & $a-c \geq-\infty 49$ \\
\hline
\end{tabular}

Manifolp Positionina plate REF $01-03,044$ THRU -040 16 aS. ASW REA'D (O1-01-001) $\triangle L L$ BENDS UP $90^{\circ}$

Shear cize $3.875 \times 7.125$ 


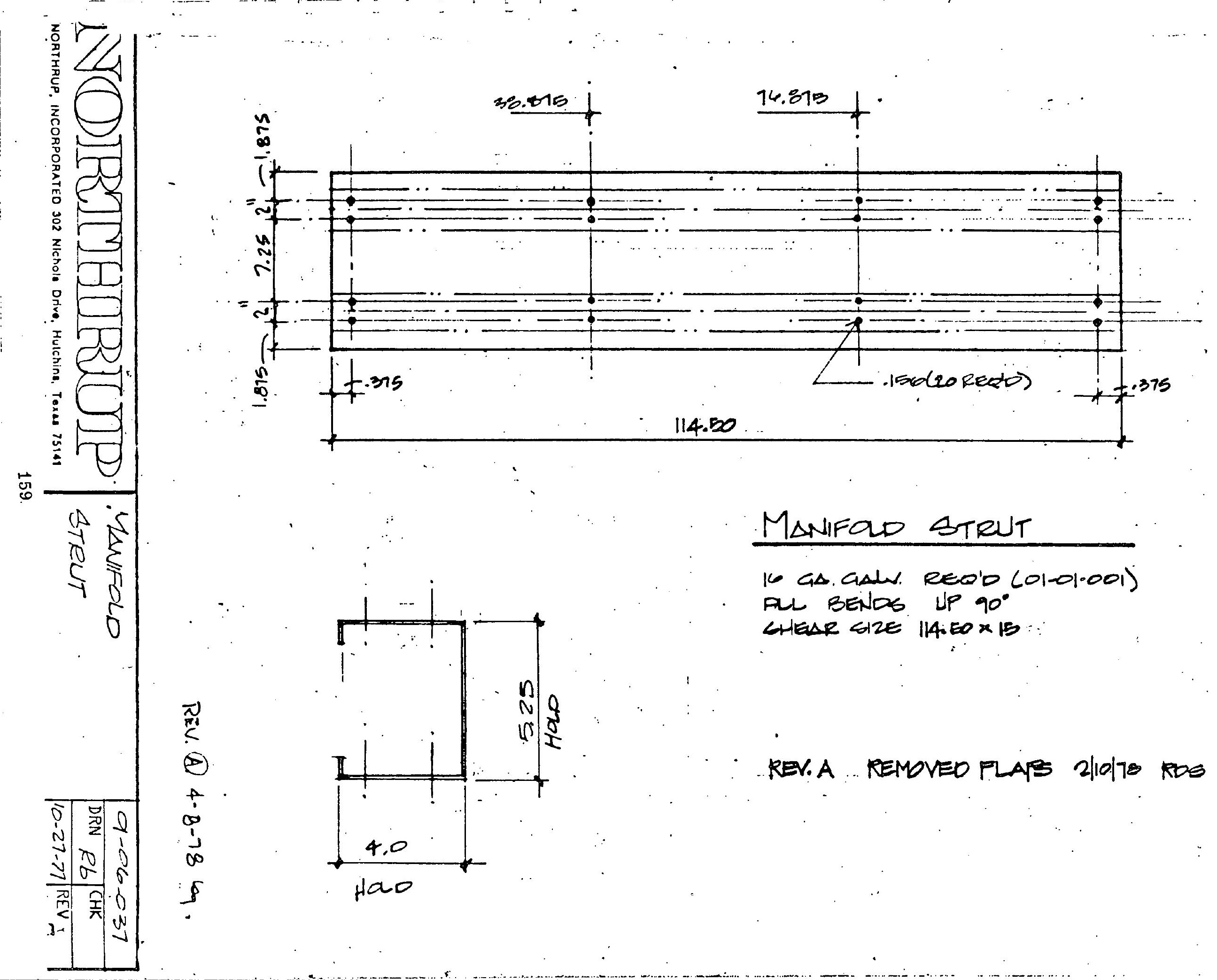



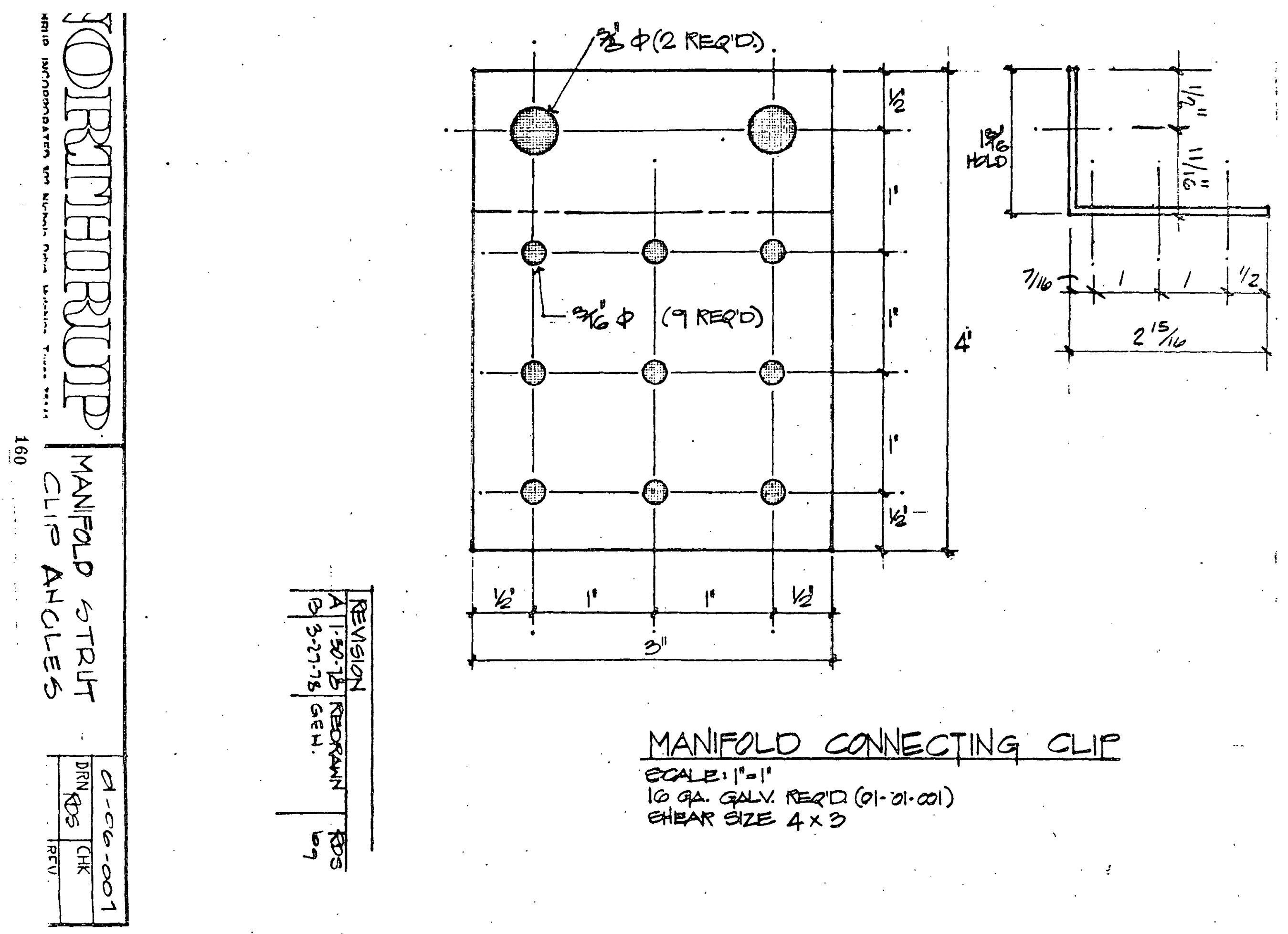


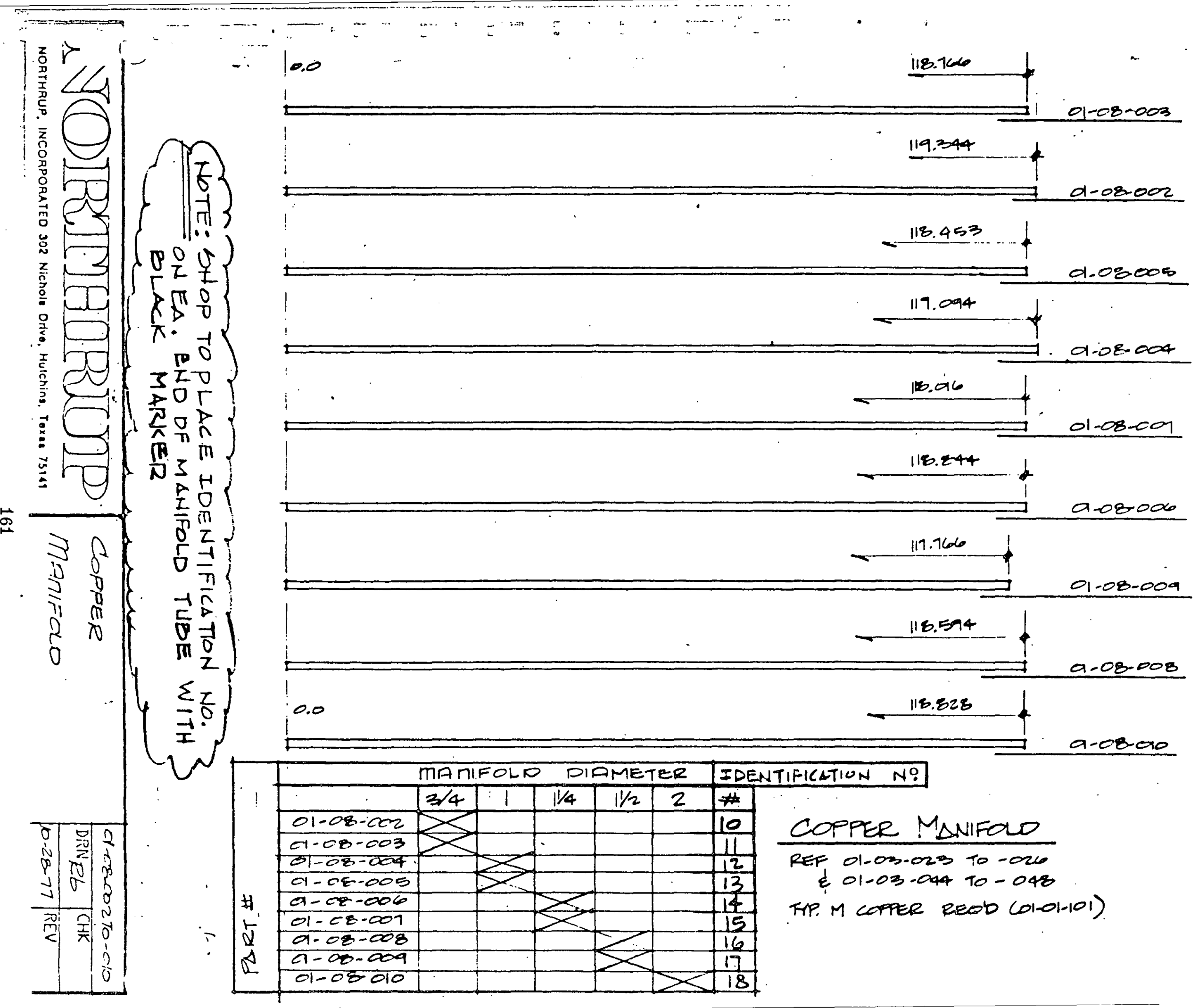




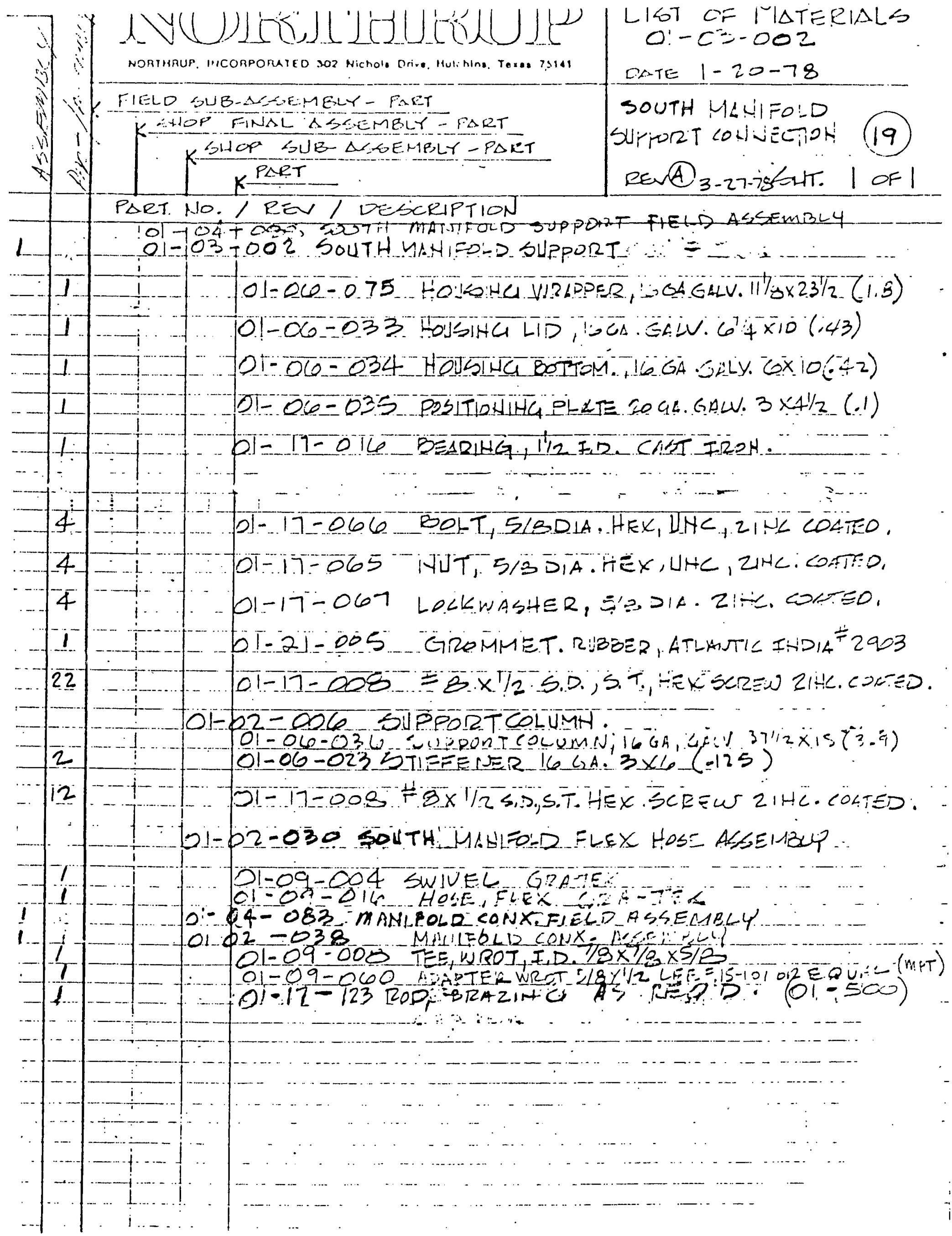




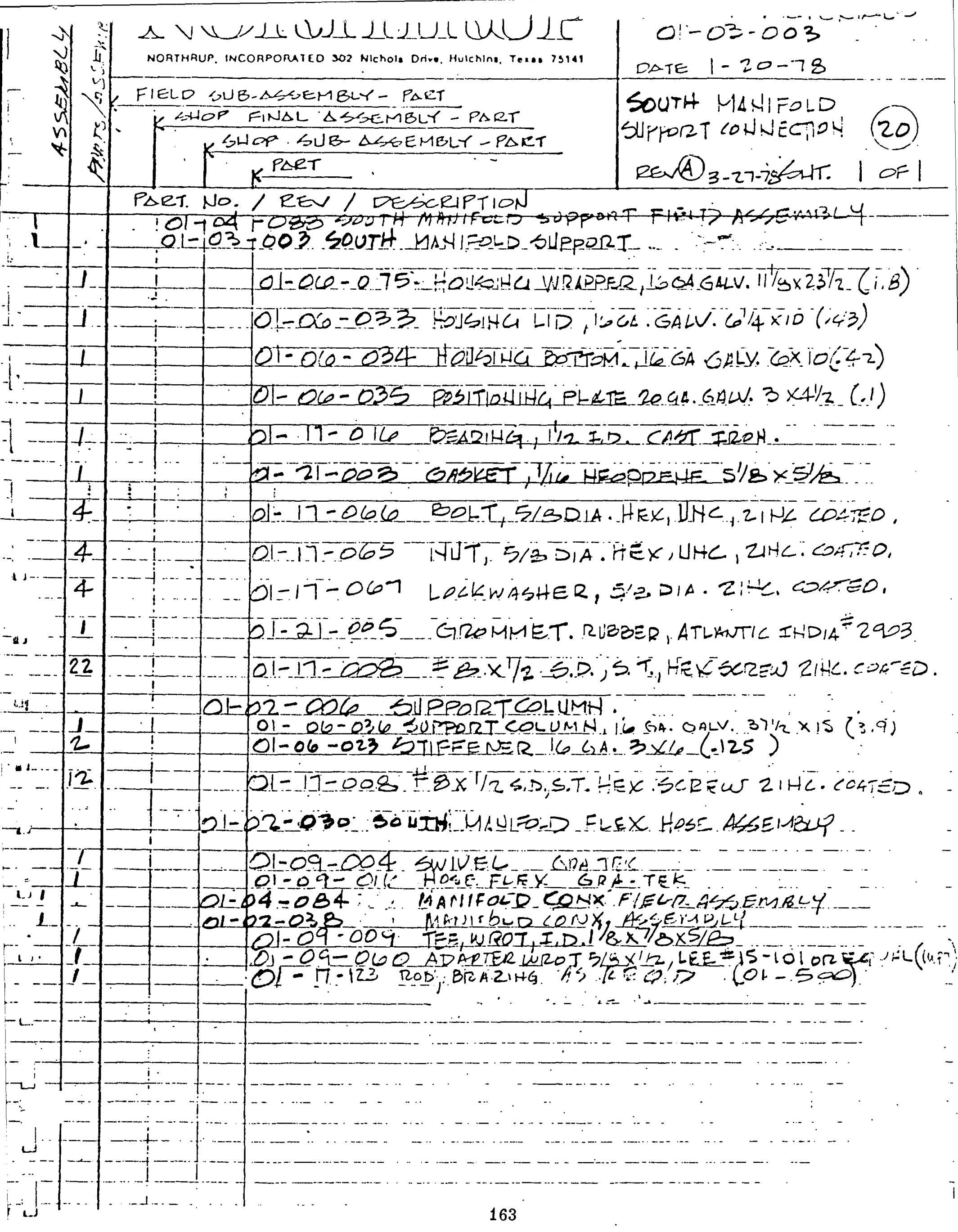




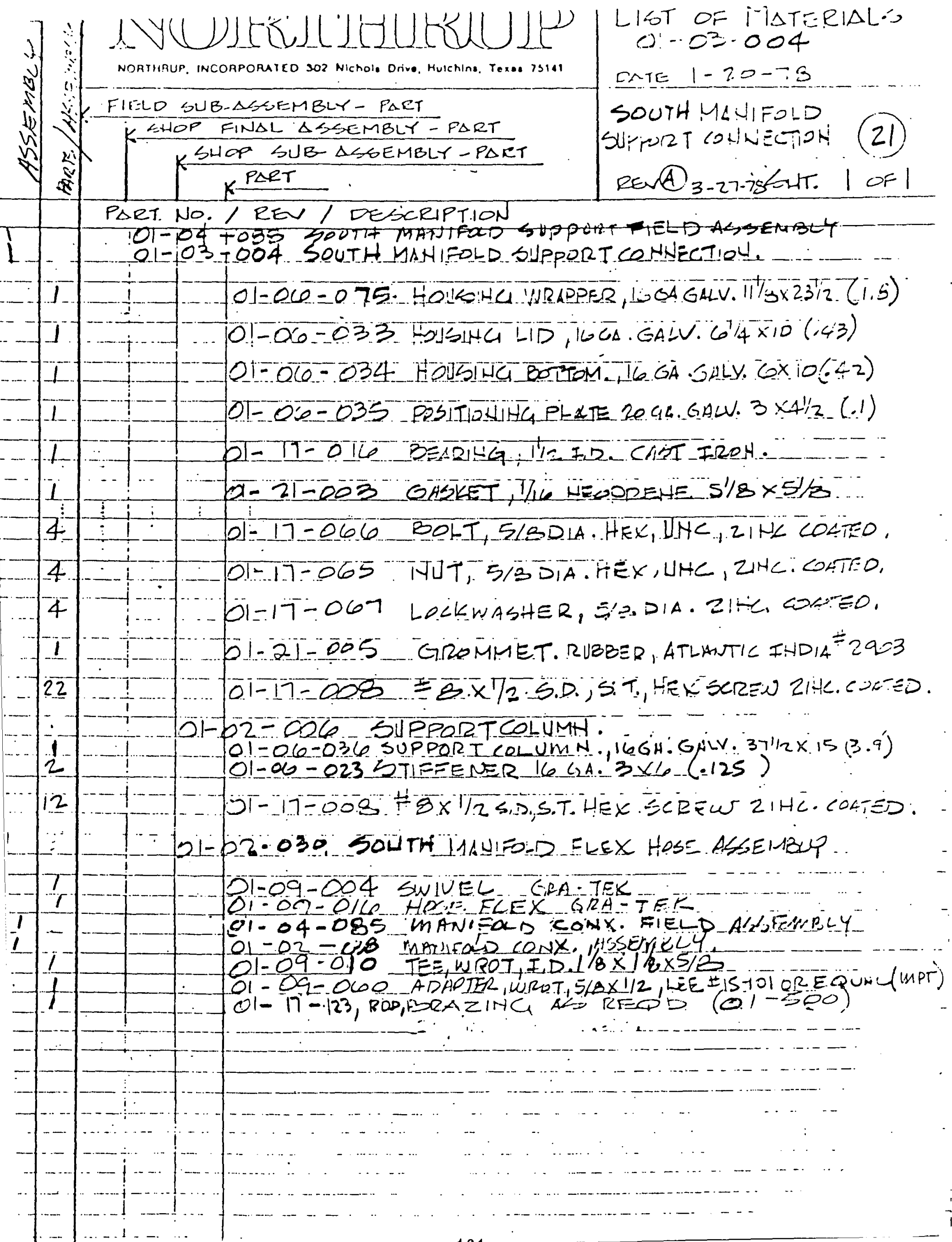




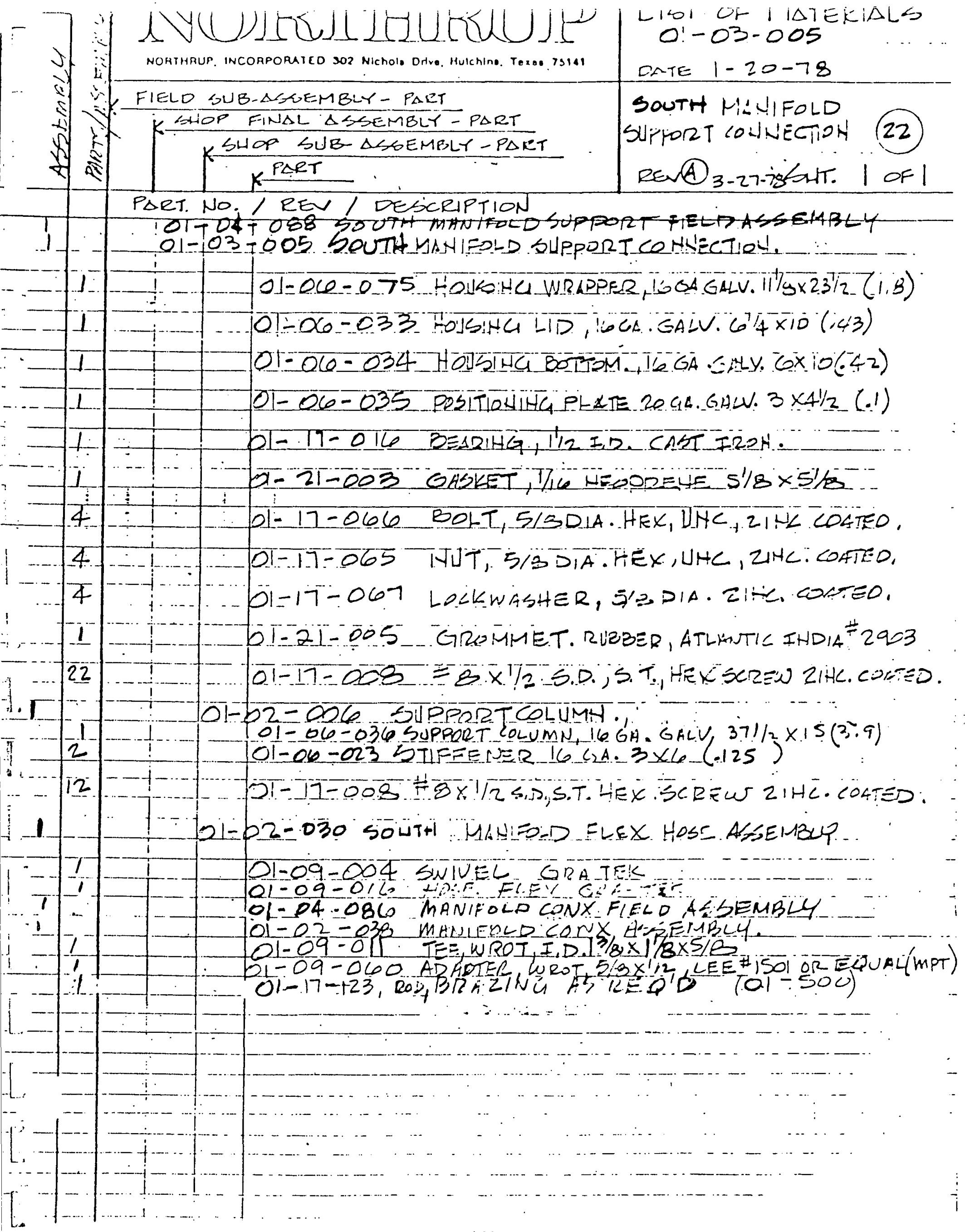




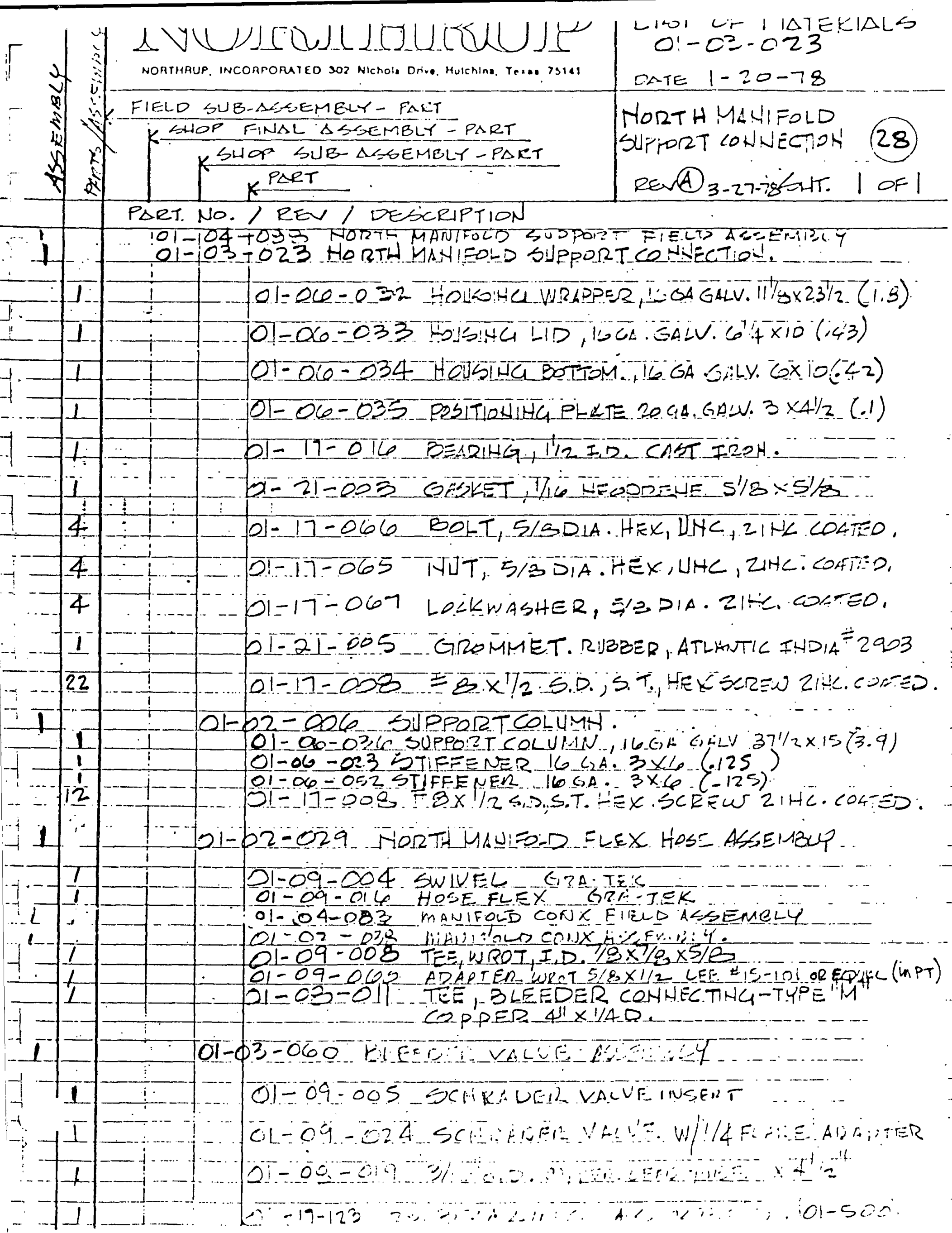




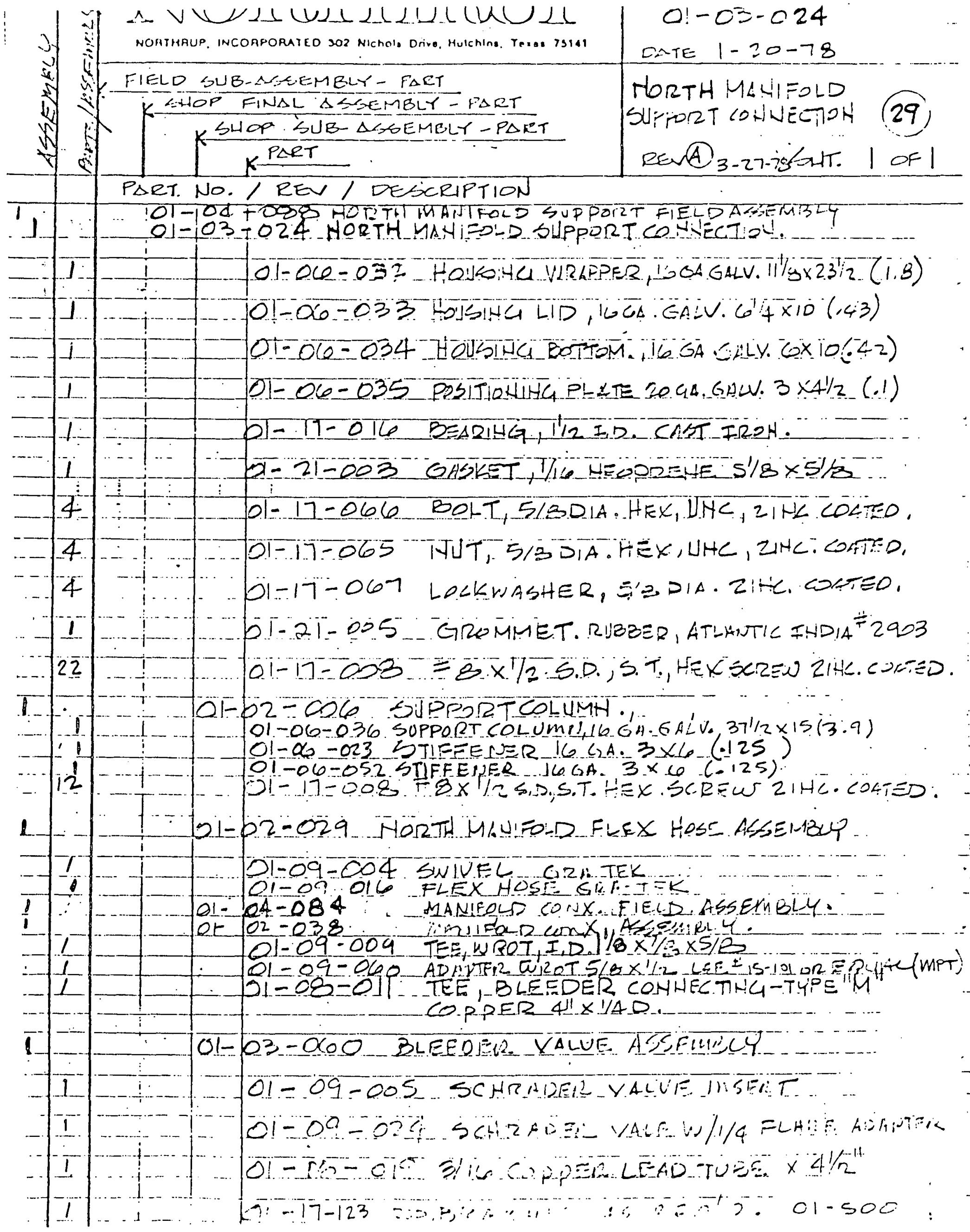




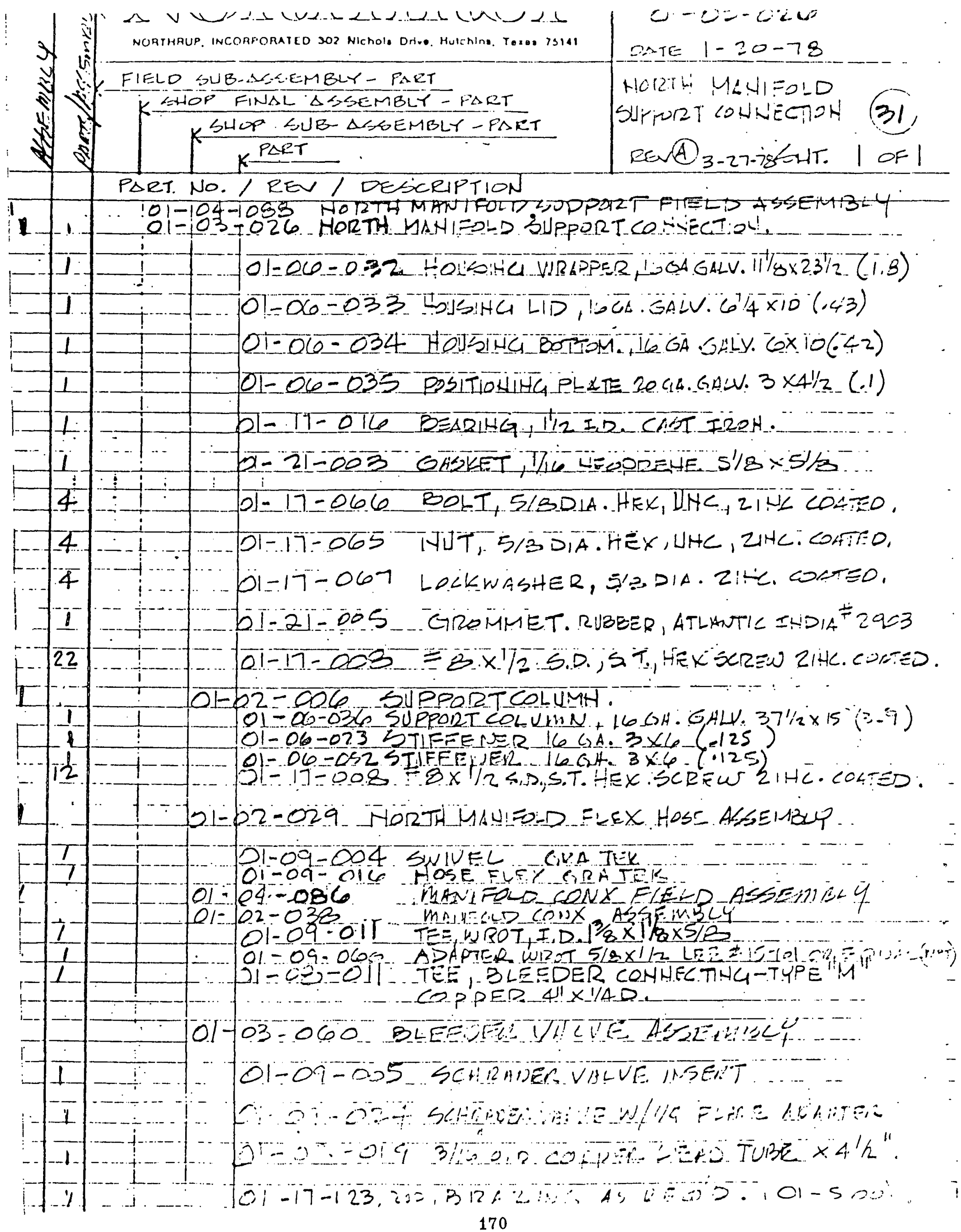




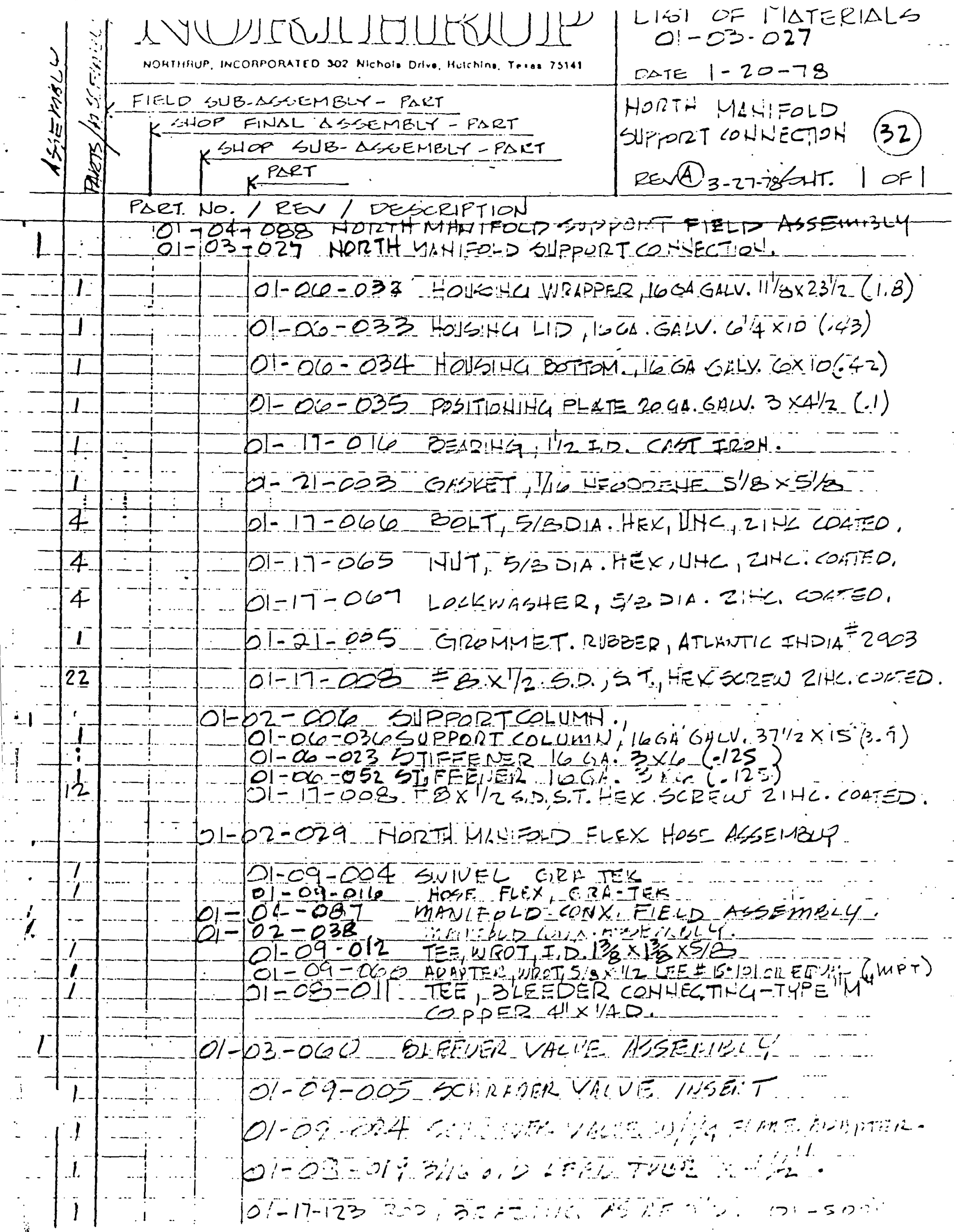




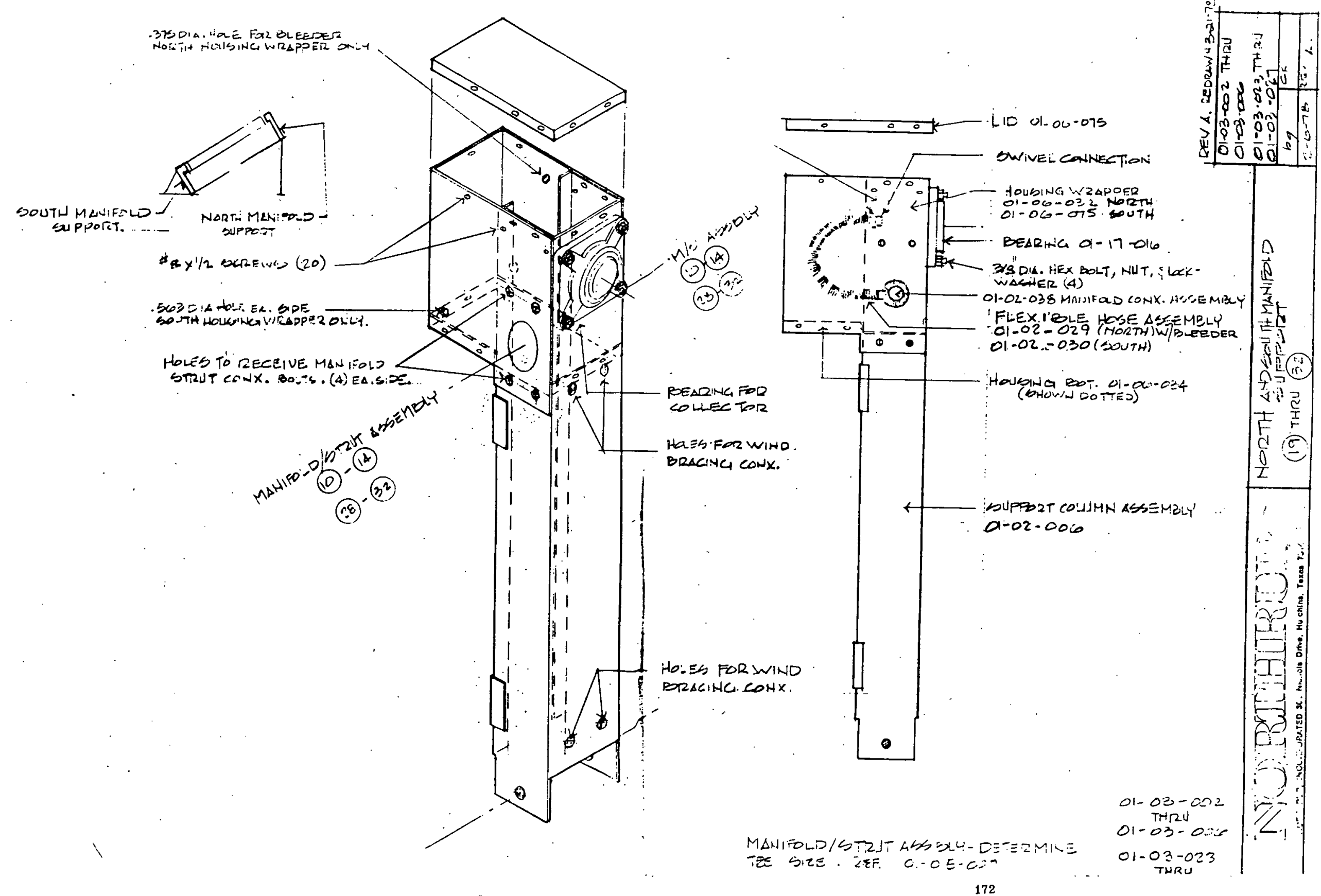




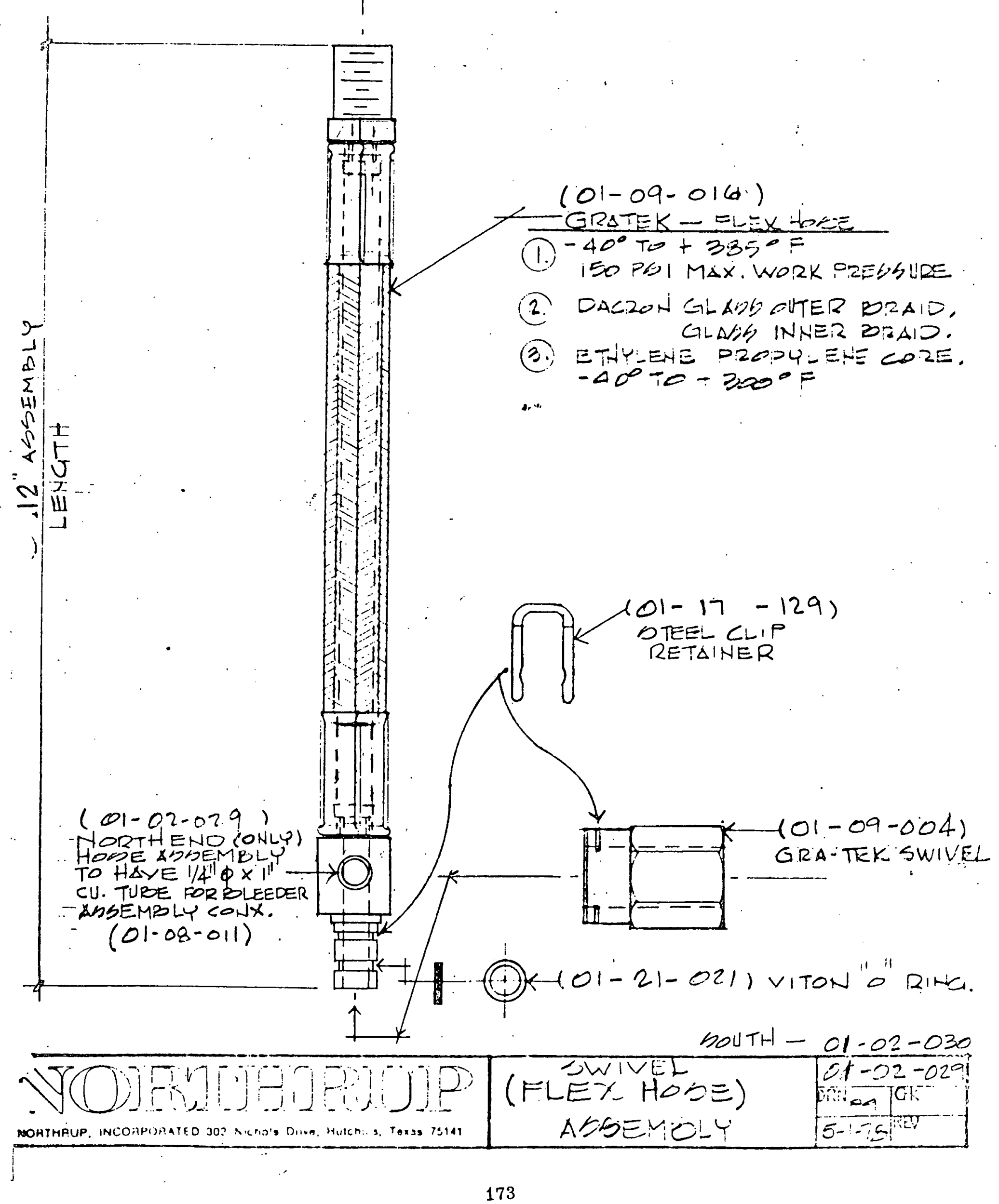




\begin{tabular}{|c|c|c|c|c|}
\hline 214 & TEE PADI H & LUH MSFR & $i=E=3 ! 25$ & GDADTER PADT UU \\
\hline 2 & $\begin{array}{l}01-09-008 \\
01-09-009\end{array}$ & $\begin{array}{l}19 \\
70\end{array}$ & $\begin{array}{l}3 / 4 \times 3 / 4 \\
1 \times \geqslant 14\end{array}$ & $01-09.060$ \\
\hline$i$ & $81=04=009$ & 21 & $\mid \begin{array}{l}x \geqslant 14 \\
x \times 1\end{array}$ & \\
\hline 1 & $01-09-011$ & 22 & $11 / 4 \times 1$ & \\
\hline 2 & $01-09-012$ & 23 & $11 / 4 \times 11 / 4$ & \\
\hline 2 & $01-09-008$ & 28 & $3 / 4 \times 3 / 4$ & \\
\hline 1 & $01-09-09$ & 29 & $1 \times 3 / 4$ & \\
\hline 1 & $01-09-010$ & 30 & $1 \times 1$ & \\
\hline 1. & $01-09-011$ & 31 & $11 / 4 \times 1$ & \\
\hline 2 & $01-09=012$ & 92 & $+1 / 4 \times 1 / 4$ & $01-0,000$ \\
\hline
\end{tabular}
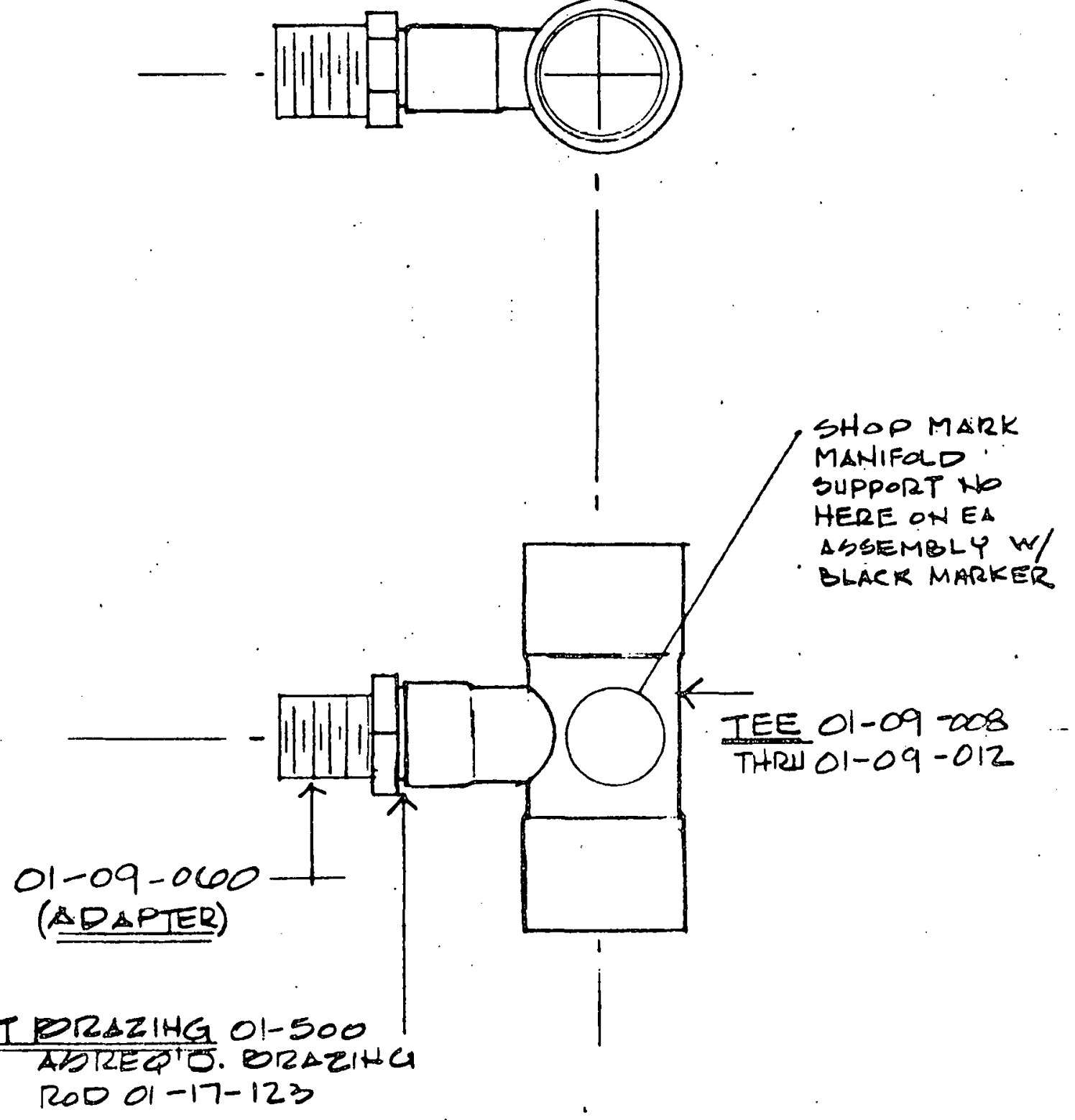

\begin{tabular}{|c|c|c|}
\hline 101011010 & $\begin{array}{c}\text { MANIEAD CONX. } \\
\text { LASEMBLY }\end{array}$ & $\frac{0-02-038}{\text { Crintoq }}$ \\
\hline MAUP. INCORPORAIED 302 NICHOIS & & $5.1-7 \delta \mid R E$ \\
\hline
\end{tabular}



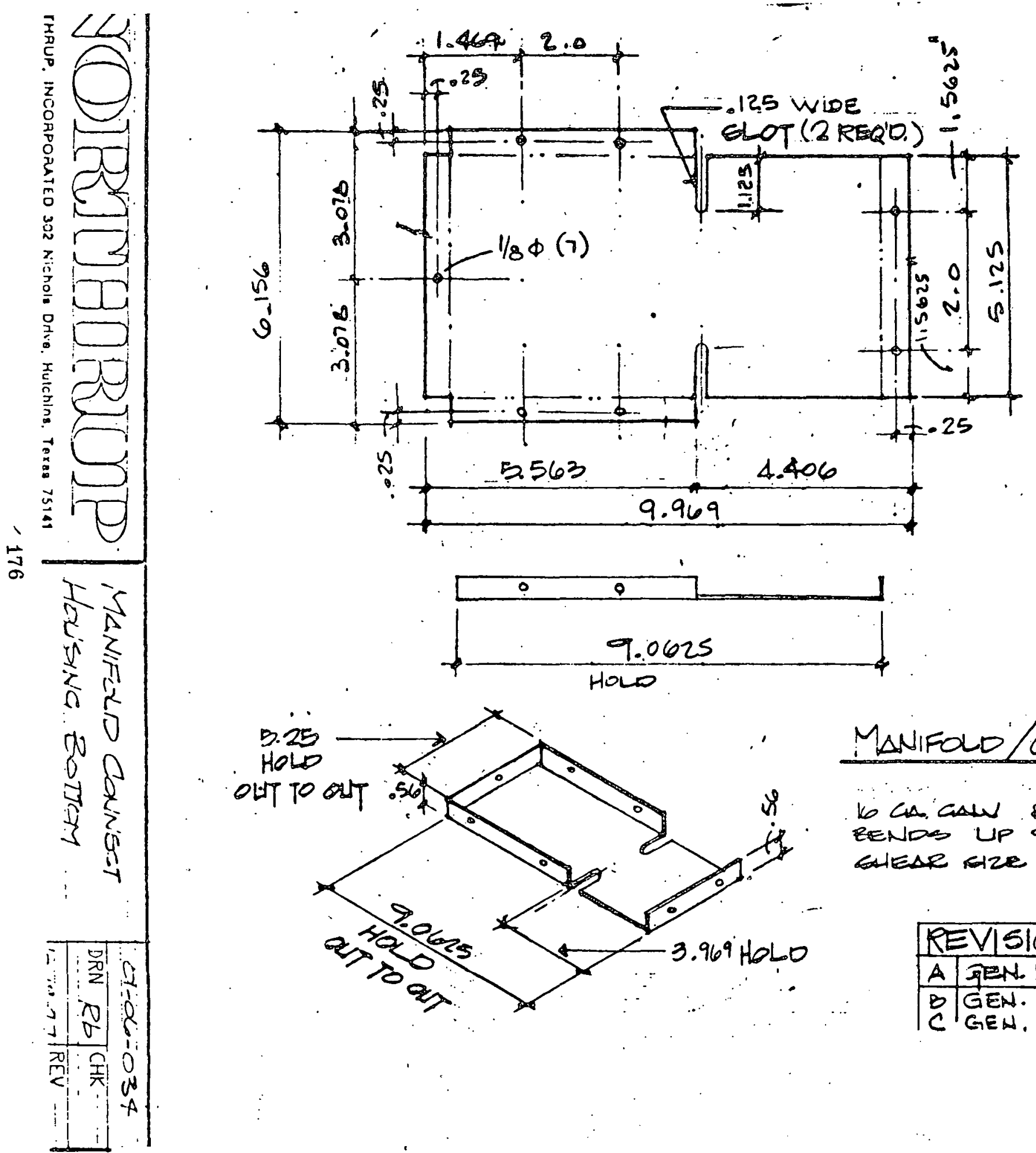

MANIFOLD /CONNECT HOUSING BOTTCM

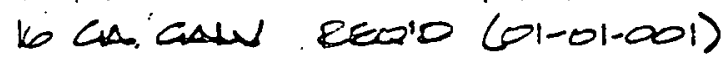

EENDS UP $q 0$

LHEAR fize $60 \times 9.0625$

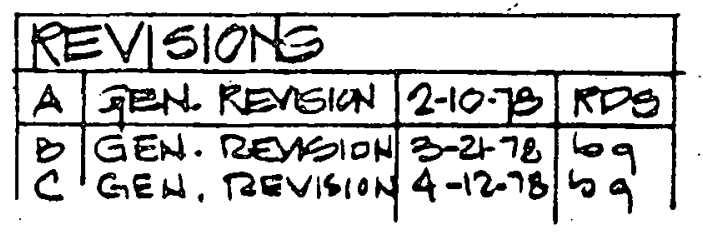




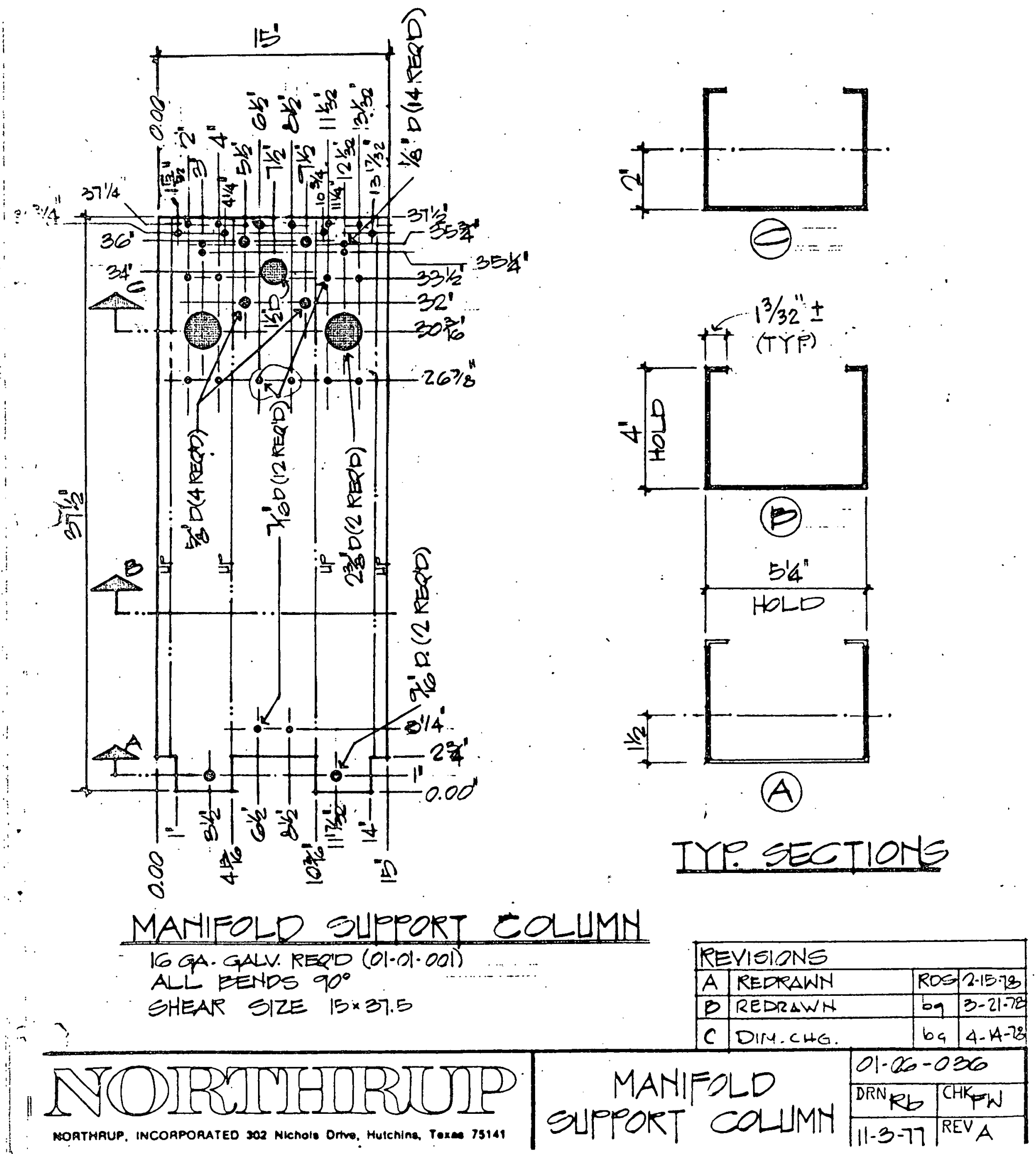


Flanged Units Cast Housing (four iboll)

UCF-2

Set Screw Type

Iformal Duty

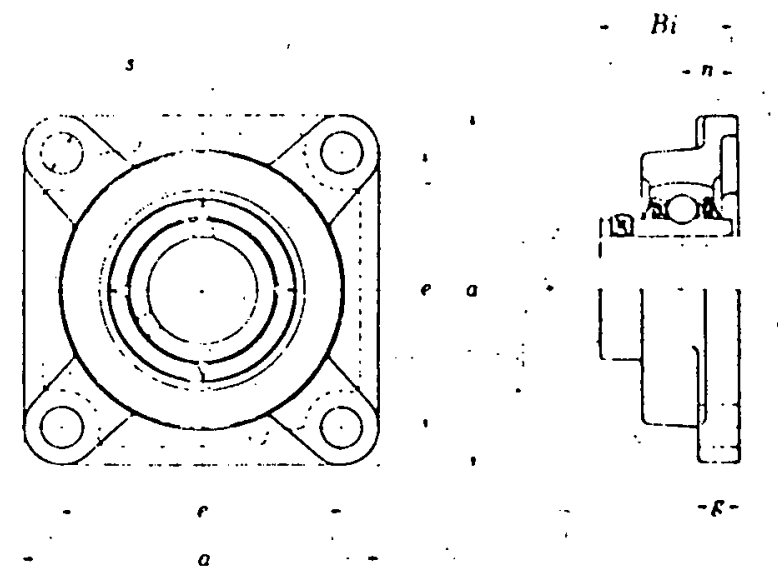

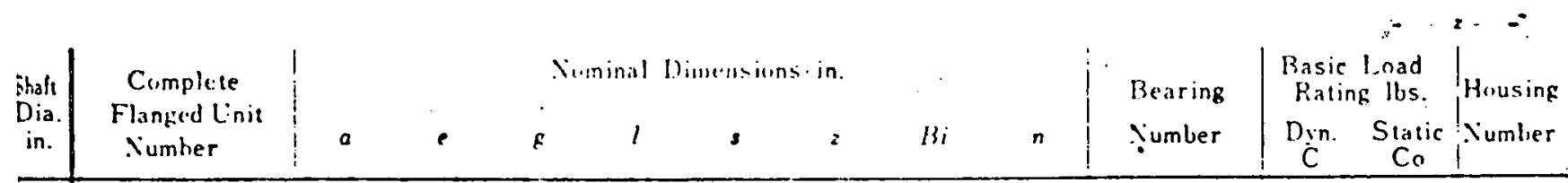

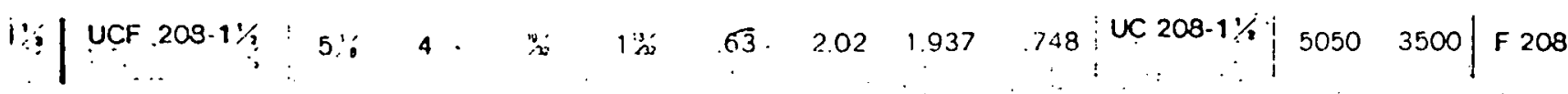

\section{Flanged Units Cast Housing (irour Boli)}

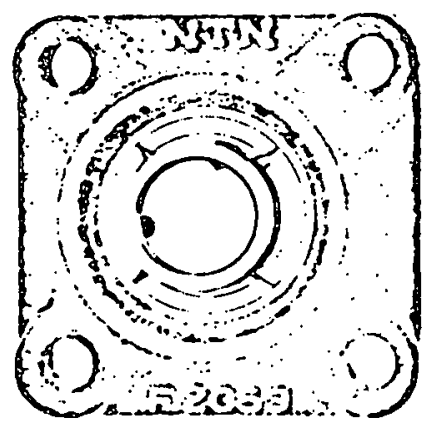

\section{Set Screw}

UCF 2 (for light load)

The housing is square and can be convenicutly secured to, for instance, the side wall of a machine with 4 bolts. Simple in bearing construction and casy to mount, this is the most widely used of all types of units-with-flange. 


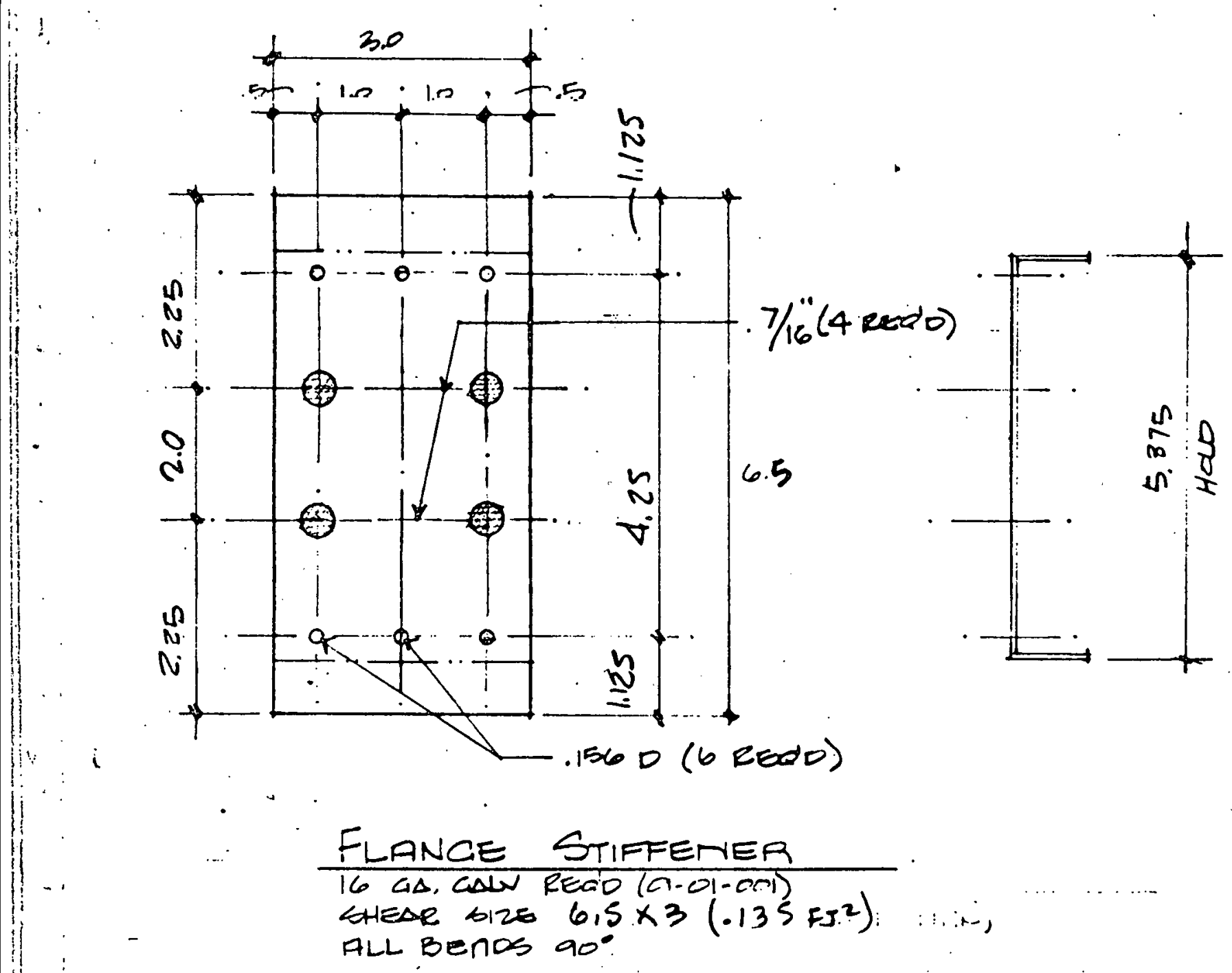



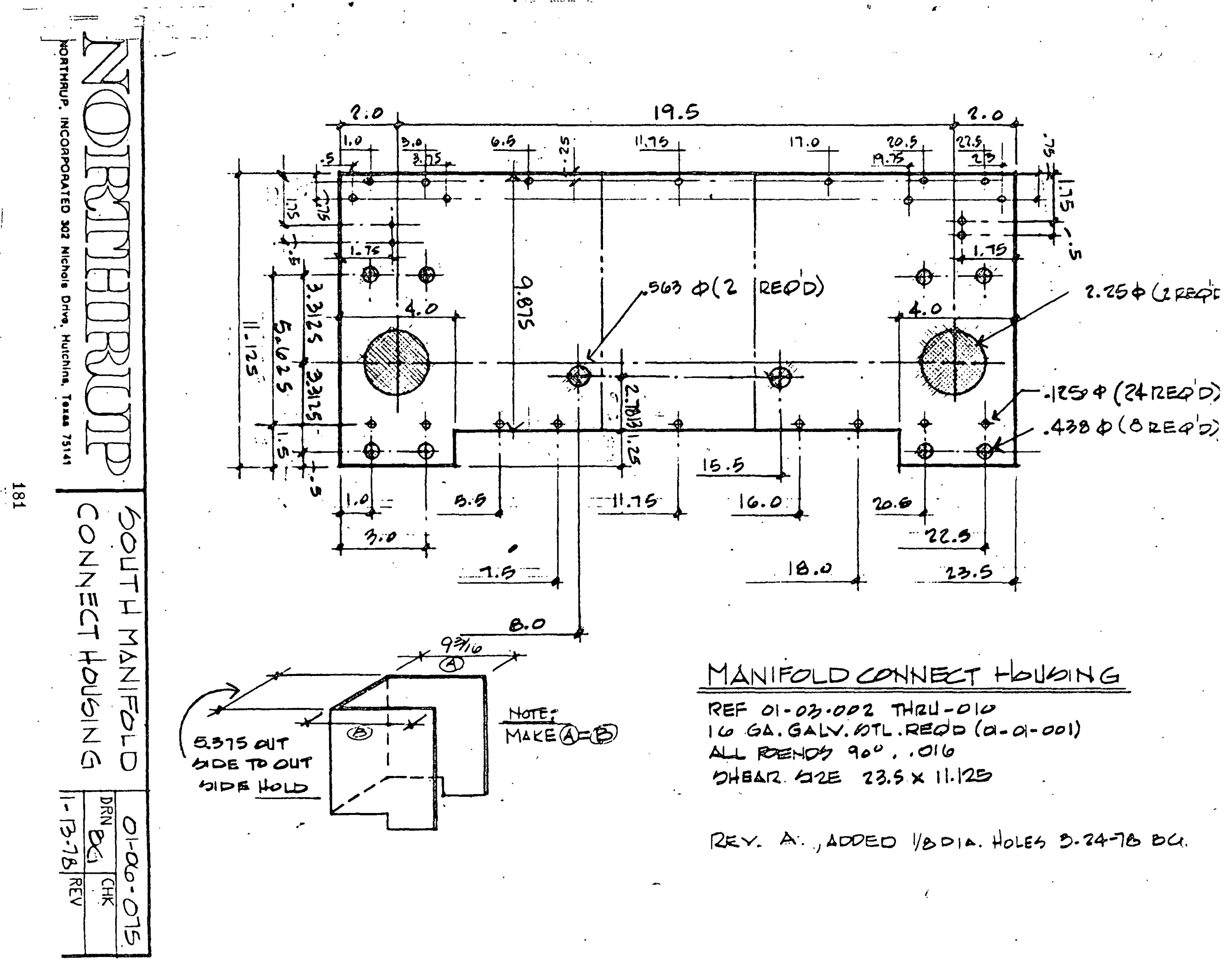

MANIFOLD CONNECT HUVING

REF 01.03.002 THRL-OIO

16 GA.GALV.DTL.REQD $(a-01-001)$

AL $90^{\circ} .016$

SHEAR. SZE $23.5 \times 11.125$

REY. A., $\triangle D O E D$ 1/8DIA. HOLES $3.24-78$ BC. 

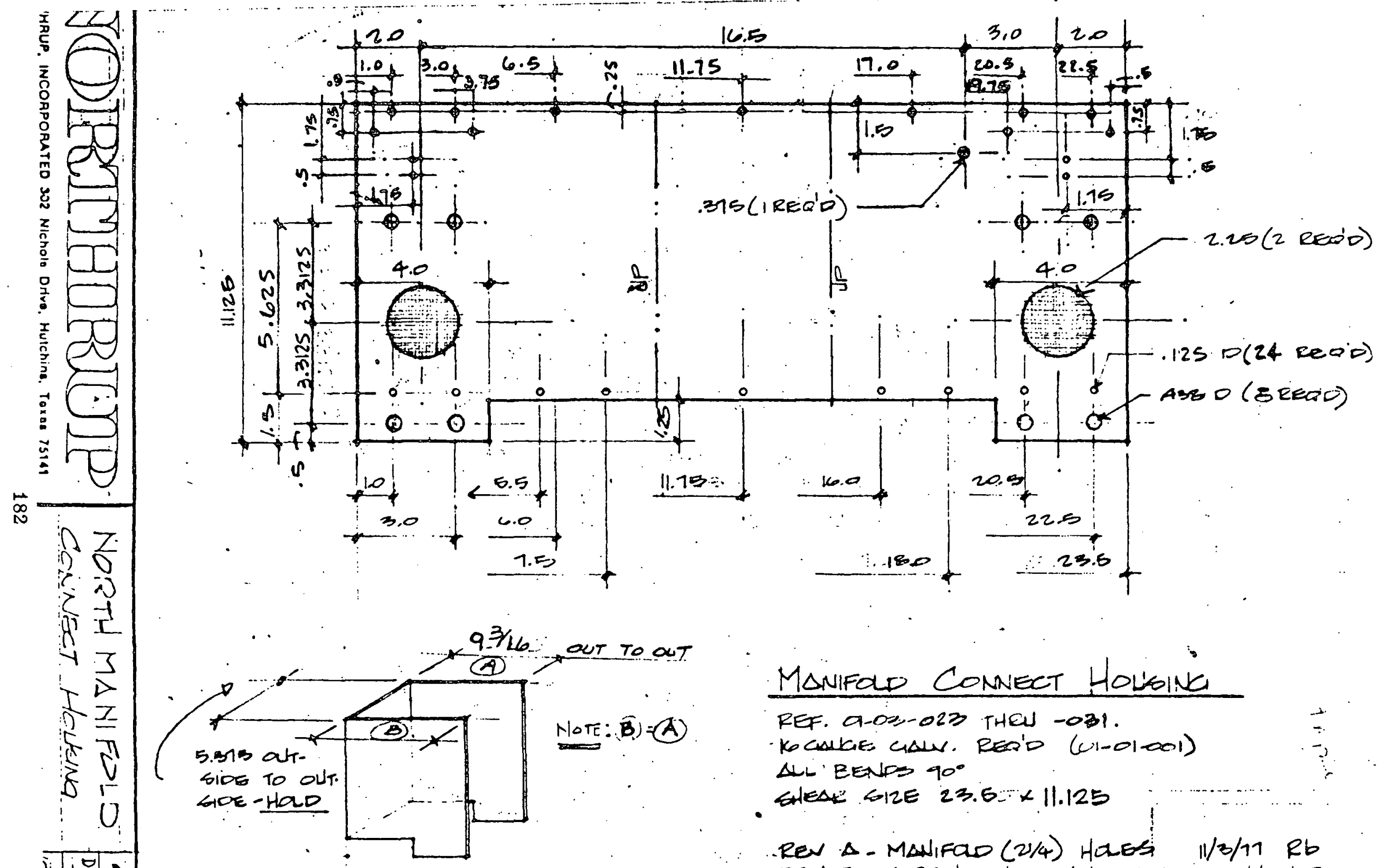

Manifar Connect Hovinin REF. O-Da-023 THES -OB!.

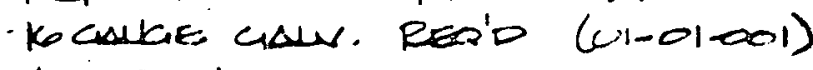
$\Delta L$ LENTS $90^{\circ}$ SHEA: CO12E $23.6 \times 11.125$ Rev $\triangle$ - MANIFAO (2//4) HaWes 11/3/11 Rb REV B. SPEC'O HORTH MANIFULD 11/15/77 HUC. KEV C- BLANK SIZE REV. 2.10.78 RDS REV. D - $\triangle D D E D$ I/B DIA. HOLE' 3-24-7B BCA 

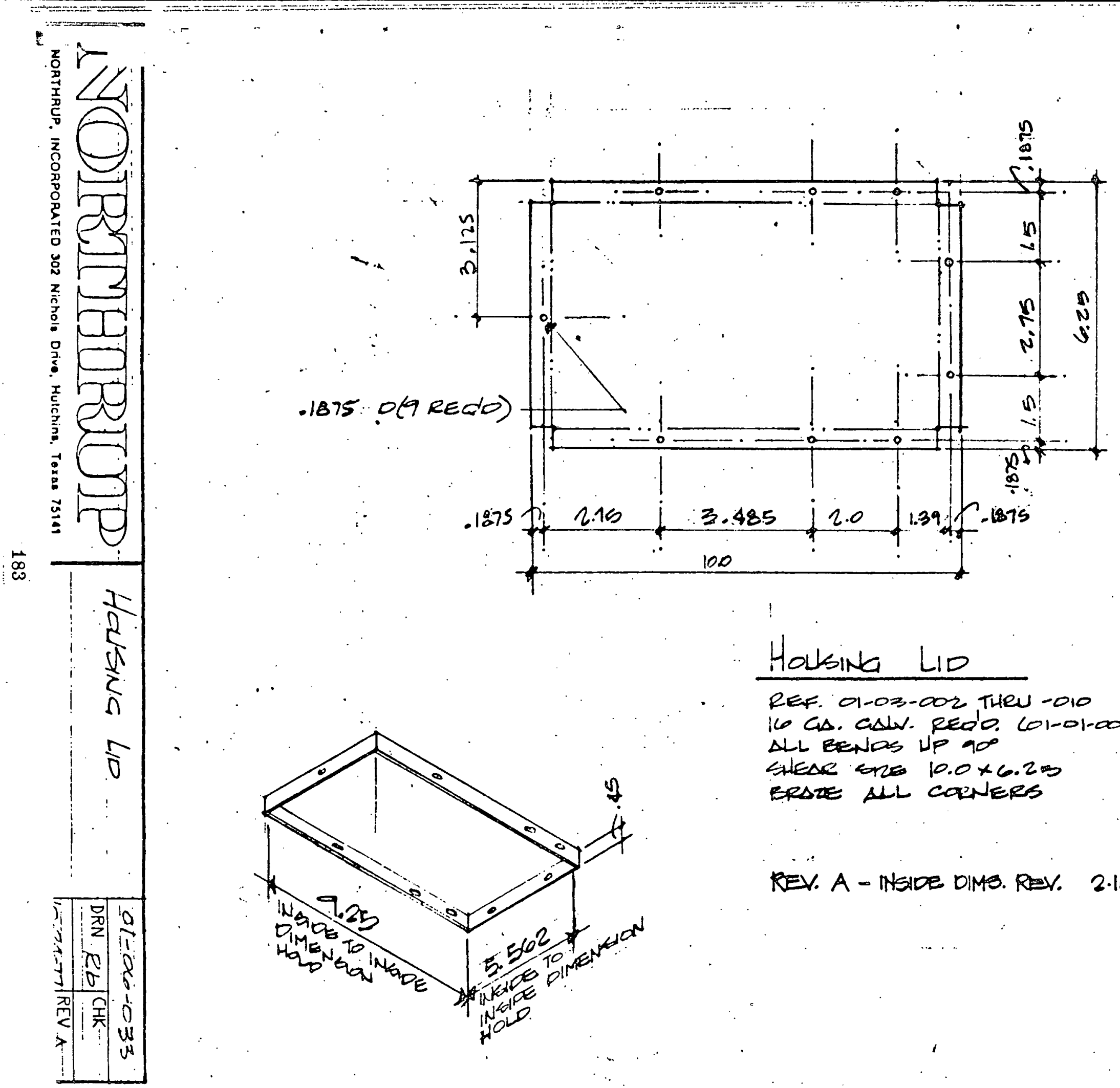

Holkina Lio

REF O1-03-002 THRU -010

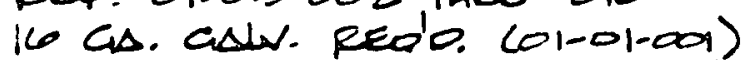
$\triangle L L$ ERNAS UP $90^{\circ}$ SheAR otze $10.0 \times 6.25$ BRATE ALL COUNERS

REV. A- NHSIDE DIMS. REV. 2.10.78 RDS 


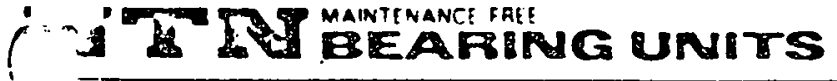

Flanged Units Cast Housing (Four Bolt)

\section{UCF2 \\ Set Screw Type}

$\left.\left[\begin{array}{c}0 \\ 0 \\ 0\end{array}\right]\right]^{\text {Normal Duty }}$

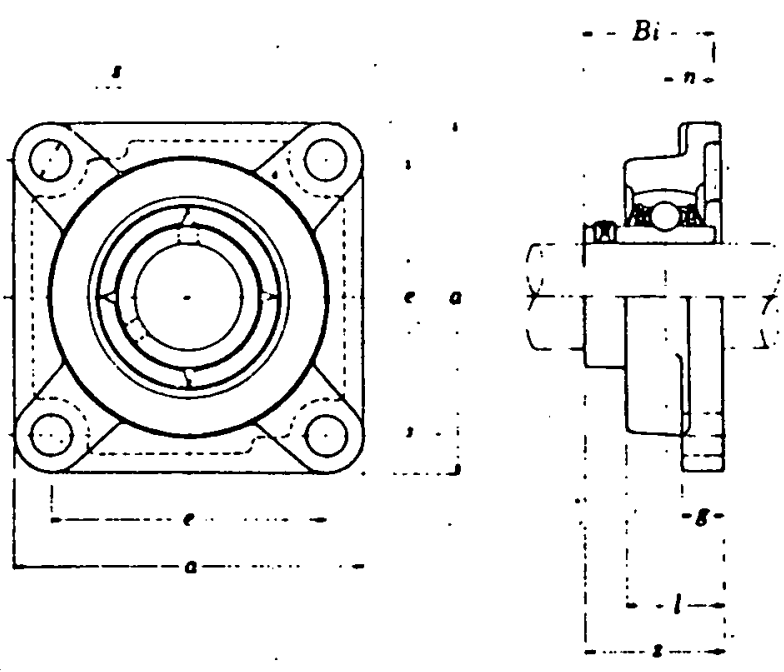

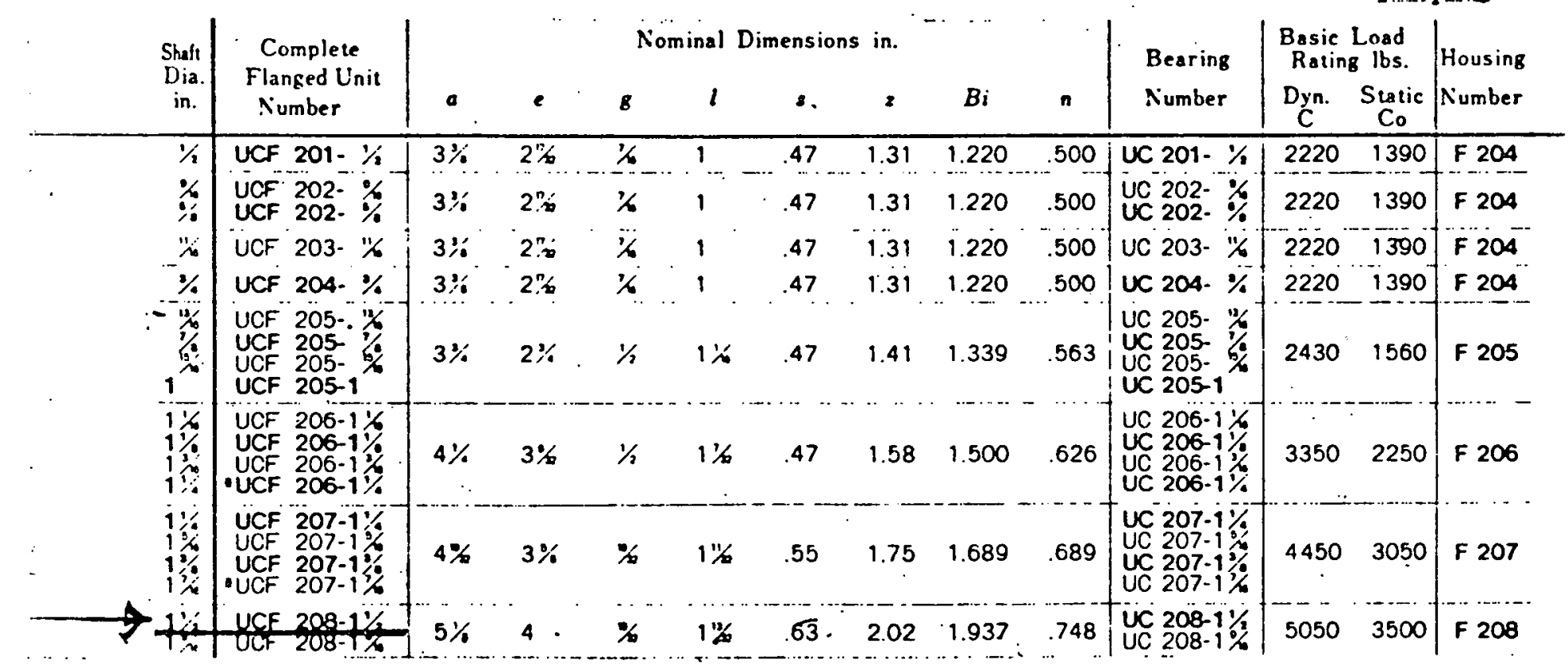

Bearina/Holoina UnIT

REFER A-OSNozi THRL a-0300 FeaM NTN CATALOC 2596 


\section{MECHANICAL}

\section{-F. Solar system}

1. GENERAL:

This Section valid only when considered in total with other contract Documents. Cross references are for convenience of reader and their inclusion in or amission from any particular section

in no way limits scope of particular section or intent of any contract Document.

2. WORK SPECIFIED HEPEIN:

A. Complete installed solar collection system as shown on the drawings.

B. Erection, and connection of solar collectors and oollector support systens.

3. REJATED WORK:

Specified in other Sections:

A. Electrical power wiring and conduit (in Electrical Division).

4. Matertals \& DQuiPMENT:

A. Solar collectors and Structural supports:

1. Solar collectors:

Each collector consists of fresnel lens (12" wide x $1161 / 2$ " net apeture), insulated housing, selective coated copper absorber tube with hollow end shafts and 1/4" MPT connections through each shaft to the absorber tube. Components of each collector consist of the following:

a. Factory assembled housing, interior insulation, end (north and south) $11 / 4$ " O.D. hollow shafts, absorber tube, 1/4"

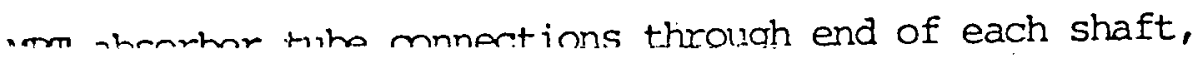




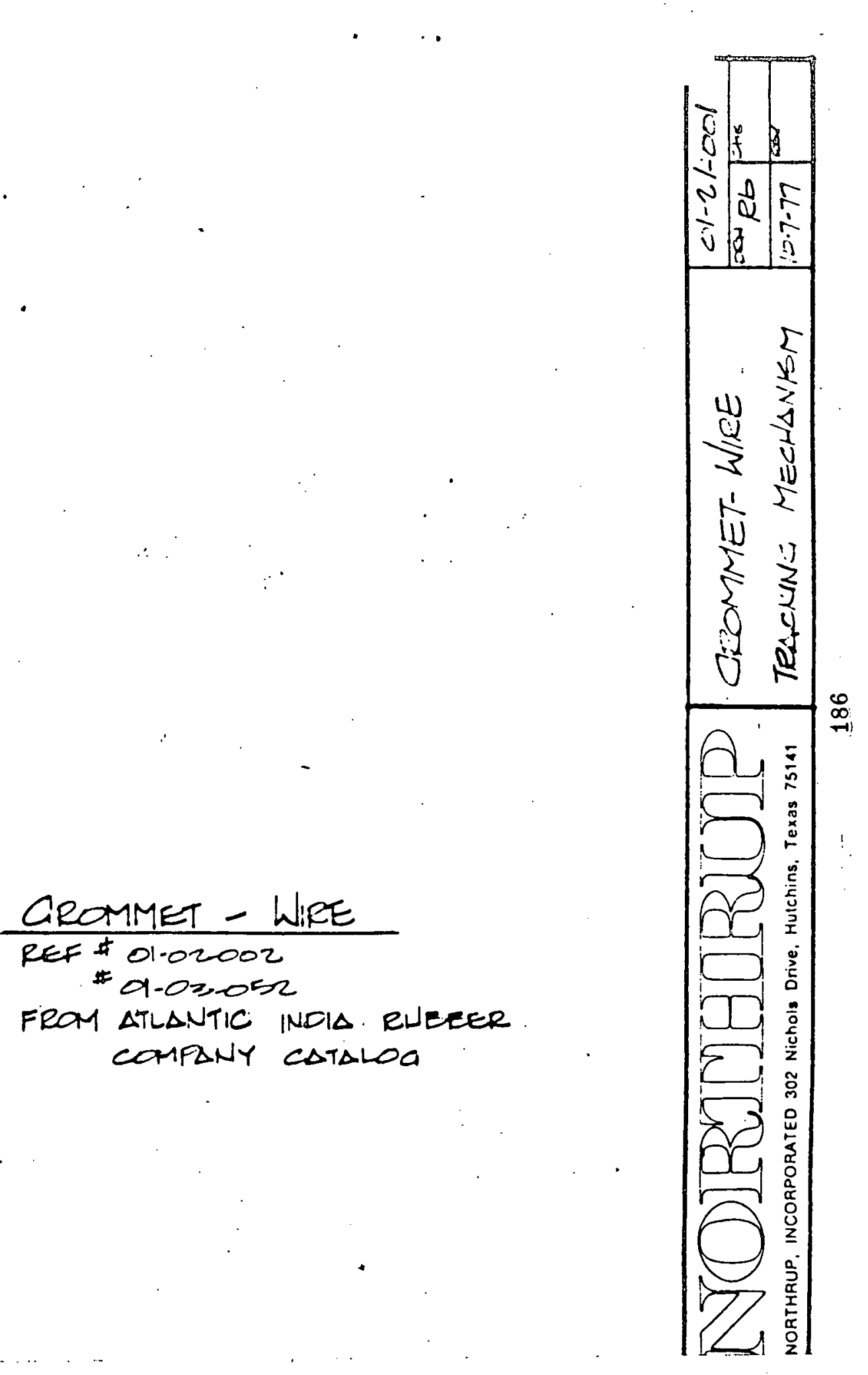




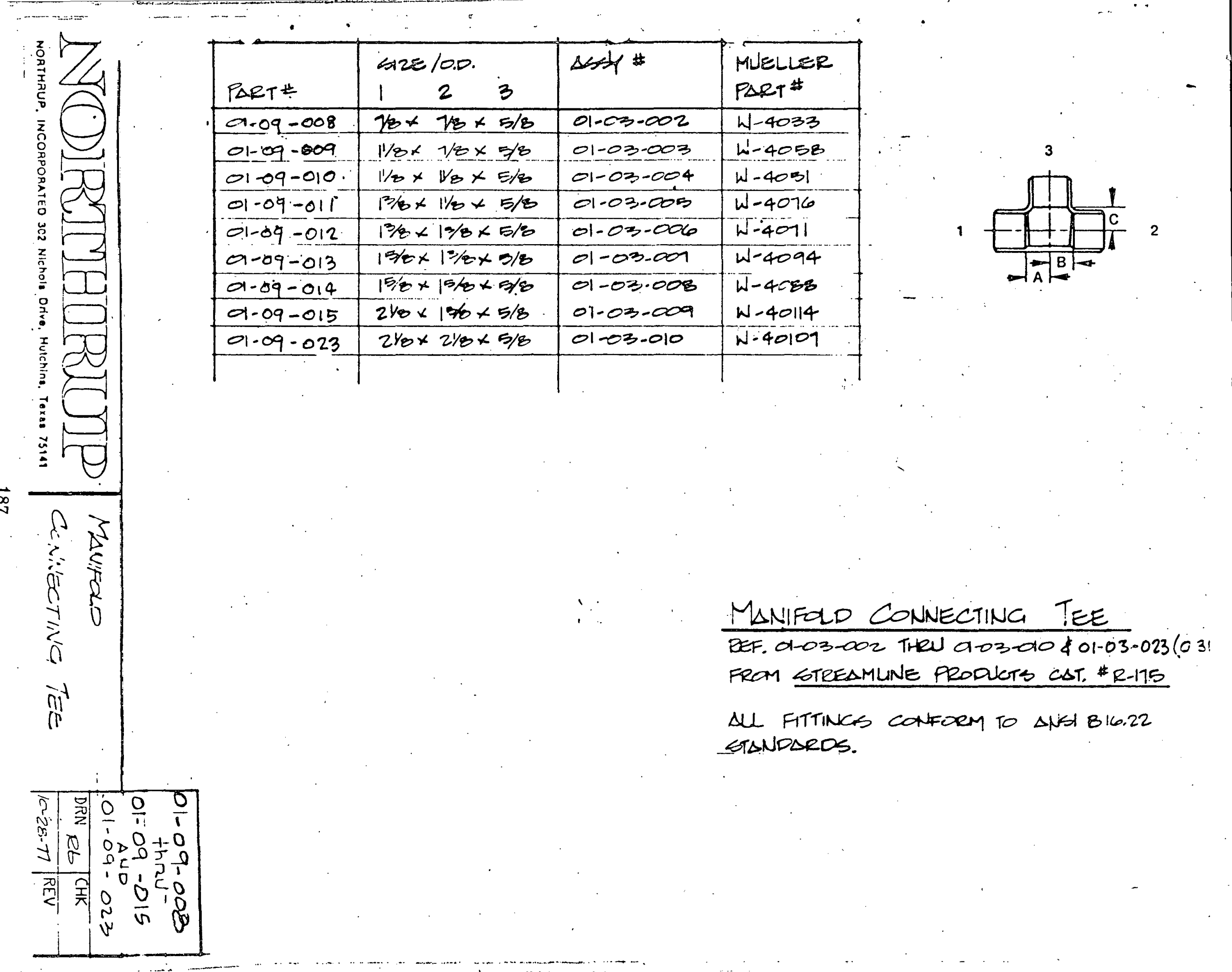




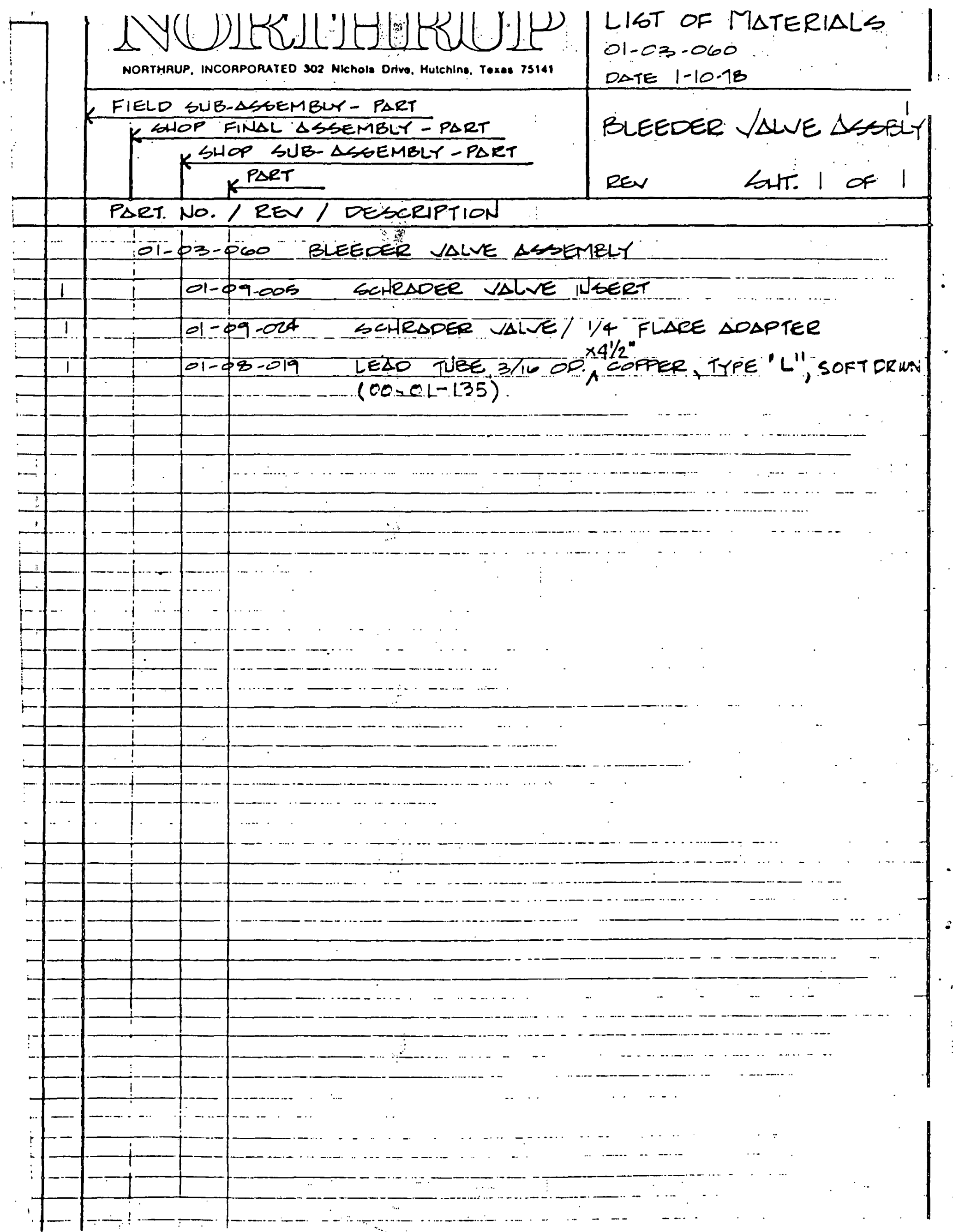




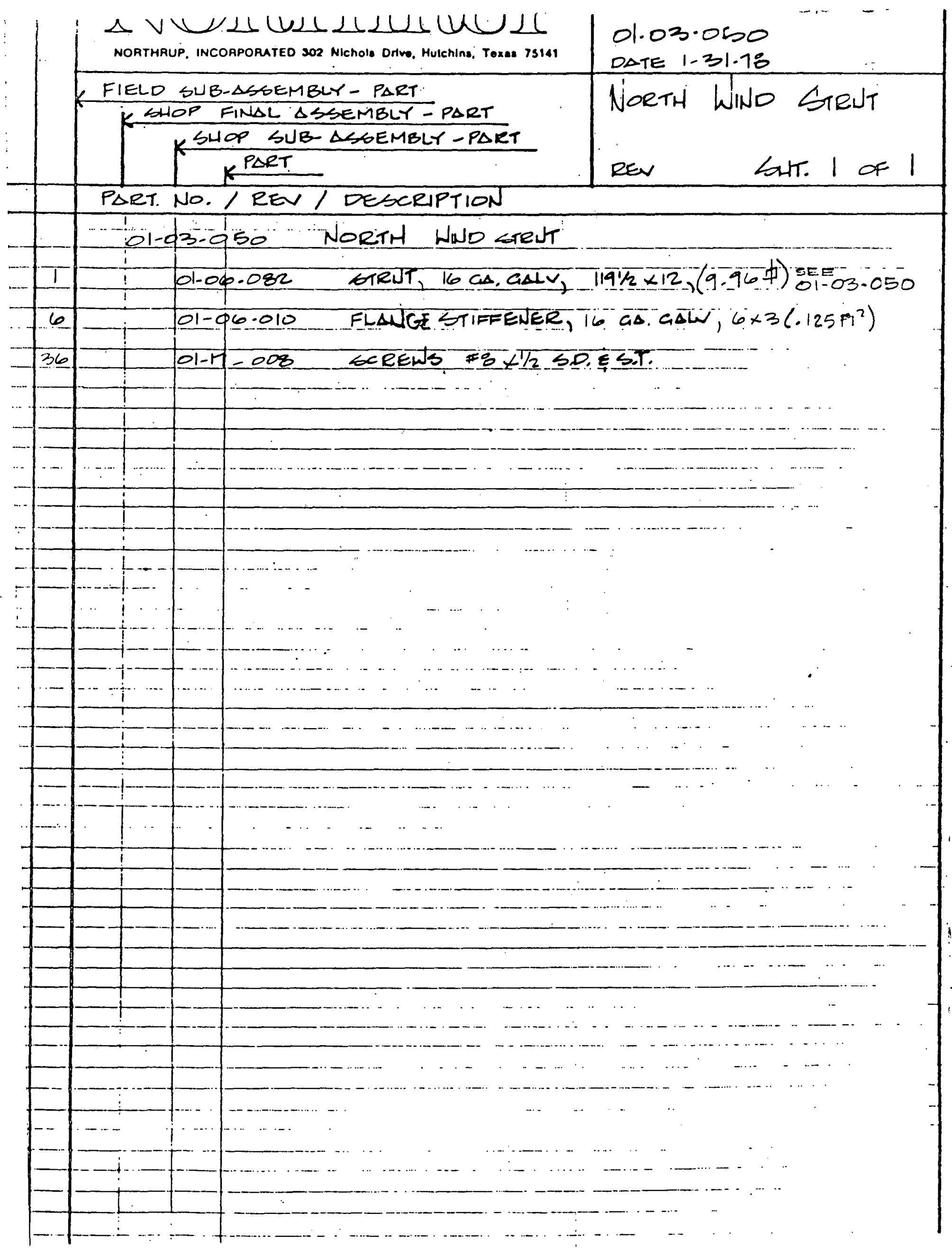

190 


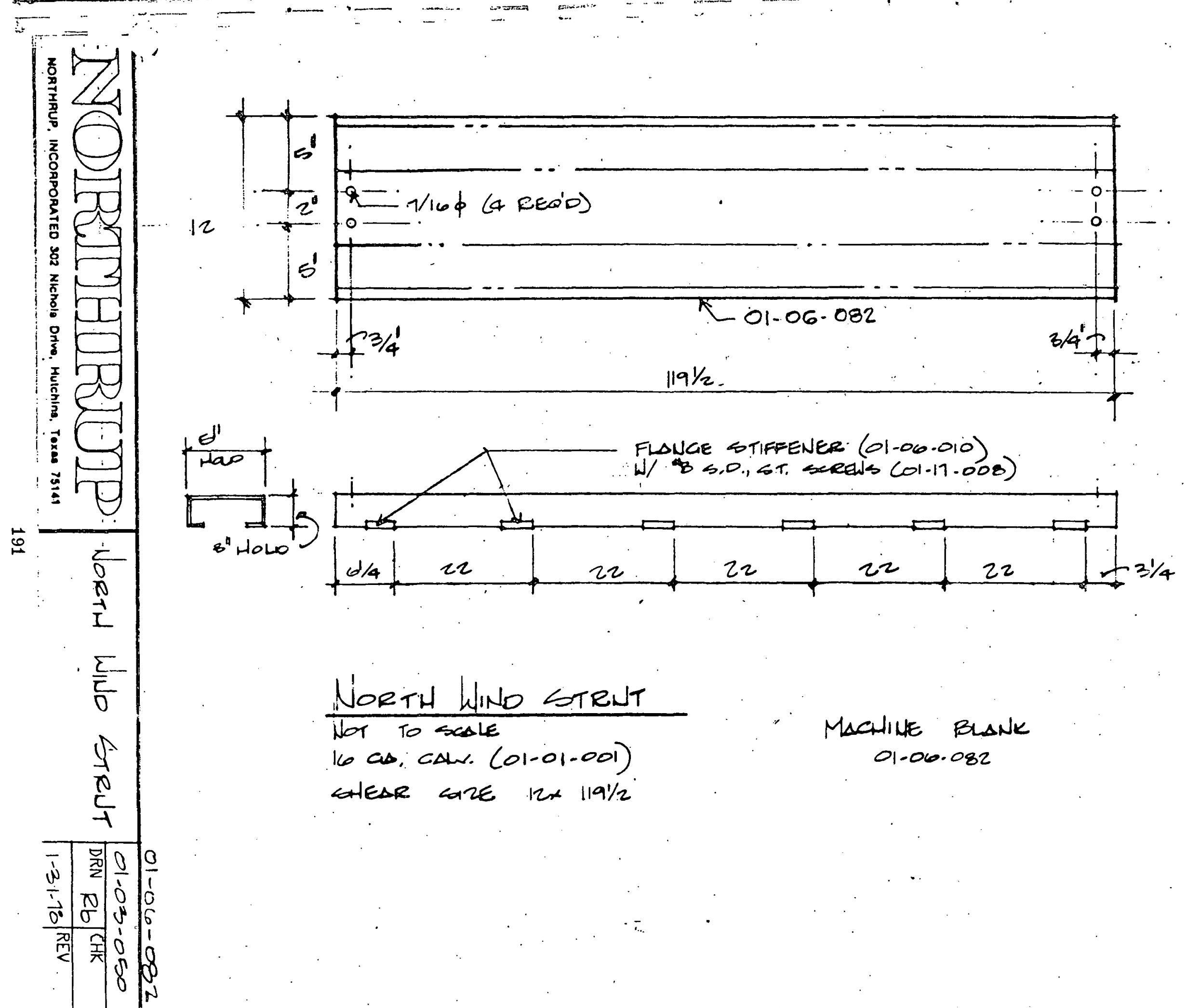




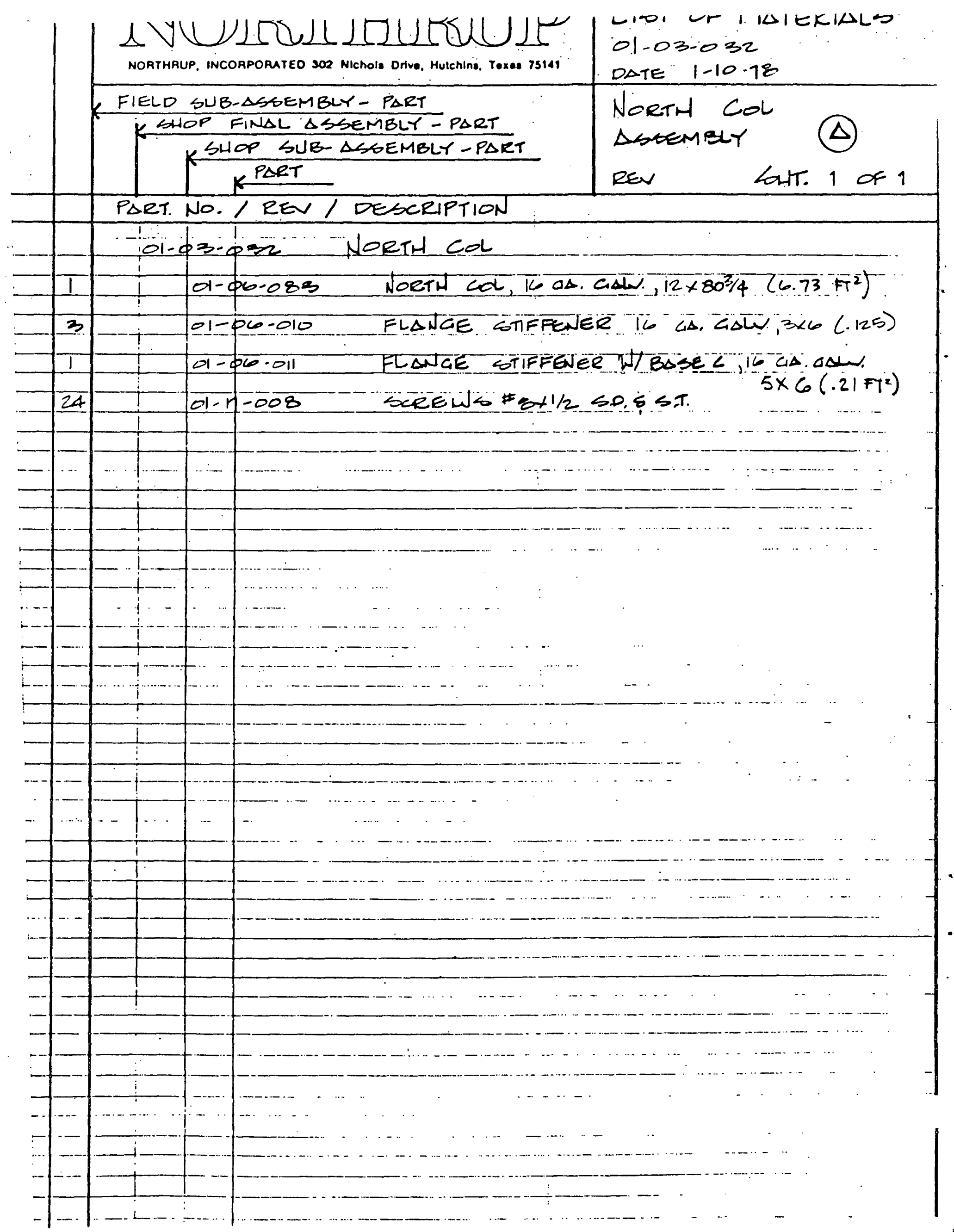




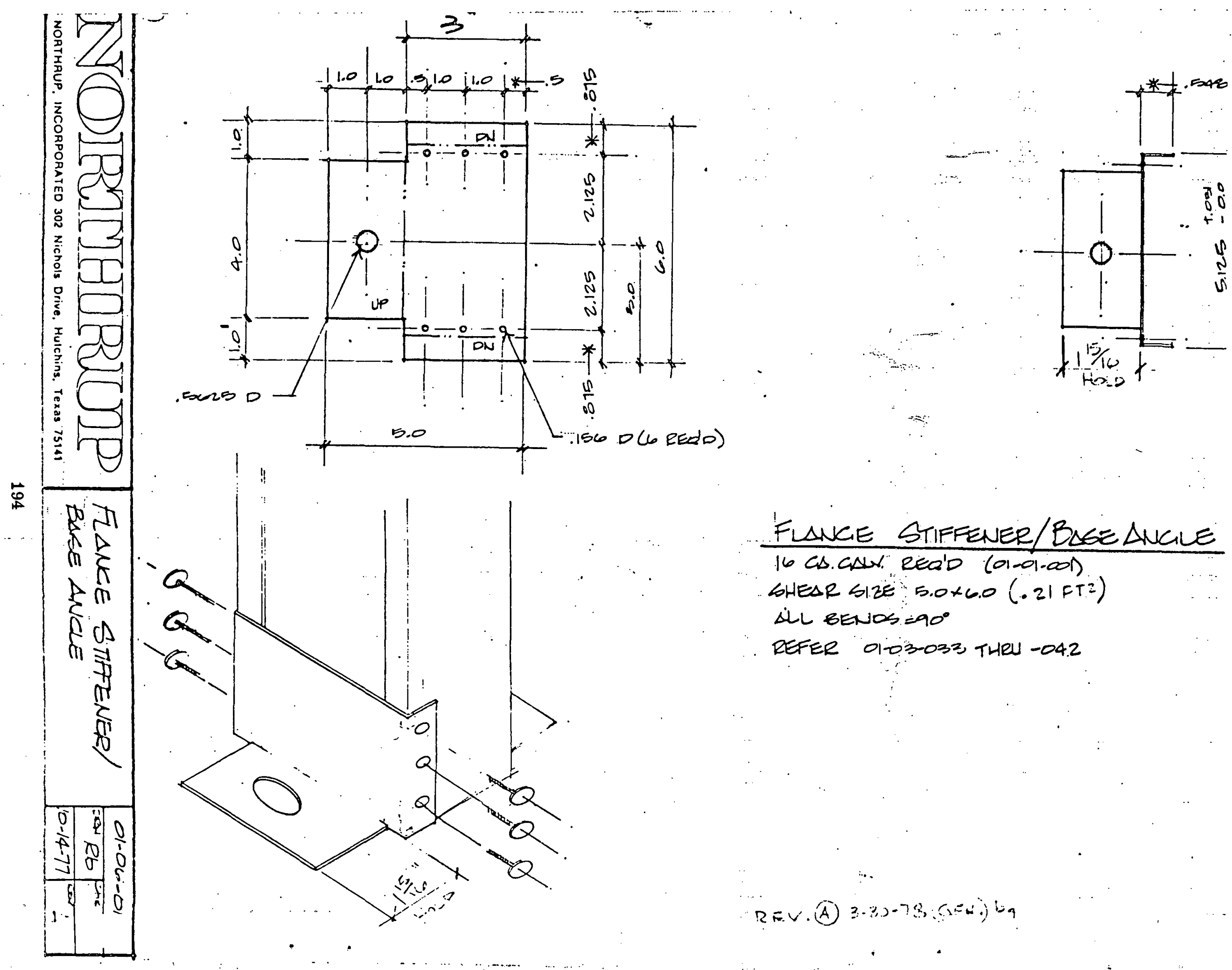


This page left blank 


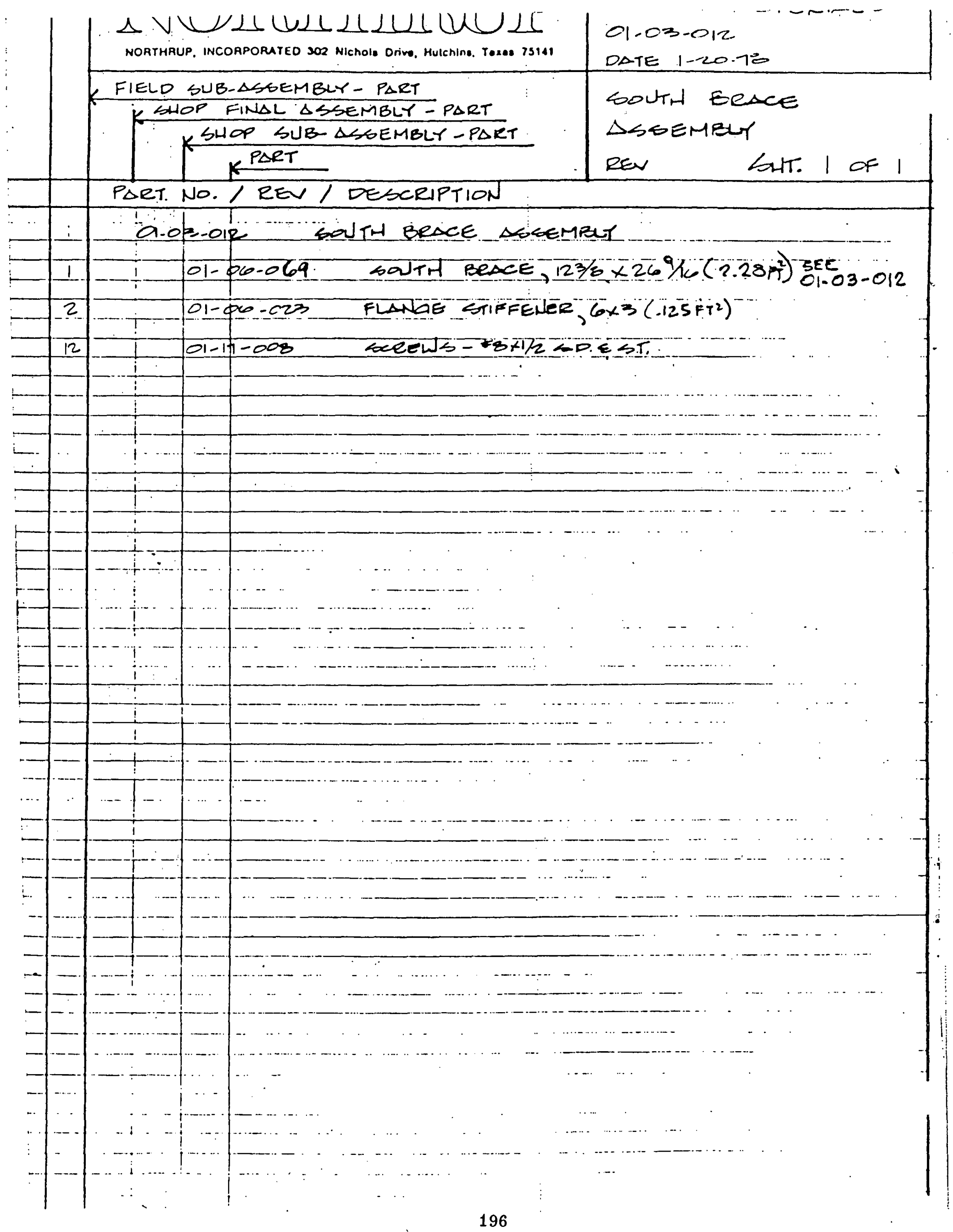



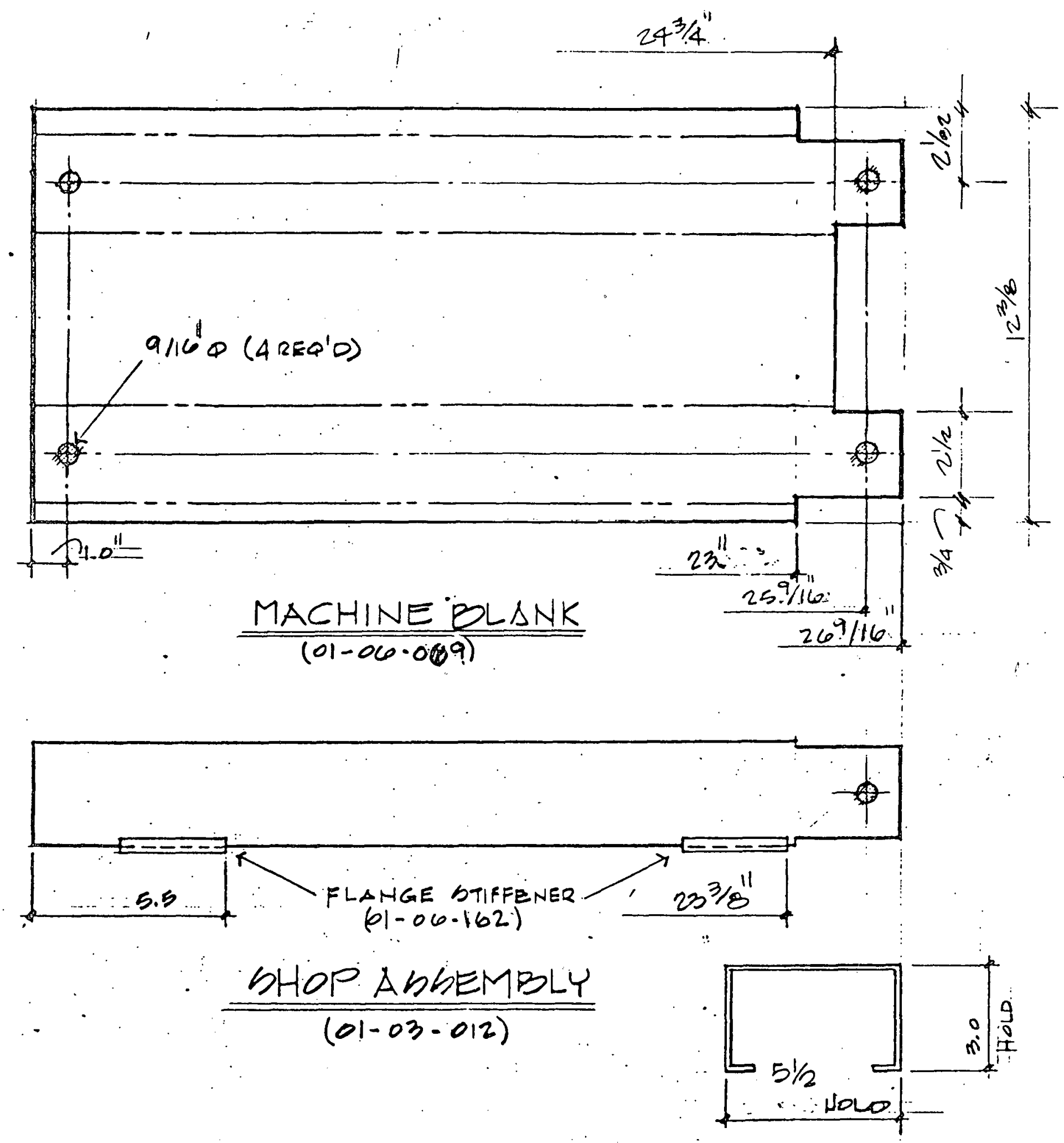

$\frac{M \triangle T E R I \Delta L}{16 C \Delta \cdot C \Delta W . R E O^{\prime} D(01-01-\infty 01)}$

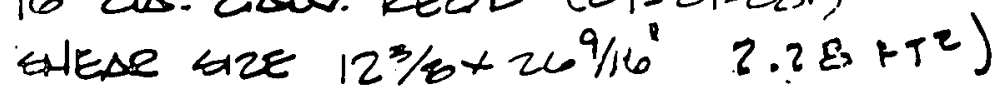

REN $\triangle$ IEEDESICN $1-26-78$. Rb

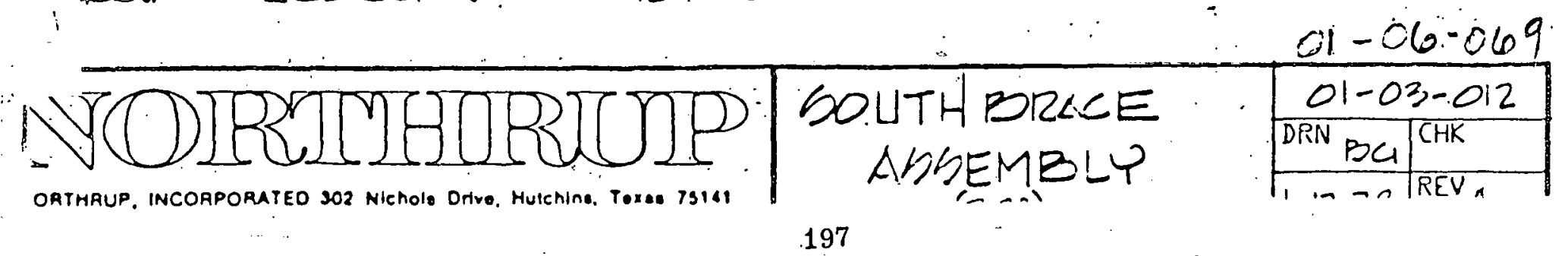



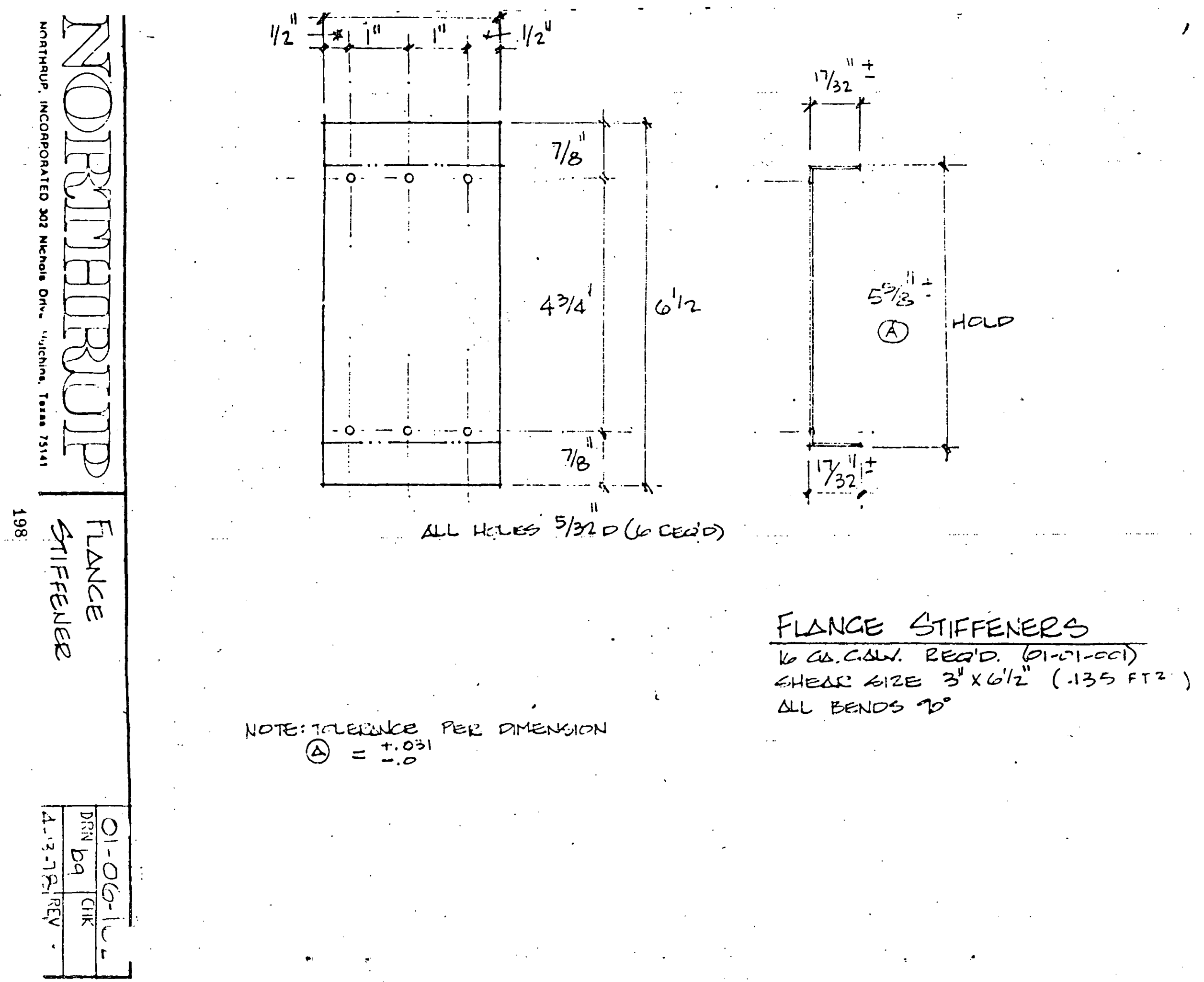

NOTE: TRLELINGE PER DMENENION (A) $= \pm .03$ 


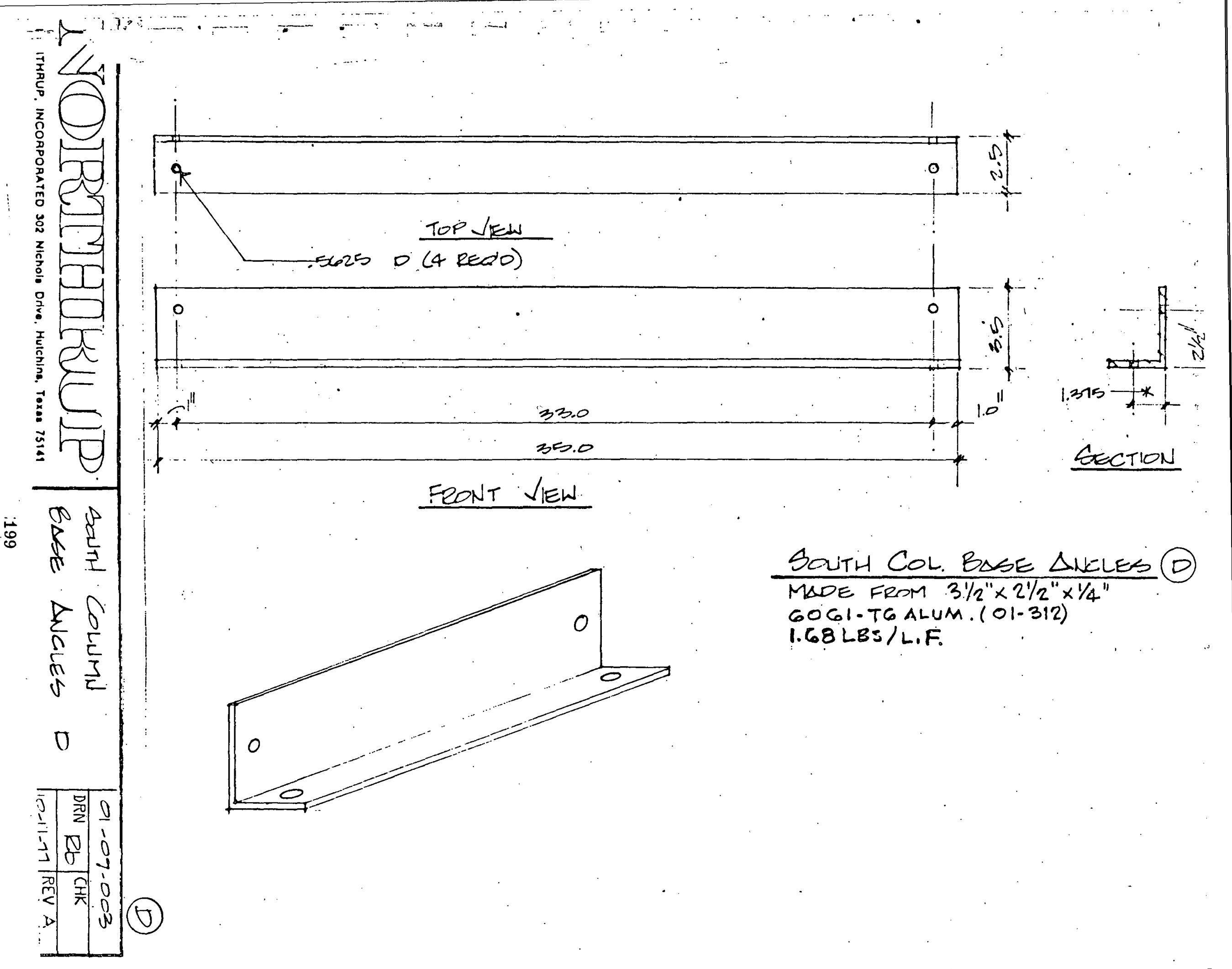




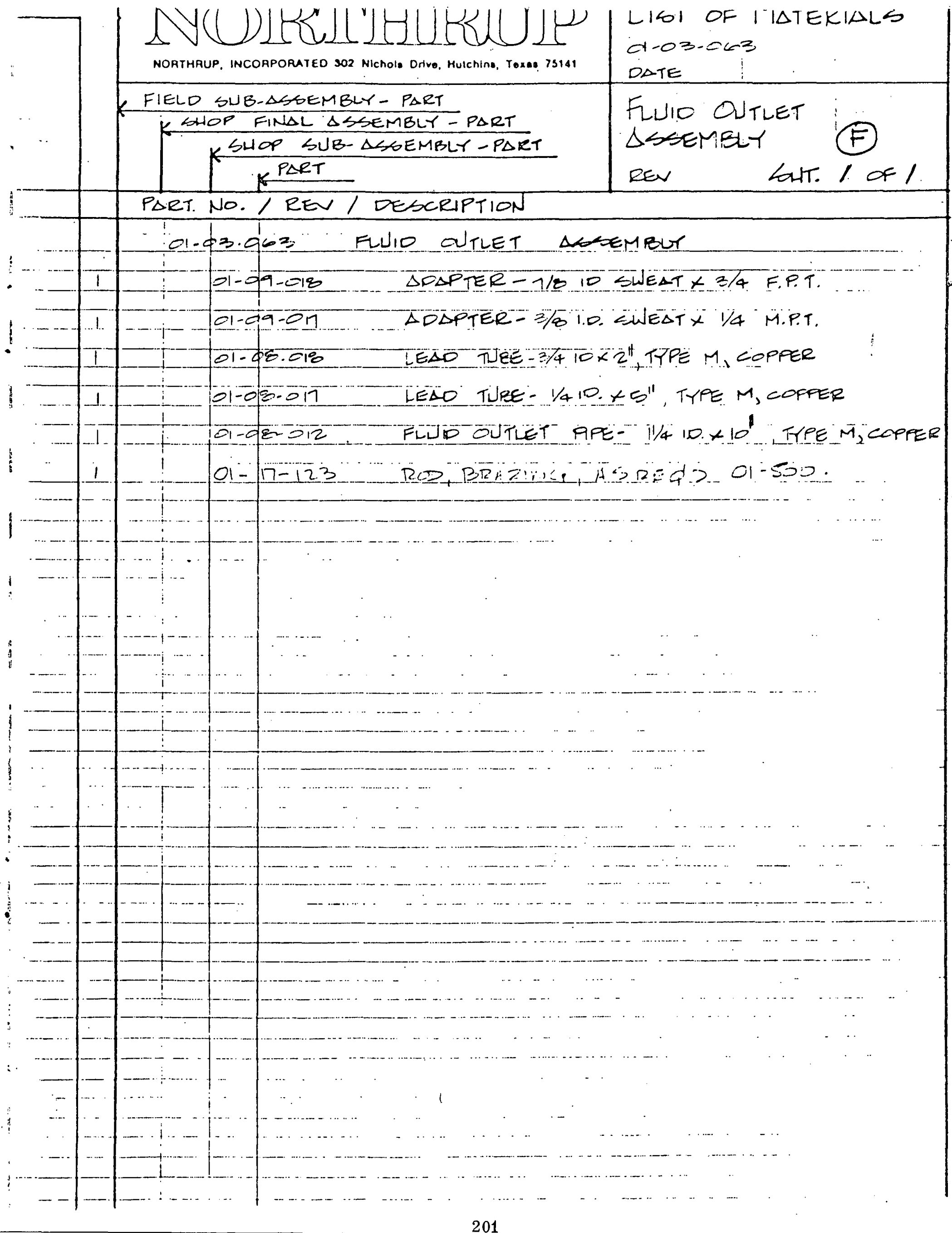



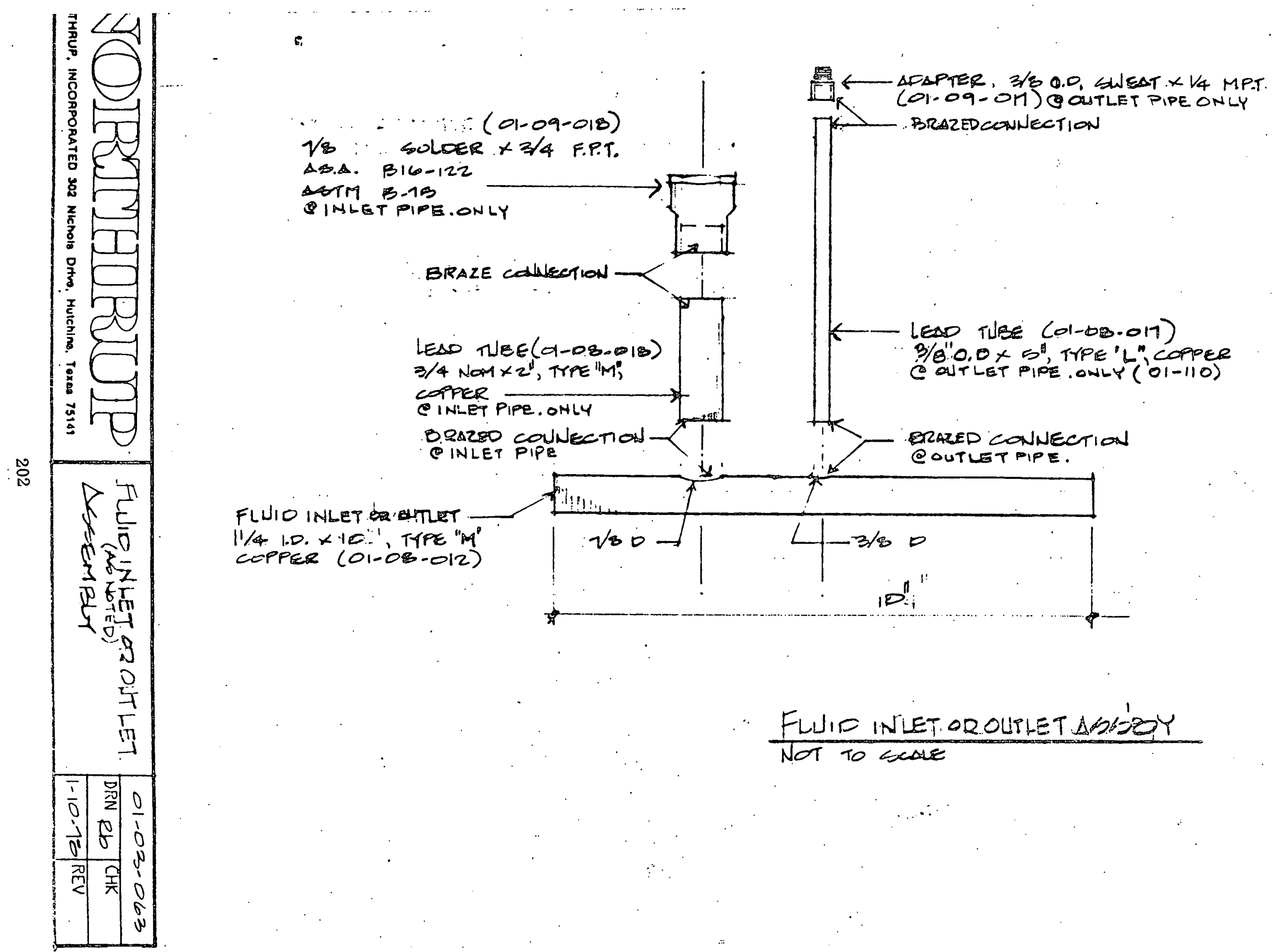
REF DRWa/LHT \# a-03.063 FOR WORK THKS SHEET

AV(l)



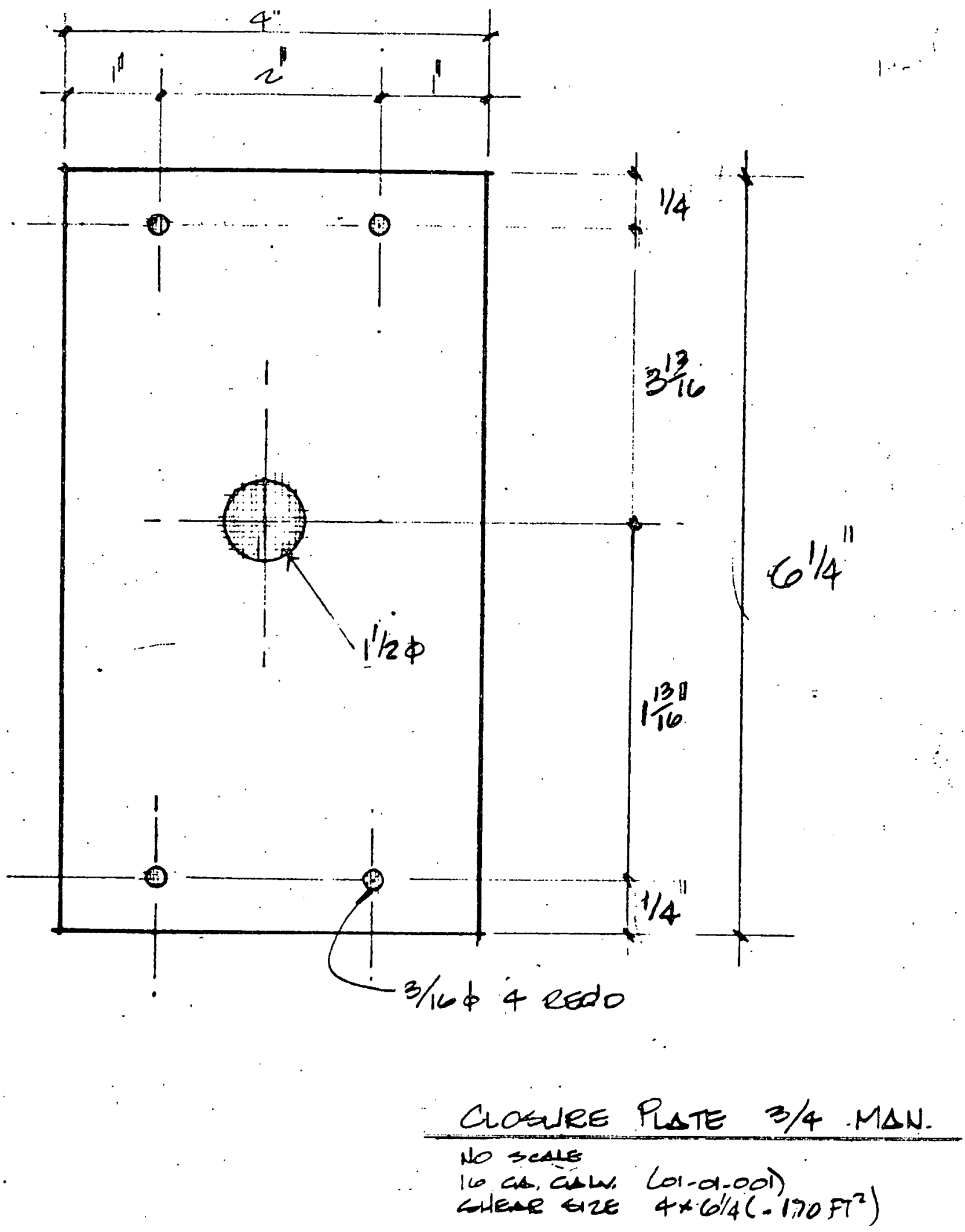

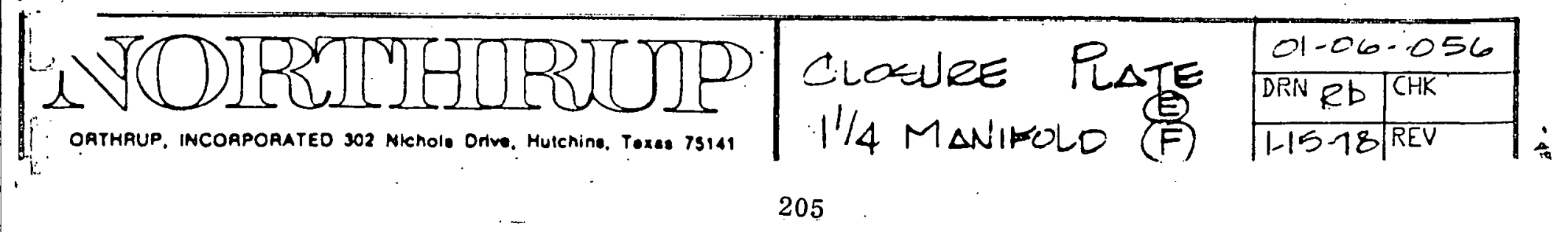




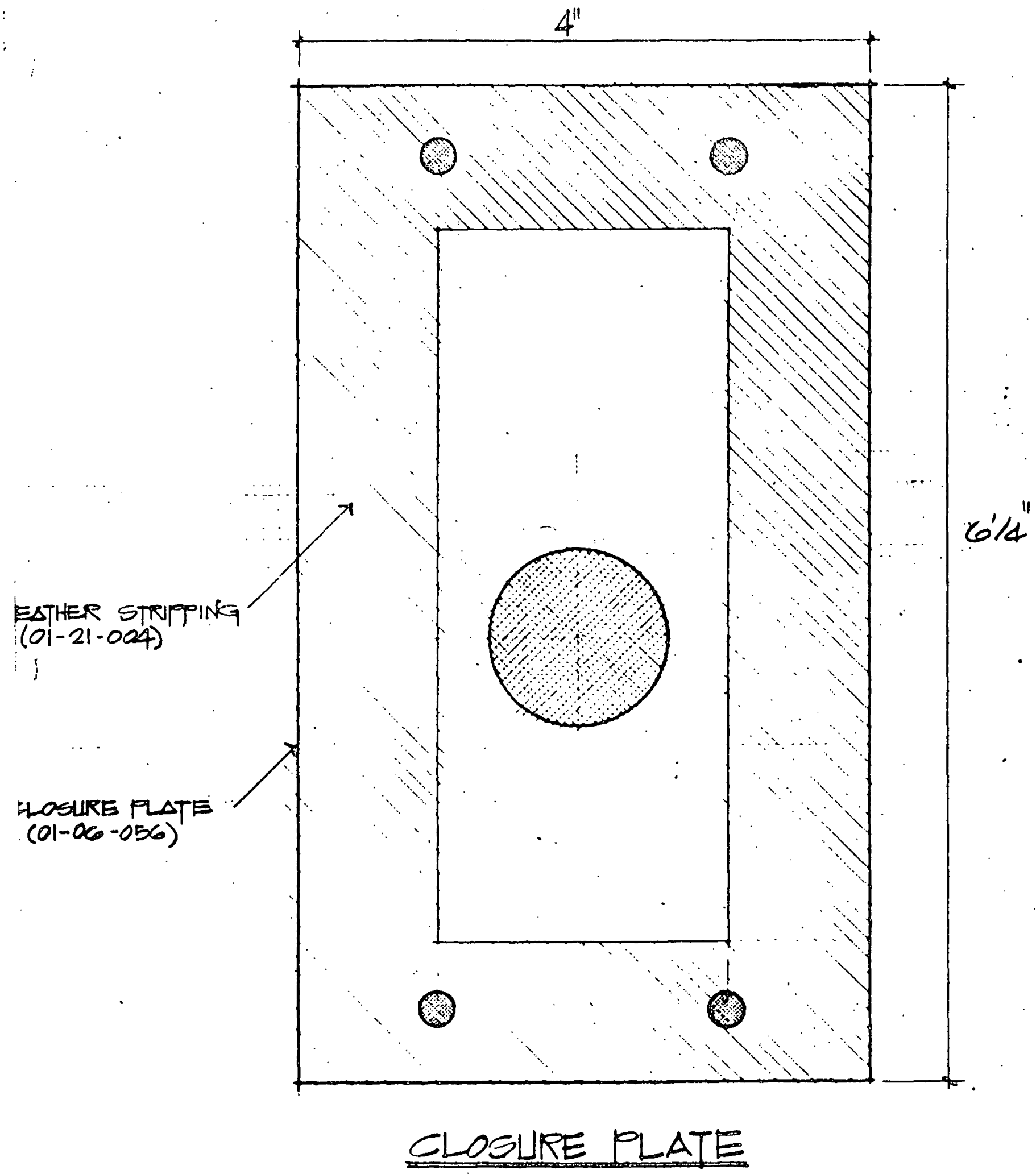

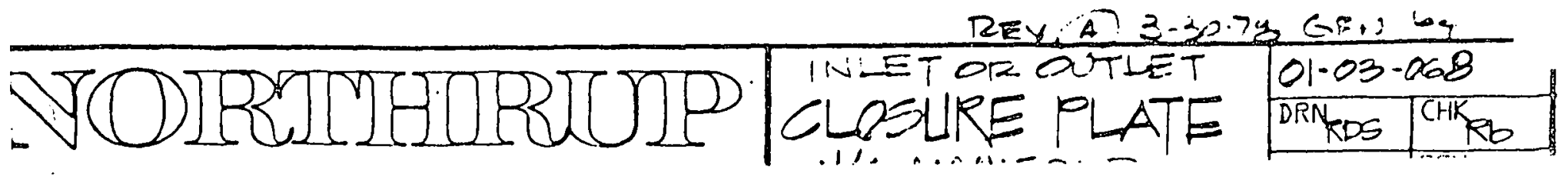




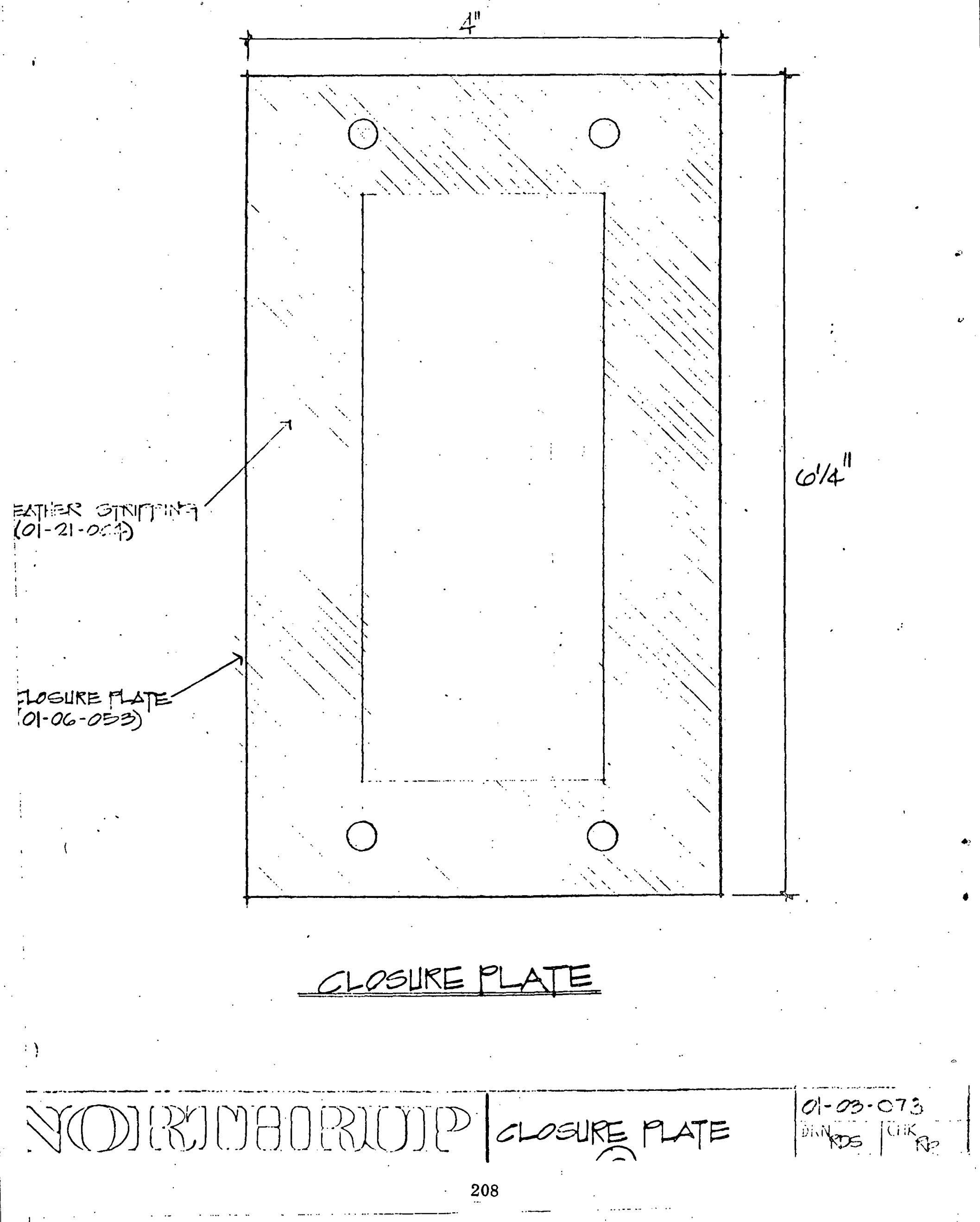




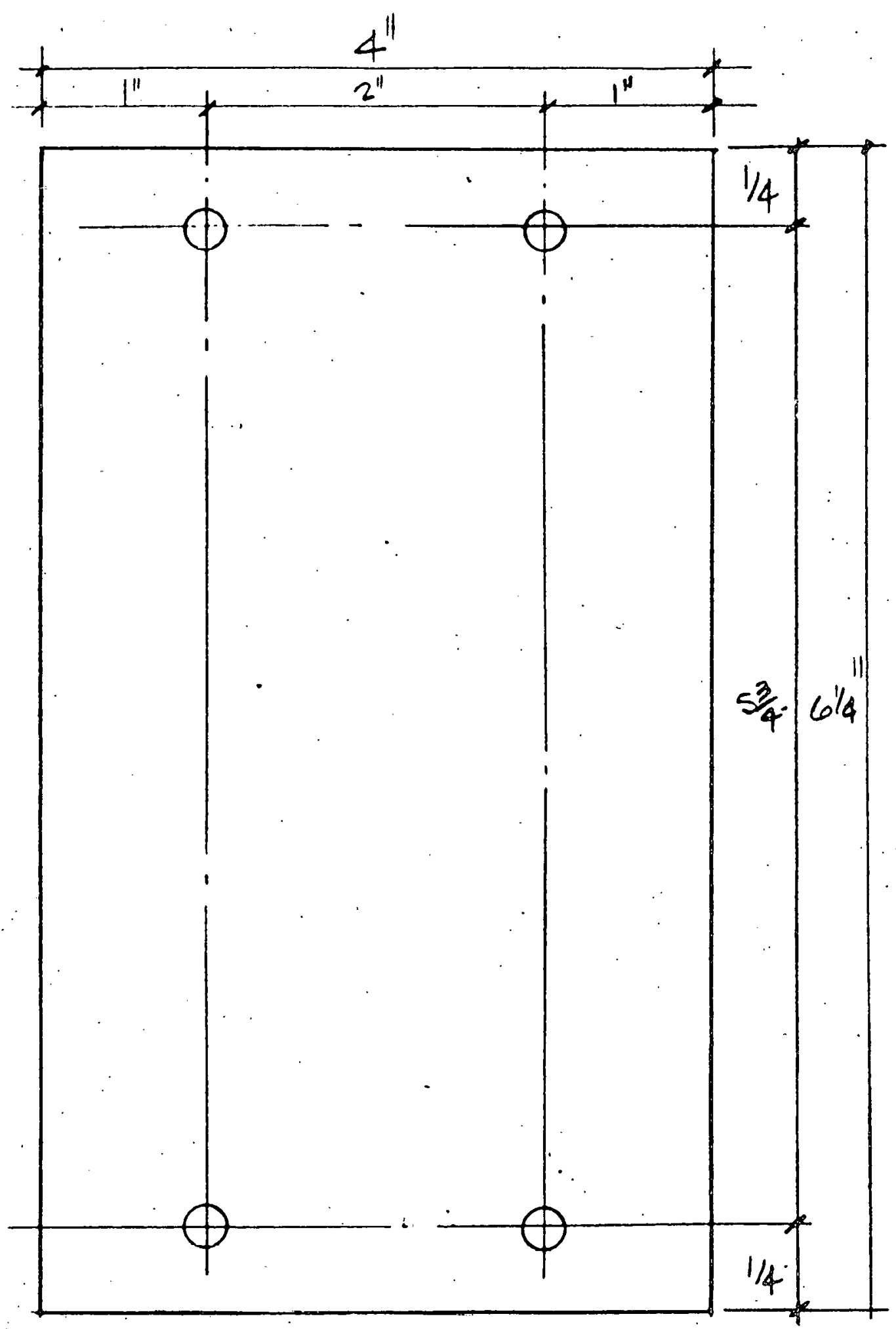

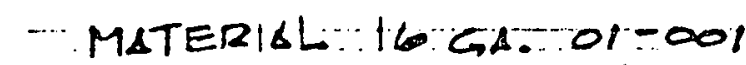
$=4 \times 61 / 4$ (. $\left.170 \% T^{2}\right)$

i. ....

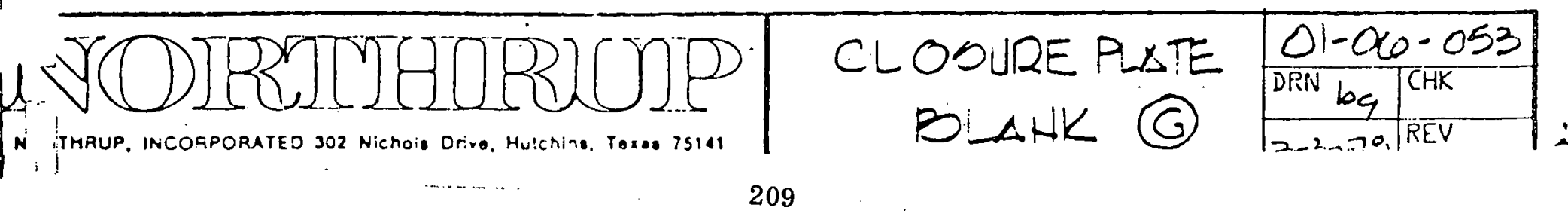



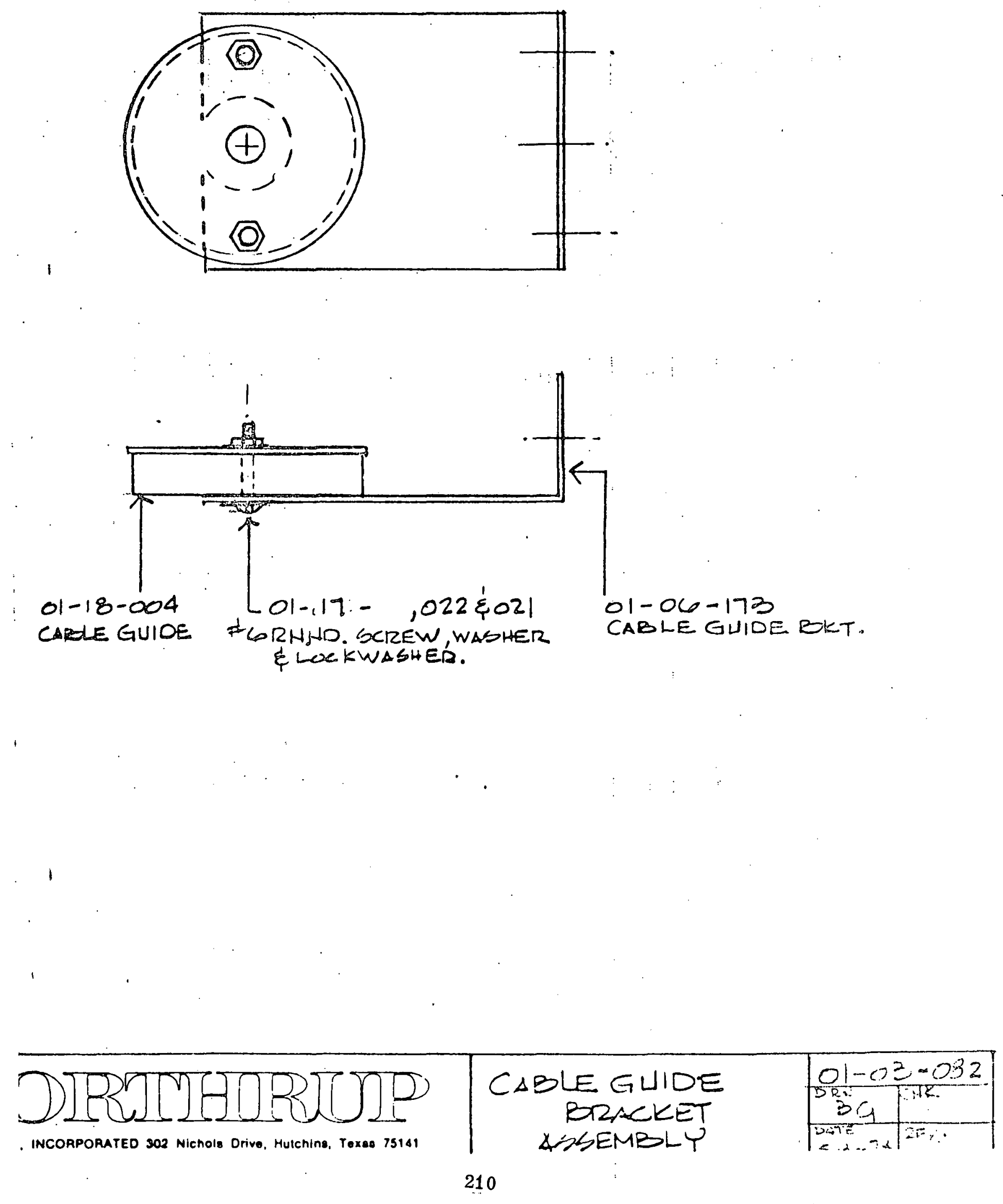


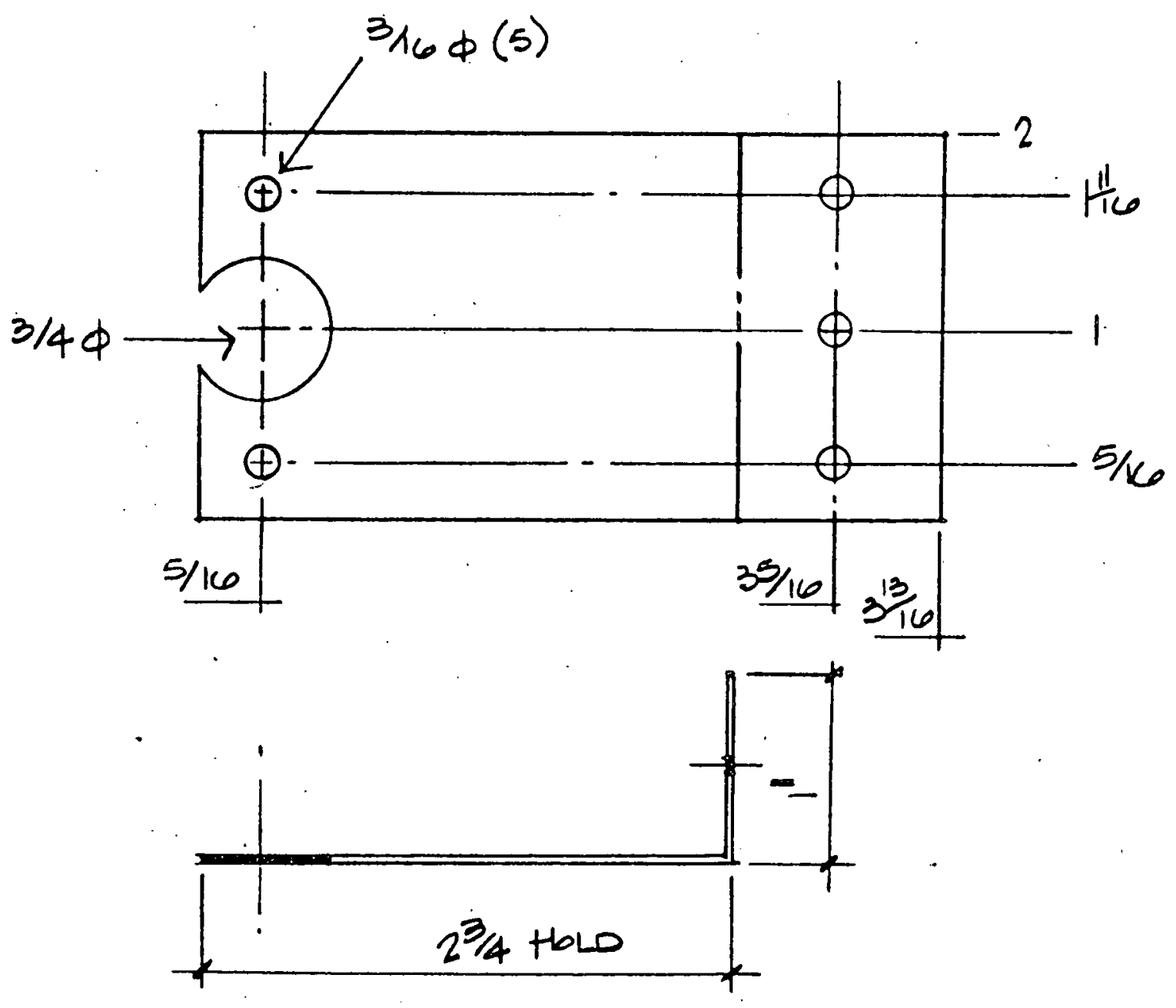

$$
\frac{\text { MATERIAL } 16 \text { GA GAL. (9'-OI) }}{\text { SHEAR SIZE } 2 \times 3^{13} \frac{1}{16} .}
$$

-

211 

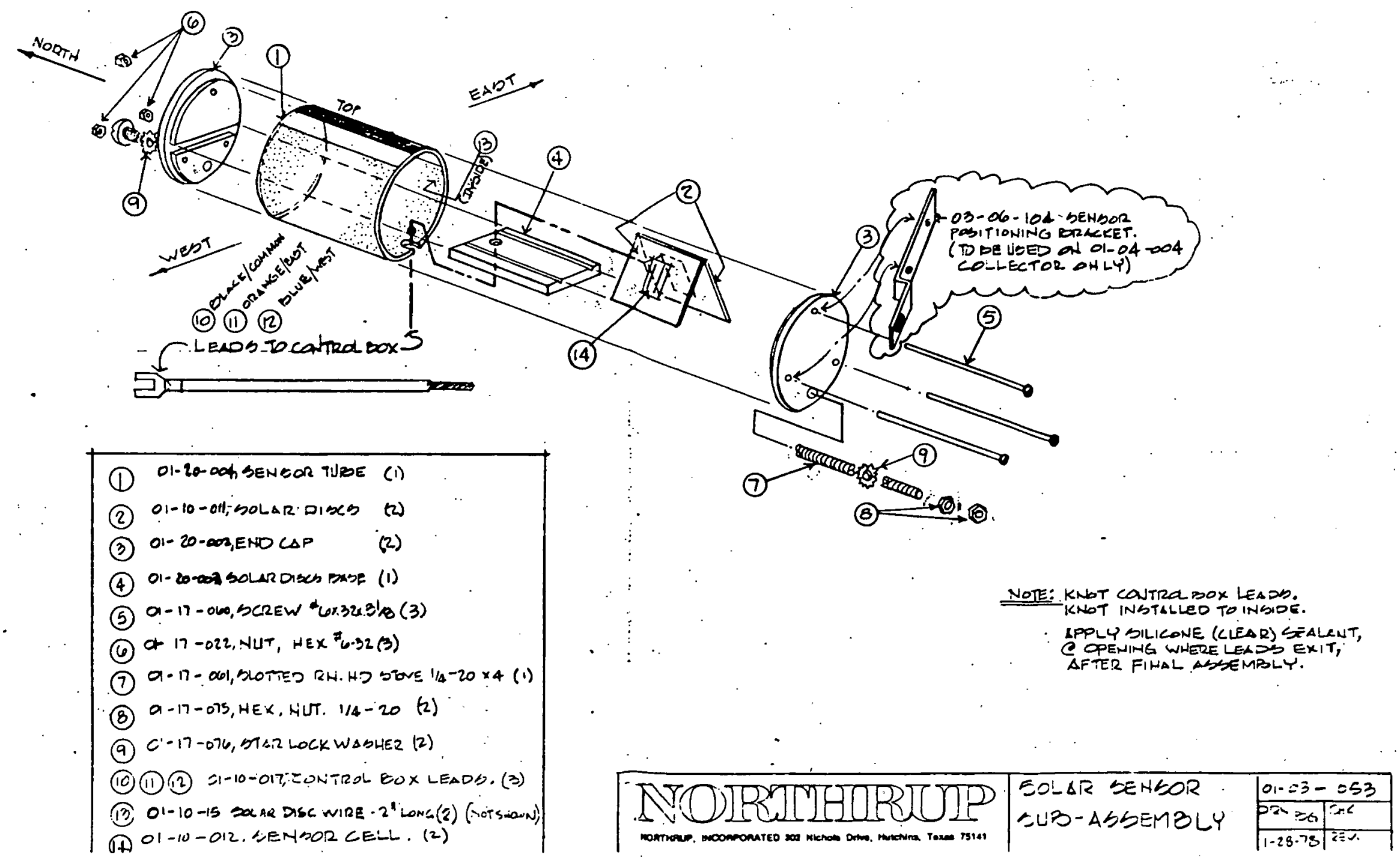


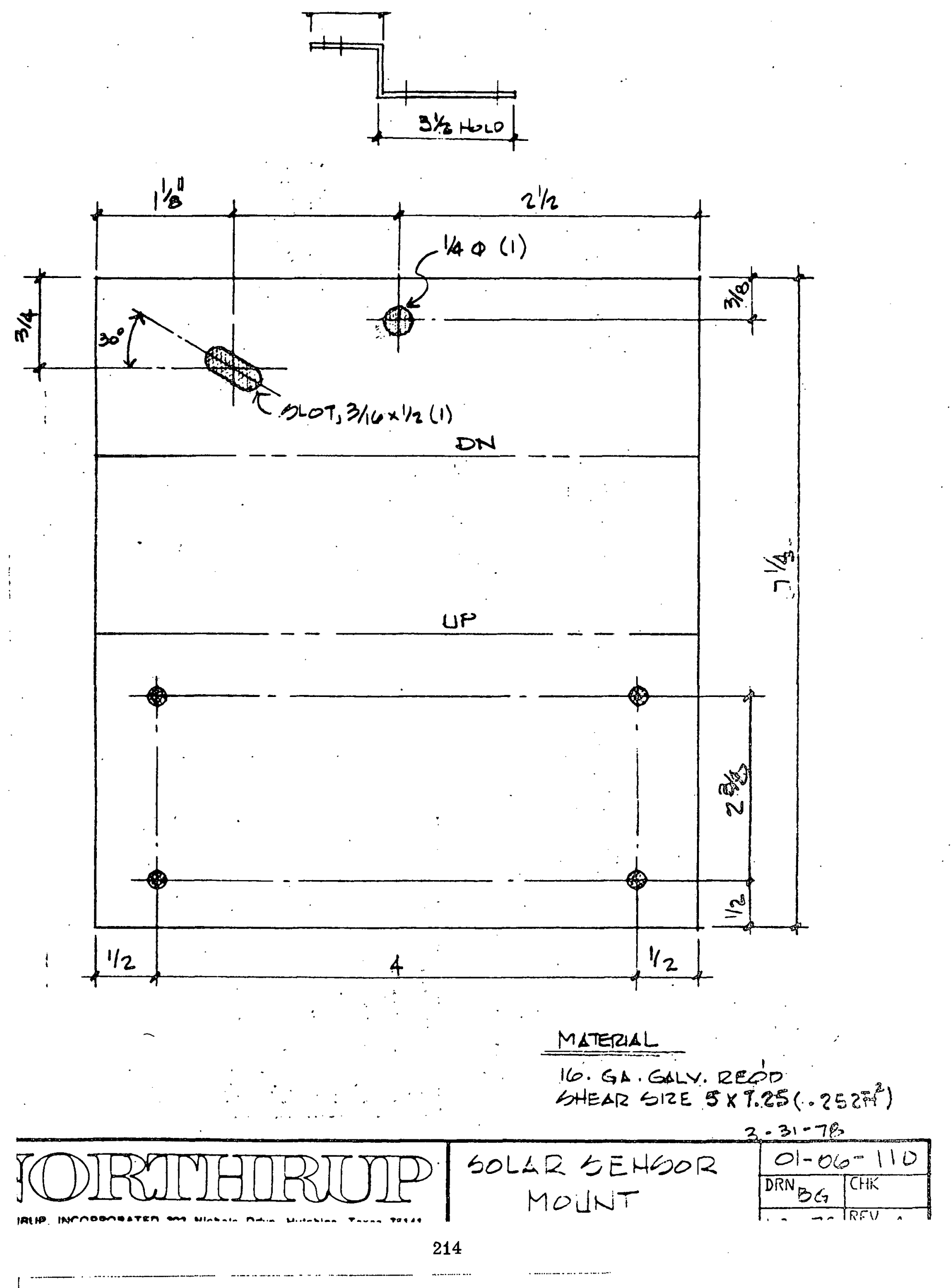



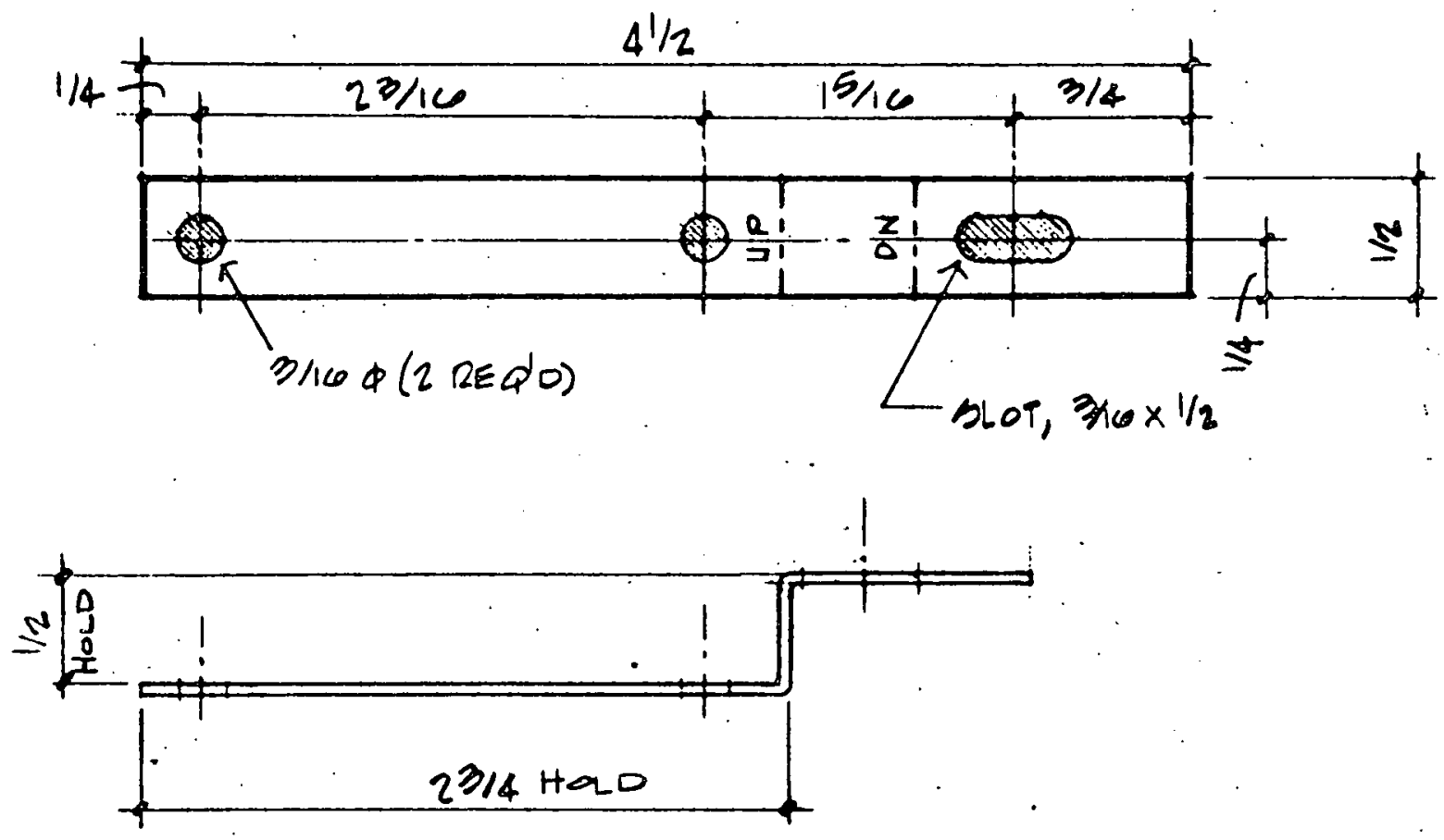

$\frac{\text { MATEZILL: }}{\text { 16 GA. OAW. REQP }(01-01-001)}$ DHEGR GRE $4^{\prime} \mathrm{H}^{\prime} \times \mathrm{D}^{\prime \prime} \mathrm{L}^{\prime \prime}$ (.015 SA.RT.) ALL DEND $90^{\circ}, \ldots .016$.

i.

NORTITHORUND MORTHRUP. INCORPORATEO 302 NICHOl. OHve. Mutchins. Texee 73141

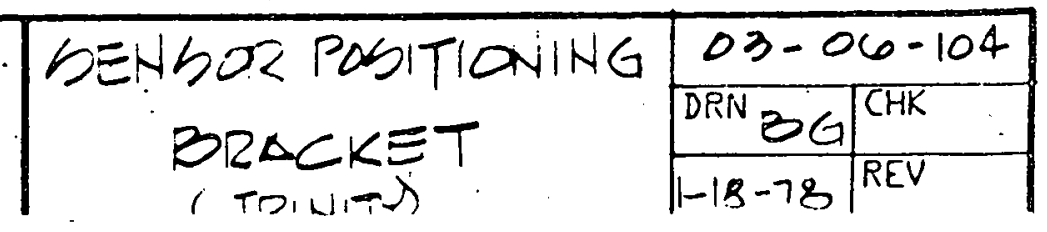




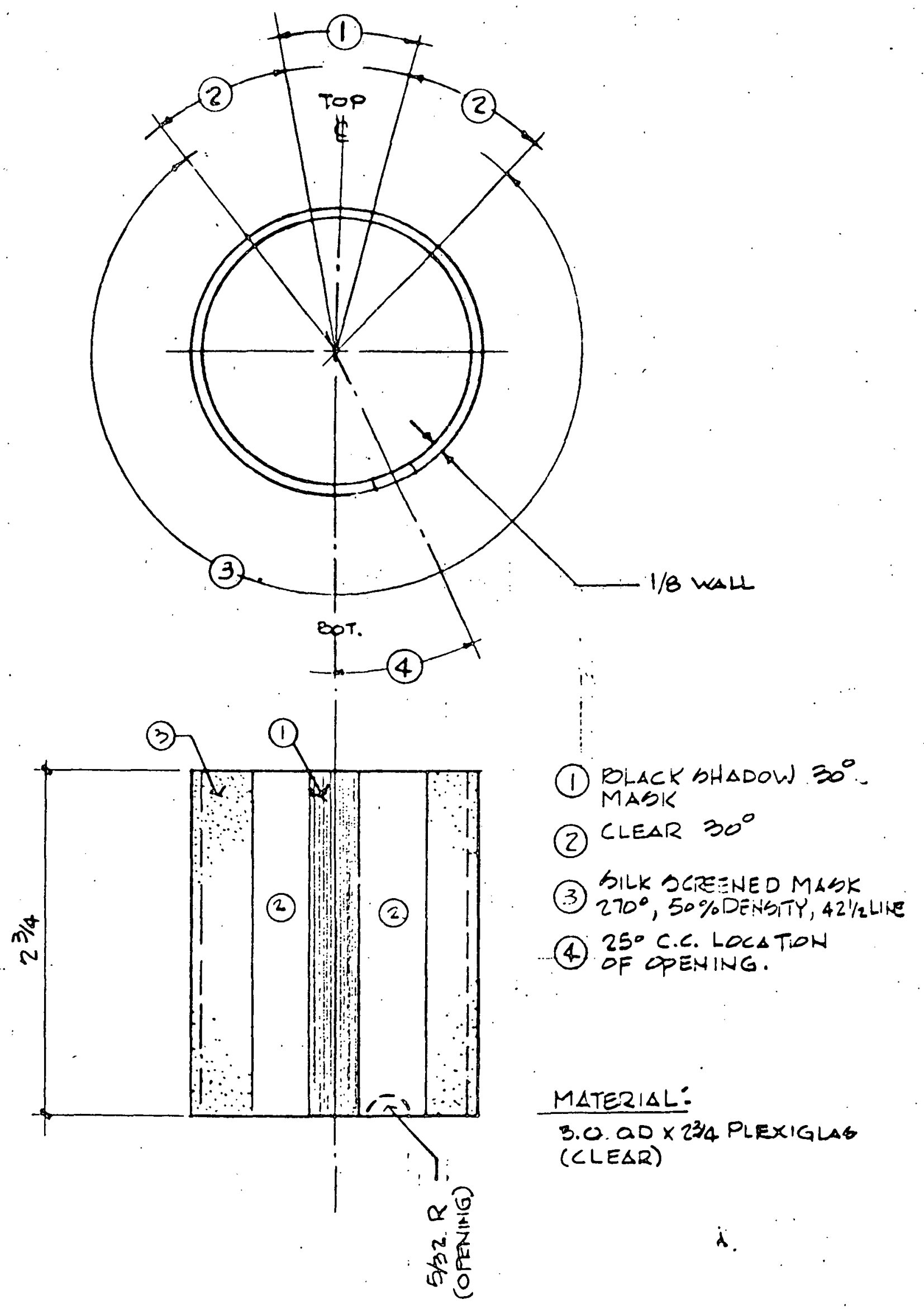

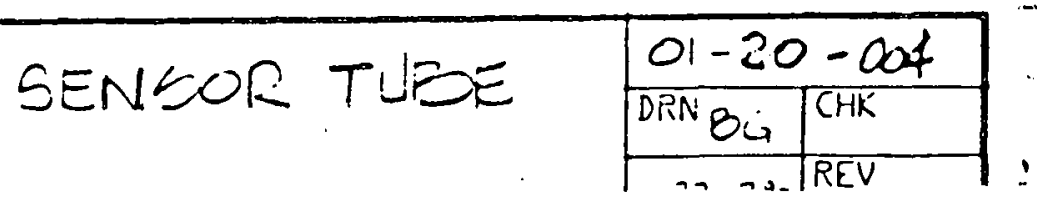




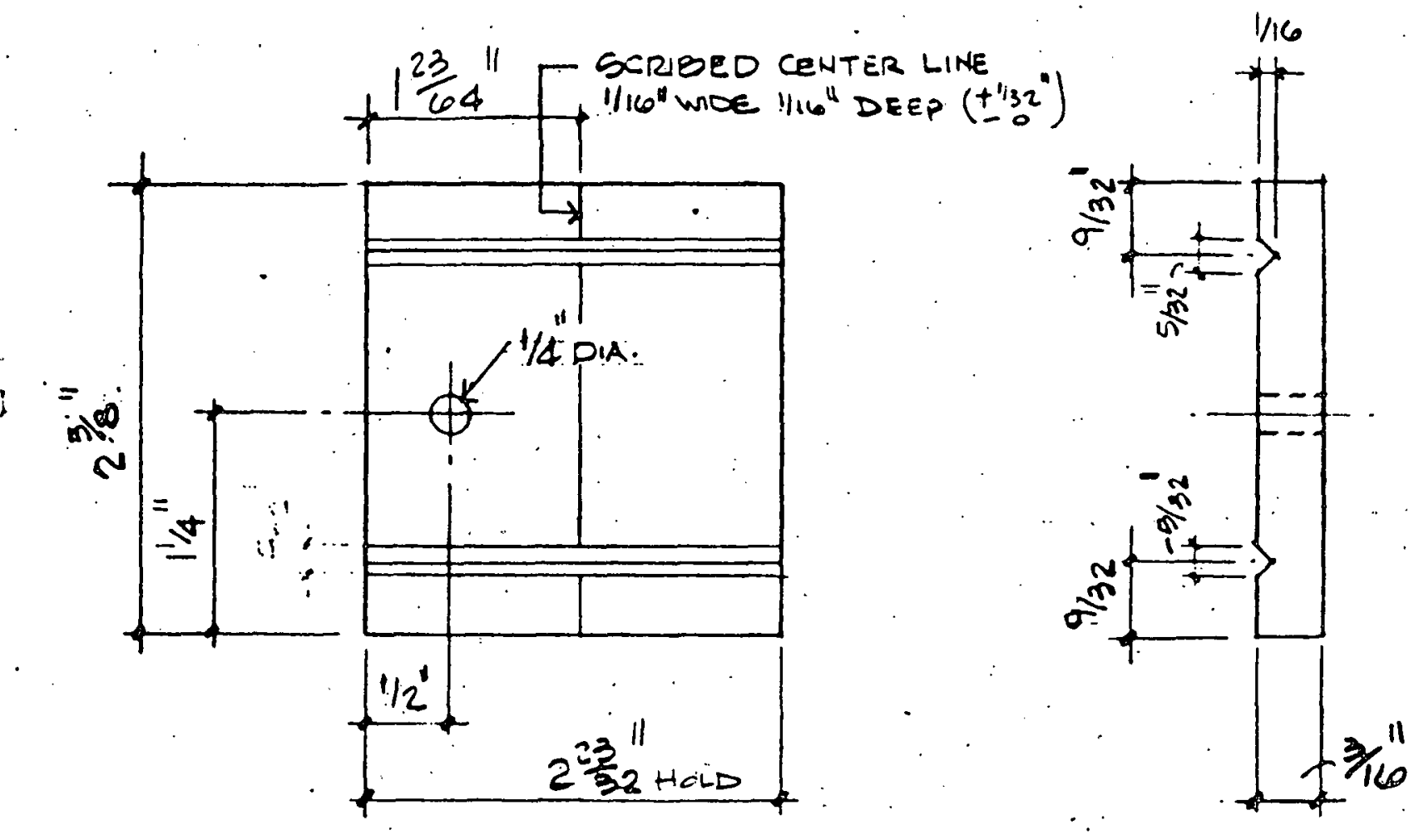

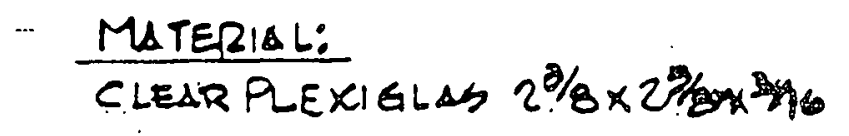

i.

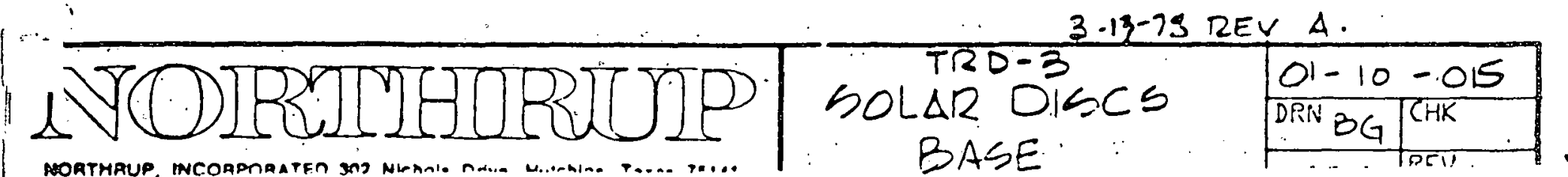




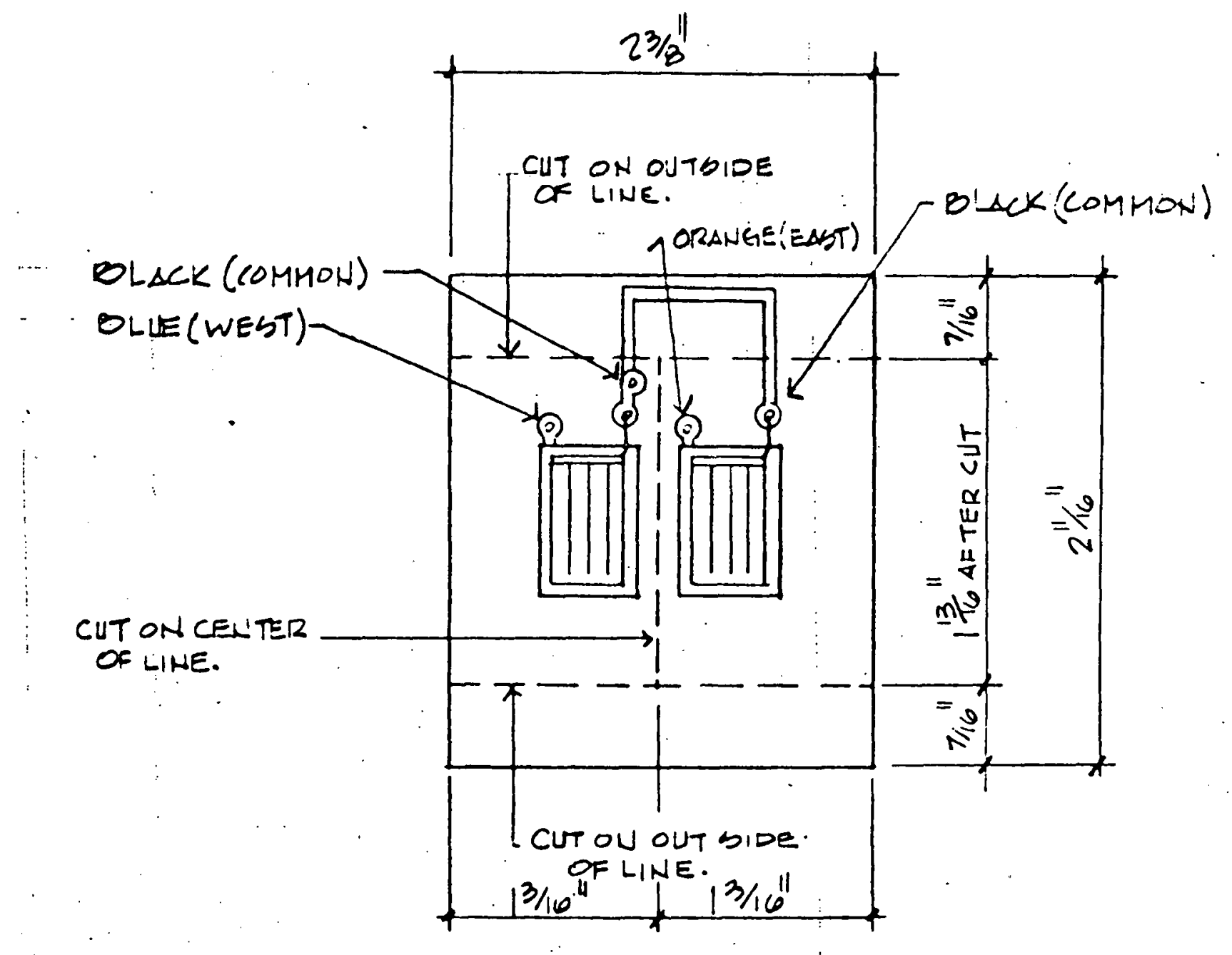

NOTE:

CUT ENDS OFF FIRST THEN CUT UNIT IN HSLF. 


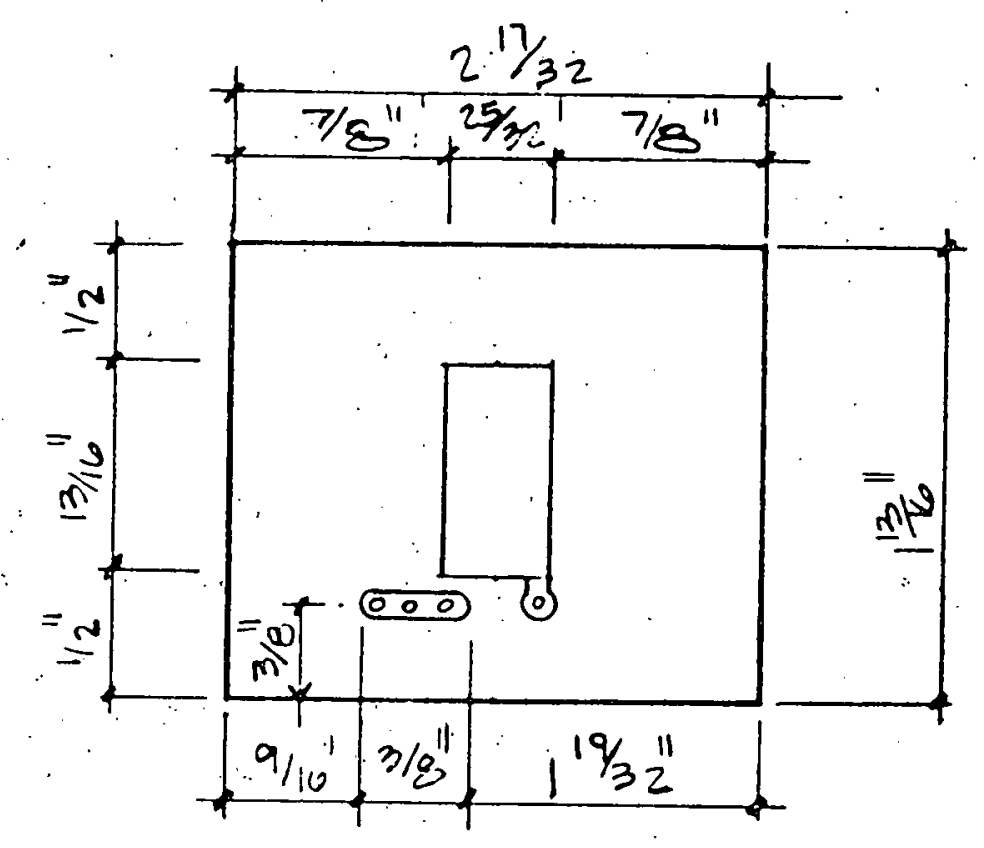

MATERLLE PC. POOAZO ANO CONOLTTOO.

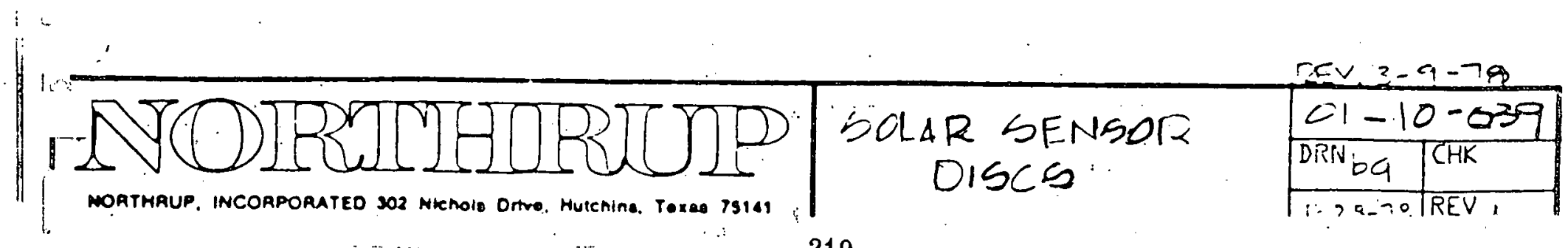


